University of Louisville

ThinkIR: The University of Louisville's Institutional Repository

$12-2018$

\title{
Innate immunity, the hepatic extracellular matrix, and liver injury: mathematical modeling of metastatic potential and tumor development in alcoholic liver disease.
}

Shanice V. Hudson

University of Louisville

Follow this and additional works at: https://ir.library.louisville.edu/etd

Part of the Biological and Chemical Physics Commons, Biostatistics Commons, Microarrays

Commons, Multivariate Analysis Commons, and the Translational Medical Research Commons

\section{Recommended Citation}

Hudson, Shanice V., "Innate immunity, the hepatic extracellular matrix, and liver injury: mathematical modeling of metastatic potential and tumor development in alcoholic liver disease." (2018). Electronic Theses and Dissertations. Paper 3075.

https://doi.org/10.18297/etd/3075

This Doctoral Dissertation is brought to you for free and open access by ThinkIR: The University of Louisville's Institutional Repository. It has been accepted for inclusion in Electronic Theses and Dissertations by an authorized administrator of ThinkIR: The University of Louisville's Institutional Repository. This title appears here courtesy of the author, who has retained all other copyrights. For more information, please contact thinkir@louisville.edu. 
INNATE IMMUNITY, THE HEPATIC EXTRACELLULAR MATRIX, AND LIVER INJURY: MATHEMATICAL MODELING OF METASTATIC POTENTIAL AND TUMOR DEVELOPMENT IN ALCOHOLIC LIVER DISEASE

By

Shanice V. Hudson

B.S. Massachusetts Institute of Technology, 2013

\author{
A Dissertation \\ Submitted to the Faculty of the \\ School of Interdisciplinary and Graduate Studies \\ of the University of Louisville \\ in Partial Fulfillment of the Requirements \\ for the Degree of \\ Doctor of Philosophy \\ School of Interdisciplinary and Graduate Studies \\ University of Louisville \\ Louisville, KY
}

December 2018 



\section{INNATE IMMUNITY, THE HEPATIC EXTRACELLULAR MATRIX, AND LIVER INJURY: MATHEMATICAL MODELING OF METASTATIC POTENTIAL AND TUMOR DEVELOPMENT IN ALCOHOLIC LIVER DISEASE}

By

Shanice V. Hudson

B.S. Massachusetts Institute of Technology, 2013

Dissertation Approved on

$11-27-2018$

By the following Committee:

Hermann B. Frieboes, Ph.D. (Mentor)

Gavin E. Arteel, Ph.D. (Co-Mentor)

Levi J. Beverly, Ph.D.

Michael L. Merchant, Ph.D.

Matthew C. Cave, M.D. 


\section{DEDICATION}

This dissertation is lovingly dedicated

to my framily,

to my family,

to Nate \& Jake,

in the hope that I can inspire achievement,

\& to my husband,

Garmell Hudson,

who has made a

lifelong commitment to dream chasing. 


\section{ACKNOWLEDGEMENTS}

To so many people. Here firstly, to Gavin and Jule Arteel, two of the brightest, warmest people I have ever worked with, for giving me a chance and a family. They worked so hard, and somehow also effortlessly, to help completely turnaround my graduate experience by providing an environment where I could learn and grow and thrive (Arteelian-Thurmanite forever!).

I would be remiss to not also thank Lacey McNally, for giving me the advice I needed, and my beginnings. To Drs. Paul DeMarco, Hermann Frieboes, Guy Brock, Levi Beverly, Michael Merchant, and Matt Cave for supporting me as directors of my program, and dissertation committee. To Dr. Leah Siskind for her friendship, support, and constancy in all things. To Drs. William Dean, Richard Miller, Chi Li, Shirish Barve, Swati Joshi-Barve, Irina Kirpich, and Martin O’Toole for their help and support.

To the faculty and staff of the Departments of Pharmacology and Toxicology, Bioengineering, and Bioinformatics and Biostatistics, for providing a robust academic environment and estimable faculty to foster my development as an independent scientist.

To Doug Saforo, I cannot in a million years thank you enough for everything. To Dennis and Jeffrey Warner, Shubha Gosh Dastidar, and Sabine Waigel, thank you for all your help with my project. 
Many thanks to all my lab mates, past and present: To Dr. Lauren Poole and Dr. Anna Lang for support and friendship, sanity checks and more laughs than should be had in a laboratory, kindness and concern. Thank you as well to Christine Dolin, Brenna Kaelin, Daniel Wilkey, Grace Mahlbacher, Hunter Miller, and Jamie Young, for their support, experimentally and otherwise.

To Aaron Neely for so much that was so needed. To my framily everywhere, this is all for you. You know what it is; you're irreplaceable. Without you all, what would any of it be worth? Here now in life and forever.

To my loves, Garmell, Nathan, and Jacob. I can only hope that l've made you feel any fraction of the pride I feel to have been chosen in this life to be the wife and mother of our humble clan. I'm living only for our days, our smiles, our plans, our successes. I pray we make it to our new dawn, all of us happy and in good health to enjoy it. Thank you for letting me steal so much of our time and disrupt the normalcy of our lives to fulfill my dreams. I owe each of you the same consideration and more-my eternal love.

To my parents and my family... Finally I get to begin to pay the debt forward, in your names, on the shoulders of titans. I'm forever grateful. 


\section{TABLE OF CONTENTS}

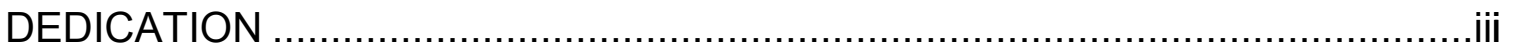

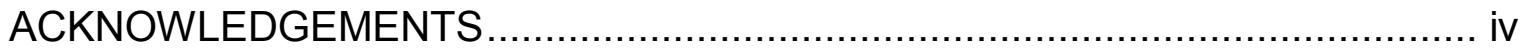

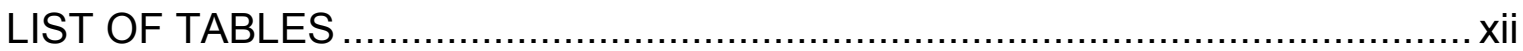

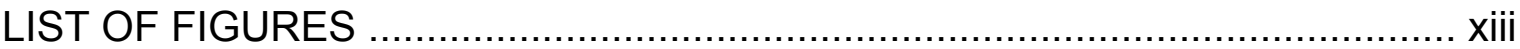

CHAPTER

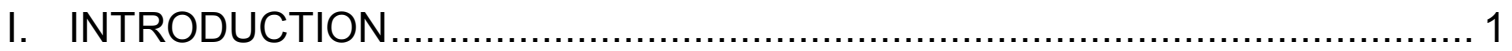

A. Background and rationale for the study ......................................... 1

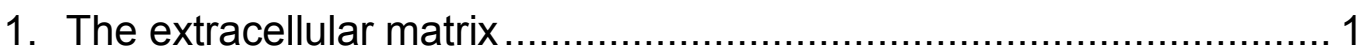

2. Integrins: ECM function and role in cancer.................................... 3

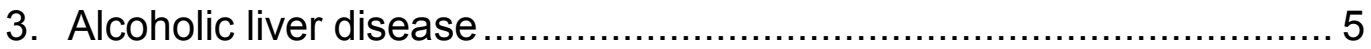

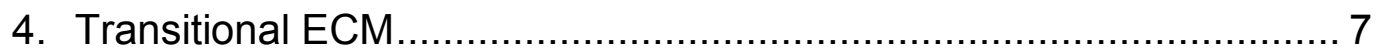

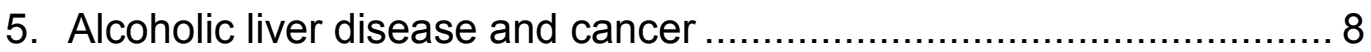

6. Macrophages and the cancer microenvironment .......................... 11

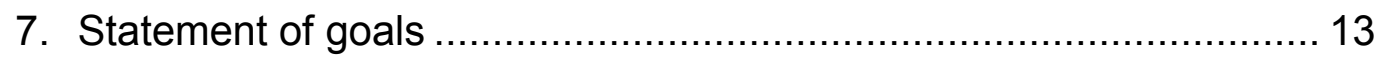

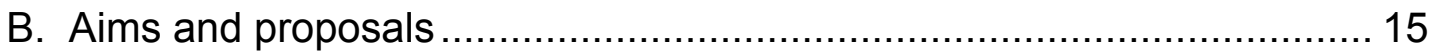

1. To computationally predict integrin binding kinetic profile based on experimental data analysis of integrin-mediated changes to the

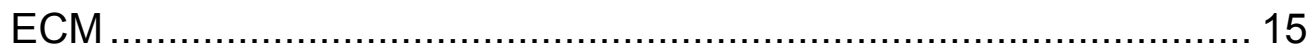

2. Characterizing modulation of macrophage polarization .................. 15

3. Evaluating immunomodulatory stimuli via a 2D tumor growth model of liver ECM and macrophages ................................................... 16

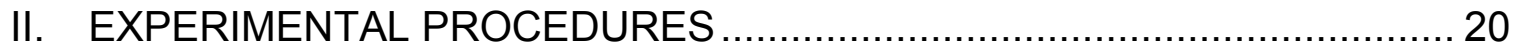




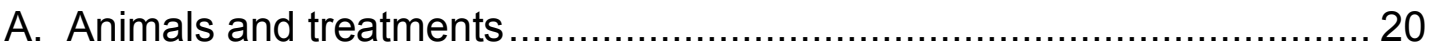

1. Animal sacrifice, tissue collection and storage ............................... 20

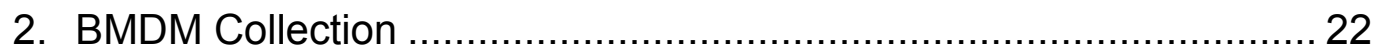

3. Lieber-DeCarli alcohol diet model ................................................ 22

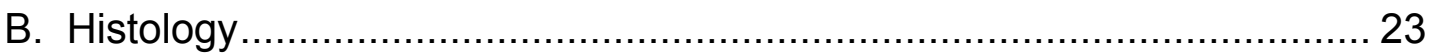

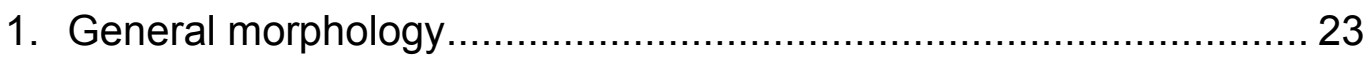

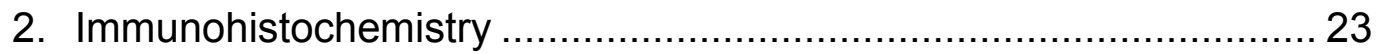

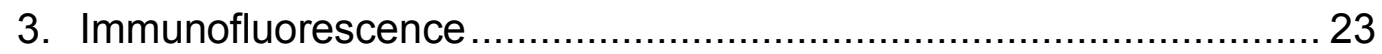

4. Visualization of green fluorescence in frozen tissue sections ...........23

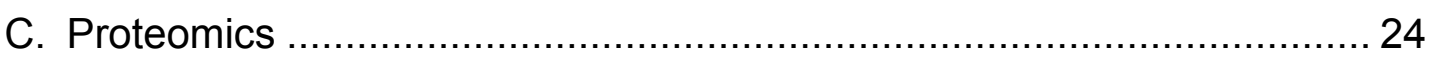

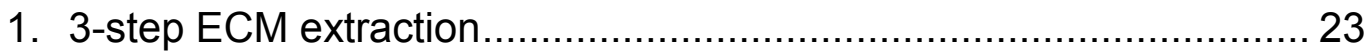

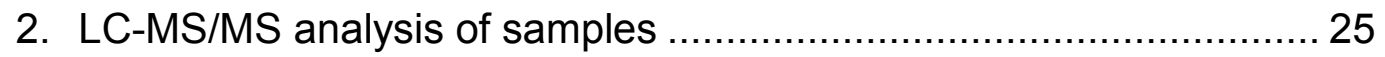

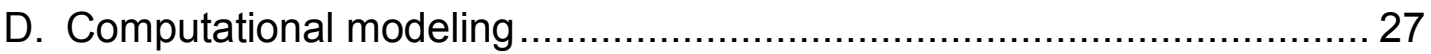

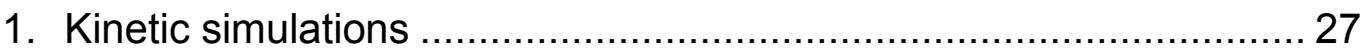

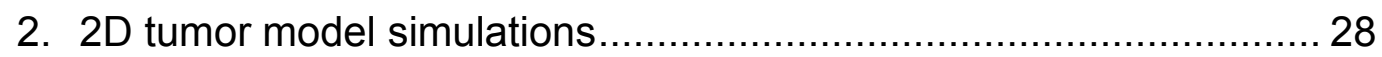

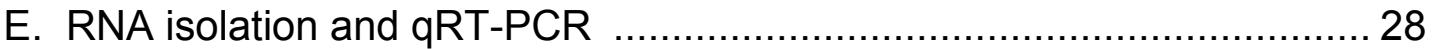

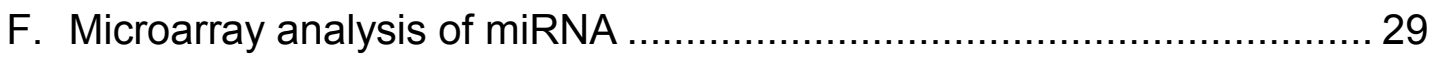

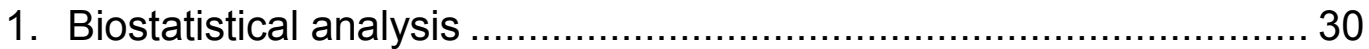

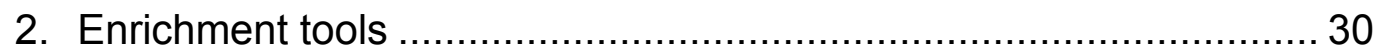

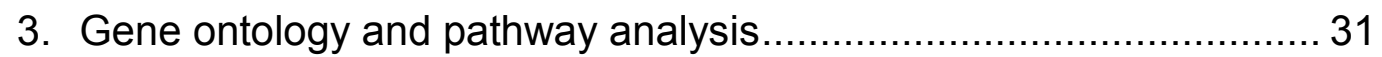

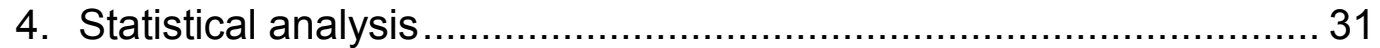

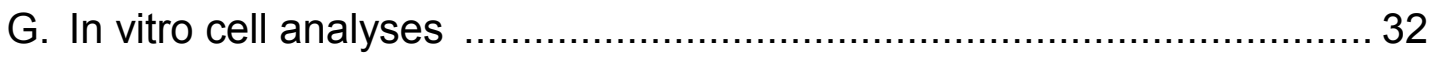

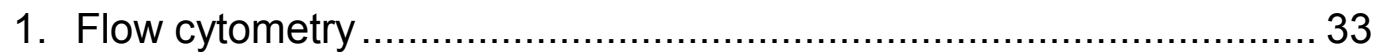




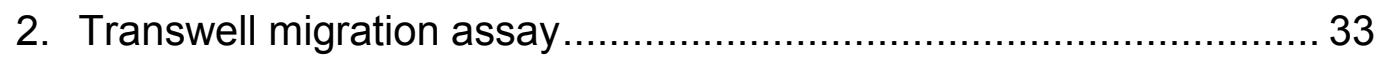

3. Tumor cell/macrophage indirect co-culture assay .......................... 34

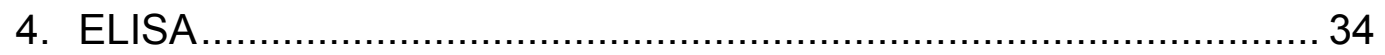

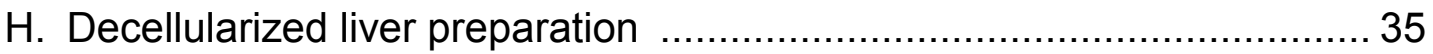

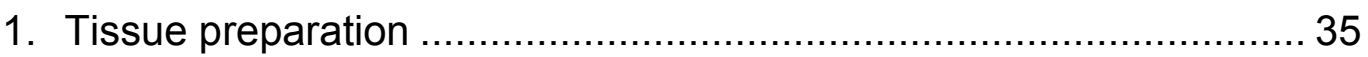

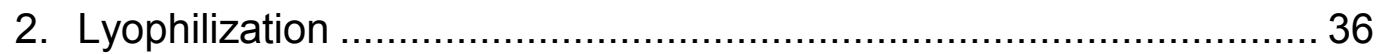

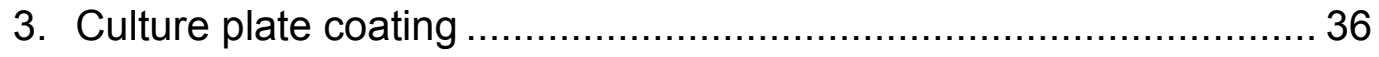

III. MODELING THE KINETICS OF INTEGRIN RECEPTOR BINDING TO HEPATIC EXTRACELLULAR MATRIX PROTEINS .................................. 38

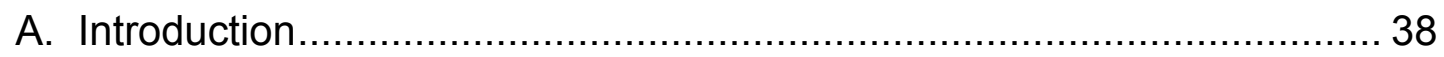

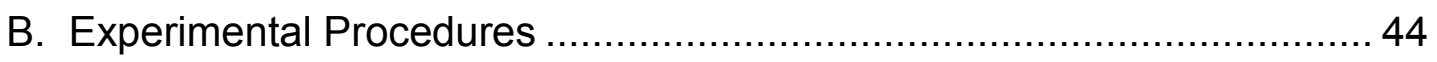

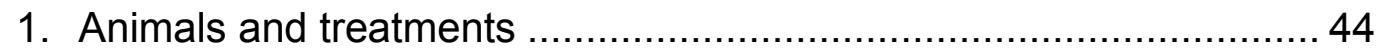

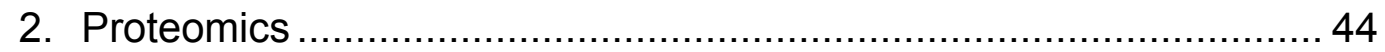

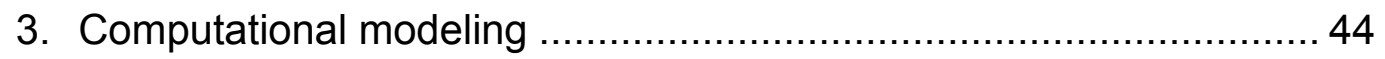

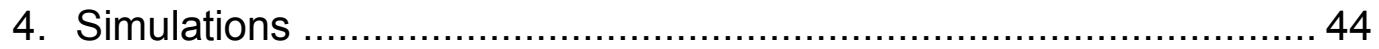

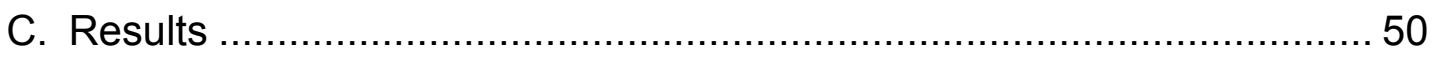

1. $\mathrm{CCl}_{4}$ exposure causes robust fibrotic liver scarring ….....................50

2. Differential protein expression of $\mathrm{CCl}_{4}$ exposed liver ECM ..............5 54

3. Qualitative changes to hepatic matrisome ..................................... 59

4. Kinetic simulations indicate increased affinity and avidity for ECM

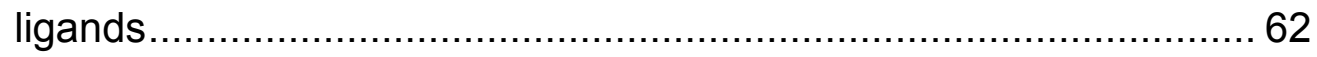

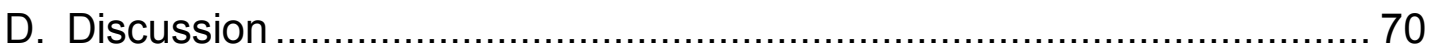


IV. ARSENIC ATTENTUATES LIPOPOLYSACCHARIDE-STIMULATED

POLARIZATION IN MURINE BONE MARROW DERIVED

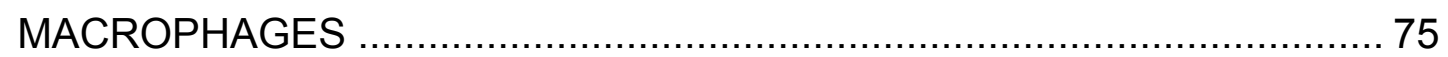

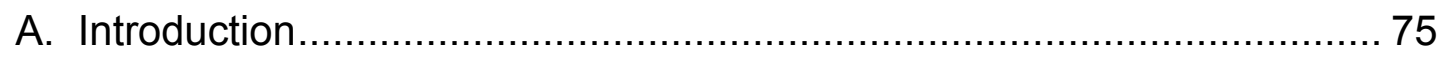

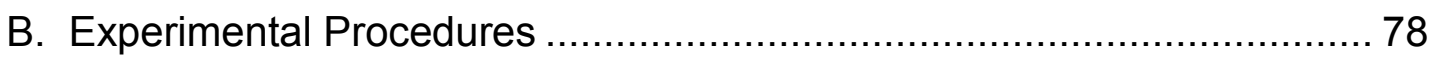

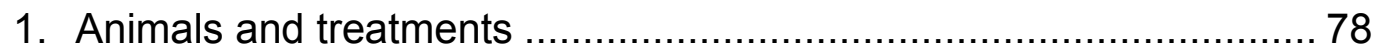

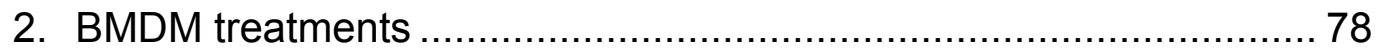

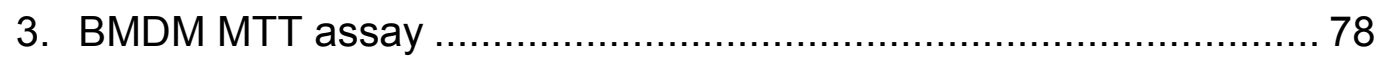

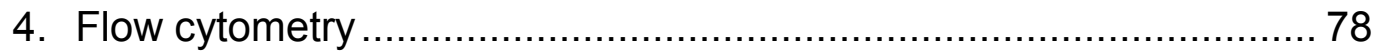

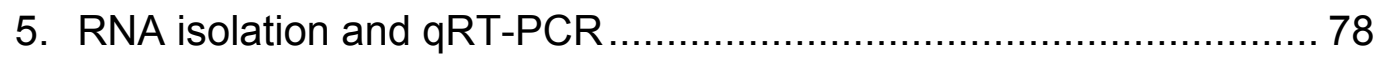

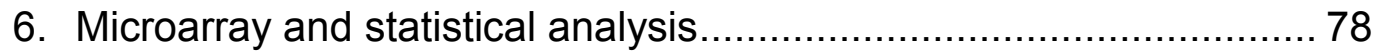

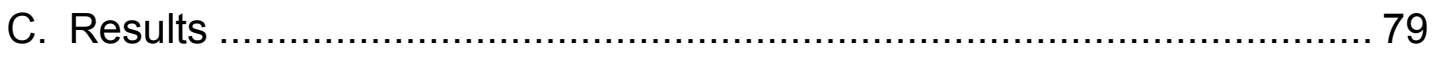

1. Sodium arsenite attenuates LPS-induced polarization of BMDMs, but

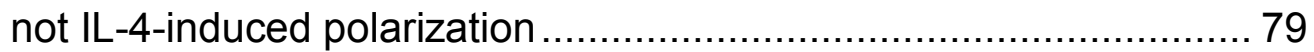

2. Sodium arsenite modifies constitutive and LPS-induced mRNA expression levels of TNF- $\alpha, \mathrm{IL}-1 \beta$, iNOS, and PAI-1 ....................... 85

3. Differential miRNA expression profiles of BMDMs treated with As(III),

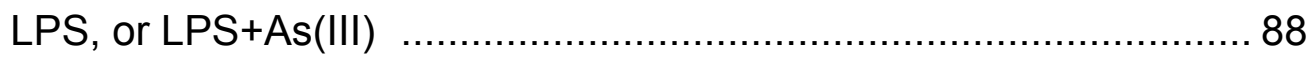

4. Differential miRNA expression of miR-301b-validation experiments . 94

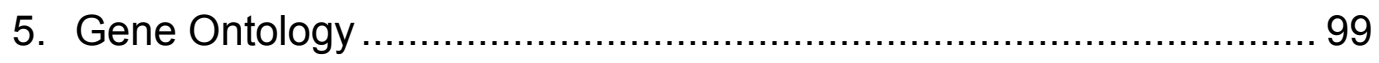

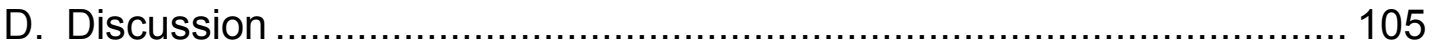

V. A COMPUTATIONAL 2D TUMOR MODEL-MACROPHAGES AND ECM 111

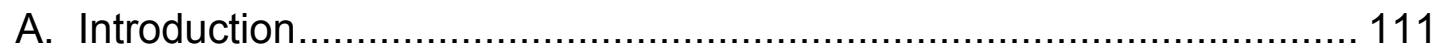


B. Experimental Procedures

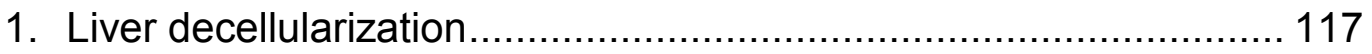

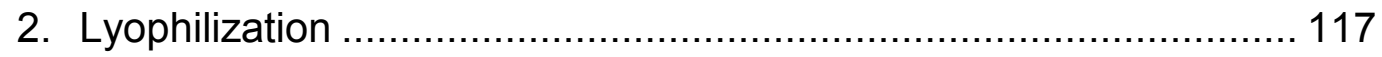

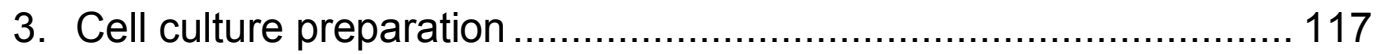

4. Macrophage and tumor indirect co-culture assay ......................... 117

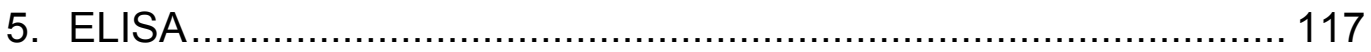

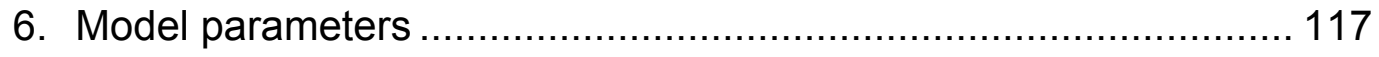

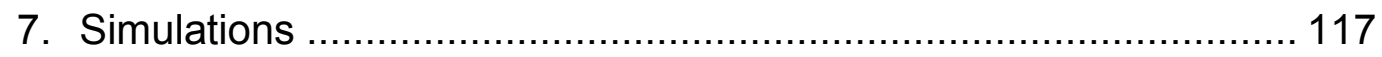

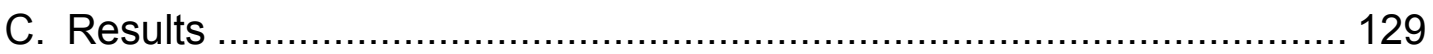

1. Validation of liver decellularization ............................................ 129

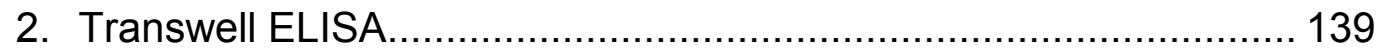

3. 2D visualization of tumor growth in microenvironment .................. 142

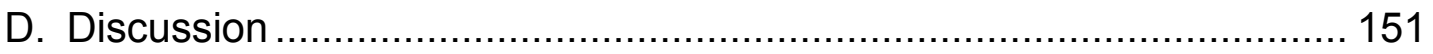

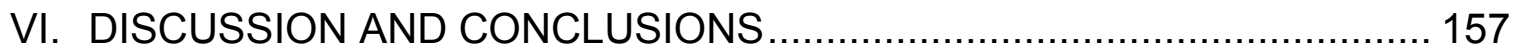

A. Restatement of goals and questions …......................................... 157

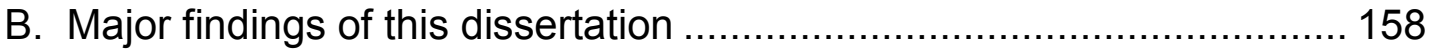

1. Altered hepatic ECM protein composition resulting from ECM remodeling associated with liver injury changes binding profile for cognate integrin receptors.

2. Arsenic attenuates lipopolysaccharide-stimulated polarization in murine bone marrow derived macrophages 
3. Immunomodulatory stimuli and the density of extracellular matrix in the tumor microenvironment affects in silico tumor growth in a spatiotemporal mathematical model of tumor growth ............................ 163

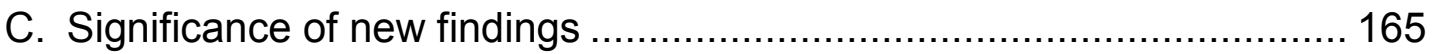

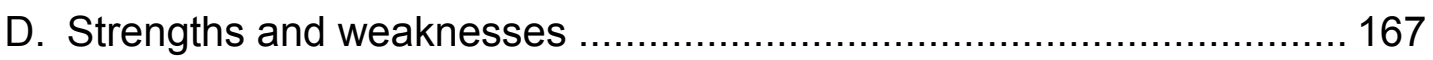

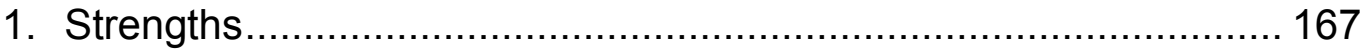

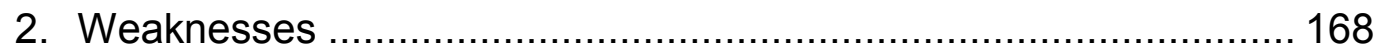

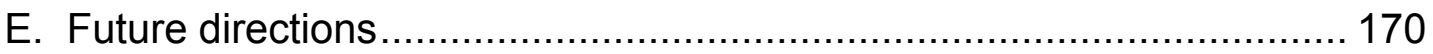

1. Could a stochastic model of integrin binding predict binding

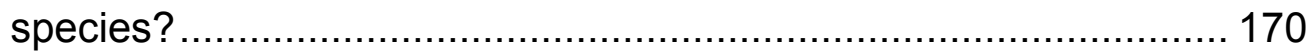

2. What is the mechanism by which miR-301b modulates macrophage polarization attenuation in LPS+Arsenic-treated BMDMs?.

3. Can translational value of computational tumor growth model be enhanced by in vivo model data? ............................................... 172

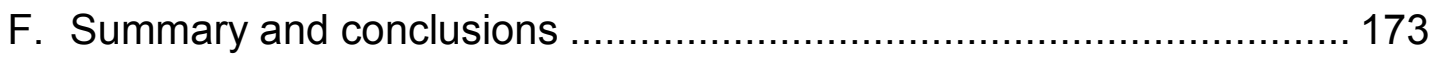

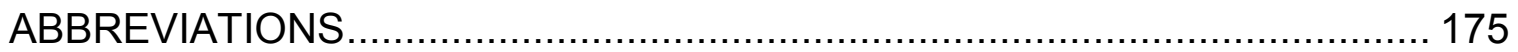

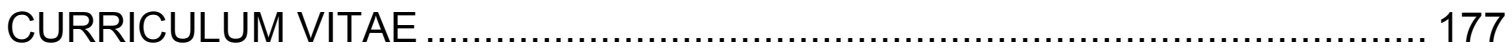

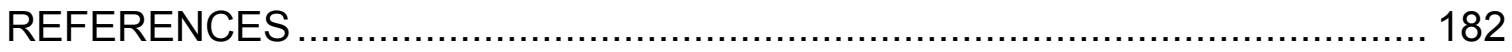




\section{LIST OF TABLES}

\section{TABLES}

Table 3.1: Quantitative differential protein expression of liver ECM following 4

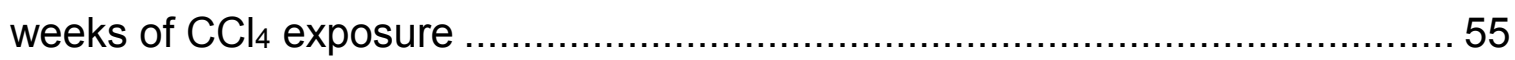

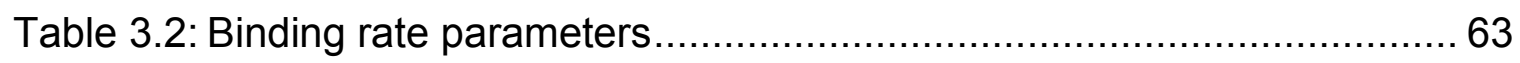

Table 3.3: Equilibrium binding constants for receptor aggregation model .......... 64

Table 3.4: Steady-state values for simulations of binding ................................ 67

Table 4.1: Listing of differentially expressed miRNAs................................... 92

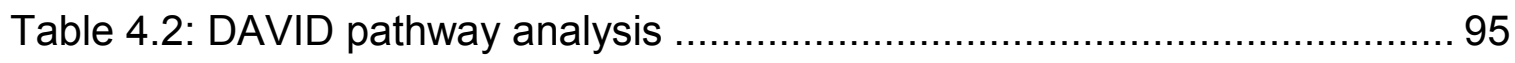

Table 5.1: Macrophage-associated cytokines ……...................................... 119

Table 5.2: Tumor parameters and values ................................................... 119

Table 5.3: Macrophage model associated parameters .................................. 123

Table 5.4: Macrophage model simulation conditions .................................... 125

Table 5.5: Macrophage cytokine rate parameters ….................................... 129 


\section{LIST OF FIGURES}

\section{FIGURES}

Scheme 3.1: Repertoire of alpha and beta integrin subtype dimerization

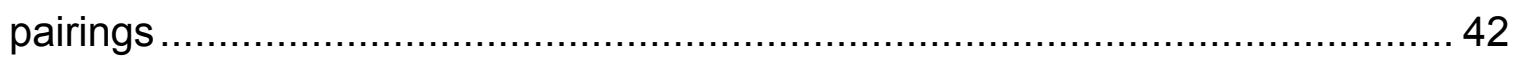

Scheme 3.2: Kinetic model description ...................................................... 46

Scheme 3.3: Schematic of aberrant ECM accumulation following $\mathrm{CCl}_{4}$ injury.... 51

Figure 3.1: Qualitative Venn diagram of proteomic data................................ 57

Figure 3.2: Immunofluorescent staining of hepatic cryosections ….................... 60

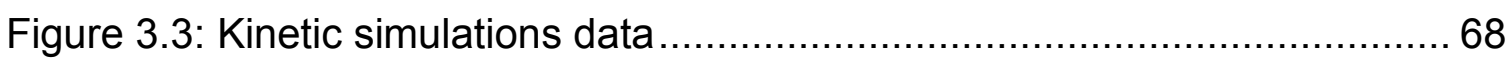

Figure 4.1: Dose optimization for LPS and sodium arsenite to find lowest

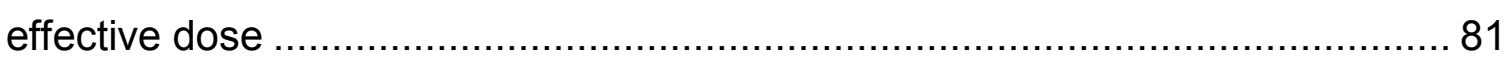

Figure 4.2: Flow cytometric analysis of macrophage polarization..................... 83

Figure 4.3: Effect of sodium arsenite on constitutive and LPS-induced mRNA

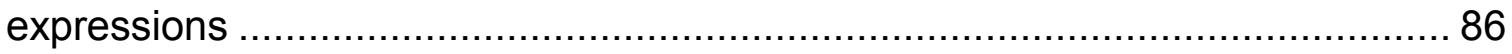

Figure 4.4: MicroRNA (miRNA) transcriptome analysis ................................... 90

Figure 4.5: Validation of miRNA expression profiles of treatment groups.......... 97

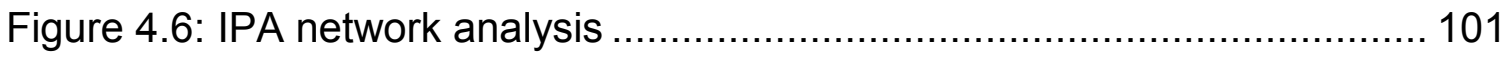

Figure 4.7: IPA Diseases and Biofunctional analysis................................... 103

Scheme 5.1: Natural history of liver disease ……........................................ 115

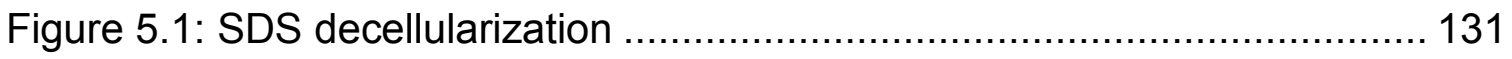

Scheme 5.2: Tumor transwell migration assay ............................................ 133 
Figure 5.2: Tumor transwell migration cell counts

Scheme 5.3: Tumor/macrophage indirect co-culture assay.

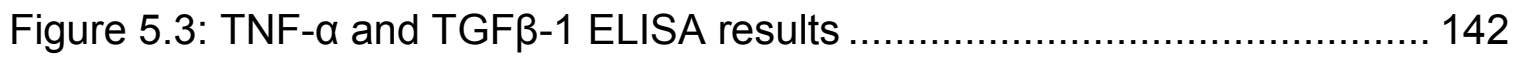

Figure 5.4: 2D ECM-dependent simulations (monocyte populations only) ....... 143

Figure 5.5: 2D ECM-macrophage simulations …........................................ 145

Figure 5.6: Simulation analyses of tumor radii and vessel surface area........... 147

Figure 5.7: Macrophage cell counts and population fractions ......................... 149 


\section{CHAPTER I}

\section{INTRODUCTION}

\section{A. Background and rationale for this study}

\section{The Extracellular Matrix}

The extracellular matrix (ECM) functions as a bioactive scaffold, providing tissue architecture, strength, and elasticity. Although the ECM is historically characterized in relation to its structural components, the extracellular matrix is now known to plays far more roles; it is critical to cell adhesion, proliferation, motility, and even innate immunity. The ECM is a dynamic hub for morphogenesis, cell signaling, immune cell recruitment, and many other fundamental processes [1]. The complex network of collagens that comprise the basement membrane lend structure and tensile strength, while proteoglycans and glycoproteins provide the interstitial hydrating matrix and compartment for growth factor storage. The essential function of the ECM cannot be understated; indeed, genetic mutations in matrix components result in a spectrum of connective tissue pathologies [2-4], if not embryonic lethality [5, 6], which underlines its inherent function in all tissues. Dysregulation of ECM components is the etiologic basis of various pathologies, especially fibrosis and invasive neoplasia. Therefore, balanced regulation of ECM production and degradation is necessary for maintenance of organ structure and function. 
Extracellular matrix remodeling has a critical role in tissue homeostasis and repair $[7,8]$. In contrast, uncontrolled or aberrant remodeling in response to stress contributes to angiogenesis, inflammation, fibrotic diseases, as well as tumor invasion and metastasis in cancer [7, 9]. The ECM mediates cell biochemical signaling to regulate functions of tissues and movement of cells within the interstitium, as well as absorbing physical signals from the environment that affect cell organization and form. Sensing and sensitivity are key to this elasticity, and tactile density and pressure dictate actions cells correspondingly take towards migration, differentiation, and adhesion. A broad classification of proteins associated with the extracellular matrix has been termed matrisome; this incorporates the ECM proteome, etc. In Naba et al. [10], ECM glycoproteins, fibrillar proteins, collagens, and proteoglycans are designated to the "core matrisome." Matrisome-associated proteins comprise the more dynamic set of ECM targets that regulate the ECM; enzymes, secreted factors, and ECM proteins $[10,11]$.

Liver ECM is responsive to microenvironment, integrating cell signals to coordinate diverse pathways of action; its interaction with parenchymal function is critical in maintaining tissue integrity and non-pathogenic matrix remodeling for tissue repair [12]. The ECM of many other organ system tissues share similar functions, though among them, the liver has the greatest capacity for regeneration of any visceral organ [13]. Consequently, it can manage acute insults to restore tissue homeostasis via degradation of the extracellular matrix and then tissue replacement via matrix deposition. However, dyshomeostasis of 
matrix metabolism is a key feature of almost every form of chronic liver disease, regardless of etiology; e.g., conditions that result from either direct or secondary liver insult, whether from alcohol exposure, metabolic disease, or exogenous toxicants. Thereby, the ECM can be altered via inflammatory cascades that trigger progressively irreversible remodeling of the ECM.

2. Integrins: ECM function and role in cancer

Integrins are transmembrane receptors that facilitate interaction between cells and the extracellular matrix, as well as cell-cell interactions. Cell-surface integrin expression and activation mediates adhesion to ECM and leads to signal transduction that regulates proliferation, apoptosis, and differentiation, among other important processes [14-16]. Integrin receptor interactions are key mediators of cell attachment, migration, proliferation, among many other molecular processes, primarily via binding to extracellular matrix (ECM) glycoproteins, leading to bidirectional signal transduction that influences critical cellular functions. The composition of the ECM and the expression of their cognate receptors dictates cell behavior and dynamics. Vital to the maintenance of homeostatic conditions, integrins have become important therapeutic targets for diseases of dysregulation-namely various cancers, immune dysfunction, and chronic inflammation. Promiscuity among the repertoire of ECM ligands and integrin receptors, particularly those with Arg-Gly-Asp (RGD) binding motifs, means binding affinities vary for each combination. Understanding the kinetics of 
integrin binding can provide a profile of integrin interaction relative to the ECM proteomic signature.

Cell migration and cell-cell interactions that are modulated by integrins contribute to tumor invasion and metastasis $[17,18]$. Integrin-ECM interactions play critical roles in the processes of tumor cell arrest in the vasculature, extravasation towards secondary site, and invasion and colonization of micrometastases. Gulubova et al. [19] have shown in clinical samples of hepatic metastases from human colorectal and gastric cancer that ECM components such as tenascin-C, fibronectin, and their respective integrin receptors were found to often form capsules around liver metastases, indicating a role in facilitating invasiveness. Excessive ECM deposition occurs frequently in tumors evaluated to have poor prognosis [9], and the ECM phenotype can shift as tumors progress, resulting in changes to differential expression of integrins that occur in response to the microenvironment. For example, in pancreatic cancer, increased ECM protein deposition is classified as desmoplasia and is an important clinicopathologic biomarker disease progression and potential for chemorefractory response to therapeutic intervention [20,21]. In breast cancer, ECM proteins common to both mammary gland involution involved in normal remodeling of breast adipose tissue following parturition, and also to breast cancer, are similarly upregulated, indicating potential for breast tumors to utilize normal ECM turnover processes to facilitate their own growth [22, 23].

Dysregulation of integrin signaling in various cancers affect cancer cell processes of adhesion and invasion due to the interactions of tumor cells and the 
ECM in the context of the local microenvironment [24-26]. Upregulated integrin expression in tumor cells has been investigated and various novel targets have been identified for development of anti-cancer therapeutics. Tumor cell overexpression of $\alpha \mathrm{v} \beta 3$ integrin and its promiscuity in ligand binding influences the site of metastasis by facilitating tumor arrest in vasculature and invasion of tissues [27]. In recent studies, integrin subunit $\alpha 2$ was determined to mediate selective metastasis to the liver [28]. Dysregulations in ECM composition can provide a favorable microenvironment for metastatic seeding, contributing to desmoplastic stroma characteristic of excessive ECM deposition [29], and also creating a 3D scaffold that can influence the behavior of tumor cells and their integrin expression phenotype, directly influencing their sensitivity to therapeutics [30]. Mueller et al. found that transformed hepatic stellate cells (HSCs) contribute to stroma formation in liver metastases [31], and early fibrotic pathologies cause inflammation that leads to HSC transformation to myofibroblasts that increase ECM deposition, so there are clear links between the two phenomena.

\section{Alcoholic liver disease}

Alcohol use disorder and the health issues that arise from chronic alcohol abuse remain a significant healthcare issue worldwide, responsible for $5.9 \%$ deaths and $5.1 \%$ disability-adjusted life-years globally [32, 33]. Alcohol use is ubiquitous to human cultures, and its abuse often results in self-harm, accidents, and premature deaths [34, 35]. As a financial burden, alcohol abuse-related healthcare costs and loss of productivity have an impact of over 200 billion dollars annually in the US alone [36]. Besides lifestyle modification, currently 
there is no medical treatment for alcohol use disorders, and high rates of relapse among alcoholics following rehabilitation make it clear that research on related pathologies remains important for helping to manage the affected populations [37].

In addition to behavioral risks of alcohol abuse, chronic alcohol use is also a major health risk. The alcohol consumption pattern and length of drinking, including typical dose and frequency, are determining factors in risk assessment. Alcohol use disorders affect several primary target organs including the brain, heart, pancreas, kidneys, spleen, liver and lungs. Alcohol misuse is associated with long-term health risks, such as high blood pressure, stroke, pancreatitis, depression, dementia, and sexual dysfunction. Alcoholic liver disease (ALD), a spectrum of pathologies that progress from acute steatosis to fibrosis and cirrhosis, leads to damage to multiple organs and contributes to related comorbidities. In particular, advanced liver disease (i.e., cirrhosis) is the main cause of hepatocellular carcinoma ( $\mathrm{HCC})$ and end-stage liver failure via viral and alcoholic cirrhosis $[38,39]$.

Causes of liver disease include alcohol or toxicant exposure, or obesity. Regardless of the etiology of liver injury, the mechanisms of pathology similarly include secretion of inflammatory mediators by hepatocytes and Kupffer cells, resulting in activation of quiescent hepatic stellate cells (HSCs), upon which they transdifferentiate to myofibroblasts. Activated HSCs control secretion of fibrillar matrix proteins and collagens, as well as regulate expression of matrix metalloproteases and tissue metalloprotease inhibitors; these cells thereby 
coordinate ECM degradation and production to manage fibrogenic and proliferative signals to effect tissue repair and regeneration. In this activated state, homeostasis is maintained by resolution of liver injury and apoptosis of myofibroblasts following fibrogenesis [40]. Progression from normal liver homeostasis to transient fatty liver, or steatosis, occurs in response to short term exposure and is reversible, and is characterized by presence of large sharp fat droplets in hepatocytes. As insult and injury persists, steatohepatitis develops with fatty liver progressing to development of parenchymal inflammation and hepatocellular damage. If inflammation is prolonged, fibrosis can occur with accumulation of fibrillar collagens deposited to the ECM [41]. Fibrosis/cirrhosis characterized by aberrant fibrin deposition with bridging fibrosis; this heralds the clinical end stage, with limited treatment options besides organ transplantation [42].

\section{Transitional ECM}

A major focus of the Arteel group is to examine how alcohol activates transitional tissue remodeling in liver and the consequences of these events [43, 44]. Our group recently utilized proteomic data analysis [45] to demonstrate the dynamic response of the murine hepatic extracellular matrix (ECM) proteome (i.e., 'mastrisome') to alcohol exposure. Importantly, several of the ECM proteins that were either quantitatively or qualitatively changed by alcohol exposure are ligands for integrin receptor binding. Integrin receptors mediate attachment between a cell and surrounding tissues, which may be ECM or other cells [46]. 
Integrins transfer information from the ECM to the cell, allowing rapid and flexible responses to changes in the environment. Consequently integrins influence a myriad of molecular processes, including proliferation, angiogenesis, inflammation and apoptosis. As such, altered/aberrant ECM-integrin interactions can phenotypically change basal and induced response of the liver. Although this principle is partially understood in the context of hepatic fibrosis, little is known about the impact of these changes on earlier stages of liver disease.

5. Alcoholic liver disease and liver cancer In addition to primary injuries (e.g., alcoholic liver disease; see above), alcohol use also contributes to a broad range of secondary pathologies, including notably an increased risk of oncogenesis in several organs. Several studies having established links between alcohol consumption and cancers of the alimentary tract, as well as the breast, lung, and pancreas [47, 48]. The fibrotic pathology associated with chronic ALD leads to enhanced inflammation and higher risk in certain cancers for increased aggressiveness [49-53], and the associated induced dynamic tissue remodeling leads to desmoplasia and favorable conditions for tumor stromal overgrowth [54, 55]. Increased risk of development of cancers has been determined via epidemiological studies and meta-analyses to be related to alcohol consumption $[56,57]$. The risk spectrum from light drinking to chronic alcohol consumption has been explored and a significant risk attributable to alcohol has been established for various primary cancers $[58,59]$. 
In contrast to the known role of alcohol consumption in the development of primary tumors, whether or not alcohol exposure increases the risk to metastatic cancer distal from the primary site is unclear. Since cancer morbidity and mortality is largely attributable to metastases, rather than primary tumors, per se, the mechanisms by which alcohol influences metastasis requires further investigation. There are some clinical data that support the hypothesis that alcohol exposure increases the risk of cancer metastasis. In animal models, alcohol exposure increases the incidence of hematogenous metastases [60]. Many primary cancers commonly metastasize to the liver at a higher proportion than almost all secondary sites save lymph nodes, including breast, colon/colorectal, lung, ovarian, and neuroendocrine tumors [61, 62]. Hepatic metastases from colorectal cancer in particular are a significant clinical problem due to the frequency of synchronous lesions between the bowel and the liver [ 63 , 64]. In epidemiological studies, alcohol consumption has been identified as a significant independent risk factor for the development of colorectal liver metastases (CRLM) [65]. Retrospective analyses of clinical pathological reports have demonstrated a positive correlation between alcohol consumption and metastatic potential and patient outcomes [50,66]. However, whether this is a direct effect of alcohol consumption, or an indirect effect via increasing the risk of a primary tumor, is unclear. Furthermore, the specific mechanisms driving this correlation have yet to be elucidated and little is known about subclinical changes to microenvironment that influence organotropism of circulating tumor cells [67]. 
Chronic hepatic inflammation promotes dramatic remodeling of the liver ECM, which is an important factor in both fibrotic disease and cancer, contributing to tumor progression and metastasis $[68,69]$. The long-term efficacy of cancer therapeutics is often limited by metastatic disease, and often metastases exhibit selective tropism to the liver, particularly from primary lung and colorectal cancers. Although the mechanisms of metastatic organotropism are poorly understood, it is clear that communication between cancer cells and the target microenvironment are key. Indeed, several of the hallmarks of metastasis, such as proliferation, angiogenesis and apoptosis, are hypothesized to be mediated by altered integrin signaling $[17,18]$. Interestingly, alcohol consumption is a known risk factor for increasing metastasis to the liver, suggesting that early subclinical alcohol-mediated liver ECM remodeling may impact seeding and colonization of hepatic metastases [70].

In theory, integrins are attractive target because blocking their receptor function, or downstream signaling could prevent important steps in tumor development and progression, such as matrix adhesion and their ability to migrate. Integrin inhibition has been used as a targeted therapy against liver metastases, such as with endostatin and snake venom components [71, 72]. While these studies are critical to creating more efficacious targeted diagnostics and therapeutics for metastatic disease, a more efficient approach may be to target the integrin profile. As there are multiple components that can make up the dimeric integrin cell surface receptors, there exist multiple probabilities for combinations of these monomers, dependent typically on resident tissue and 
intended function $[46,73]$. However, as there is a sophisticated coordination of integrin-ECM interactions that often involve a significant amount of redundancy due to overlapping functionality, consideration of this complex tableau of integrin expression and downstream consequences is necessary for development of optimally efficacious therapies. Additionally, since many of the major class integrins, i.e. those consisting of the $\beta 1$-subunit, are ubiquitous and bind to most of the widespread group of ECM proteins (e.g., collagens, fibronectin, galectin), off-target effects of such therapies are a significant obstacle for these pharmaceuticals $[74,75]$. Anti-integrin therapies therefore have had some success but it is clear that various integrins mediate the steps of the metastatic cascade in the liver, so targeting them in a composite manner may be more effective therapeutically.

6. Macrophages and the cancer microenvironment Macrophages are a key liver cellular population, critical for tissue homeostasis and regulating injury and repair responses [76]. Both resident macrophages, i.e. Kupffer cells, and those derived from monocytic precursors are present in hepatic tissue. Kupffer cells are involved in metabolism, phagocytosis of bacterial and cellular debris, and maintaining hepatic homeostasis. Once activated, these cells recruit immune cells to liver, mediating inflammation, fibrosis, angiogenesis, and tumor progression. In both primary liver cancer and in hepatic metastases, the interactions of liver macrophages and tumor cells directly influence the preponderance of tumor-associated 
macrophages and their ability to modulate cancer-related inflammation to facilitate tumor progression [77].

The tumor microenvironment (TME) is now recognized as an important facet of carcinogenesis and progression through advanced stages of disease. Key players in this setting include host immune cells, namely macrophages, lymphocytes, and neutrophils [78-81]. Structural elements such as the ECM, fibroblasts, and vasculature also play specific, tissue-dependent roles. The inflammatory modulator in the setting of tumor interactions with the ECM is most often macrophages, recruited from circulating monocytes, which undergo differentiation and polarization according to cytokines released in the microenvironment that take direct or indirect action to influence remodeling [8287] angiogenesis and proliferation. Indeed, cross-talk between tumor-associated macrophages (TAMs) and cancer cells is critical to determining the balance between tumor cell death and tumor promotion [87]. Cell-cell signaling, via cytokine secretion, can inform the local immune response; nutrient availability, oxygenation, and tumor cell metabolism contribute to the cacophony of signaling from cells that redirects polarization of macrophages to suit the needs of the tumor, often resulting in their unwitting participation in tumor progression [88-90]. These are all critical processes that are often co-opted by tumor cells to promote growth, invasion, and extravasation, via repolarization/reprogramming of macrophages and remodeling of the extracellular matrix.

As macrophages are the key stewards of all stages of tumor progression, including metastasis, an entire field has burgeoned around their study and 
determining the capacity of their dual pro- and sometimes anti-tumor activity. In breast cancer, TAMs have been shown to facilitate cancer invasiveness via ECM degradation, promote angiogenesis, and suppress proinflammatory/tumoricidal T-cell responses $[83,91]$. Proinflammatory-polarized TAMs in large B cell lymphoma promote tumor progression by remodeling the ECM via fibronectin and collagen I degradation [92]. In colorectal carcinoma, they produce growth factors and inflammatory mediators and are clinical biomarkers of poor prognosis $[93,94]$. It is clear that TAMs and the ECM do not exert their influence on tumor progression in isolation, so development of a system by which to manage clinical surveillance of macrophages and target their actions on the ECM could be a key microenvironmental interaction for experimental analysis and theranostic interventional targeting [77].

7. Statement of goals

Exploration of the links between ECM composition and in particular the cell signaling behaviors influenced by pathological remodeling of the extracellular matrix has become an indispensable tenet of cancer research. Indeed, the "seed \& soil" hypothesis, one of the longest-surviving in medical literature, presumes a chicken \& egg paradigm in which either the tumor itself, the "seed," is the determinant in oncogenesis and metastatic spread; or, instead the "soil," that is, the primary tissue or metastatic target organ and the composition and characteristics thereof guide and dictate the seeding of tumor cells. This theory succinctly encapsulates the complexity of the influences the ECM effects on 
microenvironmental cues that drive the haywire functions that facilitate neoplastic spread. It is now clear that the seed, soil, and the entire milieu of secreted molecules, sequestered growth factors, and inevitable immune involvement all act in finely orchestrated concert to balance normal repair with injury and insult, including and up to the point when properties of each are exploited by cancer cells to promote their own survival and proliferation.

As discussed in this chapter, the Aims of this proposal will have a significant impact on the modeling of growth and neoangiogenesis of tumor cells in response to transitional fluctuations in abundance of chemotaxed and haptotaxed ECM components in the target organ. Our goal is to establish a composite profile of integrin binding to cognate ligands expressed in ECM based on quantitative analysis of the injured extracellular liver matrisome. Determining the pathological mechanisms by which alcohol influences hepatic metastases could help stratify a patient population which may have underlying ALD that contributes to metastatic seeding to the liver. Additionally, via surveillance of the phenotypic profile of macrophages in the tumor microenvironment, we hope to develop a 2D model to visualize tumors and macrophages, demonstrating that the tumor cells, as the "seed", here have the ability to exert effects on local macrophages, thereby indirectly affecting the "soil" to favor their attachment and proliferation. This new approach, leveraging integrated data and systems biology, could potentially lead to lead to increased precision in integrin- or immunetargeted prevention and treatment strategies. 


\section{B. Aims and proposals}

1. To computationally predict integrin binding kinetic profile based on experimental data analysis of integrin-mediated changes to the ECM

This dissertation proposes to utilize mathematical modeling to systematically explore changes in hepatic phenotype driven by altered ECMintegrin interactions. In attempt to recapitulate integrin receptor binding kinetics, we will develop a divalent receptor kinetic model that employs a simple integrin clustering scheme to determine binding affinity of each of the various ECM proteins to their various cognate integrin receptors. Proteomic data from a mouse model of hepatic fibrosis induced by carbon tetrachloride $\left(\mathrm{CCl}_{4}\right)$ exposure will be used to establish model parameters to simulate ECM binding and compare kinetics to homeostatic liver controls. This well-characterized in vivo model exhibits changes in integrin signaling that can be used to develop and train the in silico model, along with information from published literature on known binding rate constants for specific integrin receptors.

2. Characterizing macrophage polarization in response to treatments that promote liver injury in vivo

The purpose of the current study will be to evaluate the effect of arsenic on polarization of bone-marrow derived macrophages (BMDMs), as macrophage Th-1-like and Th-2-like responses are critical components of innate immunity. Macrophages have a critical influence on the inflammatory microenvironment in 
HCC [95]. In rodent studies, low level chronic exposure to inorganic arsenic induced malignancy and hepatocarcinogenesis. Arsenic trioxide has been shown to induce growth inhibition and apoptosis in human hepatocellular carcinoma cell lines, but not have sustained clinical effect in advanced or metastatic HCC [96]. Characterizing the effects of arsenic on macrophage behavior and, in turn, of macrophage polarization changes on tumor progression is the ultimate goal of this work. Polarized macrophages will be surveyed to determine modulation of macrophage populations following stimulation from their naïve state. Characterization of macrophage populations in response to various polarizing/depolarizing stimuli will provide a framework to explore molecules that modulate the inflammatory response, as well as assessing the conditions under which polarization switching begins to shift polarization differentially within a colony of differentiated macrophages. These data will be used to set experimental conditions for macrophages utilized in an indirect co-culture assay with both primary and metastatic tumor cells, to evaluate the potential for tumor signaling to influence polarization switching to co-opt macrophages in service of the proliferation of the tumor.

3. Evaluate perturbations to immunomodulatory stimuli and the extracellular matrix in the tumor microenvironment via a spatio-temporal mathematical model of tumor growth

This aim seeks to model the effects of ECM density and tumormacrophage interactions within the tumor microenvironment using in vitro data on tumor cell migration and cytokine levels measured in a transwell indirect co- 
culture of macrophages and tumor cells. These data will be used to set the parameters of a 2D tumor growth model within a transitionally-altered ECM scaffold and visualizing the distribution of heterogeneous macrophage populations clustered around the tumor and its nascent angiogenic vessels. This will determine if tumor cells have potential ability to exert immunomodulatory effect on local macrophages to induce their influence on ECM turnover, creating a more favorable microenvironment for expansion of tumor micronodule to a proangiogenic, proliferating cell cluster.

Overall aim of this dissertation

The overarching goals of the current work is to fill key gaps in current understanding of alcohol consumption and the risk of metastasis to liver. Considering the evidence this research group has compiled confirming that the hepatic matrisome responds dynamically to injury, an altered extracellular matrix (ECM) profile appears to be a key feature of pre-fibrotic inflammatory injury in the liver. This group has demonstrated that the hepatic ECM responds dynamically to alcohol exposure in particular, sensitizing the liver to LPS-induced inflammatory damage [45]. Although the study of alcohol in its role as a contributing factor to oncogenesis and metastatic progression has not been extensively investigated in basic science, it is clear from numerous clinical reports and meta-analyses that it would be a benefit to patients to understand the complexity of their comorbidities on a molecular level. It is well recognized that intramural research efforts are needed to understand the associated pathologies. 
Using signatures of liver ECM to predict cell surface integrin binding profile can establish personalized data that can be used clinically to determine therapeutic targets.

This work will establish a novel approach that explores systems biology data with the use of proteomics, along with a mathematical modeling strategy, focusing on the dynamics and kinetics of system component interactions. We will use the compiled proteomic data on ECM proteins that arise in experimental ALD to model and identify integrins that may favor metastasis. In order to tackle these big data, we employ a system-based approach integrating in silico, in vitro, and in vivo modeling. This project builds on the goals of the previous work in the Arteel lab, and proposes to continue exploring the overall hypothesis that alcoholrelated changes to the hepatic mastrisome (and subsequent alterations of integrin signaling) alters the hepatic phenotype. New exciting questions and cutting-edge modeling will be employed to use the information already attained in that project to leverage new clinically-relevant questions. This work will not only establish a novel approach, but also potentially identify new therapies that could be employed to prevent metastatic cancer to the liver.

This new approach leveraging integrated data could potentially lead to identifying new therapies that could be employed to prevent metastatic cancer to the liver. We hypothesize that transitional alterations to the hepatic matrisome create a more favorable environment for dissemination of metastases to the liver, and that specific integrins mediate these modifications. Alcohol-induced 
transitional remodeling of the hepatic ECM enhances metastatic seeding via integrin-mediated tumor-ECM interactions. 


\section{CHAPTER II}

\section{EXPERIMENTAL PROCEDURES}

\section{A. Animals and Treatments}

Mice were housed in a pathogen-free barrier facility accredited by the Association for Assessment and Accreditation of Laboratory Animal Care, and procedures were approved by the University of Louisville's Institutional Animal Care and Use Committee.

1. Animal sacrifice, tissue collection and storage

Prior to sacrifice, mice were anesthetized by injection of a ketamine $\mathrm{HCl} / x y l a z i n e ~ s o l u t i o n ~(100 / 15$ mg/kg i.m.; Sigma-Aldrich, St. Louis, MO). Animals were exsanguinated, allowing for blood collection from the vena cava; citrated plasma samples were stored at $-80^{\circ} \mathrm{C}$. Liver tissues were divided for snap freezing in liquid nitrogen, fixation in $10 \%$ neutral buffered formalin, or embedding in frozen specimen medium (Tissue-Tek OCT compound, Sakura Finetek, Torrance, CA); small samples are collected in RNA STAT-60 (Tel-Test, Inc., Friendswood, TX) to collect total RNA via chloroform:phenol separation (see section $\mathrm{D}$ for additional details). 


\section{BMDM Collection}

To harvest BMDMs, mice were anesthetized with ketamine/xylazine (100/15 mg/kg i.p.) and sacrificed by exsanguination. Bone marrow cells were flushed from tibiae and femora of sacrificed mice using ice cold PBS, and then pooled from each cohort (4-6 mice) for propagation in cell culture using endotoxin-free RPMI 1640 medium supplemented with 10\% fetal bovine serum (FBS; Gemini Bio-products, West Sacramento, CA) and $100 \mathrm{U} / \mathrm{mL}$ penicillin:100 $\mu \mathrm{g} / \mathrm{mL}$ streptomycin (GE Health care, Wauwatosa, WI), with macrophage colony stimulating factor (M-CSF) supplementation via conditioned media from L-929 cells to induce differentiation into macrophages.

\section{Lieber-DeCarli alcohol diet model}

Weanling C57BL/6J mice were obtained from the Jackson Laboratory (Bar Harbor, ME) and, following housing acclimation, mice were maintained on LieberDeCarli diet (Dyets, Inc., Bethlehem, PA), with either ethanol supplementation, or as pair-fed isocaloric control diet supplemented with only maltose-dextrin. Animals were housed in pairs and held in colony within a room where the ambient temperature was $75{ }^{\circ} \mathrm{F}$ to prevent hypothermia. Animals accessed diet via vacuum feeding tubes in which diet was replenished daily at 5:00 pm and consumption per cage was recorded. Following the initial two days of diet acclimation, wherein both cohorts were provided control isocaloric diet, the ethanol cohort received diet with increasing concentration of ethanol over a twoweek period, until reaching the intended experimental concentration of $6 \%$ 
( $\mathrm{vol} / \mathrm{vol})$ for the last three weeks of diet maintenance. That is, the ethanol cohort diet was comprised of $0 \%$ ethanol for the two-day acclimation period, $1 \%$, and then $2 \%$ for two days each, $4 \%$, and then $5 \%$ for one week each, then finally $6 \%$ feeding for the final three weeks. Animals were then sacrificed and liver tissue harvested for histological processing and ECM extraction.

\section{B. Histology}

1. General morphology

Liver tissues were either formalin fixed and embedded in paraffin (FFPE), or embedded frozen in OCT prior to cutting at either $5 \mu \mathrm{m}$ or $8 \mu \mathrm{m}$, respectively, and then mounted onto charged glass slides. FFPE sections were processed in Citrisolv (Thermo Fisher Scientific, Waltham, MA) and rehydrated via incubation in graded ethanol concentrations. Sections were stained with hematoxylin and eosin (H\&E), Sirius red, or Mason's Trichrome, before mounting with Permount (Thermo Fisher, Waltham, MA).

\section{Proteomics}

1. 3-step ECM extraction

Sample preparation and wash: Snap-frozen liver tissue $(75-100 \mathrm{mg})$ was immediately added to ice-cold phosphate-buffered saline ( $\mathrm{pH}$ 7.4) wash buffer containing commercially available protease and phosphatase inhibitors (Sigma Aldrich) and $25 \mathrm{mM}$ EDTA to inhibit proteinase and metalloproteinase activity, respectively. While immersed in wash buffer, liver tissue was diced into small 
fragments and washed five times to remove contaminants. Between washes, samples were pelleted by centrifugation at $10,000 \times \mathrm{g}$ for $5 \mathrm{~min}$ and wash buffer was decanted.

$\mathrm{NaCl}$ extraction: Diced samples were incubated in 10 volumes of $0.5 \mathrm{M}$ $\mathrm{NaCl}$ buffer, containing $10 \mathrm{mM}$ Tris $\mathrm{HCl}(\mathrm{pH} 7.5)$, proteinase/phosphatase inhibitors, and 25 mM EDTA. The samples were gently mixed on a plate shaker (800 rpm) overnight at room temperature. The following day, the remaining tissue pieces were pelleted by centrifugation at $10,000 \times \mathrm{g}$ for $10 \mathrm{~min}$. The supernatant was saved and labeled as the $\mathrm{NaCl}$ fraction.

SDS extraction: The pellet from the $\mathrm{NaCl}$ extraction was subsequently incubated in 10 volumes (based on original weight) of a $1 \%$ SDS solution, containing proteinase/phosphatase inhibitors and $25 \mathrm{mM}$ EDTA. The samples were gently mixed on a plate shaker ( $800 \mathrm{rpm}$ ) overnight at room temperature. The following day, the remaining tissue pieces were pelleted by centrifugation at $10,000 \times \mathrm{g}$ for $10 \mathrm{~min}$. The supernatant was saved and labeled as the SDS extract.

Guanidine $\mathrm{HCl}$ extraction: The pellet from the SDS extraction was incubated with five volumes (based on original weight) of a denaturing guanidine buffer containing $4 \mathrm{M}$ guanidine $\mathrm{HCl}(\mathrm{pH} 5.8), 50 \mathrm{mM}$ sodium acetate, $25 \mathrm{mM}$ EDTA, and proteinase/phosphatase inhibitors. The samples were vigorously mixed on a plate shaker at $1200 \mathrm{rpm}$ for $48 \mathrm{~h}$ at room temperature; vigorous shaking is necessary at this step to aid in the mechanical disruption of ECM components. The remaining insoluble components were pelleted by 
centrifugation at $10,000 \times \mathrm{g}$ for 10 minutes. This insoluble pellet was retained and solubilized as described below. The supernatant was saved and labeled as the $\mathrm{GnHCl}$ fraction.

Deglycosylation and solubilization: The supernatants from each extraction were desalted using Zeba Spin columns (Pierce) according to manufacturer's instructions. The desalted extracts were then mixed with five volumes of $100 \%$ acetone and stored at $-20^{\circ} \mathrm{C}$ overnight to precipitate proteins. The precipitated proteins were pelleted by centrifugation at $16,000 \times \mathrm{g}$ for $45 \mathrm{~min}$. Acetone was evaporated by vacuum drying in a RotoVap for one hour. Dried protein pellets were resuspended in $500 \mu \mathrm{L}$ deglycosylation buffer $(150 \mathrm{mM} \mathrm{NaCl}, 50 \mathrm{mM}$ sodium acetate, $\mathrm{pH}$ 6.8, $10 \mathrm{mM}$ EDTA, and proteinase/phosphatase inhibitors) that contained chondroitinase ABC (P. vulgaris; $0.025 \mathrm{U} /$ sample), endo-betagalactosidase (B. fragilis; $0.01 \mathrm{U} /$ sample) and heparitinase II (F. heparinum; $0.025 \mathrm{U} /$ sample). Samples were incubated overnight at $37^{\circ} \mathrm{C}$; those containing the pellet remaining after the guanidine $\mathrm{HCl}$ step received $20 \mu \mathrm{L}$ DMSO for solubilization. Protein concentrations were estimated by absorbance at $280 \mathrm{~nm}$ using bovine serum albumin (BSA) in deglycosylation buffer for reference standards.

2. LC-MS/MS analysis of samples

Sample cleanup and preparation for liquid chromatography: Pooled samples in deglycosylation buffer were thawed to room temperature and clarified by centrifugation at $5,000 \times \mathrm{g}$ for $5 \mathrm{~min}$ at $4^{\circ} \mathrm{C}$. Samples were reduced by adding 1 
M DTT to $50 \mu \mathrm{L}(25 \mu \mathrm{g})$ of each sample and then incubating at $60^{\circ} \mathrm{C}$ for $30 \mathrm{~min}$ before addition of $8 \mathrm{M}$ urea in $0.1 \mathrm{M}$ Tris- $\mathrm{HCl}(\mathrm{pH}$ 8.5) was added to each sample. Each reduced and diluted sample was digested with a modified FilterAided Sample Preparation (FASP) method. Recovered material was dried in a SpeedVac and redissolved in $200 \mu \mathrm{L}$ of $2 \% \mathrm{v} / \mathrm{v}$ acetonitrile (ACN)/0.4\% formic acid (FA). The samples were then trap-cleaned with a C18 PROTOTM $300 \AA$ Ultra MicroSpin Column (The Nest Group). The sample eluates were incubated at $-80^{\circ} \mathrm{C}$ for $30 \mathrm{~min}$, dried in a SpeedVac, and stored at $-80^{\circ} \mathrm{C}$. Before liquid chromatography, dried samples were warmed to room temperature and dissolved in $2 \% \mathrm{v} / \mathrm{v}$ ACN $/ 0.1 \%$ FA to a final concentration of $0.25 \mu \mathrm{g} / \mu \mathrm{L}$. A volume of $16 \mu \mathrm{L}(4 \mu \mathrm{g})$ of sample was injected into the Orbitrap Elite.

Liquid Chromatography: Dionex Acclaim PepMap 100, $75 \mu \mathrm{M}$ × 2 cm nanoViper (C18, $3 \mu \mathrm{m}, 100 \AA$ ) trap and Dionex Acclaim PepMap RSLC, $50 \mu \mathrm{M}$ x $15 \mathrm{~cm}$ nanoViper (C18, $2 \mu \mathrm{m}, 100 \AA)$ separating column were used. An EASY nLC (Thermo) UHPLC system was used with mobile phase buffer $A(2 \% \mathrm{v} / \mathrm{v}$ acetonitrile/0.1\% v/v formic acid), and buffer B (80\% v/v acetonitrile/0.1\% v/v formic acid). Following injection of the sample onto the trap, separation was accomplished with a 140 min linear gradient from $0 \%$ B to $50 \%$ B, followed by a 30 min linear gradient from $50 \%$ B to $95 \%$ B, and lastly a 10 min wash with $95 \%$ B. A 40-mm stainless-steel emitter (Thermo P/N ES542) was coupled to the outlet of the separating column. A Nanospray Flex source (Thermo) was used to position the end of the emitter near the ion transfer capillary of the mass 
spectrometer. The ion transfer capillary temperature of the mass spectrometer was set at $225^{\circ} \mathrm{C}$, and the spray voltage was set at $1.6 \mathrm{kV}$.

Mass Spectroscopy: An Orbitrap Elite - ETD mass spectrometer (Thermo) was used to collect data from the LC eluate. An Nth Order Double Play with ETD Decision Tree method was created in Xcalibur v2.2. Scan event one of the method obtained an FTMS MS1 scan for the range $300-2000 \mathrm{~m} / \mathrm{z}$. Scan event two obtained ITMS MS2 scans on up to ten peaks that had a minimum signal threshold of 10,000 counts from scan event one. A decision tree was used to determine whether collision induced dissociation (CID) or electron transfer dissociation (ETD) activation was used. An ETD scan was triggered if any of the following held: an ion had charge state 3 and $\mathrm{m} / \mathrm{z}$ less than 650 , an ion had charge state 4 and $\mathrm{m} / \mathrm{z}$ less than 900 , an ion had charge state 5 and $\mathrm{m} / \mathrm{z}$ less than 950 , or an ion had charge state greater than 5; a CID scan was triggered in all other cases. The lock mass option was enabled ( $0 \%$ lock mass abundance) using the $371.101236 \mathrm{~m} / \mathrm{z}$ polysiloxane peak as an internal calibrant.

Proteome Data Analysis: Proteome Discoverer v1.4.0.288 was used to analyze the data collected by the mass spectrometer. The database used in Mascot v2.4 and SequestHT searches was the 6/2/2014 version of the UniprotKB Mus musculus reference proteome canonical and isoform sequences. In order to estimate the false discovery rate, a Target Decoy PSM Validator node was included in the Proteome Discoverer workflow. The Proteome Discoverer analysis workflow allows for extraction of MS2 scan data from the Xcalibur RAW file, separate searches of CID and ETD MS2 scans in Mascot and Sequest, and 
collection of the results into a single file (.msf extension). The resulting .msf files from Proteome Discoverer were loaded into Scaffold $Q+S$ v4.3.2. Scaffold was used to calculate the false discovery rate using the Peptide and Protein Prophet algorithms. The results were annotated with mouse gene ontology information from the Gene Ontology Annotations Database.

\section{Computational Modeling}

1. Kinetic simulations

Computer simulations were run using Spyder for Tellurium software version 2.3.5.2; Python version 2.7 [97]. Binding curves were plotted using SigmaPlot 13.0. Model was initialized using ligand concentrations from proteomic analysis and initial integrin concentrations were derived from published values. Ligand concentration was developed by collapsing the fractionated sample data using MudPIT functionality in Scaffold. Rappsilber et al. defined protein abundance index (PAI) for estimation of absolute protein abundance, and Ishihama et al. report that the emPAl, exponential PAI, is approximately proportional to protein abundance. Using the emPAl quantitative method, proteomic output was normalized by the tissue loading concentration of 0.25 $\mu \mathrm{g} / \mu \mathrm{L}$; these values for concentration were then divided by the molecular weight of the protein to convert to molar concentration. Kinetic rates listed in Tables 2 were used to calculate microrate parameters relative to the established binding rates from literature; where exact microrates were unavailable, rates were estimated from various published literature sources. Equilibrium constant table 
relates the equilibrium constants of the system relative to initial integrin complex formation, such that subsequent binding and clustering steps produce cooperativity when simulated in these proportions. Sensitivity analysis was performed by varying levels of integrin receptor concentration in 10-fold increments, to explore binding when surface membrane integrin receptor expression is upregulated or downregulated as a consequence of disease state or in response to microenvironmental fluctuations.

\section{Tumor model simulations}

Tumor model simulates effect of ECM production/degradation ratios and the interaction with macrophage polarization to M1- and M2-activated subtypes on tumor growth and neoangiogenesis. Mathematical model is application of model developed in Leonard et al. and extended by Mahlbacher et al. A detailed summary is provided in Chapter V, Section B.6-7.

\section{E. RNA Isolation and Quantitative Reverse-Transcription Polymerase Chain $\underline{\text { Reaction }}$}

The mRNA expression of select genes was detected by quantitative reverse-transcriptase polymerase chain reaction (qPCR), as previously described [98]. Total RNA was extracted by a guanidinium thiocyanate-based method (RNA STAT 60; Tel-Test, Friendswood, TX). RNA concentrations were determined spectrophotometrically and $1 \mu \mathrm{g}$ of total RNA was reverse transcribed using qScript cDNA SuperMix (Quanta Biosciences, Gaithersburg, MD). PerfeCTa 
qPCR Fast Mix (Applied Biosystems) was used for the PCR reaction, carried out on the ABI StepOne Plus (Applied Biosystems). The comparative CT method was used to determine fold changes in mRNA expression compared to an endogenous reference gene (18S). This method determines the amount of target gene, normalized to an endogenous reference and relative to a calibrator (2$\Delta \Delta \mathrm{Ct})$.

\section{F. Microarray analysis of miRNA}

1. Biostatistical analysis

Total RNA from cells were isolated using mirVana total RNA isolation kit (Life Technologies, Carlsbad, CA) according to the manufacturer's guidelines. RNA concentrations were determined spectrophotometrically using a Nanodrop ND-1000 (Thermo Fisher Scientific, Grand Island, NY). The integrity of total RNAs was assessed using Agilent 2100 Bioanalyzer (Agilent, Santa Clara, CA). $350 \mathrm{ng}$ of total RNA was labeled with Biotin using a 3DNA Array Detection FlashTag $^{\mathrm{TM}}$ Biotin HSR kit (Affymetrix, Santa Clara, CA, U.S.) following the manufacturer's protocol, being subsequently hybridized overnight. The GeneChip $₫$ miRNA 4.0 arrays, containing 30,424 total mature miRNA probe sets were washed and stained using the Affymetrix GeneChip Hybridization Wash and Stain Kit and were then scanned with the Affymetrix GeneChip Scanner 3000 7G (Affymetrix). Raw intensity scores were imported into Partek Genomics Suite 6.6 (Version 2.16.1441; Partek, Inc., St. Louis, MO, USA) and normalized on a gene level using the standard Robust Multi-array Average (RMA) algorithm 
for normalization and background correction. Volcano plot and heatmap generated in $\mathrm{R}$ (Version 3.3.2, $\mathrm{R}$ Core Team, 2016) via data analysis using the pd.mirna.4.0 Bioconductor package [99] and linear contrast with the limma package [100]. The volcano plot stratifies array data with significance $(p=0.05)$ versus $\log 2$ fold change $(\log 2 \mathrm{FC} ;>0.301$ or $<-0.301)$ on the $y$ - and $x$-axes, respectively. As follows, the representative scatter plot visualizes miRNAs to identify those differentially expressed with respect to direction of fold change. The heatmap visualizes miRNAs with statistically significant expression changes across all treatments; hierarchical clustering analysis was used to identify patterns of miRNA expression in the As(III), LPS, and LPS+As(III) treatment groups. Linear discriminant analysis Effect size (LEfSe; [101]) analysis was used in parallel to identify differentially expressed miRNAs by using class comparison methods to predict significant biomarkers.

3. Enrichment tools

Raw data CEL-files for analysis of the miRNA arrays were produced with Affymetrix GeneChip Command Console Software Version 4.0 (Affymetrix). Partek Genomics Suite software (Version 6.14.0923; Partek, Inc.) was used for further analysis. CEL-files were imported including control and interrogating probes, and the arrays were normalized using quantile normalization. Probeset summarization was done using Median Polish. Probe values were log2transformed. One-way analysis of variance (ANOVA) was performed to detect 
differential miRNA expression between the groups, and Fisher's Least Significant Difference (LSD) was used as the contrast method.

4. Gene ontology and pathway analysis

TargetScan (Release 7.1: http://www.targetscan.org; [102] was used to predict biological targets of identified miRNAs via seed region matching; database accessed on 9/18/2016. miRBase database (miRBase 21 release; [103-105] ) was utilized for mining miRNA sequencing and annotation data. Ontological classification and functional enrichment analysis to identify significantly over-represented biological processes were performed using the database for annotation, visualization, and integrated discovery (DAVID) v6.7, and pathways in each cluster were classified; functional annotation completed 4/13/2016. A modified Fisher's exact test/EASE (Enrichment) Score was utilized to calculate the $p$-values; threshold was set at $p<0.05$ and fold enrichment $>2$. Gene Ontology (GO) enrichment analysis tool corrected using the Bonferroni method. To identify enrichment terms associated with miRs of interest, gene lists were uploaded to the DAVID web site using the complete mouse genome as background. Significantly enriched functional groups were ranked using the Functional Annotation Clustering tool set to the highest classification stringency, with the $p$-value set at 0.05 . Protein domain annotations were derived from Pfam database, a database of evolutionarily conserved protein domain coordinates (Bateman et al., 2000); BioCarta and KEGG tools were used to visualize gene pathway mapping. 
miRNA expression analysis uploaded into the Ingenuity Pathway Analysis Knowledge Base (IPA®, Version 43605602, Qiagen, Redwood, CA) for pathway and gene otology analysis; Core analysis as well as comparative analysis was used with filters specific to inflammation, macrophages, and innate immunity. Toxicity functional analysis correlating pathological endpoints, as well as diseases and biofunctions were reported; database was queried on 9/7/2017. Fisher's exact test was used to find the selected significant miRNA expression changes with $\mathrm{FC}>2$ and $\mathrm{p}$ values $<0.05$, and network analysis was generated for interactions of the mapped genes of putative miRNA targets from expression data to the molecular relationships stored in the knowledge base. Additional detail on methodology and functionality can be obtained on the IPA website.

\section{Statistical analysis}

Flow cytometry and qPCR data results were reported as means \pm standard error mean (SEM). The comparative analysis of the results from various experimental groups with their corresponding controls was performed using SigmaStat for Windows (Systat Software, Inc., San Jose, CA). ANOVA followed by Tukey's post-hoc test was carried out to assess statistical significance between treatment groups. A $p$ value $<0.05$ was selected before the study as the level of significance for results.

G. In vitro cell analyses

1. Flow cytometry 
Cells were trypsinized, collected, washed in FAB buffer (1\% FBS in PBS), and blocked with Fc block (BD Biosciences, San Jose, CA) for 15 min prior to antibody labeling and subsequent FAB washes. Cells were stained with antibodies for 30 min at $4^{\circ} \mathrm{C}$ (CD11b-APC, F4/80-PE, CD11c-BV 421, CD206-BV 651; eBiosciences Inc., San Diego, CA). Cells were fixed with $2 \%$ paraformaldehyde (Alfa Aesar, Ward Hill, MA) for 15 min and resuspended in fresh FAB buffer prior to analysis. Data were acquired on a BD Fortessa flow cytometer using BD FACS Diva software (BD Biosciences), and all compensation and data analyses were performed using FlowJo software (FlowJo LLC, Ashland, OR). Compensation beads (eBiosciences Inc.) were used to balance multichannel emission spectra of the selected fluorochromes, in addition to fluorescence minus one (FMO) controls for gating. Unstained and isotype controls were used to exclude doublets and restrict gating to macrophage differentiated, $\mathrm{CD} 11 \mathrm{~b}^{+}+\mathrm{F} 4 / 80^{+}$cells.

2. BMDM cell culture and differentiation

Cells were harvested and isolated from animals as described in Chapter II, Section A.2. Following seven days of media-conditioning, differentiated cells were harvested and seeded at a density of $2.5 \times 10^{5} \mathrm{cells} / \mathrm{mL}$ in 6 -well tissue culture plates. After $24 \mathrm{~h}$, cells were exposed for $6-18 \mathrm{~h}$ to increasing concentrations of arsenic (as sodium arsenite, Sigma-Aldrich, St. Louis, MO) in the presence/absence of lipopolysaccharide (LPS; from E. coli serotype O55:B5; Sigma-Aldrich) or IL-4 (R\&D research; Minneapolis, MN) Ying et al., 2013. 


\section{MTT viability assay}

Cell viability was determined using the 3-(4,5-Dimethylthiazol-2-yl)-2,5diphenyltetrazolium bromide (MTT; Sigma-Aldrich) assay as described previously (Anwar-Mohamed et al., 2012) and validated by trypan blue (Sigma Aldrich) exclusion.

4. Transwell migration assay

Tumor cell lines designated WLA3 (derived from primary lung tumor in B6129S2/J mice) and WA8 (derived from lung to lymph node metastases in B6129S2/J mice presenting with primary lung tumors) were a kind gift from Dr. Monte Winslow at the Massachusetts Institute of Technology [106]. Cell lines were maintained with DMEM media with $10 \%$ FBS, and $100 \mathrm{U} / \mathrm{mL}$ penicillin:100 $\mu \mathrm{g} / \mathrm{mL}$ streptomycin at $1 \%$. Serum-starved tumor cells were seeded at concentration of $2 \times 10^{5}$ in transwells containing either lyophilized ECM (LECM) from control or ethanol-fed liver tissues, or control uncoated wells, with $2 \%$ FBS added to bottom receiver chamber as chemoattractant. After $48 \mathrm{~h}$, cells on lower surface of membrane were fixed with $4 \%$ paraformaldehyde, stained with $0.2 \%$ crystal violet in $100 \%$ ethanol, and counted manually, 10 fields per membrane, using automatic slide reader.

5. Tumor cell/macrophage indirect co-culture assay

Transwell culture plates were utilized to create an indirect co-culture environment between tumor cell lines (either primary or metastatic) and BMDMs. A porous $0.4 \mu \mathrm{m}$ transwell insert membrane was utilized to prevent migration of 
tumor cells from top chamber to bottom receiver well. Macrophages were first seeded to bottom receiver plate well at a density of $1 \times 10^{5}$ cells $/$ well and allowed to adhere overnight. Cells were then treated with either LPS to induce M1activation, or IL-4 for M2-activation; control cells were untreated naïve macrophages. After $24 \mathrm{~h}$, tumor cells were seeded to the top chamber insert at a density of $5 \times 10^{4}$ cells/insert; control inserts contained media only and no tumor cells. Following $48 \mathrm{~h}$ of indirect co-culture, cell supernatant was collected for further analysis.

6. ELISA

Cell culture supernatant from indirect co-culture transwell assays was evaluated using Quantikine ELISA kits for TNF- $\alpha$ and TGF $\beta-1$ (R\&D Systems, Minneapolis, MN) per manufacturer's instructions.

\section{H. Decellularized liver preparation}

1. Tissue preparation

Liver tissues were snap frozen upon collection. To prepare for decellularization, $600 \mathrm{mg}$ of frozen tissue was weighed and added to $45 \mathrm{ml}$ sterile 1X PBS in $50 \mathrm{ml}$ conical tubes. Tubes were put on shaker in cold room for gentle agitation overnight at $4^{\circ} \mathrm{C}$. Tissues were carefully removed from conical tubes with sterile forceps and transferred to new $50 \mathrm{ml}$ conical tube containing $0.1 \%$ EDTA in $10 \mathrm{mM}$ Tris $\mathrm{HCl}$ at $\mathrm{pH}$ 8.0. Tubes were shaken at room temperature for one hour, then carefully removed with forceps and transferred to new $50 \mathrm{ml}$ conical tube containing $0.1 \%$ SDS in $10 \mathrm{mM}$ Tris $\mathrm{HCl}$ at $\mathrm{pH}$ 8.0. Tubes were 
shaken at room temperature for $24 \mathrm{~h}$, then exchanged into fresh SDS buffer and shaken for another $24 \mathrm{~h}$ at room temperature.

After final $24 \mathrm{~h}$ of decellularization, tissues were washed three times by careful transfer to new $50 \mathrm{ml}$ conical containing sterile 1X PBS and gentle agitation for an hour. Tissues were then transferred to $1.5 \mathrm{ml}$ Eppendorf tubes, then centrifuged at $10,000 \times \mathrm{g}$ for $10 \mathrm{~min}$. PBS was decanted and tissues were frozen overnight at $-80^{\circ} \mathrm{C}$.

2. Lyophilization

Tissues were kept on dry ice until lyophilization RotoVap was initiated. Eppendorf tubes were opened and covered with parafilm, with small holes perforated using a small pipette tip. Tubes were placed inside lyophilization jar with cap open and parafilm opening exposed. Jar was attached to adaptor and secured. Lyophilization was initiated at a temperature of $-80^{\circ} \mathrm{C}$ and $30 \mathrm{mTorr}$ pressure, then evaporated for $48 \mathrm{~h}$. Upon completion of $48 \mathrm{~h}$ cycle, the pressure was slowly released from jar prior to its detachment from RotoVap apparatus. Tissue was hardened and white, then it was transferred to Eppendorf tube and ground with $10 \%$ pepsin in $0.1 \mathrm{M} \mathrm{HCl}$. Sample was diluted to final concentration of $0.2 \mathrm{mg} / \mathrm{ml}$ in $0.2 \mathrm{M}$ acetic acid.

\section{Culture plate coating}

To coat cell culture plates, uncoated 12-well plates were treated with 0.2 $\mathrm{mg} / \mathrm{ml}$ of lyophilized ECM in acetic acid, then incubated at $37^{\circ} \mathrm{C}$ for one hour. Wells were then washed three times with sterile 1X PBS prior to seeding cells. 


\section{CHAPTER III}

\section{MODELING THE KINETICS OF INTEGRIN RECEPTOR BINDING TO HEPATIC EXTRACELLULAR MATRIX PROTEINS ${ }^{1}$}

\section{A. Introduction}

The extracellular matrix $(\mathrm{ECM})$ consists of a broad range of components that interact bi-directionally with neighboring cells to create a dynamic and responsive microenvironment that regulates cell signaling, recruitment, and tissue function. The ECM not only provides structure and support for the cells in a tissue, but also acts as a reservoir for growth factors and cytokines and as a signaling mechanism by which cells can intercommunicate with their environment [107]. Quantitative and qualitative changes to the ECM structure and superstructure can impact overall health of the organ and organism. In particular, the hepatic ECM changes predominantly described in published literature occur in the context of hepatic fibrosis, which is characterized by robust scarring of the liver with collagen fibrils. However, the ECM of the healthy and fibrotic liver is significantly more diverse than collagen ECM. Recent studies also indicate that the hepatic ECM content changes dynamically in response to even acute stress and injury $[7,44,45]$. Furthermore, changes to the hepatic ECM may foster a

\footnotetext{
1 Published in Hudson SV et al. Scientific Reports 2017.
} 
'soil' that is conducive to cancer and metastasis. Although the concept that hepatic ECM changes drive hepatic dysfunction under several conditions is well understood, the mechanisms by which these effects are mediated are not. Integrins comprise a family of heterodimeric transmembrane glycoprotein receptors that facilitate key interactions between cells and the ECM $[16,108$, 109]. The binding of ECM ligands to integrins mediates critical processes, including cell adhesion, migration, proliferation, differentiation, inflammation and apoptosis. Indeed, several of the hallmarks of liver diseases and cancer (e.g., altered proliferation, angiogenesis and apoptosis) are hypothesized to be mediated via changes in ECM:integrin signaling [110]. Based on this assumption, integrins have become important therapeutic targets for diseases of dysregulation, including various cancers, fibrosis, and immune dysfunction. However, few integrin-based therapies have been effective to prevent and/or treat these diseases in the clinics. This limitation is likely due, at least in part, to an incomplete understanding of the complexity of the changes to integrin signaling under these dysregulated conditions.

The kinetics of ECM:integrin interactions are highly complex. Integrin receptors complexes are structured as non-covalently linked $\alpha$ and $\beta$ subunits, the various combinations of which contribute to diversity of receptors types [14] (Fig 1). The overall rate is not driven simply by ligand binding to the receptor, but also by clustering at focal adhesion points and an increase in avidity for binding additional ligand (i.e., positive cooperativity). Masson-Gandais et al. described a two-step model wherein the $\alpha$ subunit binds ligand first, influencing ligand 
recognition and determinant of association kinetics [111]. The $\beta$ subunit binds second, which creates bond stabilization and determines dissociation kinetics. Ligand binding to the extracellular domain activates the receptor and initiates its conformational changes to a high-affinity state $[112,113]$. This two-step process reflects a divalent kinetics model with the $\alpha$ subunit as the high affinity site, and the $\beta$ subunit as the low affinity site [114]. In addition to binding processivity of individual receptors, ligand binding to distinct integrins favors subsequent binding by other receptors (i.e. focal adhesion clustering). Furthermore, integrin receptors bind promiscuously to various ECM ligands, creating redundancy, competition and diversity in biofunctionality $[14,16,115]$. These complex interdependent factors affect the kinetics of ECM-integrin interactions in the intact organism. Promiscuity among the repertoire of ECM ligands and integrin receptors, particularly those with RGD-binding motifs, implies a differential pattern of binding relative to the amounts of substrate available. Elucidating these complex cell-ECM-driven pathological changes could lead to improved prognostics and clinical outcomes via more precise therapeutic management of the tissue microenvironment.

To explore these complex interactions, several mathematical descriptions of integrin binding have been reported with outputs related to spatial clustering and signal transduction, liver fibrosis and integrin-mediated cell haptotaxis [116118]. Although these models recapitulate certain aspects of ECM-integrin interactions, they typically focus on one ligand (e.g. collagen or fibronectin) as the ECM substrate. In this study, modeling of integrin receptor binding kinetics is 
reported that considers the divalent receptor characteristic and employs a simple model of integrin clustering. The kinetic indices of each integrin for each of its ligands were initially determined to establish a single-species integrin profile. Proteomic data were compiled that assess the liver ECM under homeostatic conditions as well as under experimental fibrosis. These proteomic analyses provided information on relative abundance of hepatic ECM components to calibrate substrate concentrations for the kinetic simulations. By testing homeostatic conditions against the experimental treatment models, how the integrin binding phenotype changes in response to injury could be determined and used to predict the ECM-integrin binding within the context of transitional tissue remodeling. 


\section{Scheme 3.1: Repertoire of alpha and beta integrin subtype dimerization pairings.}

This diagram delineates the 24 possible integrin dimer species, classified by substrate type. For this study, of the integrins relevant to the $\mathrm{CCl}_{4}$ model, the collagen-binding $\beta 1$ integrins and RGD-binding $\beta 1$ and $\beta 3$ receptors were evaluated. Binding here was treated as a two-step model with the a subunit binds ligand first, influencing ligand recognition and determinant of association kinetics. The $\beta$ subunit binds second, creating bond stabilization and determining dissociation kinetics [115], apropos to a divalent kinetics model with the $\alpha$ subunit being the high affinity site, and the $\beta$ subunit as the low affinity site. 


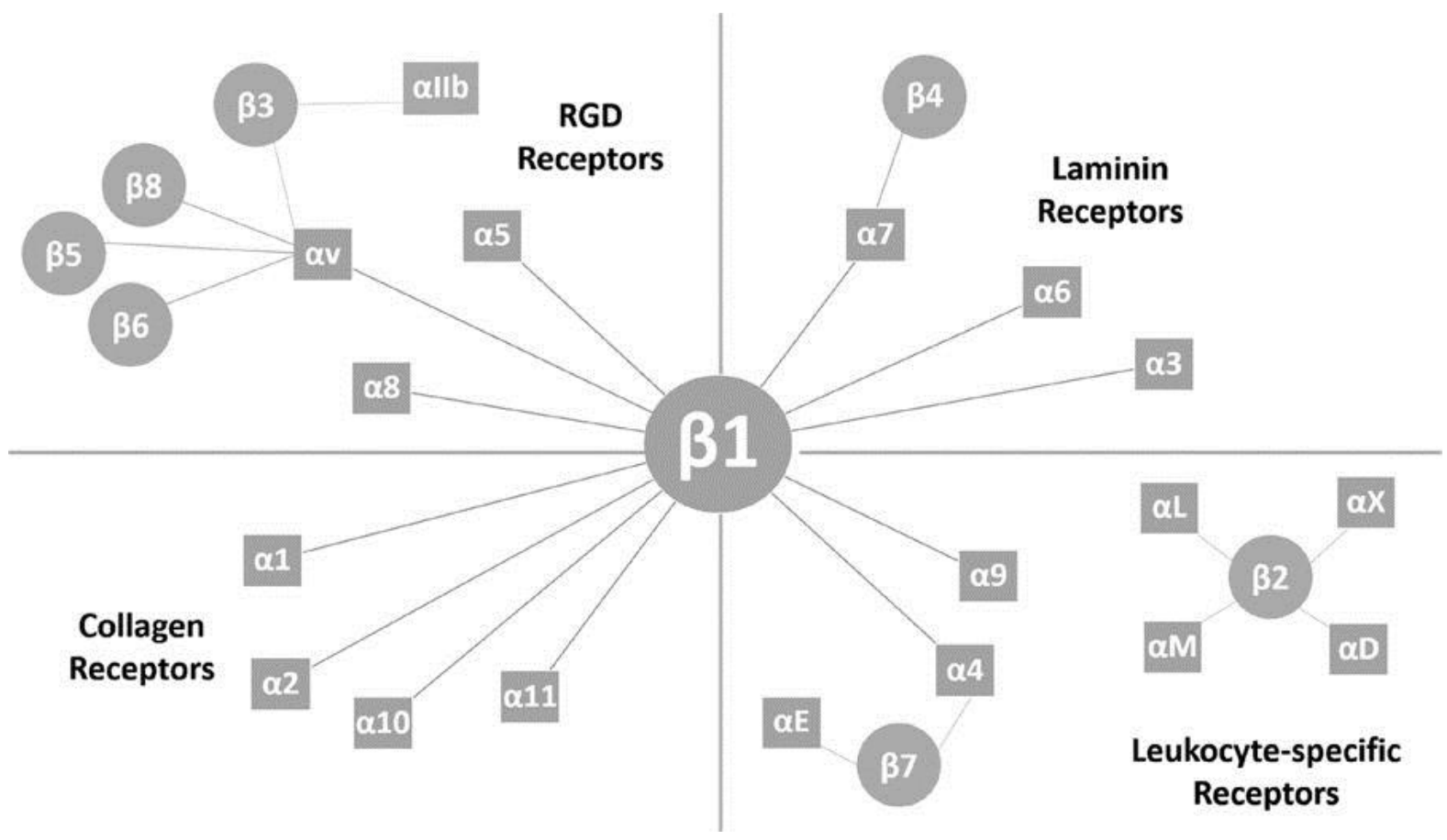




\section{B. Experimental Procedures}

1. Animals and treatments

Male C57BL/6J mice (4-6 w) were purchased from Jackson Laboratory (Bar Harbor, ME). Food and tap water were provided ad libitum. Mice were administered $\mathrm{CCl}_{4}$ (1 ml/kg i.p.; diluted 1:4 in olive oil; Sigma-Aldrich, St. Louis, MO) $2 \times / w k$ for 4 wk. Twenty-four hours after the last $\mathrm{CCl}_{4}$ administration, mice were anesthetized by injection of a ketamine $\mathrm{HCl} / \mathrm{xylazine}$ solution $(100 / 15 \mathrm{mg} / \mathrm{kg}$ i.m.; Sigma-Aldrich, St. Louis, MO). Other animals received the same dose of $\mathrm{CCl}_{4}$, but only once, and were sacrificed $12-72 \mathrm{~h}$ after intoxication. At sacrifice, tissue samples were collected as described in Chapter II, Section A.1.

\section{Histology}

Liver tissues frozen in OCT medium blocks were sectioned and stained with collagen type 5a1 (Abcam) and resolved with Alexa 488-tagged secondary antibody for immunofluorescence microscopy as described in Chapter II, Section B.3.

3. Proteomics

Proteome analysis was performed as described in Chapter II, Section C.

4. Computational Modeling 
First is considered the divalent receptor model which corresponds to ECM ligand binding of the $\alpha$ subunit occurring prior to the $\beta$ subunit $[46,119]$. This kinetic reaction is represented as shown in Scheme 3.2. 


\section{Scheme 3.2: Model description}

(a), mass-action kinetics scheme of species variables. Divalent receptors bind ligand sequentially to $\alpha, \beta$ subunits, with $\mathrm{K}_{\mathrm{i}}=k_{2} / k_{1}$ for equilibrium of initial binding event $\left(C_{m}\right)$ and $\mathrm{K}_{\mathrm{c}}=k_{4} / k_{3}$ for fully occupied ECMP-Integrin receptor complex $\left(C_{d}\right)$. The receptor aggregation scheme incorporates divalent binding and receptor pairing such that half/fully occupied receptors can aggregate only with an unbound receptor [aggregation equilibrium constant $\mathrm{K}_{\mathrm{a}}=k_{6} / k_{5}$ ]. Binding constants for an additional ECM ligand binding to unbound receptor in an aggregate pair are the same regardless of occupancy status of its paired receptor [population equilibrium constant is $\mathrm{K}_{\mathrm{p}}=k_{10} / k_{9}$ ]. The equilibrium constant for adding a second ECM ligand to a singly-bound receptor in any pair-configuration is $\mathrm{K}_{\mathrm{x}}=k_{8} / k_{7}$. Adapted from Wanant and Quon (2000)르. (b), model species description. 


$$
I+E \underset{k_{2}}{\stackrel{k_{1}}{\rightleftharpoons}} C_{m} \underset{k_{4}}{\stackrel{k_{3}}{\rightleftharpoons}} C_{d}
$$

(a)
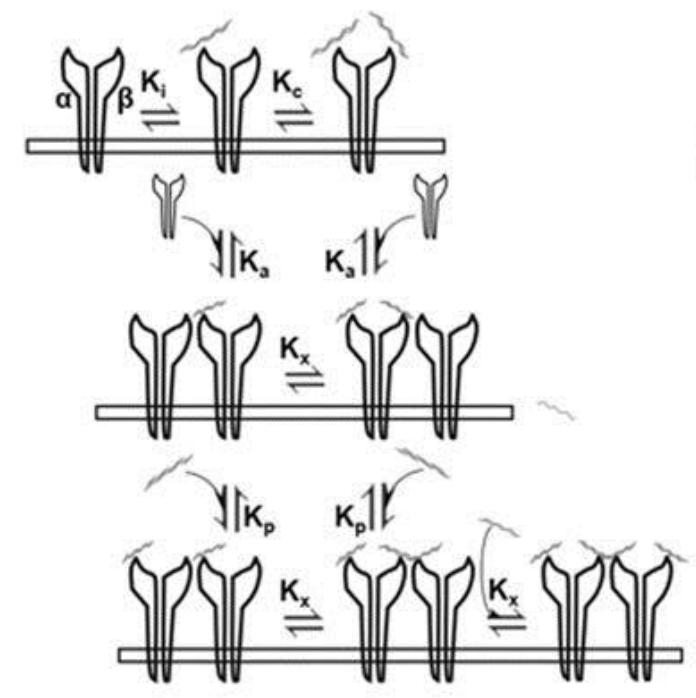

(b)

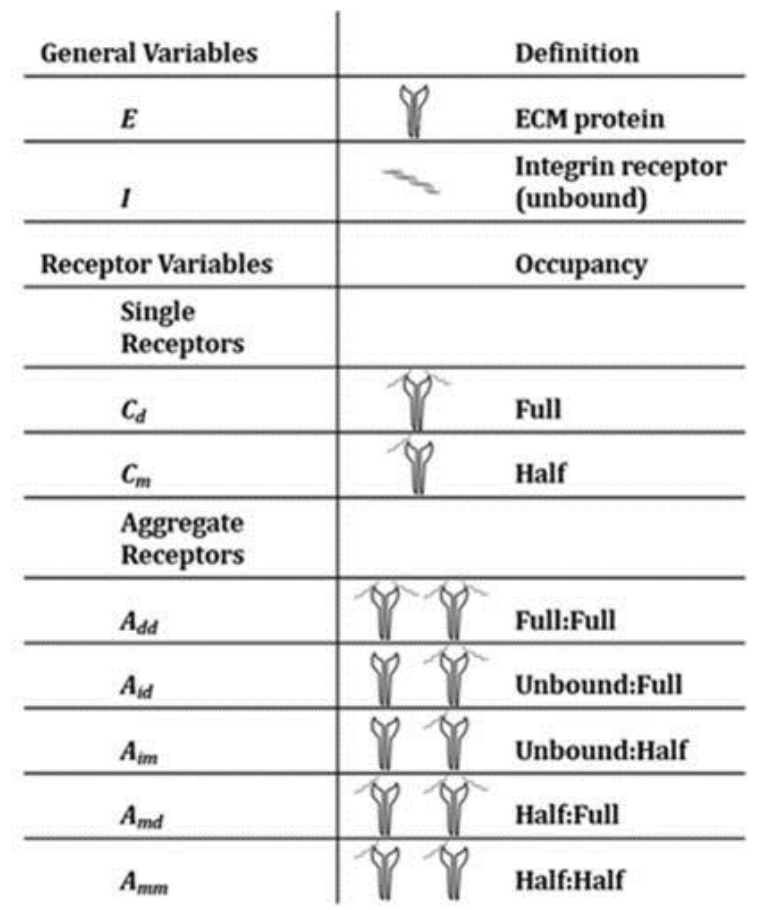


These relationships are represented such that, $k_{1}$ is the first-order association rate constant and $k_{2}$ is the dissociation constant for singly occupied receptors $\left(C_{m}\right) . C_{d}$ indicates a fully occupied integrin receptor with two bound ECM ligands, and $k_{3}$ and $k_{4}$ define the rate constants for association and dissociation, respectively, of the doubly-bound integrin receptor. Differential equations for this model are:

$$
\begin{aligned}
& \frac{d I}{d t}=k_{2} C_{m}-k_{1} I E, \\
& \frac{d E}{d t}=k_{2} C_{m}-k_{1} I E+k_{4} C_{d}-k_{3} C_{m} E, \\
& \frac{d C_{m}}{d t}=k_{1} I E-k_{2} C_{m}+k_{4} C_{d}-k_{3} C_{m} E, \\
& \frac{d C_{d}}{d t}=k_{3} C_{m} E-k_{4} C_{d},
\end{aligned}
$$

The scheme for receptor aggregation and ligand binding is shown in Fig 4. In the model of receptor aggregation, we utilized the same scheme as Wanant et al. [119], wherein receptors pair in a manner such that either singly- or doublybound receptors can aggregate only with an unbound receptor with the aggregation equilibrium constant $\mathrm{K}_{\mathrm{A}}\left(\right.$ where $\left.\mathrm{K}_{\mathrm{A}}=k_{5} / k_{6}\right)$, and disaggregation equilibrium constant $\mathrm{K}_{\mathrm{A}^{\prime}}\left(K_{\mathrm{A}^{\prime}}=k_{6} / k_{5}\right)$. Binding constants for an additional ECM ligand binding to the unbound portion of an aggregate pair are the same regardless of whether the bound portion has one or two ligands, where the equilibrium association constant is $\mathrm{K}_{\mathrm{c}}=k_{9} / k_{10}$. The equilibrium constant for adding a second ECM ligand to a singly-bound receptor in any pair-configuration is 
$\mathrm{K}_{\mathrm{F}}=k_{7} / k_{8}$. The differential equations describing receptor aggregation are listed below:

$$
\begin{aligned}
& \frac{d I}{d t}=k_{2} C_{m}-k_{1} I E+k_{6}\left(A_{i m}+A_{i d}\right)-k_{5} I\left(C_{m}+C_{d}\right), \\
& \frac{d E}{d t}=k_{2} C_{m}-k_{1} I E+k_{4} C_{d}-k_{3} C_{m} E+k_{10}\left(A_{m m}+A_{m d}\right)-k_{9} E\left(A_{i m}+A_{i d}\right)+ \\
& k_{8}\left(A_{i d}+A_{m d}+A_{d d}\right)-k_{7} E\left(A_{i m}+A_{m m}+A_{m d}\right), \\
& \frac{d C_{m}}{d t}=k_{1} I E-k_{2} C_{m}+k_{4} C_{d}-k_{3} C_{m} E+k_{6} A_{i m}-k_{5} I C_{m}, \\
& \frac{d C_{d}}{d t}=k_{3} C_{m} E-k_{4} C_{d}+k_{6} A_{i d}-k_{5} I C_{d} \\
& \frac{d A_{i m}}{d t}=k_{5} I C_{m}-k_{6} A_{i m}+k_{10} A_{m m}-k_{9} E A_{i m}+k_{8} A_{i d}-k_{7} E A_{i m}, \\
& \frac{d A_{i d}}{d t}=k_{5} I C_{d}-k_{6} A_{i d}+k_{10} A_{m d}-k_{9} E A_{i d}+k_{7} E A_{i m}-k_{8} A_{i d}, \\
& \frac{d A_{m m}}{d t}=k_{9} E A_{i m}-k_{10} A_{m m}+k_{8} A_{m d}-k_{7} E A_{m m}, \\
& \frac{d A_{m d}}{d t}=k_{9} E A_{i d}-k_{10} A_{m d}+k_{8} E\left(A_{d d}-A_{m d}\right)+k_{7} E\left(A_{m m}-A_{m d}\right), \\
& \frac{d A_{d d}}{d t}=k_{7} E A_{m d}-k_{8} A_{d d},
\end{aligned}
$$

where $A_{i m}$ indicates an aggregate pair comprised of one unbound integrin receptor coupled with a singly-bound receptor; $A_{\text {id }}$ is the same combination, except featuring a doubly-bound receptor. A pair with two singly-bound receptors is defined as $A_{\mathrm{mm}}$, with two doubly-bound receptors is $A_{\mathrm{dd}}$, and $A_{\mathrm{md}}$ indicates a singly-bound receptor paired with a doubly-bound one (Fig 4). 
The affinity of integrin receptors for ECM proteins fibronectin and laminin are generally in the micromolar range, and the $\mathrm{K}_{d}$ measured for ECM:integrin and in particular, fibronectin binding, ranges between approximately $10^{-7}-10^{-6} \mathrm{M}[120]$; Takagi et al. report nanomolar $\mathrm{K}_{d}$ values for fibronectin binding [121]. Mallet et al. utilized a $\mathrm{Kd}_{\mathrm{d}}$ of $2 \times 10^{-4} \mathrm{M}$ for tethered RGD peptides in their model of integrin binding [116].

5. Simulations

Kinetic simulations were performed as described in Chapter II, Section D.1.

C. Results

1. Animal model phenotype confirmed for fibrotic injury.

As expected, 4 weeks of $\mathrm{CCl}_{4}$ exposure caused robust fibrotic scarring of the liver in our mouse model. The resultant phenotype of injury and fibrosis has been previously described to include degradation of basement membrane-like ECM and replacement with fibrillar collagens and other integrin ligands (Scheme 3.3) [12]. The canonical change in ECM content during hepatic fibrosis is an increase in collagen 1 deposition. However, as has been described by others $[122,123]$, several other proteins increase in response to $\mathrm{CCl}_{4}$-induced fibrosis. 


\section{Scheme 3.3. Schematic of aberrant ECM accumulation following}

$\mathrm{CCl}_{4}$ injury.

Key extracellular matrix proteins (ECMPs) and cognate integrin receptors in $\mathrm{CCl}_{4}$ exposure mouse model of fibrosis. The phenomena include quiescent hepatic stellate cell (HSC) activation and their subsequent differentiation into myofibroblasts after which growth factor-induced proliferation leads to the aberrant ECM deposition that characterizes cirrhotic liver fibrosis. The chronic inflammatory response involves impaired matrix degradation which further contributes to dyshomeostasis of ECM proteins, and therefore tissue structure and errant signal transduction. Following exposure to $\mathrm{CCl}_{4}$, damaged hepatocytes release cellular and membrane components, leading to recruitment of neutrophils and Kupffer cells. Profibrogenic and proinflammatory cytokines, reactive oxygen species (ROS), and proteases are released from resident immune cells, leading to stimulation and activation of quiescent HSCs, inducing their differentiation to myofibroblasts. Proliferation of activated myofibroblasts in response to fibrogenic factors results in excessive ECM deposition, leading to fibrotic scarring and end-stage liver disease. Integrin mediators known to be active in fibrotic pathology include $\beta 1, \alpha 1, \alpha 5$, and $\alpha 6$ on hepatocytes, which correlate clinically with stage of fibrosis. $\alpha v \beta 3$ integrin signaling from HSCs/myofibroblasts is involved with regulating ECM-fibrolytic matrix metalloproteinases. De novo a8 $\beta 1$ expression in activated HSCs occurs in response to $\mathrm{CCl}_{4}$ injury; likewise, $\alpha 1, \alpha 2$, and $\alpha 5$ on $\mathrm{HSCs}$ is indicative of activation, enhancing attachment to basement membrane proteins [110]. Feed 
forward mechanism results from the fibrillar ECM itself enhancing HSC activation, implicating integrins $\alpha_{1} \beta_{1}, \alpha_{2} \beta_{1}$, and $\alpha_{v} \beta_{1}[40]$. 


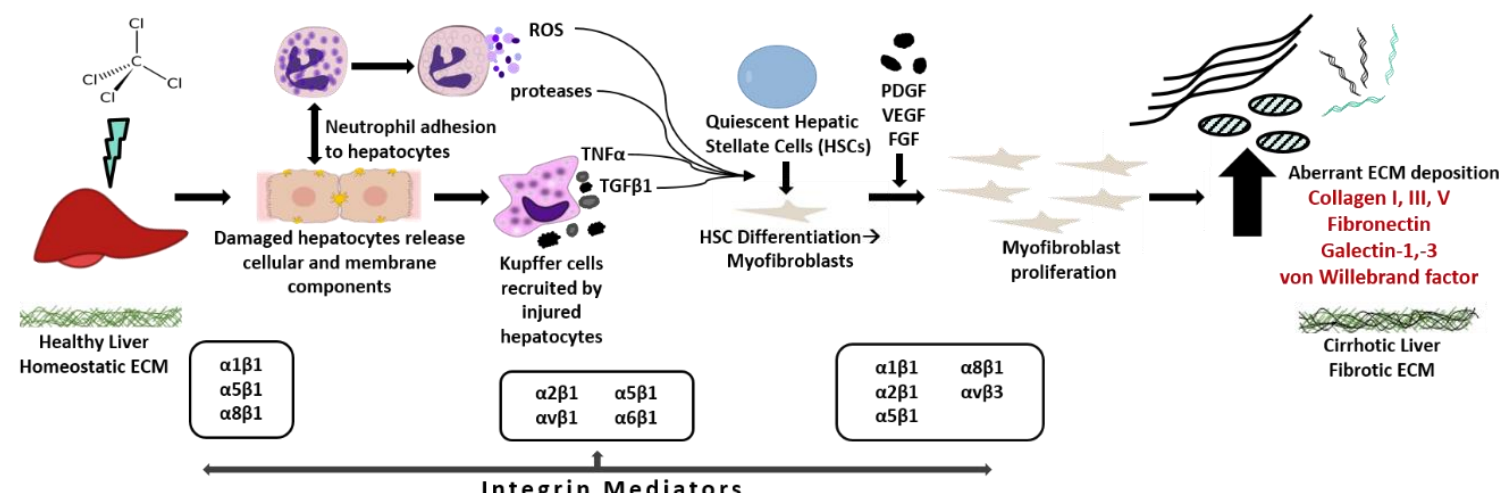


2. Proteomic data-ECM proteins.

Analysis of the proteomic data (Table 3.1 and Figure 3.1) revealed ECM protein expression profiles, and a simple conversion for relating quantitative emPAI values to protein mass was employed as a proteomic ruler to estimate protein concentration under homeostatic and experimental treatment conditions [124]. We converted protein spectra using the exponentially modified protein abundance index (emPAl) quantitative method [125]. Weighting the values with the concentration of extraction fractions, we estimated a relative protein concentration for ECM components. The composition of the liver ECM as quantitated via proteomic analysis has influence on integrin expression of cells that haptotax towards ECM protein gradients, and also provides the pool of available ligands for subsequent binding. 
Table 3.1. Quantitative differential protein expression of liver ECM following 4 weeks of $\mathrm{CCl}_{4}$ exposure

\begin{tabular}{|c|c|c|c|c|c|}
\hline \multirow[t]{2}{*}{ Protein } & \multirow[t]{2}{*}{$\begin{array}{l}\text { GO } \\
\text { Accession }\end{array}$} & \multirow[t]{2}{*}{$\begin{array}{l}\text { MW } \\
\text { (kDa) }\end{array}$} & \multicolumn{2}{|c|}{$\begin{array}{l}\text { Protein Abundance } \\
(\mu \mathrm{M})\end{array}$} & \multirow[t]{2}{*}{$\begin{array}{l}\log _{2} \\
\text { FC }\end{array}$} \\
\hline & & & Con & $\mathrm{CCl}_{4}$ & \\
\hline Col 1a1 & Co1A1 & 138 & 3.60 & 6.42 & 0.83 \\
\hline Col 1a2 & $\mathrm{CO} 1 \mathrm{~A} 2$ & 130 & 2.55 & 4.25 & 0.74 \\
\hline Col 3a1 & CO3A1 & 139 & 0.75 & 1.64 & 1.13 \\
\hline Col 4a1 & CO4A1 & 161 & 0.20 & 0.23 & -0.24 \\
\hline Col 4a2 & $\mathrm{CO} 4 \mathrm{~A} 2$ & 167 & 0.46 & 0.28 & -0.71 \\
\hline Col 5a1 & CO5A1 & 184 & 0 & 0.05 & 11.80 \\
\hline Col 5a3 & Q9JLI2 & 172 & 0 & 0.06 & 11.90 \\
\hline Col 18a1 & E9QPX1 & 182 & 0.07 & 0 & -11.81 \\
\hline Dermatopontin & DERM & 24 & 0 & 7.11 & 16.06 \\
\hline Fibronectin & FINC & 273 & 0.18 & 0.47 & 1.40 \\
\hline Fibrinogen $\beta$ chain & FIBB & 55 & 3.13 & 0.56 & -2.48 \\
\hline Fibrinogen $y$ chain & FIBG & 49 & 5.94 & 1.94 & -0.45 \\
\hline Galectin-1 & LEG1 & 15 & 8.45 & 21.18 & 1.33 \\
\hline Galectin-3 & LG3BP & 64 & 0 & 0.63 & 13.97 \\
\hline $\begin{array}{l}\text { von Willebrand factor } \\
\text { A }\end{array}$ & VMA5A & 87 & 0.33 & 0.84 & 1.33 \\
\hline
\end{tabular}

Table 3.1. Proteomic data for integrin-binding ECM proteins of interest (full dataset not shown). Zeroes were set to 0.00001 for calculation of $\log _{2}$ fold change. The exponentially modified protein abundance index (emPAI) was used for estimation of absolute protein abundance [125] and to approximate protein concentration of relevant integrin-binding proteins. Multidimensional protein 
identification technology (MudPIT) was used to artificially recombine fraction data from Mascot and SequestHT searches and produce quantitation that relates total protein signal in each treatment group $[126,127]$. To normalize for tissue fractionation, the dimensionless emPAl score was weighted with the concentration loaded for each fraction, i.e., $0.25 \mu \mathrm{g} / \mu \mathrm{L}$, to calculate relative protein concentration as initial parameter values for the simulations. 
Figure 3.1. Qualitative Venn diagram of proteomic data.

Differentially expressed proteins of interest for evaluation of ECMP-integrin bindings include collagens, fibrillar proteins, glycoproteins and proteoglycans. Of seven proteins uniquely expressed in the $\mathrm{CCl}_{4}$ experimental model, one ECMP protein, Galectin-3, was identified. One protein was unique to the control, and 90 were differentially expressed. ECMPs used for these simulations are listed in Table 3.1. 


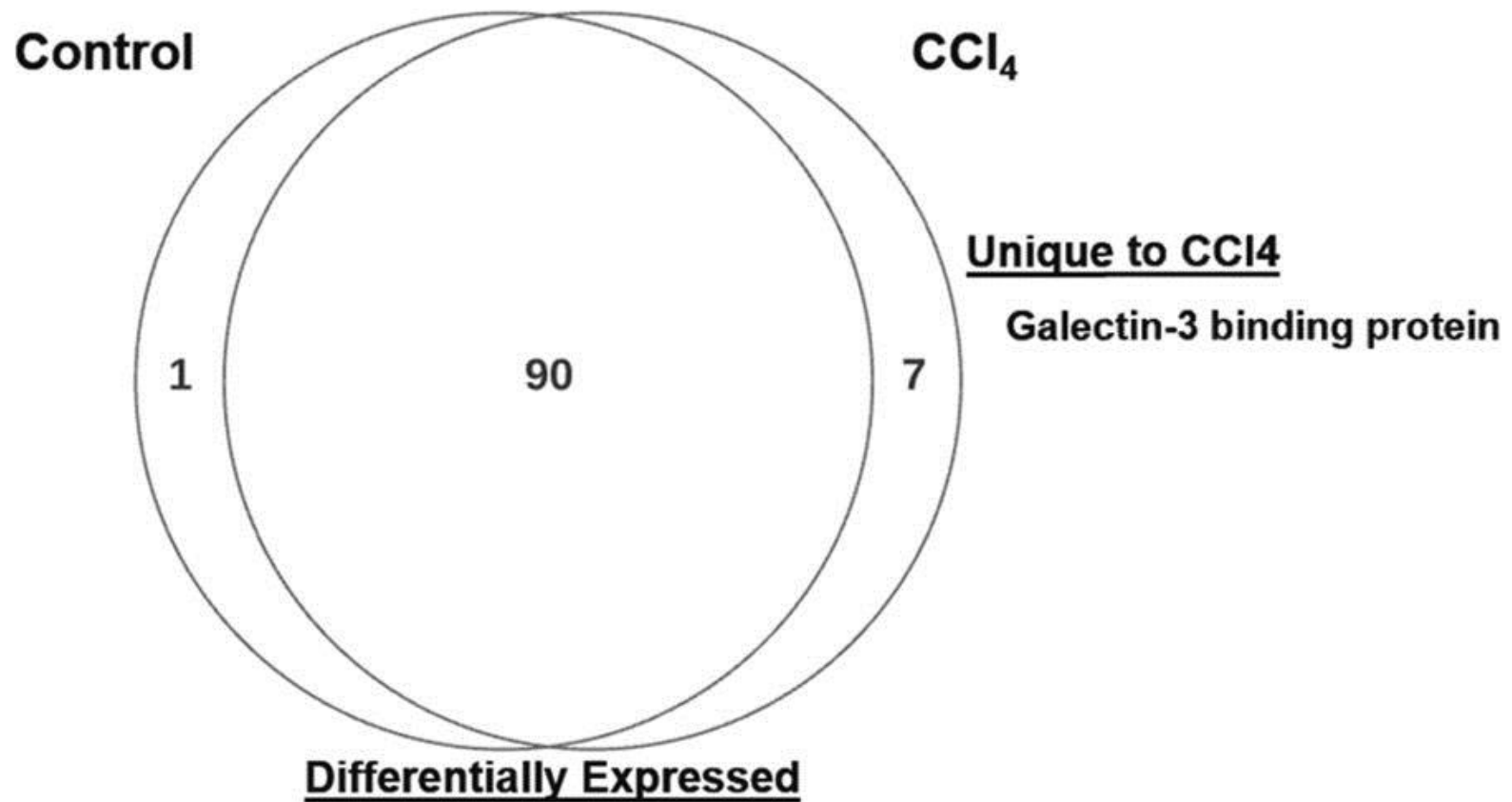

Collagens

Collagen I

Collagen III

Collagen IV

Collagen V

Collagen XVIII
Glycoproteins \& Proteoglycans

Fibronectin

Fibrinogen

Galectin-1

von Willebrand factor $A$ 
Qualitatively, the majority of proteins identified were found in both the control and treatment groups, with seven proteins uniquely expressed in the $\mathrm{CCl}_{4}$ group and only one unique to the control group (Figure 3.1). Collagens, glycoproteins and proteoglycans identified via proteomic analysis as ECM substrate were quantified and their relative concentration was determined (Table 3.1). Beta-1 and Beta-3 integrins selected for simulations reflect those involved in hepatic events that relate to $\mathrm{CCl}_{4}$ fibrosis. Integrin-ECM binding microrates have been determined for various cell types and conditions.

Proteomic results were previously validated to confirm relative abundance of identified proteins qualitatively and quantitatively [45]. In particular, the amounts and distribution of collagens in the treatment group relative to the control were verified. Here, the presence of trace amounts of Col $\mathrm{V}$ in the $\mathrm{CCl}_{4}$ treatment group was validated to explore whether changes on the nanomolar scale would have pathological consequence. (Figure 3.2). 
Figure 3.2. Immunofluorescent staining of hepatic cryosections.

OCT sections were probed with Col V primary antibody and resolved with Alexa 488-tagged secondary antibody. The $\mathrm{CCl}_{4}$ treatment group shows a marked increase in Col V staining relative to the control. 
Control

$\mathrm{CCl}_{4}$

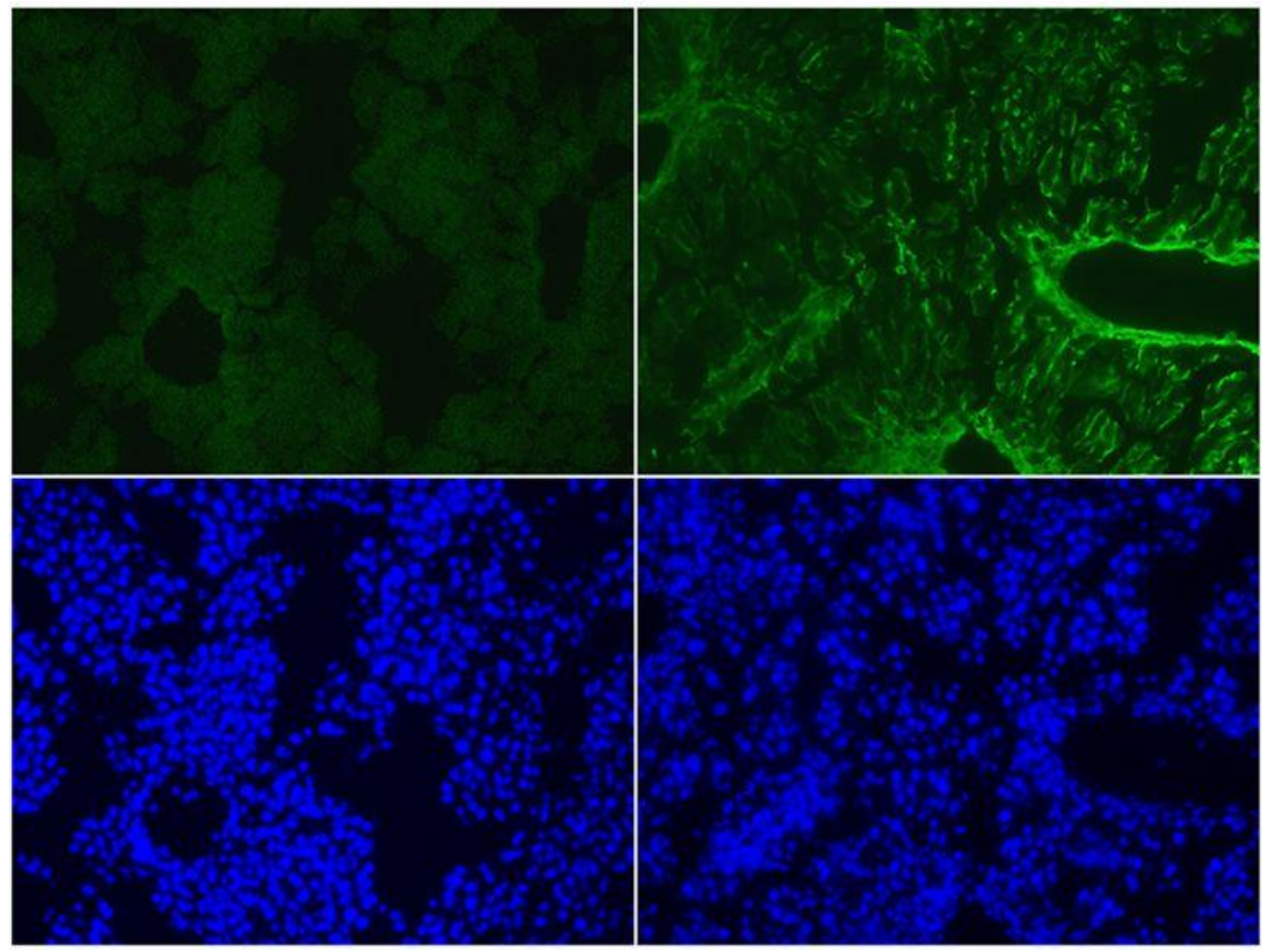

Col V

Dapi 


\section{Simulations.}

The goal herein was to establish a computational framework using proteomic data for binding species to enable evaluation of potential integrin binding. The model of integrin receptor binding kinetics reported here was developed with the consideration of sequential binding of subunits. In silico simulations were parameterized using rate constants that correlate with published literature on binding, or otherwise estimated. The rates are listed in Tables 3.2 and 3.3. 
Table 3.2. Binding rate parameters

\begin{tabular}{|c|c|c|c|c|c|c|c|c|c|}
\hline \multirow[t]{3}{*}{$\begin{array}{l}\text { Integrin } \\
\text { Species }\end{array}$} & \multirow[t]{3}{*}{$\begin{array}{l}\text { Initial } \\
\text { Value } \\
\text { (nM) }\end{array}$} & \multicolumn{8}{|c|}{$\begin{array}{l}\text { Binding Kinetic Parameters } \\
\left(\mathbf{K}_{\text {on }}\left[\mathbf{s}^{-1} \mathbf{M}^{-1}\right] ; K_{\text {off }}\left[\mathbf{s}^{-1}\right]\right)\end{array}$} \\
\hline & & \multicolumn{2}{|c|}{ Col I } & \multicolumn{2}{|c|}{ Col IV } & \multicolumn{2}{|c|}{ Fibronectin } & \multicolumn{2}{|c|}{$\begin{array}{l}\text { von Willebrand } \\
\text { factor } A\end{array}$} \\
\hline & & Kon & Koff & Kon & Koff & Kon & Koff & Kon & Koff \\
\hline$\alpha 1 \beta 1$ & 0.0001 & $5.6 \times 10^{4}$ & $1.3 \times 10^{-3}$ & $8.0 \times 10^{5}$ & $5.0 \times 10^{-3}$ & & & & \\
\hline$\alpha V \beta 3$ & 0.05 & & & & & $1.6 \times 10^{8}$ & $3.5 \times 10^{-1}$ & $1.6 \times 10^{4}$ & $2.3 \times 10^{-2}$ \\
\hline
\end{tabular}

Table 3.2. Initial conditions and reaction rate parameters of kinetic models - collagens, glycoproteins and proteoglycans. Rates for binding ECM components relevant to the $\mathrm{CCl}_{4}$ model are delineated per cognate integrin receptor. From published literature initial values of integrin receptor species are derived and given in nanomolar; similarly, binding rate parameters are given in $\mathrm{s}^{-1} \mathrm{M}^{-1}$ for $\mathrm{K}_{\text {on }}$ and $\mathrm{s}^{-1}$ for $\mathrm{k}_{\text {off; }}$ [121, 128-134]. 
Table 3.3. Equilibrium binding constants for receptor aggregation model.

\begin{tabular}{|c|c|c|c|}
\hline $\begin{array}{l}K_{d} \\
(n M)\end{array}$ & Reaction & $\begin{array}{l}\text { Microrates } \\
\text { (on; } \mathrm{nM}^{-1} \mathbf{s}^{-1} / \mathrm{off} ; \\
\mathrm{s}^{-1} \text { ) }\end{array}$ & Manipulations \\
\hline $\mathbf{K}_{\mathbf{i}}$ & $\begin{array}{l}\text { Integrin complex } \\
\text { formation }\end{array}$ & $k_{2} / k_{1}$ & $\begin{array}{l}\mathrm{K}_{\mathrm{p}}>\mathrm{K}_{\mathrm{i}} \\
\text { increase in ligand affinity } \\
\text { after aggregation }\end{array}$ \\
\hline $\mathbf{K}_{\mathbf{c}}$ & $\begin{array}{l}\text { Filling divalent } \\
\text { unpaired receptor }\end{array}$ & $k_{4} / k_{3}$ & $\begin{array}{l}\mathrm{K}_{\mathrm{c}}=0.01 \mathrm{~K}_{\mathrm{i}} \\
\text { decreased unpaired } \\
\text { receptor } \mathrm{K}_{\text {eq }} \text { for binding } \\
2^{\text {nd }} \text { ligand }\end{array}$ \\
\hline $\mathrm{K}_{\mathbf{a}}$ & $\begin{array}{l}\text { Empty receptor } \\
\text { pairing with bound } \\
\text { receptor }\end{array}$ & $k_{6} / k_{5}$ & $\begin{array}{l}\mathrm{K}_{\mathrm{a}}>0 \\
\text { aggregation constant } \\
\text { drives positive } \\
\text { cooperativity }\end{array}$ \\
\hline$K_{p}$ & $\begin{array}{l}\text { Population of empty } \\
\text { paired receptors }\end{array}$ & $k_{10} / k_{9}$ & $\begin{array}{l}K_{p}>K_{i} \\
\text { increase in ligand affinity } \\
\text { after aggregation } \\
K_{p}=100 K_{x} \\
\text { decreased aggregate } \\
\text { receptor Keq for binding } \\
2^{\text {nd }} \text { ligand }\end{array}$ \\
\hline$K_{\mathbf{x}}$ & Receptor saturation & $k_{8} / k_{7}$ & $\begin{array}{l}K_{p}=100 K_{x} \\
\text { decreased aggregate } \\
\text { receptor Keq for binding } \\
2^{\text {nd }} \text { ligand }\end{array}$ \\
\hline
\end{tabular}

Table 3.3. Microrate parameters are derived from published values and set to implement positive cooperativity for sequential ligand binding and receptor aggregation. The rates for integrin complex formation $\left(\mathrm{K}_{\mathrm{i}}\right)$ are set to simulate an increase in ligand affinity post-aggregation. Populating an empty unpaired receptor is set with a hundredth fold decrease in Keq for binding the second ligand. The aggregation equilibrium constant is set at ten times the equilibrium constant for initial complex formation to allow for aggregation to drive positive cooperativity. The population of empty paired receptors dictates an increase in 
ligand affinity after aggregation and is set to decrease aggregate receptor $\mathrm{K}_{\mathrm{eq}}$ for binding second ligand for receptor saturation. These parameters are adapted from Wanant et al. [119], and applied here to simulate positive cooperativity in receptor aggregation pairing so that the model can be initialized and implemented with proteomic data to evaluate binding profiles. 
The simulations were initialized using binding constants from published literature; where values were not available, parameters were estimated accordingly (see Methods). Collagen fragments for collagen I and IV were plotted together (Figure 3.3) and assumed to have the same rates of binding for the purposes of these experiments. For the other fragmented protein, fibrinogen, only the gamma subunit was considered due to the binding motif located within this fragment $[135,136]$. The binding microrates were set to recapitulate positive cooperativity in divalent receptor saturation and in receptor aggregation pairs, as stipulated in Wanant et al. [119], wherein the aggregation equilibrium constant, $\mathrm{K}_{\mathrm{a}}$, drove cooperativity in the aggregate model (Table 3.3).

The simulation graphs in Figure 3.3 show left-shifted curves with increased ECM ligand abundance, indicating increased affinity and avidity for ligand. This is reflected in both the curves for fully-occupied divalent receptors $\left(C_{d}\right)$ and for fully-occupied aggregate receptor pairs $\left(A_{d d}\right)$. Steady-state values (SS) and time to steady-state were recorded for each simulation (Table 3.4). 
Table 3.4. Steady-state values for simulations of binding.

\begin{tabular}{|c|c|c|}
\hline ECMP, Treatment & Integrin Receptor & Steady-state $\left[C_{d}\right]$ \\
\hline $\mathrm{vWF}, \mathrm{CCl}_{4}$ & $\alpha v \beta 3$ & $2.19 \times 10^{5}$ \\
\hline ECMP, Treatment & Integrin Receptor & Steady-state $\left[A_{d d}\right]$ \\
\hline $\mathrm{VWF}, \mathrm{CCl}_{4}$ & $\alpha v \beta 3$ & $5.70 \times 10^{4}$ \\
\hline ECMP, Treatment & Integrin Receptor & $\begin{array}{l}\text { Time to Steady-state } \\
\left(\mathrm{sec} ; C_{d}\right)\end{array}$ \\
\hline Col $1 \alpha 1, \mathrm{CCl}_{4}$ & $\alpha 1 \beta 1$ & $4.02 \times 10^{1}$ \\
\hline Col 1a1, $\mathrm{CCl}_{4}$ & $\alpha 1 \beta 1$ & $4.67 \times 10^{1}$ \\
\hline Col 4a2, Control & $\alpha 1 \beta 1$ & $5.23 \times 10^{1}$ \\
\hline $\mathrm{Col} 1 \alpha 2, \mathrm{CCl}_{4}$ & $\alpha 1 \beta 1$ & $6.88 \times 10^{1}$ \\
\hline $\mathrm{Col} 4 \alpha 2, \mathrm{CCl}_{4}$ & $\alpha 1 \beta 1$ & $7.94 \times 10^{1}$ \\
\hline Col 1a1, Control & $\alpha 1 \beta 1$ & $8.49 \times 10^{1}$ \\
\hline ECMP, Treatment & Integrin Receptor & $\begin{array}{l}\text { Time to Steady-state } \\
\left(\mathrm{sec} ; A_{d d}\right)\end{array}$ \\
\hline $\mathrm{Col} 1 \alpha 1, \mathrm{CCl}_{4}$ & $\alpha 1 \beta 1$ & $1.00 \times 10^{2}$ \\
\hline Col 1a2, $\mathrm{CCl}_{4}$ & $\alpha 1 \beta 1$ & $1.00 \times 10^{2}$ \\
\hline
\end{tabular}

Table 3.4. Steady-state (SS) and time to SS values for a fully-occupied single divalent receptor $\left(C_{d}\right)$ and saturated aggregated receptor pairs $\left(A_{d d}\right)$. Top values for each parameter are given. 
Figure 3.3. Kinetic simulations data.

Model was initialized using ligand concentrations from proteomic analysis (Table 3.1) and kinetic rates listed in Table 3.2. The ECM:integrin binding pairs fibronectin: $\alpha v \beta 3$, von Willebrand factor: $\alpha v \beta 3$, and collagen I: $\alpha 1 \beta 1$ are shown, with binding curves and percent occupancy for fully occupied single divalent receptors $\left(C_{d}\right)$ and aggregated receptor pairs $\left(A_{d d}\right)$. 

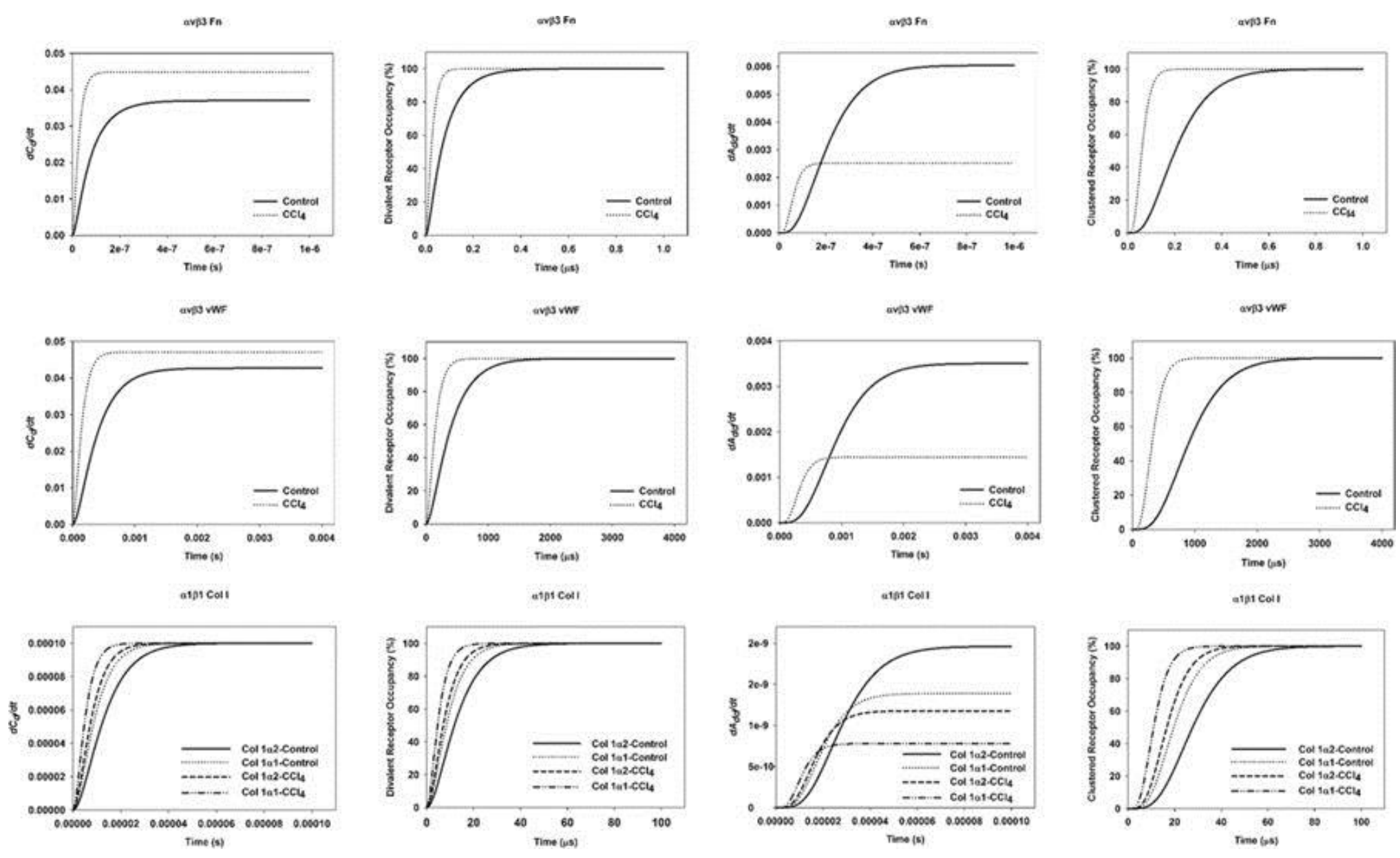
From these simulation data it is clear that upregulated ECMPs reached steady state values in shorter time, and that aggregation of receptors produced positive cooperativity. Considering the single divalent receptor, $C_{d}$, the ECM:integrin binding pairs that had the highest steady-state values include both collagen 1 and fibrinogen $y$ chain in association with the $\alpha v \beta 3$ integrin receptor. The combinations with the shortest time to SS were collagen 1 binding $\alpha v \beta 3$ or $\alpha 1 \beta 1$ receptors. For the aggregated receptor pairs, $A_{d d}$, the pairs with the highest SS values include von Willebrand factor, fibrinogen y chain, and collagen 1 binding $\alpha v \beta 3$, as well as fibronectin binding $\alpha 5 \beta 1$. The ECM:integrin pairings with the shortest time to SS for $A_{d d}$ pairs were collagen 1 binding $\alpha \vee \beta 3$ and $\alpha 1 \beta 1$, and fibronectin binding a5 31 . In nearly all cases, the $\mathrm{CCl}_{4}$ model ECM showed faster rise to SS compared to the control ECM; however, fibrinogen y chain and Col $4 \alpha 2$ behaved in an opposite manner, owing to the fact that $\mathrm{CCl}_{4}$ actually downregulated these ECMPs in our dataset.

\section{Discussion}

Integrin binding to ECM is a vital mechanism for cell migration, invasion, proliferation, and signal transduction between cells and their microenvironment. Diseases of chronic inflammation and injury, including fibroses and cancer, involve persistent dysregulation of ECM-integrin processes and induce remodeling of the ECM. In addition to their intrinsic utility in cellular processes, association between immune cells and the ECM is regulated via the $\beta 1 \& \beta 3$ integrin receptor subfamilies [137]. Several mathematical models of integrin 
binding have been reported with outputs relating to spatial clustering and signal transduction, liver fibrosis, and cell migration $[116,118,138,139]$. These models recapitulated certain aspects of integrin interactions; however, these previous studies typically modeled only one ligand, mainly fibronectin or collagen, and utilized generic cognate receptor.

In this study, the relative abundance of ECM components that are canonical substrates of integrin receptors was developed for the proposed modeling framework based on experimentally-obtained liver ECM data. With binding parameters from published literature, an integrin binding pattern was established for each integrin involved in hepatic processes that are involved in fibrosis. The model from Wanant et al. was adapted to implement the basic model for divalent binding [119]. Specifically, this model aptly describes initial integrin binding leads to a conformational switch of the receptor complex from low- to high-affinity. A model of receptor aggregation, which can describe integrin clustering upon attachment to ECM via adhesions [16], was also implemented. This study simulates how each integrin binds with cognate ECM ligands and incorporates the varying affinities that drive this interaction. From these calculations, the kinetic indices of each integrin for each of its binding partners were determined separately. The impact of changes to the ECM (e.g., in response to $\mathrm{CCl}_{4}$-induced fibrosis) on integrin binding was modeled by calibrating the substrate concentration based on the proteomic analyses. The extracellular matrix proteome was consistent with the known disease phenotype of the mouse model, with upregulation of specific ECMPs involved in fulminant fibrosis. The 
computational results show that in simulations using these ECMPs as substrate for key integrin receptors, interactions involving profibrotic integrins were predominant.

The $\mathrm{CCl}_{4}$ mouse model of liver fibrosis was chosen for development and validation of the in silico model due to its robustly characterized pathology and ECM/integrin phenotype (Scheme 3.3). This model is imperfect in its recapitulation of human liver fibrosis, but it is the current research standard and therefore has well-defined pathology and changes to the ECM [140]. Using proteomic data from $\mathrm{CCl}_{4}$-exposed mouse livers, integrin binding can be explored within the context of fulminant fibrosis. Collagen type la1, type III and type IV are excessively deposited due to activated hepatic stellate cells (HSCs) in response to myofibroblastic transformation induced by activated Kupffer cells and damaged hepatocytes $[40,141]$. In agreement with these established phenomena, collagens $\mathrm{I}, \mathrm{III}$, and $\mathrm{V}$ were upregulated in the $\mathrm{CCl}_{4}$ cohort in the current study (Table 3.1). Collagen I is aberrantly produced in this mouse model, and collagen $\mathrm{V}$, a potent nucleating effector for the co-upregulated fibronectin, exhibited a slight increase from trace levels. In contrast, collagen IV and XVIII levels were decreased relative to the control. Interestingly, collagen XVIII was identified at relatively minimal levels in the controls, and absent in the $\mathrm{CCl}_{4}$ treated animals (Table3.1). This is contrary to an expected increase in collagen XVIII following $\mathrm{CCl}_{4}$ treatment [122]. Nevertheless, interactions simulated with this ECMP are still based on experimental proteomic analysis. Integrin receptors were not able to be resolved with this particular method of proteomic analysis, so 
further proteomic analysis of integrin adhesion complexes in culture is a key component of the future directions for this project.

Owing to their involvement in several critical functions that drive homeostasis and dyshomeostasis, integrins have been identified as key druggable targets in several diseases. For example, integrin inhibitors have been evaluated to suppress liver fibrogenesis, disrupt attachment and invasion of cancer cells, and to mediate immune response [71, 142-144]. Regrettably, many of these drugs fail in early trials and rarely make it to the clinics, perhaps due to an incomplete understanding of integrin binding kinetics, which are traditionally based on single-species models and assumptions; indeed, even antibodies and small peptide mimetics with specificities for multiple integrins have limited clinical application $[145,146]$. Though necessary to target multiple integrins to maximize efficacy in vivo, perhaps the missing link is knowing which targeted doses are most effective for each anti-integrin molecule. In attempting to begin to develop a predictive tool for effective dosing, the primary goal of this work was to create a first step to simulate simple receptor aggregation and reproduce positive cooperativity induced by aggregate pairing. The simulations were parameterized to analyze for positive cooperativity of binding in the divalent and aggregation cases. The steady-state values and time to steady-state for each pairing correlated to upregulation of key ECMPs in $\mathrm{CCl}_{4}$ liver injury (Scheme 3.3; Table 3.4). The integrin receptors that predominated simulations of occupancy were consistent with those known to be at play in this disease model (Figure 3.3). 
The proposed framework has been validated using data from a model of fulminant fibrosis; other liver pathologies and how the transitional remodeling of the ECM affects ECM-integrin interactions could be explored. By testing homeostatic conditions against experimental treatment models, this platform could be broadly employed to predict or confirm changes in integrin binding (and by extension, signaling) caused by remodeling of the hepatic ECM in response to insult or injury. Longer term, a more complex stochastic model for concurrent integrin binding building upon the results of this study could be developed that considers competitive binding of multiple species. This would lay the foundation for a more detailed and nuanced analysis of ECM:integrin interactions. 


\section{CHAPTER IV}

\section{ARSENIC ATTENUATES LIPOPOLYSACCHARIDE-STIMULATED POLARIZATION IN MURINE BONE MARROW DERIVED MACROPHAGES}

\section{A. Introduction}

Inorganic arsenic is a ubiquitous element and a natural drinking water contaminant, typically occurring in the environment in its arsenic oxide $\left[\mathrm{As}_{2} \mathrm{O}_{5}\right]$ or trioxide $\left[\mathrm{As}_{2} \mathrm{O}_{3}\right]$ forms $[147,148]$. Owing to its toxic potential to humans, it is a high priority hazardous substance in the United States, where ground water arsenic is elevated in many areas of the country $[149,150]$. Nearly 4,000 community wells in the U.S. have arsenic levels greater than the current recommended maximum contaminant level (MCL) of $10 \mathrm{ppb}$; in fact, there are an increasing number of community ground water wells that registered above the previous MCL of $50 \mathrm{ppb}$. Public water systems in Western states are also implicated, with some reaching levels over 90 ppb [149, 150]. Furthermore, arsenic concentrations in the ppm range have been reported in private artesian wells, which are not regulated by the Safe Drinking Water Act [151].

Chronic exposure to high arsenic concentrations has been directly linked with a myriad of possible health risks, including skin lesions, hypertension, cardiovascular disease, respiratory disease, and malignancies of the skin and 
internal organs [152]. The arsenic concentrations required to cause disease are generally considered higher than those found in the U.S. drinking water supply. The paradigm in environmental research has recently shifted to take into account the impact of not only high environmental or occupational exposures, but also subacute and chronic low grade exposures; in this context, it is critical to evaluate the impact of multiple aspects of environmental factors that may contribute to the composite health risk (i.e., 'exposure biology') [153]. Some studies indicate that moderate to low arsenic exposure may modify disease risk from other, separate etiologies. For example, previous studies from this group indicate that arsenic exposure at concentrations that are not directly hepatotoxic, per se, modifies experimental inflammatory liver damage $[154,155]$. In humans, the risk of developing some diseases (e.g., Type 2 Diabetes Mellitus (T2DM) in obese patients) has been suggested to be increased by low-level arsenic exposure [156]. The risk of arsenic exposure to human disease may therefore be incompletely understood.

It is now well understood that dysregulation of macrophage responses contributes to both malignant [157] and non-malignant diseases such as T2DM [158]. A growing body of evidence indicates that arsenic impacts the innate immune response in both experimental models and in humans [159-163]. The mechanisms by which arsenic potentially alters macrophage function are not completely understood. Previous studies by this group indicated that sub-toxic arsenic exposure enhanced recruitment of proinflammatory macrophages to the mouse liver during experimental non-alcoholic fatty liver disease (NAFLD) [155]. 
The a priori hypothesis was therefore that arsenic would enhance LPS-induced M1 phenotype, contributing to macrophage mediated inflammation.

Previous studies have investigated the impact of arsenic exposure on the alternative activation of terminally differentiated macrophages (e.g., tissue macrophages), using cell cultures such as RAW 264.7 murine macrophages $[164,165]$. Cui et al. showed the effect of M2 polarization following long-term arsenic exposure [166]. However, these in vitro models present a challenge in terms of evaluating stimulation and polarization, as they often have an inherent basal expression of proinflammatory genes. The differentiation of macrophage precursors to their terminally differentiated phenotype is another level of regulation of macrophage function. The impact of arsenic exposure on this level of macrophage regulation has not been tested. In attempt to reduce this potential background effect and derive a more homogeneous, naïve pool of macrophages, BMDM primary cells were utilized for these experiments. The purpose of the current study was therefore to explore the impact of arsenic exposure on the polarization of naïve, uncommitted bone-marrow derived macrophages.

\section{B. Experimental Procedures}

1. Animals and treatments

Male C57BI6/J mice (6 wk) were obtained from the Jackson Laboratory (Bar Harbor, ME). Bone marrow derived macrophages harvested as described in Chapter II, Section A.2. 
2. BMDM cell culture and treatments

Cells isolated and cultured as indicated in Chapter II, Section G.2.

3. BMDM MTT assay

Cell viability assay completed as indicated in Chapter II, Section G.3.

4. Flow cytometry

Cells analyzed via flow cytometry as described in Chapter II, Section G.1.

5. Quantitative RT-PCR

mRNA expression of select genes was detected by quantitative reversetranscriptase polymerase chain reaction (qPCR), as previously described in Chapter II, Section E. PCR primers and probes for TNFa, iNOS, and PAI-1 were designed using Primer 3 (Whitehead Institute for Biomedical Research, Cambridge, MA); those for IL-1 $\beta$ and $18 \mathrm{~S}$ rRNA were purchased from Applied Biosystems (Foster City, CA).

6. Microarray analysis of miRNA miRNA analysis completed as described in Chapter II, Section F.

C. Results

1. Sodium arsenite attenuates LPS-induced polarization of BMDMs, but not IL-4-induced polarization 
To test the effects of sodium arsenite on LPS-induced macrophage polarization, BMDMs were exposed to LPS in the presence/absence of with varying concentrations of sodium arsenite followed by characterization using flow cytometry (Figure 4.1). CD11c surface expression in F480+/CD11b+ BMDMs was measured as an index of M1 macrophage phenotype [167]. Arsenite exposure did not significantly impact cell survival at any concentration below 500 ppb (Figure 4.1A.); all subsequent experiments were therefore performed at these sublethal concentrations. As expected, LPS exposure significantly increased the number of CD11c+ cells (Figure 4.1B and 4.1C). At all concentrations tested (0-100 ppb), sodium arsenite alone did not significantly affect surface expression CD11c in F480+/CD11b+ in BMDMs (Figure 4.1B), however sodium arsenite (100 ppb) significantly attenuated the increase in the number of CD11c+/F480+/CD11b+ cells caused by LPS (100 ng/ml). At all concentrations tested, sodium arsenite also significantly attenuated the increase in MFI caused by LPS exposure, with a maximal effect at $100 \mathrm{ppb}$ (Figure 4.1C; Figure 4.2A-B); this concentration of arsenic significantly attenuated the increase in CD11c MFI caused by 5 log-order concentrations of LPS. Based on these results, all subsequent studies employed $100 \mathrm{ppb}$ sodium arsenite and $100 \mathrm{ng} / \mathrm{ml}$ LPS. In contrast to LPS, which polarizes BMDMs to a proinflammatory (i.e., 'M1') phenotype, other stimuli (e.g., IL-4) polarize these cells into an anti-inflammatory (i.e., 'M2') phenotype. The impact of sodium arsenite (100 ppb) on the polarization of BMDMs by IL-4 was therefore investigated. Specifically, the number of CD206+/ F480+/CD11b+ and CD206+ MFI was determined by flow 
cytometry (Figure 4.1D; Figure 4.2A). As expected, IL-4 dramatically increased the number of $C D 206+/ F 480+/ C D 11 b+B M D M s$, with a maximal effect at 20 $\mathrm{ng} / \mathrm{ml}$. Sodium arsenite did not significantly impact the number (Figure 4.1D) or intensity of CD206 fluorescence alone or at any concentration of IL-4 tested. 
Figure 4.1. Dose optimization for LPS and sodium arsenite to find lowest effective dose.

BMDMs were assessed for cell viability under treatment with LPS and As(III) to find optimized dose for subsequent experimental analyses. (A): Effect on cellular viability measured using MTT assay of varying concentrations of sodium arsenite with or without cotreatment of $100 \mathrm{ng} / \mathrm{mL}$ LPS. (B): CD11c MFI for $\mathrm{F} 4 / 80^{+} / \mathrm{CD} 11 \mathrm{~b}^{+}$cells after flow cytometry analysis of differentiated BMDMs treated with varying concentrations of sodium arsenite for $18 \mathrm{~h}$ in the presence/absence of $100 \mathrm{ng} / \mathrm{mL}$ LPS. (C): Concentration-response curve of LPS treated BMDMs and CD11c MFIs in the presence (open circles) or absence (black circles) of $100 \mathrm{ppb}$ sodium arsenite. (D): Concentration-response curve of IL-4 treated BMDMs and CD206 MFIs in the presence (open circles) or absence (black circles) of $100 \mathrm{ppb}$ sodium arsenite. Values are presented as means \pm S.E.M. ( $\mathrm{n}=3$ to 4 ), a, $P<0.05$, compared to control, $, b, P<0.05$, compared to respective LPS treatment. 
A.
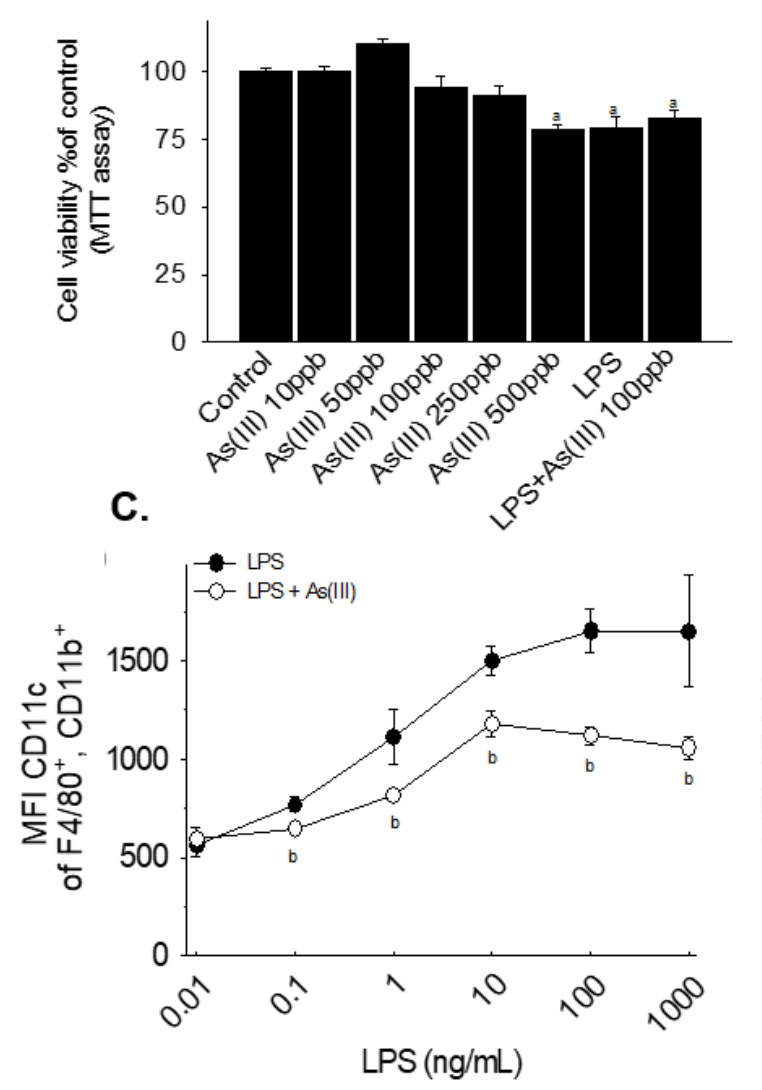

B.
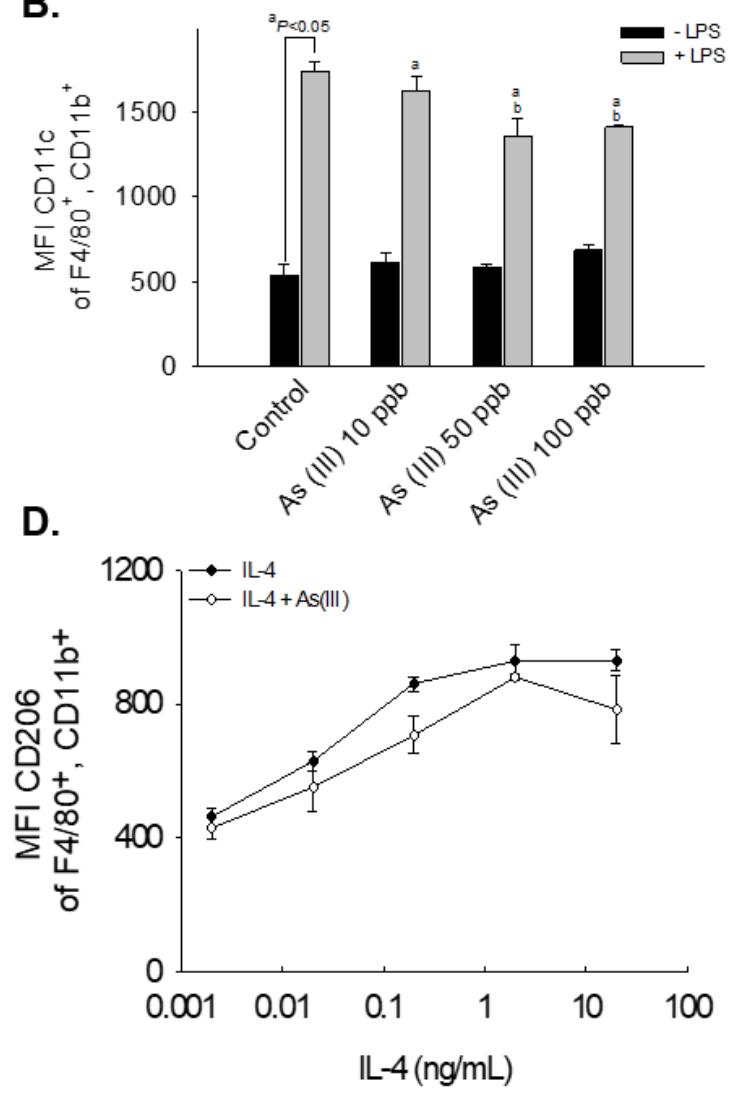
Figure 4.2. Flow cytometric analysis of macrophage polarization.

BMDMs polarized with either LPS (M1) or IL-4 (M2) and treated with As(III) were analyzed via flow cytometry with macrophage cell surface markers relative to each polarization state. Representative of flow cytometry plots are shown; data shown comprises cell numbers in the CD11 $\mathrm{c}^{+}$fraction of $\mathrm{F} 4 / 80^{+} / \mathrm{CD} 11 \mathrm{~b}^{+}$ population. (A): Representative off-set histograms of flow cytometry are shown; left, M1 polarization marker CD11c, right, M2 polarization marker CD206. Representative off-set histograms of flow cytometry are shown. (B): 2D scatterplot for quantification of cell numbers in the CD11 $\mathrm{c}^{+}$fraction of $\mathrm{F} 4 / 80^{+} / \mathrm{CD} 11 \mathrm{~b}^{+}$population. 

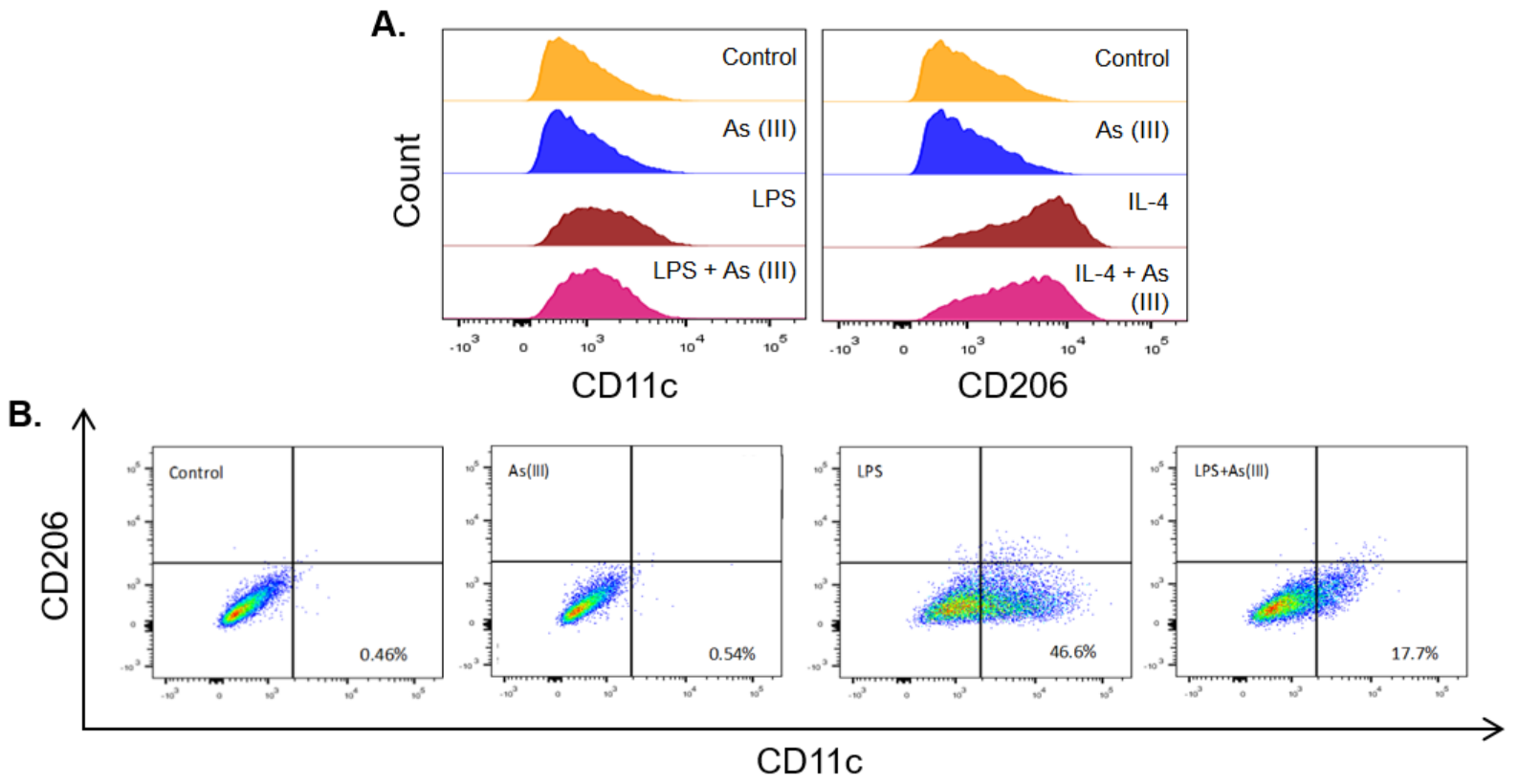
2. Sodium arsenite modifies constitutive and LPS-induced mRNA expression levels of TNF- $\alpha$, IL-1 $\beta$, iNOS, and PAI-1

To further evaluate the impact of sodium arsenite on LPS-induced macrophage polarization, pro-inflammatory gene (TNF- $\alpha, \mathrm{IL}-1 \beta$, iNOS, and PAI1) mRNA levels were determined. For this purpose, BMDMs were treated with $100 \mathrm{ppb}$ As(III), $100 \mathrm{ng} / \mathrm{mL}$ LPS, or LPS+ sodium arsenite for $6 \mathrm{~h}$. This time point was chosen from pilot studies for the maximal expression of mRNA and miRNAs. Sodium arsenite alone did not significantly affect TNF- $\alpha$ mRNA levels, though it significantly decreased IL-1 $\beta$ and iNOS and significantly increased PAI-1 mRNA levels compared to control (Figure 4.3). LPS exposure robustly increased TNF-a, $\mathrm{IL}-1 \beta$, iNOS, and PAI-1 mRNA compared to control as expected for the M1 polarization phenotype (Figure 4.3). Importantly, sodium arsenite significantly attenuated the LPS-mediated induction of TNF- $\alpha$, IL-1 $1 \beta$, iNOS, and PAI-1 mRNA levels compared to LPS alone. 
Figure 4.3. Effect of sodium arsenite on constitutive and LPS-induced mRNA expressions.

Differentiated BMDMs were treated with different concentrations of sodium arsenite for $6 \mathrm{~h}$ in the presence/absence of $100 \mathrm{ng} / \mathrm{mL}$ LPS. Real time RT-PCR for the pro-inflammatory genes TNF $\alpha(A), I L-1 \beta(B)$, iNOS (C) in addition to PAI-1 (D) was performed as described in the Methods section. ${ }^{a,} p<0.05$, compared to control; $b, p<0.05$, compared to respective LPS treatment. Quantitative data are shown as means \pm SEM $(n=3)$. 

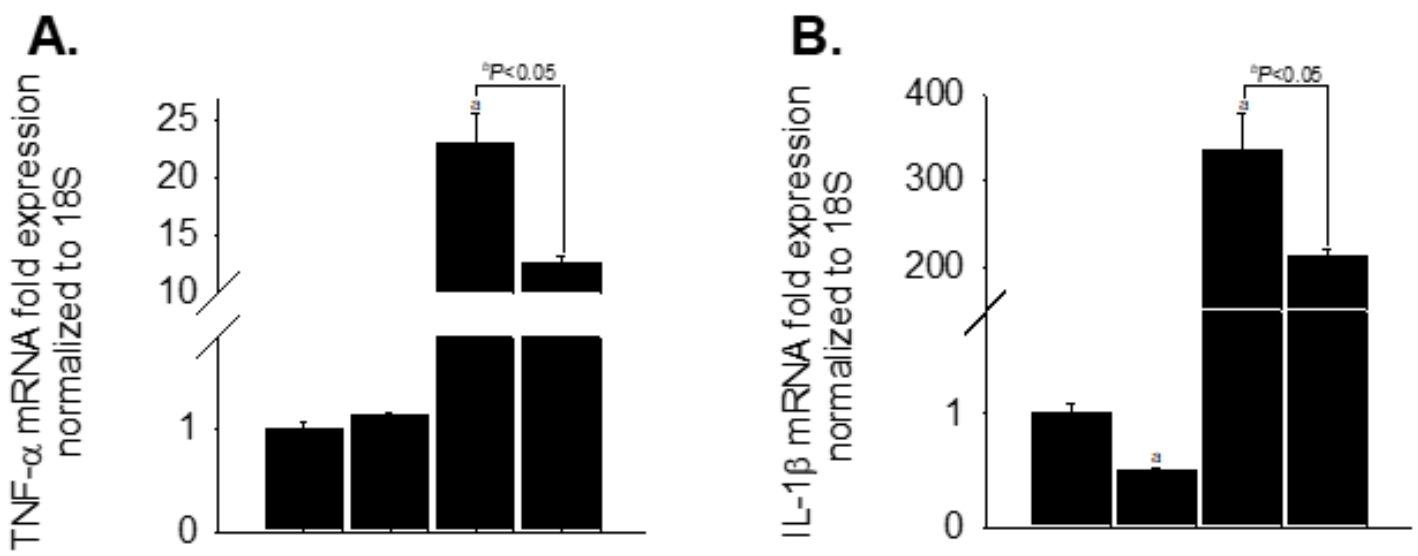

c.

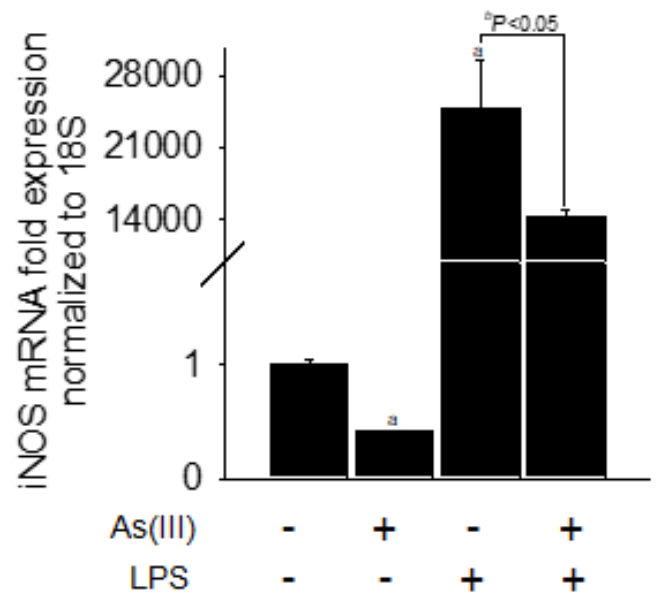

D.

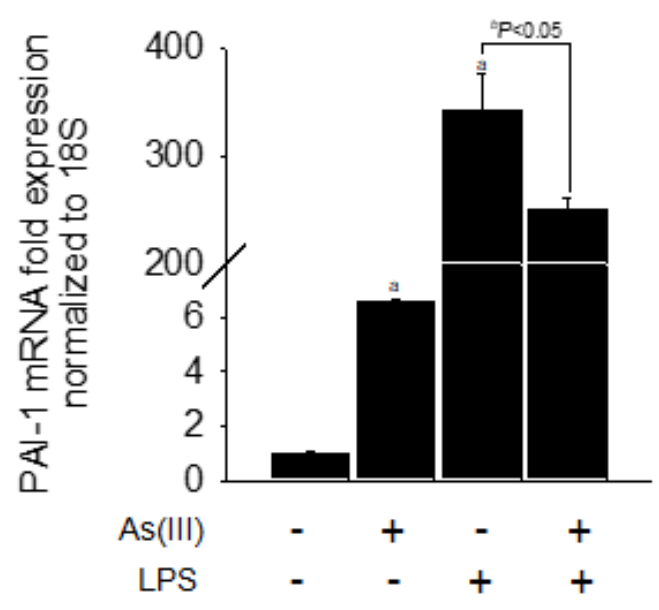


3. Differential miRNA expression profiles of BMDMs treated with As(III), LPS, or LPS+As(III)

Emerging evidence shows that macrophage miRNAs have close relationship with macrophage polarization [168-171]. Furthermore, arsenic exposure has been demonstrated to impact miRNA expression in vitro and in vivo [172]. In order to shed light on BMDMs miRNA profiles, miRNA arrays were performed to identify differentially expressed miRNAs in BMDMs treated with $100 \mathrm{ppb}$ As(III), 100 $\mathrm{ng} / \mathrm{mL}$ LPS, or LPS+As(III). miRNAs identified to be significantly differentially expressed in the three linear contrasts are shown represented by volcano plots (Figure 4.4A) and delineated in Table 4.1. Specifically, BMDMs treated with sodium arsenite only resulted in one upregulated miR and two downregulated, while LPS treated BMDMs had eight upregulated and nine downregulated, respectively, compared to control (Figure 4.4A middle panel). Interestingly, when BMDMs were exposed to LPS+As(III) there were five upregulated and three down-regulated miRs compared to treatment with LPS alone (Figure 4.4A lower panel). A 2D scatter plot reveals a strong correlation of miRNA signals between LPS vs. LPS+As(III) treated BMDMs (Figure 4.4A lower panel inset; $r=0.627853)$. LEfSe analysis produced similar results for significant differentially expressed miRNAs (Figure 4.4B); data mining suggested potential biomarkers that explain differences between the treatment groups, filtered using the same threshold criteria of log2FC of 2 and $p$-value $>0.05$. Hierarchical clustering analysis of array data to identify patterns of expression between treatments is shown in heatmap (Figure 4.4C), with the LPS treatments segregating separately from the control and As(III) groups. For each treatment there was a set of unique 
miRNAs found to be differentially expressed only in response to that treatment suggesting treatment-specific differences in miRNA influences on pathological processes. 


\section{Figure 4.4. MicroRNA (miRNA) transcriptome analysis.}

Volcano scatter-plot, LEfSe plot, and heatmap visualization of microarray analysis of BMDMs treatment groups and linear contrast analysis. (A): Volcano plots illustrating log2FC compared with $p$-value (-log base 10) between As(III) vs. Control (upper panel), LPS vs. Control (middle panel), and LPS+As(III) vs. LPS (lower panel). Horizontal bar at $y=1.301$ represents threshold significance level of $P=0.05$; vertical cutoff of log2FC $>0.301$ (extreme right) and <-0.301 (extreme left). Lower panel inset shows scatter plot showing the relative spread of fold changes between LPS vs. LPS+As(III) treatments $(r=0.627853)$. (B): LEfSe analysis of same contrasts (with same threshold cutoffs) as volcano plot. (C): Heatmap showing miRNAs with statistically significant expression changes in all treatments. Hierarchical clustering analysis was used to identify patterns of miRNA expression in the As(III), LPS, and LPS+As(III) treatments. Heatmap scale bar indicates fold change range. 
A.
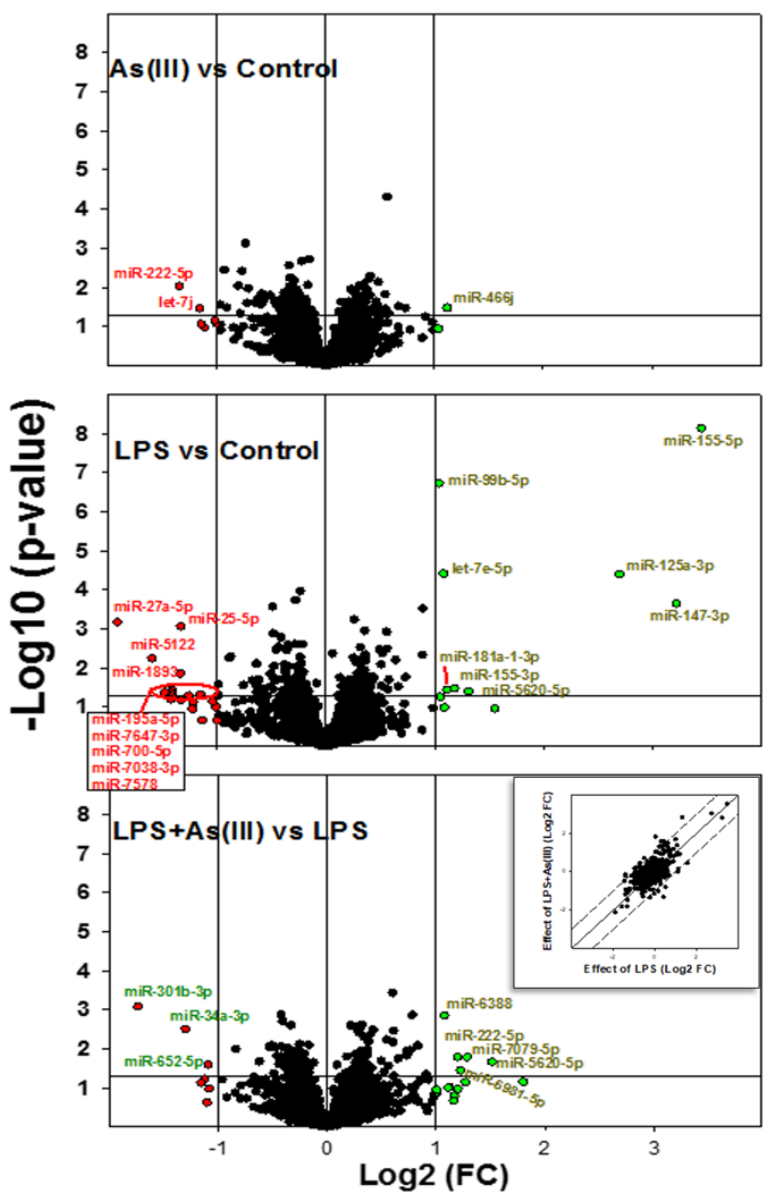

B.

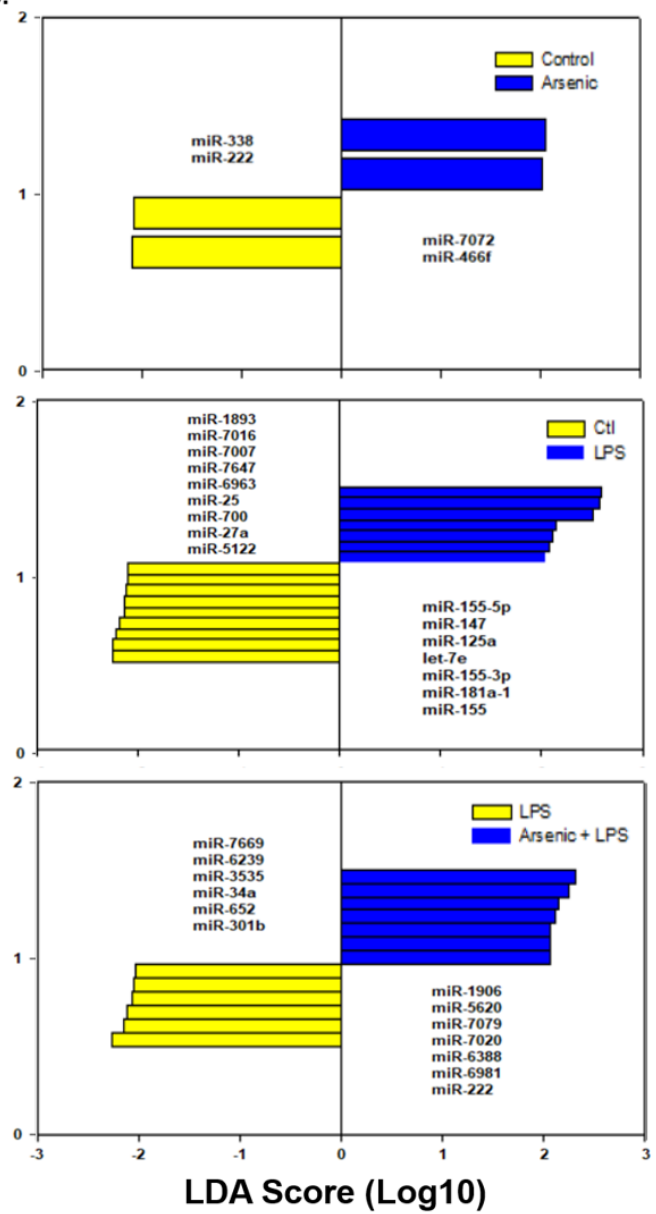

c.

Hierarchical Clustering - Euclidean Dissimilanty
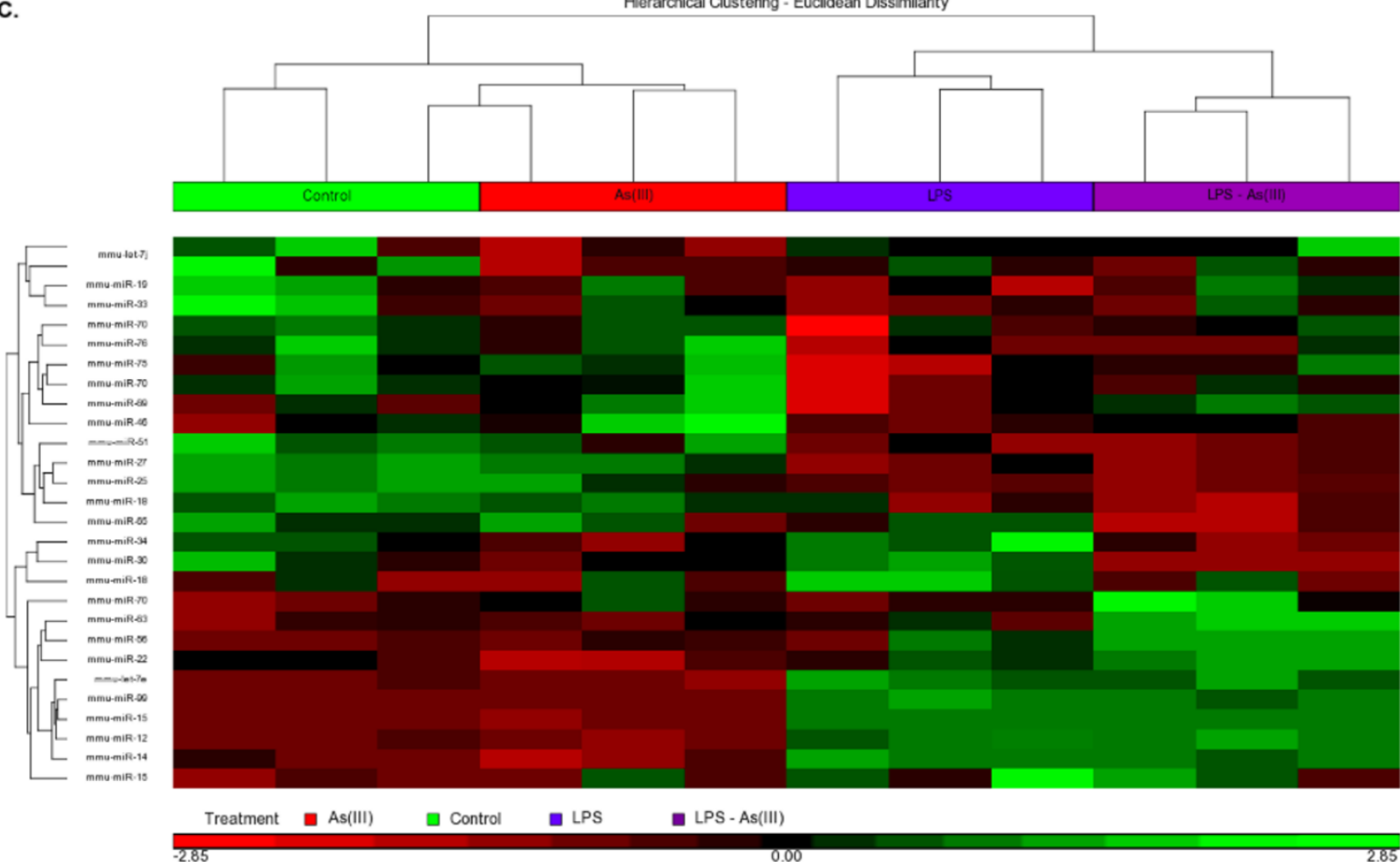
Table 4.1. Listing of differentially expressed miRNAs

\begin{tabular}{|c|c|c|c|}
\hline Contrast & miRNA ID & $p$-value & $\log 2 F C$ \\
\hline \multirow[t]{3}{*}{ As vs. C } & miR-466j & 0.033 & 2.172 \\
\hline & let-7j & 0.034 & -2.230 \\
\hline & miR-222.5p & 0.009 & -2.542 \\
\hline \multirow[t]{17}{*}{ LPS vs. C } & miR-155-5p & $7.370 \times 10^{-9}$ & 10.889 \\
\hline & mmu-miR-147-3p & $2.233 \times 10^{-4}$ & 9.293 \\
\hline & mmu-miR-125a-3p & $3.960 \times 10^{-5}$ & 6.480 \\
\hline & mmu-miR-5620-5p & 0.039 & 2.477 \\
\hline & mmu-miR-155-3p & 0.033 & 2.263 \\
\hline & mmu-miR-181a-1-3p & 0.036 & 2.157 \\
\hline & mmu-let-7e-5p & $3.810 \times 10^{-5}$ & 2.113 \\
\hline & mmu-miR-7038-3p & 0.047 & -2.222 \\
\hline & mmu-miR-25-5p & $8.557 \times 10^{-4}$ & -2.517 \\
\hline & mmu-miR-1893 & 0.014 & -2.525 \\
\hline & mmu-miR-339-3p & 0.036 & -2.664 \\
\hline & mmu-miR-7578 & 0.049 & -2.667 \\
\hline & mmu-miR-7647-3p & 0.042 & -2.684 \\
\hline & mmu-miR-700-5p & 0.042 & -2.759 \\
\hline & mmu-miR-195a-5p & 0.042 & -2.786 \\
\hline & mmu-miR-5122 & 0.006 & -3.024 \\
\hline & mmu-miR-27a-5p & $6.633 \times 10^{-4}$ & -3.765 \\
\hline \multirow[t]{8}{*}{ LPS+As vs. LPS } & mmu-miR-5620-5p & 0.022 & 2.856 \\
\hline & mmu-miR-7079-5p & 0.016 & 2.436 \\
\hline & mmu-miR-6981-5p & 0.036 & 2.337 \\
\hline & mmu-miR-222-5p & 0.016 & 2.295 \\
\hline & mmu-miR-6388 & 0.001 & 2.109 \\
\hline & mmu-miR-652-5p & 0.025 & -2.131 \\
\hline & $m m u-m i R-34 a-3 p$ & 0.003 & -2.463 \\
\hline & mmu-miR-301b-3p & $8.239 \times 10^{-4}$ & -3.330 \\
\hline
\end{tabular}




\begin{tabular}{|c|c|c|c|}
\hline Contrast & miRNA ID & $p$-value & $\log 2 \mathrm{FC}$ \\
\hline \multirow[t]{26}{*}{ LPS+As vs. As } & mmu-miR-147-3p & $6.920 \times 10^{-5}$ & 13.986 \\
\hline & mmu-miR-155-5p & $4.950 \times 10^{-9}$ & 12.327 \\
\hline & mmu-miR-125a-3p & $7.740 \times 10^{-6}$ & 10.307 \\
\hline & mmu-miR-222-5p & $7.302 \times 10^{-5}$ & 7.667 \\
\hline & mmu-miR-5620-5p & 0.002 & 5.657 \\
\hline & mmu-miR-1906 & 0.033 & 4.608 \\
\hline & mmu-miR-5620-3p & 0.014 & 3.231 \\
\hline & mmu-miR-29b-1-5p & 0.032 & 3.126 \\
\hline & mmu-miR-7070-5p & 0.006 & 2.764 \\
\hline & mmu-miR-7020-5p & 0.040 & 2.760 \\
\hline & mmu-miR-6971-5p & 0.003 & 2.451 \\
\hline & mmu-mir-155 & 0.026 & 2.411 \\
\hline & mmu-miR-6388 & $6.784 \times 10^{-4}$ & 2.315 \\
\hline & mmu-miR-149-5p & 0.025 & 2.263 \\
\hline & mmu-let-7j & 0.040 & 2.166 \\
\hline & mmu-miR-6954-5p & 0.001 & 2.165 \\
\hline & mmu-miR-6988-5p & $1.808 \times 10^{-4}$ & 2.096 \\
\hline & mmu-let-7e-5p & $5.710 \times 10^{-5}$ & 2.028 \\
\hline & mmu-miR-25-5p & 0.004 & -2.012 \\
\hline & mmu-miR-652-5p & 0.022 & -2.174 \\
\hline & mmu-miR-762 & 0.029 & -2.227 \\
\hline & mmu-miR-5122 & 0.014 & -2.520 \\
\hline & mmu-miR-3104-5p & 0.003 & -2.715 \\
\hline & mmu-miR-1893 & 0.008 & -2.784 \\
\hline & mmu-miR-27a-5p & 0.001 & -3.230 \\
\hline & mmu-miR-7648-3p & 0.010 & -3.233 \\
\hline
\end{tabular}

Table 4.1. Linear contrast results representing highest and lowest $\log 2 \mathrm{FC}$ values; threshold was miRNAs with significance of $p<0.05$, log2FC $=2$. Data generated from Affymetrix miRNA 4.0 GeneChip, and analyzed in R using the 
pd.mirna.4.0 package from Bioconductor and linear contrast with the limma function.

4. Differential miRNA expression profiles of $m i R-301 b$ validation experiments miR-301b was an immediate attractive target based on its expression profile in the LPS+As(III) vs. LPS linear contrast; it was downregulated with a $\log 2 \mathrm{FC}$ of -3.330 . Putative targets of miR-301b were mined from TargetScan and resulting gene lists were uploaded in DAVID for exploration of pathway associations. Top results are found in Table 4.2. Based on these results, genes in the TGF- $\beta$ signaling family were explored for their high degree of fold enrichment in predicted associated functional pathways, as well as their obvious relation to M2-like, anti-inflammatory phenotypes that may play a role in mitigating an inflammatory response. First, expression of miR-301b was evaluated in BMDMs from all treatment groups (Figure 4.5A). As expected, expression was significantly decreased in the LPS+As(III)-treated cells. Gene expression of putative gene targets were assessed as well (Figure 4.5B). Since miR-301b was downregulated in this contrast, target genes consequently were expected to be upregulated. INHB-A, the gene for a subunit of Activin A, has been shown to be associated with the mTOR signaling and transduction of TGF$\beta$ signaling $[173,174]$, along with RICTOR, a gene associated with the mTORC2 complex, were indeed both upregulated with treatment of LPS. With cotreatment, expression was significantly higher than both control and LPS treatment groups. The gene encoding the Activin type II receptor (ACVR2) was significantly upregulated only in the cotreatment group. Similarly, the BMP type II receptor 
(BMP2R), a key part of the TGF- $\beta$ signaling pathway, was also only upregulated in the cotreatment group, respective to all other treatments.

Table 4.2. DAVID pathway analysis.

\begin{tabular}{|c|c|c|c|}
\hline Gene Targets & Functional Pathways & $p$-value & $\begin{array}{l}\text { Fold } \\
\text { Enrichment }\end{array}$ \\
\hline $\begin{array}{l}\text { HOXB3, HOXB1, } \\
\text { HOXA3, HOXB2, } \\
\text { HOXA5 }\end{array}$ & $\begin{array}{l}\text { Homeobox protein, } \\
\text { antennapedia type, } \\
\text { conserved site }\end{array}$ & 0.009 & 5.950 \\
\hline $\begin{array}{l}\text { TGFBR1, TGFBR2, } \\
\text { PPARG }\end{array}$ & Response to lipid & 0.004 & 27.451 \\
\hline $\begin{array}{l}\text { ACVR2A, TGFBR2, } \\
\text { ACVR1, ACVR1C }\end{array}$ & Activin type II receptor & 0.005 & 21.418 \\
\hline $\begin{array}{l}\text { MAPK1, TSC1, } \\
\text { PIK3CB, ULK2, } \\
\text { PRKAA1, PRKAA2 }\end{array}$ & mTOR signaling pathway & 0.035 & 3.253 \\
\hline $\begin{array}{l}\text { E2F2, TNF, ESR1, } \\
\text { NEUROG1, PDE3A, } \\
\text { PTEN, TGFB2, } \\
\text { ADCYAP1, EREG, } \\
\text { CCND3, DCUN1D3, } \\
\text { SIK1, GADD45A, } \\
\text { RUNX3 }\end{array}$ & Regulation of cell cycle & 0.049 & 1.796 \\
\hline $\begin{array}{l}\text { TNF, TGFBR1, } \\
\text { TGFBR2, SMAD5, } \\
\text { BMPR2, SMAD2, } \\
\text { TGFB2, ACVR1C, } \\
\text { INHBB, INHBA, } \\
\text { MAPK1, ACVR2A, } \\
\text { SP1, ZFYVE9, } \\
\text { SMURF2, ACVR1 }\end{array}$ & $\begin{array}{l}\text { TGF-beta signaling } \\
\text { pathway }\end{array}$ & $1.661 \times 10^{-7}$ & 5.384 \\
\hline $\begin{array}{l}\text { TNFRSF1B, ACSL1, } \\
\text { TNF, RXRA, PRKAB1, } \\
\text { PRKAA1, PRKAA2, } \\
\text { ACSL4 }\end{array}$ & $\begin{array}{l}\text { Adipocytokine signaling } \\
\text { pathway }\end{array}$ & 0.007 & 3.496 \\
\hline $\begin{array}{l}\text { TNF, IL6ST, TGFBR1, } \\
\text { CSF1, TGFBR2, IL25, } \\
\text { BMPR2, KIT, TGFB2, } \\
\text { INHBB, INHBA, }\end{array}$ & $\begin{array}{l}\text { Cytokine-cytokine receptor } \\
\text { interaction }\end{array}$ & 0.018 & 1.920 \\
\hline
\end{tabular}




\begin{tabular}{|l|l|l|l|}
\hline $\begin{array}{l}\text { ZFP91, ACVR2A, } \\
\text { TNFRSF1B, PDGFRA, } \\
\text { ACVR1 }\end{array}$ & & & \\
\hline $\begin{array}{l}\text { ACVR2A, TGFBR1, } \\
\text { TGFBR2, BMPR2, } \\
\text { ACVR1C, ACVR1 }\end{array}$ & TGF-beta receptor type I/II & $2.380 \times 10^{-5}$ & 15.303 \\
\hline $\begin{array}{l}\text { ACVR2A, TGFBR1, } \\
\text { BMPR2, ACVR1C, } \\
\text { ACVR1 }\end{array}$ & $\begin{array}{l}\text { TGF-beta receptor/activin } \\
\text { receptor, type I/II }\end{array}$ & $4.010 \times 10^{-4}$ & 12.981 \\
\hline
\end{tabular}

Table 4.2. Top pathways enriched by DAVID pathway analysis with predicted functional pathways associated with corresponding expression array data.

Putative gene target predictions extracted from TargetScan. Gene annotation, ontology, and pathway analysis via DAVID. A modified Fisher's exact test/EASE (Enrichment) Score was utilized to calculate the $p$-values. 
Figure 4.5. Validation of miRNA expression profiles of treatment groups. RNA was harvested from BMDMs treated for $6 \mathrm{~h}$ with either As(III), LPS, or cotreatment with both, was evaluated for mRNA expression via qRT-PCR. (A): mRNA expression of miR-301b in BMDMs from control and treatment groups; ${ }^{a}$, $p<0.05$, compared to control mRNA expressions; $b, p<0.05$, compared to respective LPS treatment. (B): qRT-PCR for putative gene targets of miR-301b; as expected, downregulation of miR-301b correlated to upregulated mRNA expression of target genes. INHBA (Activin A), ACVR2 (Activin Receptor) RICTOR (mTOR complex), and BMPR2 (BMP Receptor), all increased significantly with respect to control and LPS treatment. Quantitative data are shown as means \pm SEM $(n=3)$. These genes are associated with the TGF- $\beta$ and BMP pathways, which downregulate IL-12 in murine MФ and suppress LPSinduced TLRs. 
A.

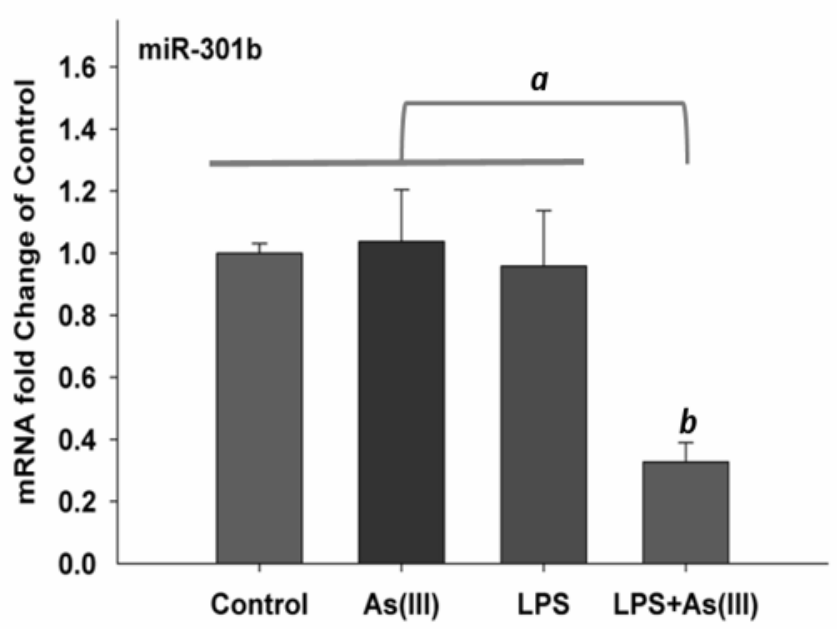

B.

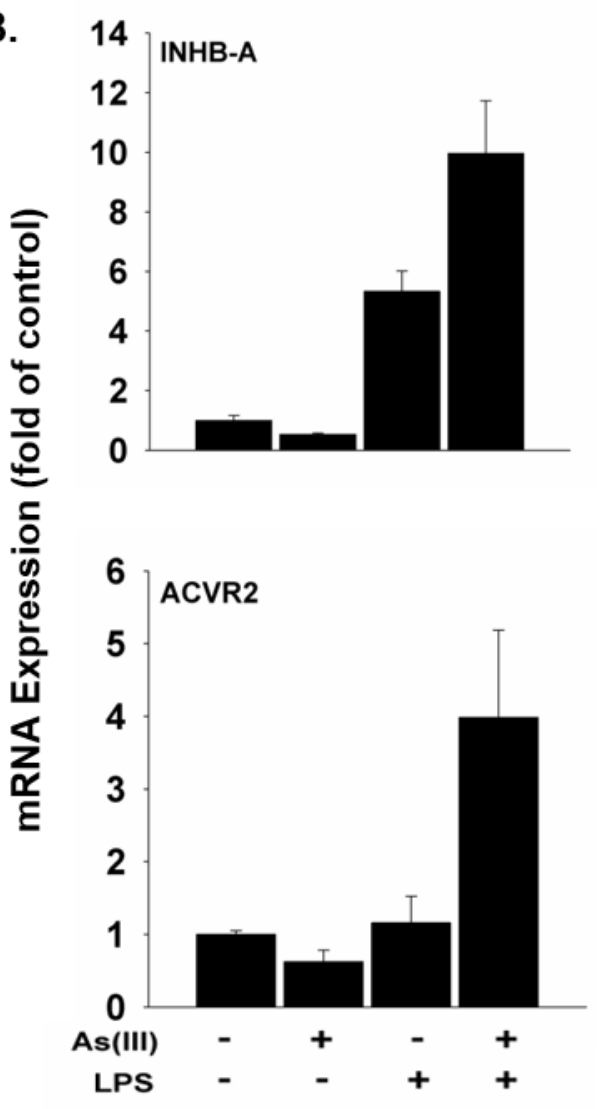

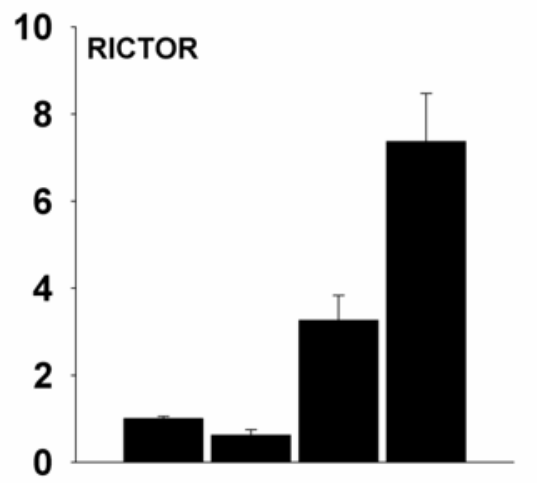

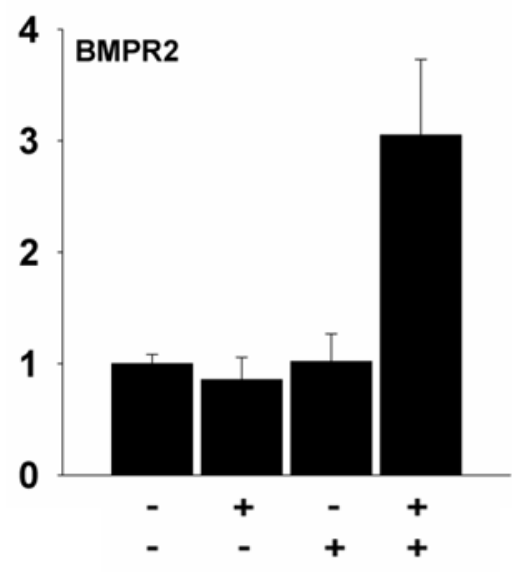




\section{Gene ontology}

Networks identified via IPA core and comparative analyses have some interesting relations to innate immunity and anti-inflammatory pathways. miR$301 b-3 p$ is represented as miR-130 which is the family in which $301 b$ is classified, with other miRNAs of shared seed regions. Compartmentally, this miR was identified to act in the cytoplasm. In the contrast LPS+As(III) vs. LPS, two networks were generated that included the miR-130 family. Network A (Figure 4.6A) was comprised of molecules associated with connective tissue disorders, along with the inflammatory response and disease, while Network B (Figure 4.6B) classified interrelated miRNAs and their targets into top diseases and functions implicated in inflammatory disease, inflammatory response, and organismal injury and abnormalities. Included in Network A is TP53, a tumor suppressor protein that regulates cell proliferation, which acts on miR-130. miR222-5p, which was upregulated in this contrast (Table 4.1), also has some indirect interactions with miR-130 via AGO2 and PGPEP1L (Figure 4.6A).

The key molecules in Network B include insulin, which acts indirectly to affect miR-130 expression, and also Smad2/3 from BMP/TGF- $\beta$ pathways. Importantly, miR-130 it has a direct interaction with the Activin type II receptor, which is also self-regulating. miR-130 in turn acts on TMEM250 which positively regulates proliferation via potential role in promoting $S$ phase in cell cycle, along with MDM4, an inhibitor of the p53 tumor suppressor protein. MTMR9 is directly acted on by 130 as well, and it too plays a role in cell proliferation.

Also identified in Network B was miR-34a-3p (Table 4.1), a miR that was also downregulated in the LPS+As(III) vs. LPS contrast; notably, this miR was 
also classified in an overlapping network where it interacts with the TGFB1 ligand as well as IL-10, both of which are associated with M2 polarization. Upon association of the TGFB1 ligand with the TGF- $\beta$ receptor, SMAD family molecules are activated, initiating the key components of the TGF- $\beta$ pathway. Toxicity functions identified indicated links to cardiac, hepatic, and renal inflammation, hyperplasia/cancer, liver steatosis, and fibrosis/cirrhosis (Figure $4.6 \mathrm{C})$. 


\section{Figure 4.6. IPA network analysis.}

Network analysis for LPS-As vs. LPS contrast was completed in IPA suite.

Network panels generated from a Disease Biofunctional analysis with filters selected for inflammation and innate immunity. (A): Network A includes the TGF$\beta 1$ ligand gene, associated with the TGF- $\beta$ pathway and SMAD family expression. Here miR-301b is represented as miR-130, its classification family which includes all other miRNAs with the same shared seed regions. Included in this network is TP52, a tumor suppressor protein that regulates cell proliferation, and miR-222-5p, which was upregulated in the LPS+As(III) vs. LPS contrast. (B): Network B includes insulin as an effector of miR-130 expression, as well as Smad2/3 from the BMP/TGF- $\beta$ pathways. Here miR-130 also acts on TMEM250 and MTMR9, which both positively regulate cell proliferation, as well as MDM4, an inhibitor of the p53 tumor suppressor protein (C): Toxicity function analysis identifying key clinical pathological endpoints and toxicity mechanisms. 
A.

miR-344i (miRnAs wiseed AGUCAGG)*

miR-344h-5p (and other miBNAs w/seed AGGCUUC)*

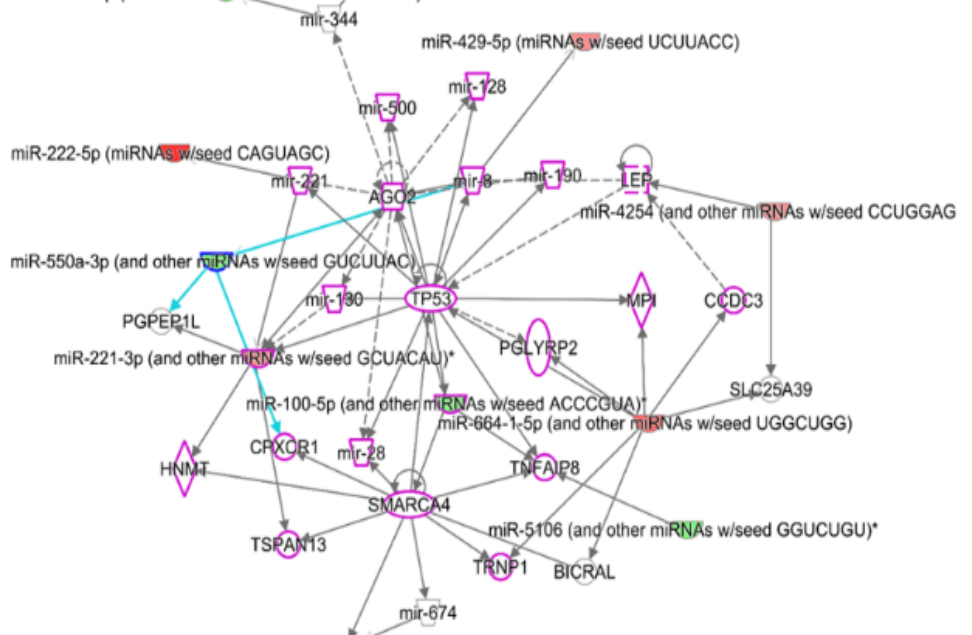

miR-674-5p (and other miRNAS w/seed CACUGAG)*

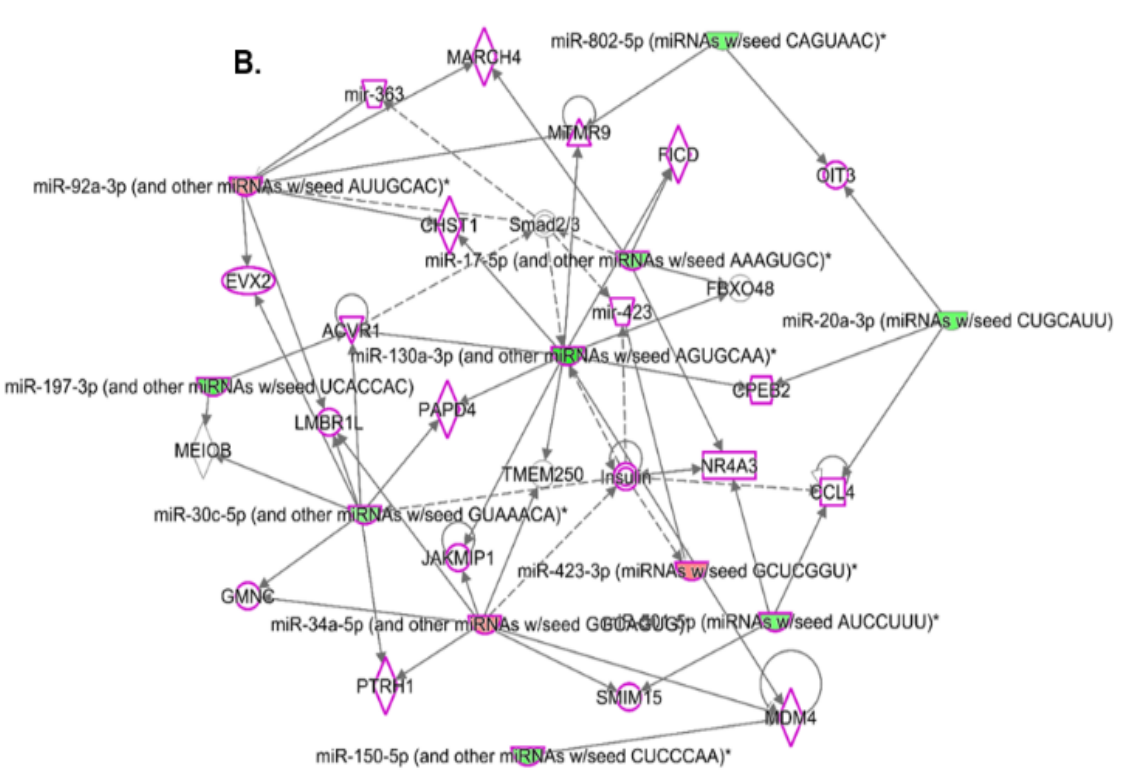

C. ILPS Assenic vs. LPS

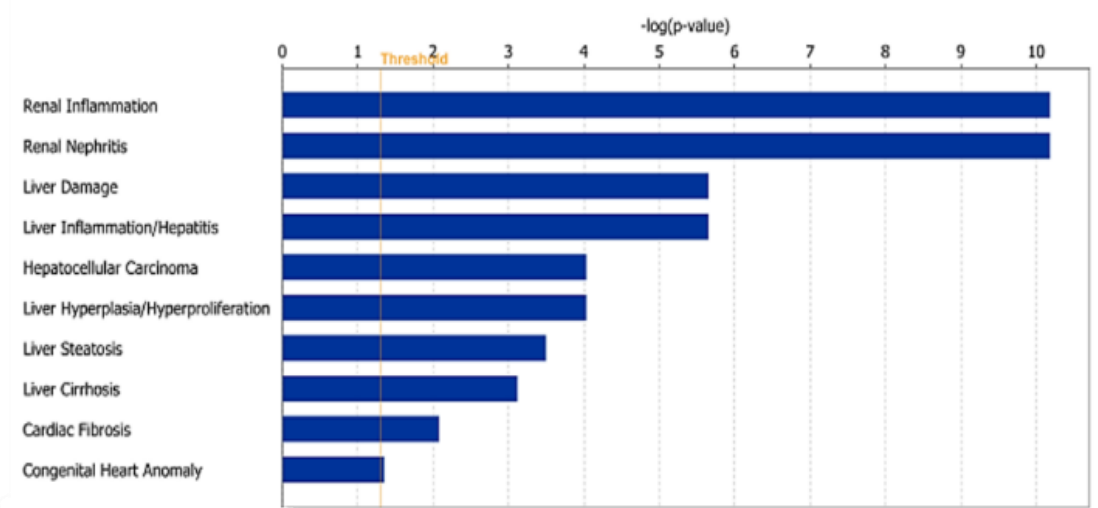


Figure 4.7. IPA Diseases and Biofunctional analysis.

Comparative Ingenuity Pathway Analysis of top diseases and biofunctions associated with miRNA expression dataset. (A): Comprehensive comparative analysis of disease and biofunctions identified in all contrasts. (B): Comparative analysis of disease and biofunctions specific to LPS+As(III) vs. LPS contrast. 


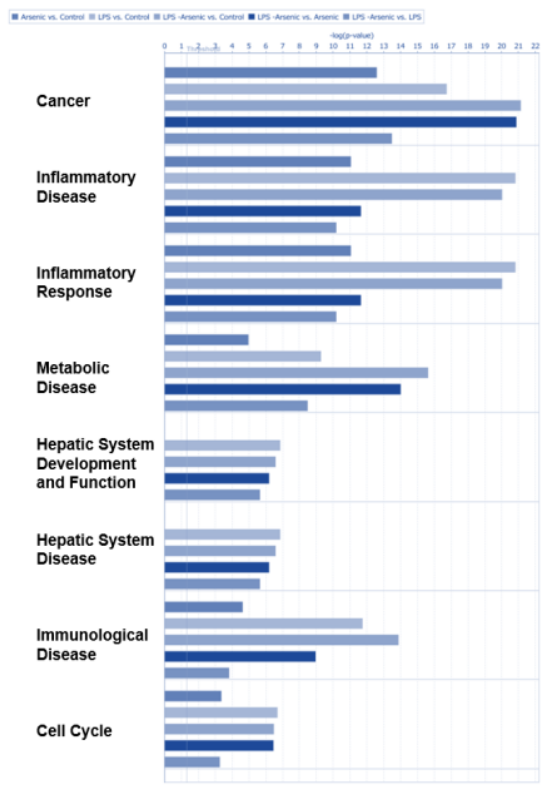

B. ILS -Arenicicis LPS

Organismal Injury \& Abnormalities

Reproductive System Disease Cancer

Inflammatory Disease

Inflammatory Response

Renal \& Urological Disease

Endocrine System Disorders

Gastrointestinal Disease

Metabolic Disease

Connective Tissue Disorders

Respiratory Disease

Developmental Disorder

Hereditary Disorder

Digestive System Development \& Function

Hepatic System Development \& Function

Hepatic System Disease

Infectious Diseases

Organ Development

Neurological Disease

Psychological Disorders

Hematological Disease

Cellular Development

Cellular Growth \& Proliferation

Immunological Disease

Cell Cycle

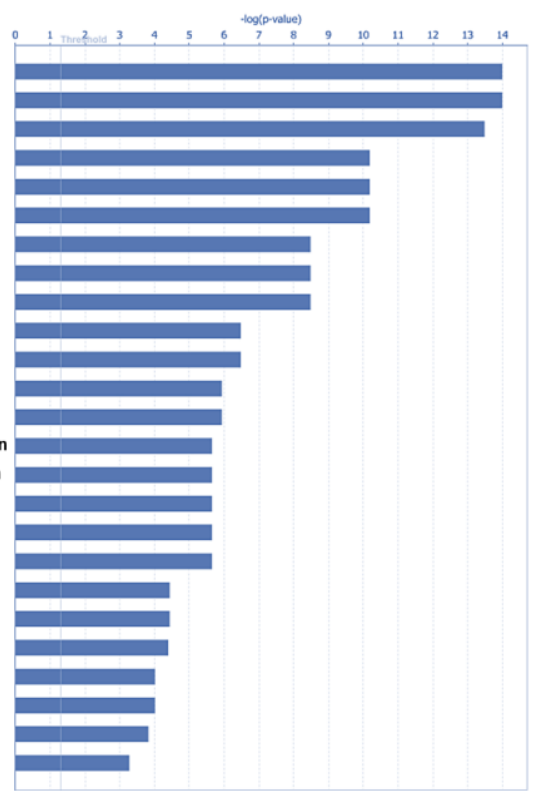




\section{Discussion}

As mentioned in the introduction, chronic exposure to arsenic in drinking water results in carcinogenicity, neurological dysfunction, cardiovascular disorders, reproductive defects and potentially diabetogenic effects. All of these disorders are impacted, at least in part, via altered function of the innate immune response. It has been previously demonstrated that arsenic is a potent immunomodulatory agent in many experimental models and epidemiologic studies [175-179], for example, in altering the innate immune system in response to low dose arsenic exposure in mice [180]. Macrophages are highly specialized cells that perform numerous tasks in the innate immune system for example phagocytosis, antigen presentation, cytokine production, and migration [181183]. The potential impact of arsenic exposure on macrophage function is currently poorly understood. The results herein indicate that low concentration of arsenite attenuated the LPS-mediated induction of CD11c expression, a marker of M1 polarization, in addition to the associated abrogation of classic proinflammatory markers in treated macrophage populations. In agreement with these findings, low dose arsenic exposure was reported to attenuate the innate immune response to influenza $A(\mathrm{H} 1 \mathrm{~N} 1)$ viral infection in mice with a significant decrease in the total number of $C D 11 \mathrm{C}+/ C D 103+$, and $C D 11 \mathrm{C}+/ \mathrm{B} 220+$ dendritic cells [180].

The BMDMs are an excellent model to study several mechanisms in primary cell culture. Compared to many other primary cells, the BMDMs are homogenous cells, possess proliferative capacity, can be transfected, and have a 
relatively long lifespan without noticeable changes in cellular viability or morphology [184]. Other studies in published literature have employed Kupffer cells or RAW 264.7 cells to study polarization reversal or reprogramming [185]. Although RAW 264.7 cells are viewed as unstimulated macrophages, they are reported to have low basal $[186,187]$. These are also immortalized cells that may differ in response than primary cells. Isolated macrophages (e.g., Kupffer cells or peritoneal macrophages) are at least partially polarized by their vivo environment, as well as potentially being influence by the isolation procedure $[187,188]$. BMDMs provide a platform to evaluate uncommitted primary naïve macrophages in culture.

In attempt to examine the impact of arsenite exposure on the stimulated polarization of bone-marrow derived macrophages, cells treated with LPS or LPS+As(III) were evaluated for M1 activation, and cells treated with IL-4 or IL$4+\mathrm{As}(\mathrm{III})$ were used to model the alternative M2a response. Flow cytometric analysis showed that arsenite alone did not significantly affect the number of $\mathrm{CD} 11 \mathrm{c}+/ \mathrm{F} 480+/ \mathrm{CD} 11 \mathrm{~b}+$ cells or the MFI of CD11c as a marker of M1 polarization. However, arsenite significantly attenuated the increase in the number of $\mathrm{CD} 11 \mathrm{c}+/ \mathrm{F} 480+/ \mathrm{CD} 11 \mathrm{~b}+$ cells at all concentrations tested, with a maximal effect at $100 \mathrm{ppb}$. This effect of arsenite is independent of cytotoxicity, as arsenite treatment at all concentrations, with the exception of $500 \mathrm{ppb}$, did not cause any significant reduction in BMDMs cellular viability. In contrast, arsenite did not affect the number of CD206+/F480+/CD11b+ BMDMs or the intensity of CD206 in the presence/absence of IL-4. These results suggest that arsenite 
alone does not affect macrophage polarization, but has the potential to modify or deregulate the toll-line receptor 4 (TLR4)-mediated M1 response.

The term 'macrophage polarization' implies a potentially binomial response of macrophages to stimulation/activation; "M1" or "classically-activated" macrophages are viewed the predominant proinflammatory phenotype, and the "M2" or "alternatively activated" is viewed as the predominant anti-inflammatory phenotype. However, it is understood that this paradigm is likely an oversimplification. Specifically, it is understood that there is a continuum of phenotypes between "pure" M1 and M2 stages [189]. Furthermore, transcriptome analysis has revealed that there may be many more phenotypes than simply M1 or M2 [190]. Thus, it is quite possible to impact the differentiation of macrophages to one phenotype without affecting the response to other stimuli. Indeed, here arsenic exposure appeared to impair the response to LPS (i.e., "M1") without noticeably impacting indices in response to IL-4 (i.e., "M2").

Arsenic has been previously demonstrated to decrease cell adhesion and migration genes, pro-inflammatory genes such as IL-1 [180], and nitric oxide (NO) production through iNOS [191, 192]. In this study, arsenic-treated cells exhibited an attenuation of the LPS-mediated induction of the M1 prototypical pro-inflammatory genes TNF- $\alpha$, IL- $\beta$, iNOS in addition to PAI-1 (Figure 4.3). Interestingly, arsenite alone increased PAI-1 expression, while it attenuated the LPS-mediated induction of PAI-1, suggesting the presence of separate mechanisms of PAI-1 regulation in BMDMs. Classically-activated M1 macrophages are often associated with acute infection, contributing to the 
development of microbicidal activity [193]. It is thus plausible that impairment of M1 polarization by arsenite might explain the increased susceptibility to infection and mortality due to bacterial infection upon arsenic exposure [194].

miRNAs are mediators of gene regulation that are key biomarkers in tissue-specific gene expression and pathogenesis of disease [195, 196]. miRNAs are small, endogenous, non-coding RNA molecules of $\sim 18-25$ nucleotides that post-transcriptionally regulate gene expression [169]. It is currently known that miRNAs are involved in the regulation of maturation, proliferation, differentiation and activation of both the innate and adaptive immune cells [186]. miRNAs contribute to activation and polarization of macrophages and permit for an initially strong immune response which is gradually diminished, thus providing a possible advantage over other TLR regulatory mechanisms [188]. The inflammatory response is well characterized for microbial infections, especially in the case of LPS, which binds to the innate immune receptors TLR4 [197]. Sufficient production of inflammatory mediators by M1 macrophages is essential to clear pathogenic infection; therefore, restraining TLR responses can have major deleterious effects. The modulation of the balance between M1:M2 activation states has been the focus of numerous studies, particularly with regard to transcriptional and epigenetic regulation through microRNAs $[171,186]$.

Microarray analysis of LPS treatment produced data similar to what has been previously described for miRNAs associated with TLR4 activation and inflammatory murine macrophages [198]. The current study is the first to report the effect of arsenite exposure on miRNA expression in BMDMs. In particular, 
miR-155, miR-21 and miR-146a have been identified as primary players due to their expression levels following TLR activation [170, 199-201]. Indeed, in our analysis, miR-155-5p was the most upregulated miR in cells treated with only LPS (with respect to control cells), with a log2FC of 10.90 and a $p$ value of $7.37 \times 10-9 ;$ miR-155-3p was also significantly upregulated at a $\log 2 \mathrm{FC}$ of 2.26 and $p$ value 0.03 . Another key miRNA, miR-147 was upregulated in the linear contrasts of LPS vs. C and LPS+As(III) vs. As(III) alone; this miR is induced with TLR activation and has been reported to regulate inflammation in murine macrophages [202, 203]. Other miRNAs have been also identified to be up regulated in response to TLR ligands such as miR-132 [204], miR-9 [205], miR147 [202] and miR-346 [206]. miRNA analysis of the contrasts LPS vs. C and LPS+As(III) vs. As(III) alone indicated an upregulation of miR-125a-3p, which is consistent with its role in differential activation of inflammatory murine macrophages [207]. miR-181, another effector of the TLR4 pathway, was also upregulated with the LPS vs. $C$ contrast $(\log 2 F C=2.16 ; p=0.04)$. let-7e-5p was also upregulated in LPS-treated cells, consistent with its regulation by Akt1, which is activated by LPS [201].

The linear contrast comparing miRNA expression of LPS+As(III) cells vs. those treated with LPS alone revealed that miR-301b-3p as the most downregulated (Table 3.1), with a log2FC of -3.33 and $p$ value of 0.0008 . This miR was chosen for follow up analysis and exploration. First, it was verified as being downregulated by comparing all treatment groups via qRT-PCR quantitation. Putative gene targets identified in this group include ACVR2A, 
TGFBR1, BMPR2, ACVR1C, ACVR1, INHBB, and INHBA. We measured ACVR2, INHBA, INHBB, BMPR2, ACVR2, RPTOR and RICTOR to determine expression in treated and untreated cells (Figure 4.5). Gene ontology analysis revealed a significant enrichment for the mTOR signaling pathway, TGF- $\beta$ receptors, cytokine signaling, and cell cycle activation; the activin A receptor type I/II was selected group of gene targets for evaluation. Activin A has been shown to potentially function in regulating macrophage polarization switching [208]. Activin A suppresses LPS-induced TLRs, cytokines and iNOS by inhibition of NF$\kappa \beta$ and MAPK [209]. It has been reported to skew macrophage polarization by promoting a proinflammatory phenotype and inhibiting the acquisition of antiinflammatory macrophage markers [210, 211].

IPA analysis indicated strong correlations with cancer for the miR-130a family, which may be related to the increased expression of TH2-like cytokines of tumor cells coopting tumor associated macrophages (TAMs), and the exploitation of their tissue trophic and repair functions by invading tumor cells [212] (Pollard, 2018). The disease and biofunction IPA analysis produced top results for various cancers, including squamous cell carcinoma, cervical cancer, thyroid carcinoma, leukemia, and colorectal cancer. Metastatic melanoma, as well as general autophagy, and metastasis, were identified; tumor migration and metastatic activation z-scores were the highest among the biofunctions classified in the LPS+As(III) vs. LPS contrast.

Arsenite by itself did not significantly affect BMDMs polarization, however cotreatment with LPS, arsenite was attenuated the LPS-induced M1 polarization. 
Taken together, activin A alone promotes classical activation of macrophages; whereas in macrophages that have already been activated to a proinflammatory state, it can attenuate the TLR-4 mediated M1 response. It is very possible that modulation of miRNAs, as shown in this study, controls the action of activin A in polarization switching; in this case, arsenic exposure upregulated expression of activin $\mathrm{A}$, and subsequently the M1 polarization of treated macrophages was altered. This effect of arsenite could potentially be attributed to its effects on miRNA expression profiles of macrophage populations. In the context of human exposure, humans are exposed to relatively similar levels of arsenite used for in vitro work here, and can even exceed this level if consumption of food containing arsenic in addition to the exposure to arsenic contaminated air and water are taken in to consideration. Based on these findings, it is proposed that low dose arsenic exposures could attenuate the proinflammatory response of the innate immune system, which in this system may indicate potential impairment of the bactericidal macrophage activity in vivo. Further investigation is needed to elucidate the specific pathway by which the arsenic-induced downregulation of miR-301b acts to attenuate that classic inflammatory macrophage response in vitro. 


\title{
CHAPTER V
}

\author{
EVALUATION OF IMMUNOMODULATORY INTERACTIONS WITH \\ MACROPHAGE POPULATIONS AND EFFECTS OF THE EXTRACELLULAR \\ MATRIX IN THE TUMOR MICROENVIRONMENT VIA A SPATIO-TEMPORAL \\ MATHEMATICAL MODEL OF TUMOR GROWTH
}

A. Introduction

The study of liver diseases and liver cancers and metastases often overlap, not only in underlying etiologies, but also in potential mechanisms of action. The causes of liver disease include alcohol or toxicant exposure, obesity, and viral infection, among a host of cofactors (Scheme 5.1); liver cancer, which is often preceded by liver diseases, also shares these underlying causes. Mechanistically, changes to the hepatic extracellular matrix (ECM) appear to play key roles, both oncogenic and nononcogenic [29]. These changes, in general, are mediated by a modulation of the normal balance between enzymes that degrade the ECM (e.g., matrix metalloproteinases; MMPs) and inhibitors of matrix degradation (e.g. tissue inhibitors of metalloproteinases; TIMPs). In liver fibrosis, TIMPs are upregulated and MMPs impaired, so that the balance tips towards aberrant matrix deposition. In cancer progression, the balance between these enzyme families is more nuanced; although cancers tend to induce MMP 
activity to facilitate tumor cell invasion, they often also establish novel ECM in the stroma of the neoplastic burden.

The cancer-specific ECM creates a niche for a tumor-specific microenvironment $[9,67]$. Both primary tumors and metastatic lesions are accompanied by dense desmoplasia resulted from chronic trophic macrophage signaling from circulating monocytes, and repolarization of nearby and recruited macrophage populations [20, 21]. These cells are all subverted by the tumor to facilitate neoangiogenesis. The excessive deposition of ECM in the tumor microenvironment is not solely attributable to activated fibroblastic cells, such as in liver disease, but also with nonfibrogenic cells that have undergone an epithelial-mesenchymal transition (EMT)[5]. In the economical fashion that is inherent to nature, many of the processes derived from a regression to/recovery of stem-like properties that promote unchecked growth, are then exploited by tumor cells to evade normal mechanisms of cell death signaling and immune function. Similarly, remodeling of liver ECM both in the context of normal repair and regeneration as well as under pathological conditions, uses developmental pathways highly associated with cancer progression [7]. This modified microenvironment may confer proliferative, invasive, and migratory potential to the tumor $[80,95]$.

Both macrophage signaling and ECM turnover in cancer and in liver diseases, involve the actions of macrophage modulation of the microenvironment [76], induced via their polarization to either classical M1, or proinflammatory activation state, or the so-called alternative M2 anti-inflammatory activation state. 
While it has been shown that macrophage polarization states range over a spectrum of phenotypes [188], with the M2 populations being capable of exhibiting a host of subtypes [193], in the context of cancer and TAMs, the perivascular TAMs present with an M1 type cell surface expression phenotype, whereas the stromal macrophages bordering hypoxic regions, show a more M2 trophic phenotype $[87,90]$. These M2-like TAMs are those responsible for mediating the signaling from hypoxic cells, and subsequently inducing expression of proangiogenic factors such as vascular endothelial growth factor (VEGF) and angiopoietin 2 (Ang2)[213, 214]. These signaling events can be modeled alongside the physical properties of the ECM, including its composition, stiffness, density, and structure. These features all become useful parameters in computational modeling that allow in silico exploration of various modulations and their effects on tumor proliferation. Using the ECM as a framework, or scaffold for cell growth, modeling of diffused molecules and signal switching can be added to simulation to explore tumor growth with respect to the effects of other components of the tumor microenvironment while modulating ECM turnover. The role of innate immune cells is one key interaction that is closely related to ECM remodeling and influencing tumor progression via neovascularization. Mathematical modeling of the ECM properties, and its interaction with macrophages, using the conceptual binary model of macrophage polarization, allows for simplification of describing the action of TAMs for the purposes of elucidating their interactions with the ECM. 
Here, we validate a mathematical model that assesses the heterogeneous macrophage population interactions with tumor cells, considering both control ECM and a profibrotic ECM, as could be associated with liver injury [215]. We perform experiments to calibrate the model to correlate simulations to in vitro and in vivo experiments. In the model, the ECM varies in thickness, modeled by simulating differing ratios of ECM degradation/production, the estimated parameters of which we base on a mouse model of transitional ECM remodeling resulting from chronic alcohol exposure. Macrophage behaviors simulated in the model include M1 release of TNF- $\alpha$ proinflammatory cytokine, M2 release of TGF- $\beta$ anti-inflammatory factors, both of which were calibrated based on data from macrophage-tumor cell indirect co-cultures. In addition, general tumor angiogenic factors that facilitate angiogenesis and promote tumor growth are part of the model environment. 


\section{Scheme 5.1. Natural history of liver disease.}

Regardless of etiology, the progression of pathology is comparable, with clinical/histological features shared across the spectrum. Progression from normal homeostasis to steatosis occurs in response to short term exposure and is reversible, characterized by presence of large sharp fat droplets in hepatocytes. In a small percentage of steatotic livers, persistent injury can lead to steatohepatitis, in which fatty livers develop parenchymal inflammation and hepatocellular damage. Continued inflammatory stimulus promotes aberrant fibrin deposition characteristic of fibrosis/cirrhosis, and formation of cirrhotic nodules then precede the development of hepatocellular carcinoma. Besides therapeutics for fibrotic regression or liver transplantation, there are limited treatment options for end stage disease. 


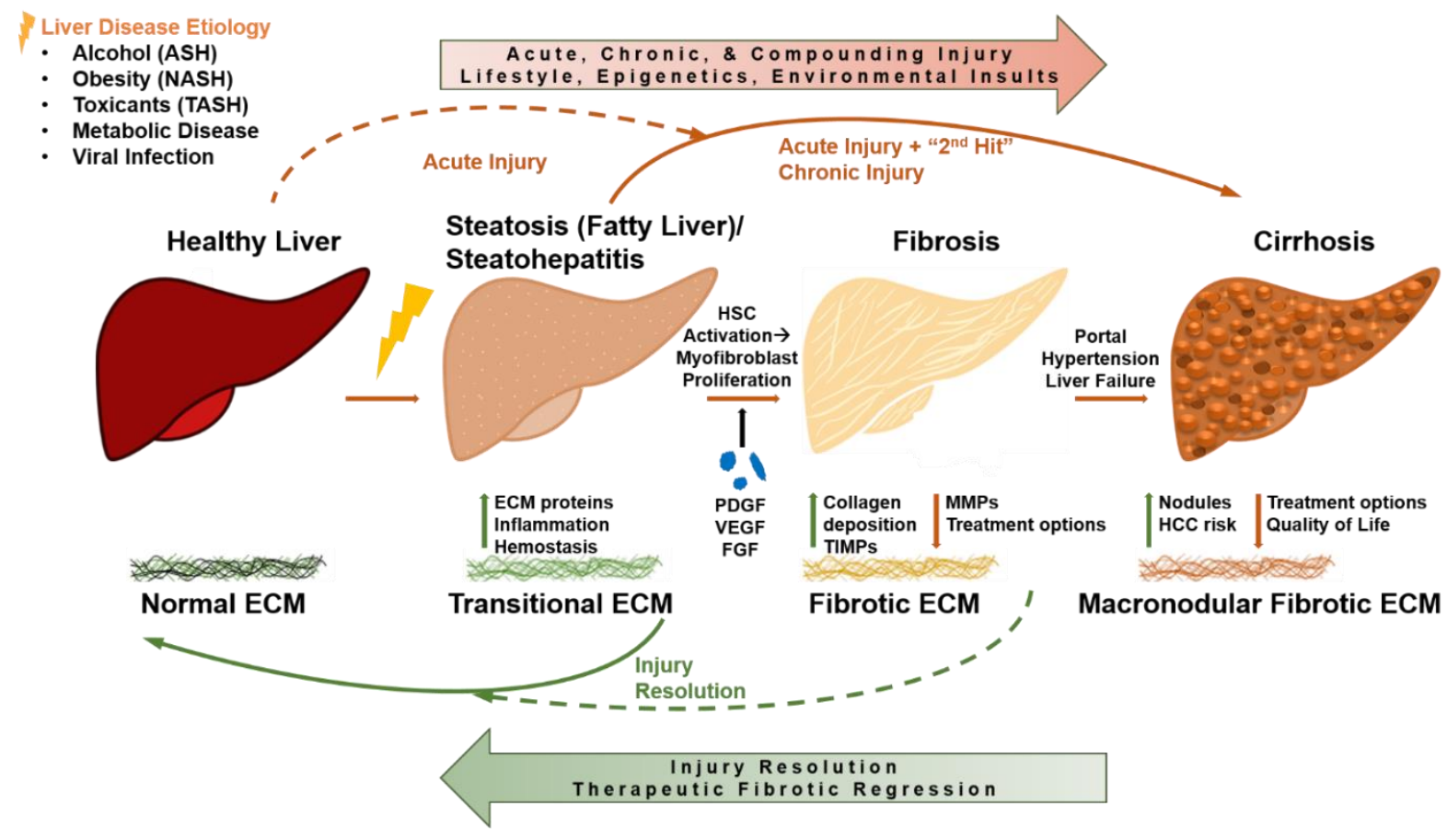




\section{B. Experimental Procedures}

1. Animals and treatments

Male C57BI6/J mice (6 wk) were obtained from the Jackson Laboratory (Bar Harbor, ME). Animals underwent Lieber-DeCarli alcohol diet maintenance as described in Chapter II, Section A.3. Liver tissues harvested and processed as described in Chapter II, Section H.1-2.

2. Histology

Liver tissues processed as indicated in Chapter II, Section B.1-3.

3. BMDM cell culture

Cells isolated and cultured as indicated in Chapter II, Section G.2.

4. Tumor cell culture and transwell assays

Cells cultured as indicated in Chapter II, Section G.4-5.

5. ELISA

ELISA assays completed as described in Chapter II, Section G.6.

6. Tumor simulations

Tumor growth simulations were conducted as described in Chapter II, Section D.2. The tumor growth component is based on a model developed by Macklin et al. [216]. The simulation matrix consists of a 2D grid of vasculature, 
evenly spaced $(250 \mu \mathrm{m})$, representing the normal capillary network [217]. The microenvironment is described as follows:

- Necrotic region-non-viable tumor oxygenation levels

- Hypoxic region-non-proliferating tumor oxygenation levels

- Normoxic region-sufficient oxygenation levels for tumor proliferation

- Normal-non-cancerous tissue

Tumor progression over time is modeled with proliferation dependent on changes to the microenvironment, including pressure, angiogenic factors, and oxygen concentration.

Tumor tissue advances in space with a certain velocity, $v_{c}$, through the surrounding normal tissue based on Darcy's law [216]:

$v_{c}=-\mu \nabla P+\chi_{E} \nabla E$,

where $\mu$ equates to tissue mobility (cell-cell, and cell-matrix linkages); $P$ is oncotic pressure, $\chi_{E}$ is haptotaxis constant, and $E$ is ECM density. If uniform density is assumed, the net tumor proliferation rate can be non-dimensionalized as $\lambda_{p}$, and the relationship between velocity and tumor growth is [216]:

$\nabla \cdot v_{c}=\lambda_{p}$

With nominal oxygen levels, proliferation distal from vasculature slows, hypoxic tissue regions, $\Omega_{\mathrm{H}}$, are created and from these are released the tumor angiogenic 
factors (TAFs), that diffuse through tumor tissue into surrounding matrix, where they instigate capillary sprouts from endothelial cells as well as vascular extravasation of macrophages [218], which correlates to VEGF-recruited macrophages diverted by tumor.

Simulations dictate monocytes extravasating from vascular grid in proportion to concentrations of macrophage chemoattractants produced by the hypoxic tumor tissue. The monocytes migrate towards tissue regions along increasing gradient of chemoattractant. Polarization to M1 or M2 phenotype occurs in the tumor microenvironment, relative to cytokine concentration released by $\Omega_{\mathrm{H}}$ and $\Omega_{\mathrm{P}}$ regions [215]. Monocytes and differentiated macrophages are treated as discrete agents, simulated via a cellular automaton algorithm.

Table 5.1 Macrophage-associated cytokines

\begin{tabular}{|l|l|l|l|l|}
\hline Cytokine & Function & Source & MW (kDa) & Diffusivity $^{*}$ \\
\hline TNF- $\alpha$ & M1 polarization & $\Omega_{\mathrm{H}}$ and $\Omega_{\mathrm{P}}$ & 17 & 1 \\
\hline TGF- $\beta$ & M2 polarization & $\Omega_{\mathrm{H}}$ and $\Omega_{\mathrm{P}}$ & 13 & $3.7606^{*}$ \\
\hline NO & $\begin{array}{l}\text { Triggers tumor } \\
\text { necrosis }\end{array}$ & M1 & & \\
\hline $\begin{array}{l}\text { Ang2/growth } \\
\text { factor }\end{array}$ & $\begin{array}{l}\text { Stimulates } \\
\text { growth/proliferation }\end{array}$ & M2 & 70 & 0.26591 \\
\hline
\end{tabular}

Table 5.1. Macrophage-associated cytokines in the tumor microenvironment (adapted from Mahlbacher et al. [215]). *Diffusivity similar to IL-10, in Mahlbacher [215].

Table 5.2 Tumor parameters and values

\begin{tabular}{|l|l|l|}
\hline Parameter & Value & Reference \\
\hline Hypoxic tissue threshold & 0.5750 & {$[219]$} \\
\hline Necrotic tissue threshold & 0.5325 & {$[219]$} \\
\hline $\mathrm{O}_{2}$ diffusivity & $1^{*}$ & {$[217]$} \\
\hline $\mathrm{O}_{2}$ vascular transfer rate & $5^{*}$ & {$[217]$} \\
\hline Normoxic $\mathrm{O}_{2}$ uptake rate & $1.5^{*}$ & {$[217]$} \\
\hline Hypoxic $\mathrm{O}_{2}$ uptake rate & $1.3^{*}$ & {$[217]$} \\
\hline
\end{tabular}




\begin{tabular}{|l|l|l|}
\hline TME $\mathrm{O}_{2}$ uptake rate & $0.12^{*}$ & {$[217]$} \\
\hline $\mathrm{O}_{2}$ decay rate & $0.35^{*}$ & {$[217]$} \\
\hline
\end{tabular}

Table 5.2. Values for model main parameters (adapted from Mahlbacher et al. [215]). All other tumor parameters are as in Wu et al. [217]

*Non-dimensionalized by $\mathrm{O}_{2}$ diffusivity $\left(1 \times 10^{-5} \mathrm{~cm}^{2} \mathrm{~s}^{-1}\right)$; from Nugent et al. [220]. 
The angiogenesis model $[216,217,221]$ depicts tumor-derived neovascularization, the flow through the network, and both mechanical and chemical effects of tumor growth on various properties of the intrinsic vasculature matrix, simplified as a grid. Nascent angiosprouts are stimulated to form by TAFs produced by tumor tissue, in response to environmental stressors. The model recapitulates the high vascularization of the liver, thereby further enhancing the potential of metastatic seeding and growth.

In keeping with the evolution of the tumor growth with differential oxygenation regions, the vascular growth is therefore randomized in its layout such that the tumor cells have correspondingly heterogeneous potential within the matrix space of having access to nutrients from the vessels. Angiosprouts are produced semi-stochastically, with growth probability weighted by the local hypoxic tissue-generated TAF gradient within the system [216, 221]. Sensitivity to growth of vasculature increases as cytokines diffused by the macrophage populations comes into contact with the vessels [215].

Oxygen transport, $\sigma$, diffuses with coefficient $D_{\sigma}$ from vessel location, and is supplied at rates $\lambda^{\sigma}{ }_{\text {neo }}$ and $\lambda_{\text {pre }}^{\sigma}$ from the neo- and pre-existing vasculature, respectively. Oxygen uptake by normal cells has the rate $\lambda_{\text {tissue }}^{\sigma}$, normoxic tumor cells uptake oxygen with the rate of $\lambda^{\sigma}$ tumor , while hypoxic cells have the rate of $q_{\sigma}$. Oxygen decays with rate $\lambda_{V}^{\sigma}$ in the necrotic region. Oxygen transport is described as [216]: 


$$
\begin{aligned}
& 0=\nabla \cdot\left(D_{\sigma} \nabla \sigma\right)-\lambda^{\sigma}(\sigma) \sigma+\lambda_{e v}^{\sigma}\left(\mathbf{x}, t, \mathbf{1}_{\text {vessel }}, p, \sigma, h\right), \\
& \lambda^{\sigma}=\left\{\begin{array}{ll}
\lambda_{\text {tissue }}^{\sigma} & \text { outside } \Omega \\
\lambda_{\text {tumor }}^{\sigma} & \text { in } \Omega_{P} \\
q_{\sigma}(\sigma) & \text { in } \Omega_{H} \\
\lambda_{N}^{\sigma} & \text { in } \Omega_{N}
\end{array},\right.
\end{aligned}
$$

where $\mathbf{x}$ is position in space, $t$ is time, $\mathbf{1}_{\text {vessel }}$ is the vessel characteristic function (equal to 1 at vessel locations, and 0 otherwise), $p$ is the solid tumor pressure, and $h$ is the hematocrit in the vascular network relating to extravasation of oxygen (following Macklin [216]). The extravasation is modulated by the extravascular interstitial pressure $p_{i}$, scaled by the effective pressure $p_{\mathrm{e}}$, with $k_{P i}$ being the weight of small molecule convective transport components [222]:

$$
\lambda_{e v}^{\sigma}=\bar{\lambda}_{e v}^{\sigma} \mathbf{1}_{v e s s e l}(\mathbf{x}, t)\left(\frac{h}{\bar{H}_{D}}-\bar{h}_{\min }\right)^{+}\left(1-k_{p_{i}} \frac{p_{i}}{p_{e}}\right)(1-\sigma),
$$

where $\lambda^{\sigma}{ }_{e v}$ is the constant oxygen transfer rate from both pre-existing and tumorinduced vessels. Constants $H_{D}$ and $h_{\text {min }}$, respectively, represent normal and minimum blood hematocrit required for oxygen extravasation. Oxygen values are normalized with respect to the vasculature, ranging from 0 to 1.

Following Mahlbacher et al and Leonard et al. [215, 223], monocytes are simulated to extravasate from the vasculature in proportion to the local concentration gradient of macrophage chemoattractants (e.g., TAFs produced by hypoxic tumor tissue), and to preferentially migrate towards tissue regions (e.g., hypoxic tissue) along the increasing chemoattractant gradient. Monocytes 
polarize to M1 or M2 subtypes in the local TME according to the cytokine concentration release from proliferating normoxic and non-proliferating, nutrientseeking hypoxic tumor cells (see Table 5.3 below). Monocytes and macrophages are simulated as discrete entities using a cellular automaton algorithm.

With the number of cancer cells correlating to size of tumor mass, a $1 \mathrm{~mm}^{3}$ tumor can be estimated to contain up to $3 \times 10^{6}$ cells; approximately $10 \%$ of this total is estimated to be macrophages. A conservative estimate, then, for the number of macrophages recruited by the tumor is $\sim 25 \%$ of the reported amount observed in liver tumor metastases in vivo $\left(\sim 2.78 \times 10^{4}\right.$ macrophages $/ \mathrm{mm}^{3}$; [219]).

In simulations, M1 macrophages were calibrated to recapitulate data indicating their deeper penetration into tumor mass than M2 subtypes; this was modeled as a concentric field of a value of 1 at the center of tumor mass, and a value of 0 at the tumor boundary [223]. Thus, the model is biased to direct M1 macrophage movement based on its distance from the center of the tumor lesion.

Table 5.3. Macrophage model associated parameters

\begin{tabular}{|c|c|c|c|}
\hline Parameter & Description & Value & Reference \\
\hline \multicolumn{4}{|c|}{ Physiological parameters } \\
\hline Macrophage mass & $\begin{array}{l}\% \text { of macrophages } \\
\text { per tumor mass }\end{array}$ & $10 \%$ & [219] \\
\hline M2/M1 ratio (hi-met) & $\begin{array}{l}\text { Highly-metastatic } \\
\text { tumors }\end{array}$ & 2.06 & [224] \\
\hline M2/M1 ratio (norm) & $\begin{array}{l}\text { Moderately- } \\
\text { metastatic tumors }\end{array}$ & 0.77 & [224] \\
\hline \multicolumn{4}{|c|}{ Vessel Radius-associated parameters } \\
\hline $\begin{array}{l}\text { Host vessel grid } \\
\left(1^{\circ} \text { tumors) }\right.\end{array}$ & $\begin{array}{l}\text { Initial vascular } \\
\text { density }\end{array}$ & $8 \times 8$ & -- \\
\hline $\begin{array}{l}\text { Host vessel grid } \\
\left(2^{\circ} \text { tumors) }\right.\end{array}$ & $\begin{array}{l}\text { Initial vascular } \\
\text { density }\end{array}$ & $19 \times 19$ & -- \\
\hline
\end{tabular}




\begin{tabular}{|c|c|c|c|}
\hline$\lambda_{M}$ & $\begin{array}{l}\text { Tumor native } \\
\text { proliferation rate } \\
\left(\text { day }^{-1}\right)\end{array}$ & 0.5 & [219] \\
\hline$\lambda_{O L}$ & $\begin{array}{l}\text { Recovery rate of } \\
\text { quiescent oxygen } \\
\text { level }\end{array}$ & $0.05^{*}$ & [223] \\
\hline$\lambda_{O T}$ & $\begin{array}{l}\mathrm{M} 2 \text { induced } \\
\text { lowering viable } \mathrm{O}_{2} \\
\text { threshold rate }\end{array}$ & $200 / s$ & [223] \\
\hline$\lambda_{\text {rec }}$ & $\begin{array}{l}\text { Recovery rate of } \\
\lambda_{M 2} \text { to zero }\end{array}$ & $0.1^{*}$ & [223] \\
\hline$\lambda_{F}$ & $\begin{array}{l}\text { M2 induced } \\
\text { proliferation rate }\end{array}$ & $1000 / \mathrm{s}$ & [223] \\
\hline$\lambda_{N O}$ & $\begin{array}{l}\text { M1 nitric oxide } \\
\text { induced death rate }\end{array}$ & $3 / \mathrm{s}$ & [225] \\
\hline$G_{N}$ & $\begin{array}{l}\text { Cell degradation } \\
\text { rate in the necrotic } \\
\text { region }\end{array}$ & $0.3^{*}$ & [219] \\
\hline \multicolumn{4}{|c|}{ Macrophage Differentiation Scaling Coefficients } \\
\hline$k_{M 1}$ & M1 macrophage & 200 & [223] \\
\hline$k_{M 2}$ & M2 macrophage & 20 & [223] \\
\hline \multicolumn{4}{|c|}{ Macrophage Movement Scaling Coefficients } \\
\hline$M_{O}$ & $\begin{array}{l}\text { Effect of oxygen on } \\
\text { macrophage } \\
\text { movement }\end{array}$ & 1000 & [219] \\
\hline$M_{P}$ & $\begin{array}{l}\text { Effect of oxygen on } \\
\text { macrophage } \\
\text { movement }\end{array}$ & 350 & [219] \\
\hline$M_{C}$ & $\begin{array}{l}\text { Chemotactic } \\
\text { macrophage } \\
\text { movement }\end{array}$ & 500 & [219] \\
\hline \multicolumn{4}{|c|}{ ECM Turnover Parameters } \\
\hline $\begin{array}{l}\text { ECM production } \\
\text { constant }\end{array}$ & CECM & 5 & $\begin{array}{l}\text { Calibrated from } \\
\text { matrisome analysis } \\
\text { [45] }\end{array}$ \\
\hline $\begin{array}{l}\text { ECM degradation } \\
\text { constant }\end{array}$ & cECM & 1 & $\begin{array}{l}\text { Calibrated from } \\
\text { matrisome analysis } \\
\text { [45] }\end{array}$ \\
\hline $\begin{array}{l}\text { ECM production } \\
\text { constant }\end{array}$ & tECM & 10 & $\begin{array}{l}\text { Calibrated from } \\
\text { matrisome analysis } \\
\text { [45] }\end{array}$ \\
\hline $\begin{array}{l}\text { ECM degradation } \\
\text { constant }\end{array}$ & tECM & 0.1 & $\begin{array}{l}\text { Calibrated from } \\
\text { matrisome analysis } \\
\text { [45] }\end{array}$ \\
\hline
\end{tabular}


Table 5.3. Characteristics of macrophage model parameters (adapted from Mahlbacher et al. [215]). *Value non-dimensionalized by $\mathrm{O}_{2}$ diffusivity $\left(1 \times 10^{-5}\right.$ $\mathrm{cm}^{2} \mathrm{~s}^{-1}$; Nugent et al. [220]).

Following calibration of tumor, vascular, and macrophage protein parameters, the effects of ECM and ECM-macrophage population interactions on tumor growth were evaluated in various cases, as stated below:

Table 5.4. Macrophage model simulation conditions

\begin{tabular}{|c|c|c|c|c|}
\hline Control Simulations & Case 1 & Case 2 & Case 3 & Case 4 \\
\hline M1 cytokine & & & & \\
\hline M2 cytokine & & & & \\
\hline ECM comp & cECM & tECM & cECM & tECM \\
\hline Tumor Type & Primary & Primary & Metastatic & Metastatic \\
\hline Experimental Simulations & Case 1 & Case 2 & Case 3 & Case 4 \\
\hline M1 cytokine & M1 & M1 & M1 & M1 \\
\hline M2 cytokine & M2 & M2 & M2 & M2 \\
\hline ECM comp & cECM & tECM & cECM & tECM \\
\hline Tumor Type & Primary & Primary & Metastatic & Metastatic \\
\hline
\end{tabular}

Table 5.4. Model conditions for simulation experiments. The control simulation cases were run first to assess tumor response to differential ECM composition. The other cases then explored the response to heterogeneous macrophage populations. The simulation timespan for each case was 13 days.

The effects of the M1 and M2 macrophage subtypes are quantified via secretion of nitric oxide (NO) and tumor growth factors, respectively. Thus, M2 macrophages favor tumor growth, manifested as the lowering of the hypoxic 
tissue threshold; M1 macrophages oppose this effect via secretion of NO, leading to tumoricidal activity. These effects, termed $\lambda_{M 1}$ and $\lambda_{M 2}$, have been included in the proliferation term below [215]:

$\lambda_{p}=\left\{\begin{array}{lr}\text { non tumoral }: & 0 \\ \Omega_{P}: & \left(\lambda_{M}+\lambda_{M 2}\right) \sigma-\left(\lambda_{A}+\lambda_{M 1}\right) \\ \Omega_{H}: & \lambda_{M 2} \sigma-\left(\lambda_{A}+\lambda_{M 1}\right) \\ \Omega_{N}: & -G_{N}\end{array}\right.$,

where $\lambda_{M}$ is the tumor native mitosis rate, $\sigma$ is the local oxygen concentration calculated by Equation 3 , and $\lambda_{A}$ is the baseline apoptosis rate. The nondimensionalized cell degradation rate of the necrotic region is $G_{N}$; this rate assumes the constant degradation and removal of necrotic cellular debris and associated fluid. M1 cytotoxicity is modeled to affect both proliferating (cycling) and hypoxic (quiescent) tissue, since cell death presumed to be independent of cellcycle mechanistics.

The antitumoral effect, $\lambda_{M 1}$ of the M1 subtype is simulated to affect tissue proportional to the release rate $\lambda_{N O}$, of nitric oxide in the immediate vicinity of the macrophage $\left(\mathbf{1}_{M \mathbf{1}}\right)$, since nitric oxide has a short half-life in vivo with limited diffusion distance [215].

$$
\lambda_{M 1}=\lambda_{N O} \mathbf{1}_{M 1}
$$

M2 growth factor, in addition inhibition of tumor cytotoxicity, positively affects the proliferating region as follows [215]: 
$\frac{d \lambda_{M 2}}{d t}=\lambda_{F} F\left(1-\left(\lambda_{M}+\lambda_{M 2}\right)\right)$

where $\lambda_{M 2}$ is the proliferation rate related to the concentration, $F$, of diffusible M2 growth factor; this adds to the native proliferation rate $\lambda_{M}$. The effect of the M2 growth factor on tumor proliferation is $\lambda_{F}$. Thus, the proliferation effect due to $\lambda_{M 2}$ actually decreases as net proliferation $\left(\lambda_{M}+\lambda_{M 2}\right)$ approaches the maximum value of 1 day $^{-1}$.

M2 macrophages can also stimulate the proliferation of quiescent, hypoxic tumor cells, though this occurs at lower rates than in normoxic, actively proliferating tissue. The M2 macrophage-secreted tumor growth factor concentration, $F$, can transiently lower the local viable oxygen threshold as follows [215]:

$\frac{d Q_{O L}}{d t}=\lambda_{O L} \cdot(1-F) \cdot\left(\bar{Q}_{O L}-Q_{O L, \text { current }}\right)-\lambda_{O T} \cdot F \cdot\left(Q_{O L, \text { current }}-Q_{O L, \text { min }}\right),[9]$

where $Q_{O L}$ is the quiescence oxygen level, $\lambda_{O L}$ is the quiescence oxygen level recovery rate back to the standard quiescence oxygen level, $\bar{Q}_{O L}, Q_{O L, c u r r e n t}$ is the current quiescence oxygen level, $F$ is the local concentration of M2 growth factor ([0,1]; dimensionless units), $\lambda_{O T}$ is the $\mathrm{M} 2$ growth factor effect rate on the lowering of the viable oxygen threshold, and $Q_{O L \min }$ is the lower bound of the quiescence oxygen level. Effective oxygen levels are set to $\bar{Q}_{O L}$ if they exceed $\bar{Q}_{O L}$, and to $Q_{O L \min }$ if less than $Q_{O L \min }$. 
As monocytic precursors, $M \phi$, extravasate from the tumor-adjacent capillaries, they encounter proteins diffused tumor interior and vasculature that induce differentiation to macrophages. The concentration of factors, which correlate to interleukins and other cytokines that encourage differentiation and subsequent polarization to macrophage population subtypes, influences the respective differentiation rate, $R_{i}$.

Under steady-state conditions, the overall mass balance for any cytokine concentration, $C$ (dimensionless units), produced by the viable (either proliferating or hypoxic) tumor regions is [226]:

$0=\nabla \cdot\left(D_{C} \nabla C\right)+\bar{\lambda}_{\text {production }}^{C}(1-C) \mathbf{1}_{\Omega}-\bar{\lambda}_{\text {circulation }}^{C} \mathbf{1}_{\text {vessel }}-\bar{\lambda}_{\text {decay }}^{C} C$,

where $D_{C}$ is diffusivity and $\bar{\lambda}_{\text {production }}^{C}, \bar{\lambda}_{\text {circulation }}^{C}$, and $\bar{\lambda}_{\text {decay }}^{C}$ are constant, nondimensional rates of cytokine production, circulation washout, and decay, respectively. These concentration values range from 0 to 1 . Zero Neumann conditions are taken at the boundaries for all diffusion equations [216].

Oxygen, pressure, and chemoattractant gradients direct both monocyte and macrophage migration through the interstitium. Movement in one of four directions along the computational scaffold grid is governed semi-stochastically.

The values of macrophage-associated parameters are defined in Table 5.3. Parameter values were set as in Mahlbacher et al, according to published literature, or otherwise calibrated to correlate simulated tumor growth to experimental endpoints. Cytokine characteristics are summarized in Table 5.1, 
based on prior work which classified protein diffusivity relative to molecular weight [226]. Wash-out rate into the vasculature, decay rate, diffusivity, and production rates for each cytokine can be found in Table 5.5.

Table 5.5. Macrophage cytokine rate parameters

\begin{tabular}{|l|l|l|}
\hline Parameter & Function & Value \\
\hline $\bar{\lambda}_{\text {circulation }}^{C}$ & $\begin{array}{l}\text { Wash-out rate into } \\
\text { vasculature }\end{array}$ & $0.006^{*}$ \\
\hline $\bar{\lambda}_{\text {decay }}^{C}$ & Decay rate & $0.001^{*}$ \\
\hline$D_{M 1 f}$ & Diffusivity for $M 1 f$ & $0.005^{*}$ \\
\hline$D_{M 2 f}$ & Diffusivity for $M 2 f$ & $0.01880^{*}$ \\
\hline$D_{I L-10}$ & Diffusivity for $I L-10$ & $0.01880^{*}$ \\
\hline$D_{T 2 f}$ & Diffusivity for T2f & $0.005^{*}$ \\
\hline$D_{\text {Ang } 2}$ & Diffusivity for Ang2 & $0.00133^{*}$ \\
\hline $\bar{\lambda}_{\text {production }}^{M 1 f}$ & production rate of $M 1 f$ & $1.0^{* *}$ \\
\hline $\bar{\lambda}_{\text {production }}^{M 2 f}$ & production rate of $M 2 f$ & $1.0^{* *}$ \\
\hline $\bar{\lambda}_{\text {production }}^{I L-10}$ & production rate of $I L-10$ & $1.0^{* *}$ \\
\hline $\bar{\lambda}_{\text {production }}^{T 2 f}$ & production rate of $T 2 f$ & $1.0^{* *}$ \\
\hline $\bar{\lambda}_{\text {production }}^{\text {Ang }}$ & production rate of Ang2 & $1.0^{* *}$ \\
\hline
\end{tabular}

Table 5.5. Model rate parameters for macrophage-associated cytokines adapted from Mahlbacher et al. [215]. Washout and decay rates are generically applied to all cytokines, C. (Based on proteomic analysis in Frieboes et al. [226]).

*Value non-dimensionalized by $\mathrm{O}_{2}$ diffusivity $\left(1 \times 10^{-5} \mathrm{~cm}^{2} \mathrm{~s}^{-1}\right.$; Nugent et al. [220]). ${ }^{* *}$ Value rescaled by the production rate of VEGF-A (VEGF -165) protein, for a general TAF molecule.

\section{Results}

1. Validation of liver decellularization 
Tissues were evaluated both on gross pathology and via histological analysis to confirm decellularization of liver tissue (Figure 5.1). Following $48 \mathrm{~h}$ decellularization, liver "ghosts" visually showed translucence of gross tissue and were sufficiently acellular to continue with lyophilization step. 


\section{Figure 5.1. SDS decellularization.}

Gross pathology and histological analysis of decellularized liver tissues. (A): Liver tissues at each step of decellularization; at $48 \mathrm{~h}$, incubation in SDS solution yields translucent tissue "ghost" prepared for lyophilization. (B): Histological analysis of decellularized liver tissues. Top row shows control livers; bottom row represents acellular samples. In the first column, Sirius Red/Fast Green collagen staining shows normal tissue in top row, with red staining of collagen, reticulin fibers, and basement membrane, and green staining of non-collagenous proteins, while the decellularized sample in bottom row shows only collagenous material remaining. The second column shows H\&E stain of normal tissue (top), and denucleation of acellular samples in the bottom row. The third column shows Trichrome stain of normal tissue (top), with nuclei stained in black, cytoplasm stained red, and collagen in blue; the acellular sample (bottom) shows removal of nuclei and cytoplasm, with retention of collagen in blue. 
A.

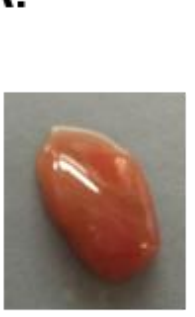

SDS Decellularization

$$
\mathrm{T}=0
$$
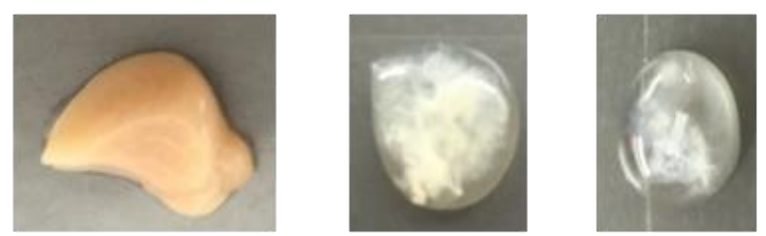

O/N PBS Wash

$24 \mathrm{~h}$ SDS

$48 \mathrm{~h}$ SDS

B.

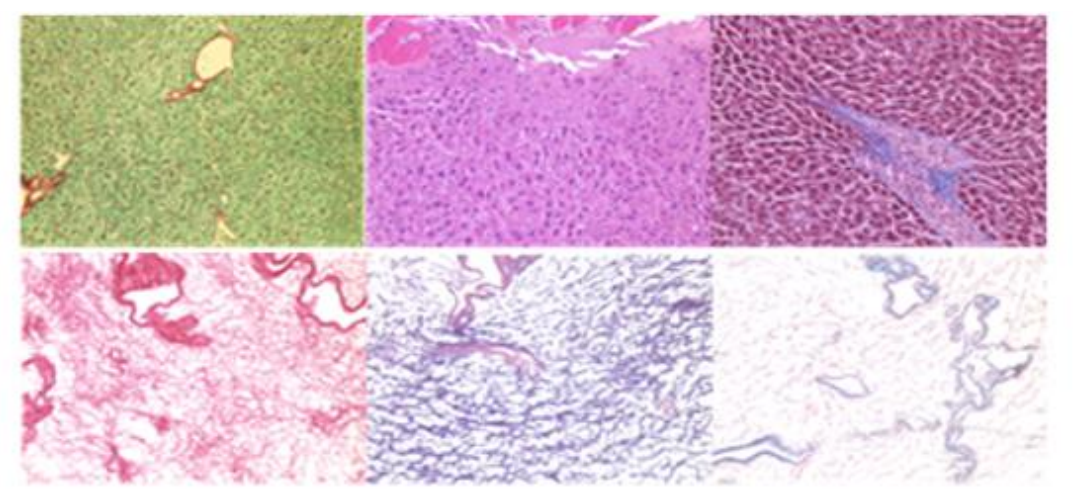




\section{Scheme 5.2. Tumor transwell migration assay.}

Primary and metastatic tumor cell lines were seeded on transwells with $8 \mu \mathrm{m}$ porous membrane in chamber insert, coated with either cECM or tECM LECM liver ghosts. Receiver plates contained media +/ FBS serum and cells were allowed to migrate for 48 hours prior to fixation and staining of membrane to determine relative quantities of cells that had passed through the membrane. 

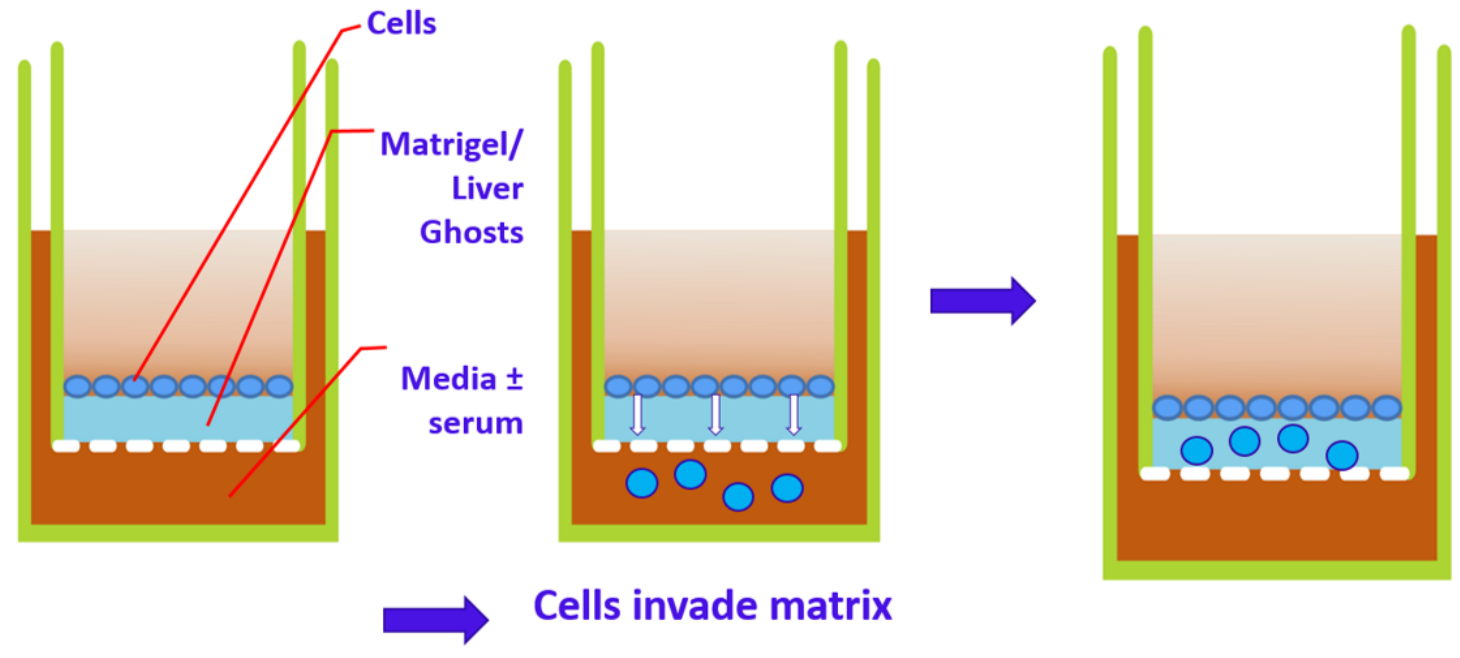

Cells on membrane Fixed \& stained

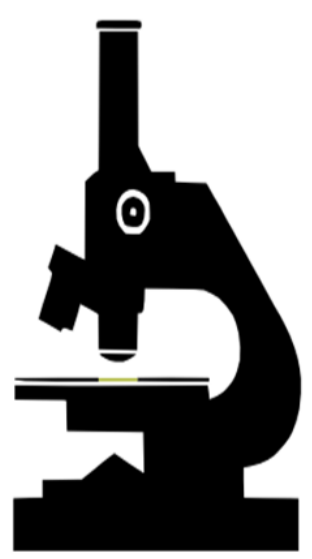

Cells counted 
Figure 5.2. Tumor transwell migration cell counts.

Transwell membranes fixed and stained with crystal violet were assessed for quantification of migrated tumor cells. Five separate fields per slide were counted and averaged. Serum-free media controls were used to establish baseline migration with no chemoattractant in receiver well; ECM controls with no LECM treatment were utilized as well. Both primary and metastatic tumors plated on tECM with serum supplement exhibited increased migration as compared to all other experimental groups $(P<0.05)$. 


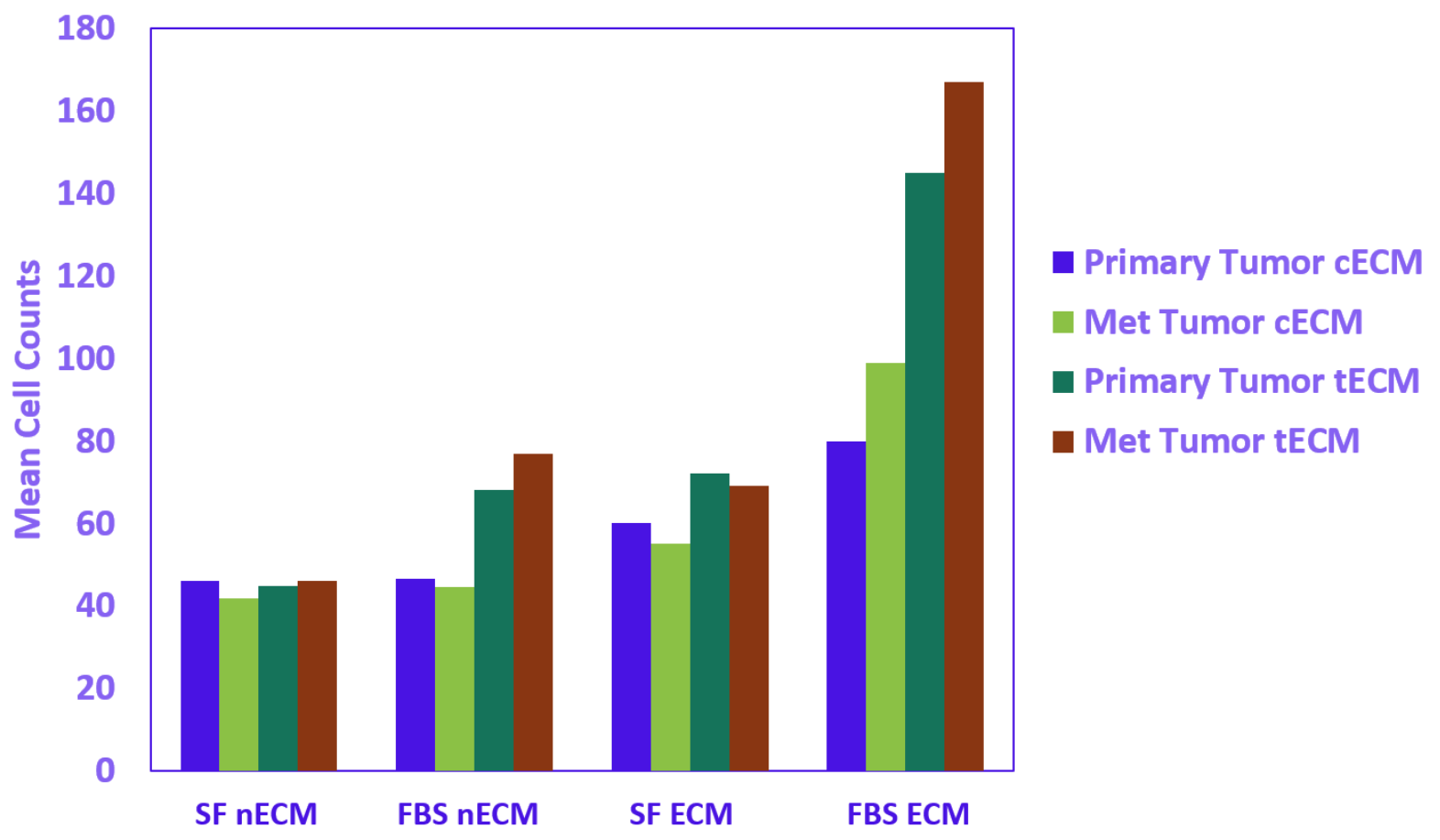




\section{Scheme 5.3. Indirect co-culture assay.}

Indirect co-culture conditions. Naïve macrophages were seeded in bottom receiver plate well at a density of $1 \times 10^{5}$ cells/well. Control macrophages remained naïve and unactivated, while LPS and IL-4 treatments induced polarization to M1 and M2 activation states, respectively. Transwells with $0.4 \mu \mathrm{m}$ membranes were utilized to prevent migration of tumor cells from top of transwell chamber to receiving plate below. Either primary or metastatic tumor cell lines were seeded in transwell insert at $5 \times 10^{4}$ cells/well. After $48 \mathrm{~h}$, supernatant was harvested for ELISA analysis. This assay allows for tumor and macrophage cell populations to influence each other, with free exchange of cytokines across the transwell membrane, while restricting direct cell-cell contact between the two cultures. 


\section{Macrophage-Tumor Cell Indirect Co-culture}

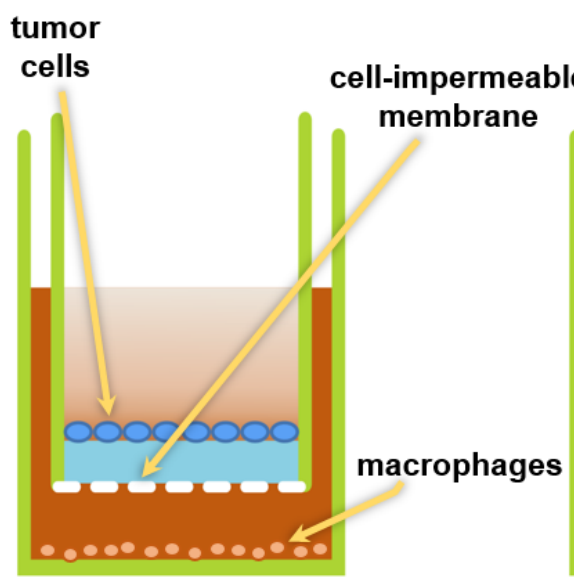

Control

Naïve MФ

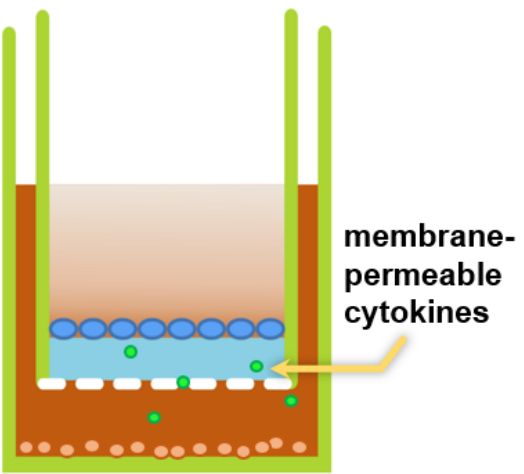

LPS-activated M1 macrophages

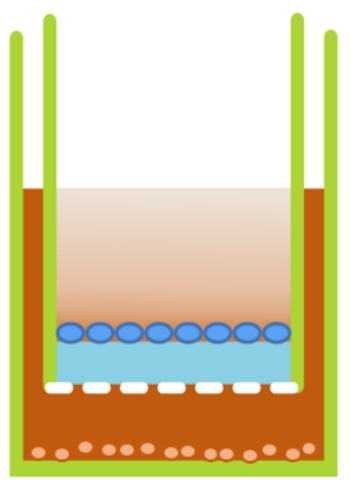

IL-4-activated M2 Macrophages 
2. ELISA analysis of TGF $\beta-1$ and TNF- $\alpha$ present in co-culture system Data from ELISA assay was analyzed using two-way ANOVA with Bonferroni posttests to compare replicate means with regard to cell line. ELISA results indicated that while TNF- $\alpha$ levels were significantly higher in the M1activated macrophage group for each cell line, the metastatic tumor cell line exhibited the highest induction of TNF- $\alpha$ at $329.96 \pm 18.89(P<0.001$; Figure 5.3) . TGF $\beta-1$ levels were less dynamic, with only the primary tumor cell line exhibiting a significant difference between M1- and M2-activated macrophages $(P<0.05)$. These levels were then utilized for calibration of macrophage proteins in tumor model. 


\section{Figure 5.3. TNF- $\alpha$ and TGF $\beta-1$ ELISAs.}

ELISA results from indirect co-culture assay. Control transwells that were established with only macrophages seeded to the receiver plate without tumor cells in the insert showed no differences in TGF $\beta-1$ levels across the treatment groups. The TNF- $\alpha$ levels indicated a significant difference between the control macrophages and the M1-activated macrophages $(P<0.01)$. In transwells seeded with primary tumor cells in the inserts, TGF $\beta-1$ was significantly higher in the M1activated macrophage group than the M2-activated macrophages. TNF- $\alpha$ levels in the primary tumor group were significantly increased among M1-activated macrophages compared to M2-activated and naïve macrophages $(P<0.01$ and $P<0.05$, respectively). In transwells seeded with metastatic tumor cells in the inserts, there were no significant differences in TGF $\beta-1$ levels. TNF- $\alpha$ levels in this group were significantly increased in M1-activated macrophages compared to M2-activated and control macrophages $(P<0.001)$. Additionally, TNF- $\alpha$ differed significantly between the control and M2 groups $(P<0.01)$. 


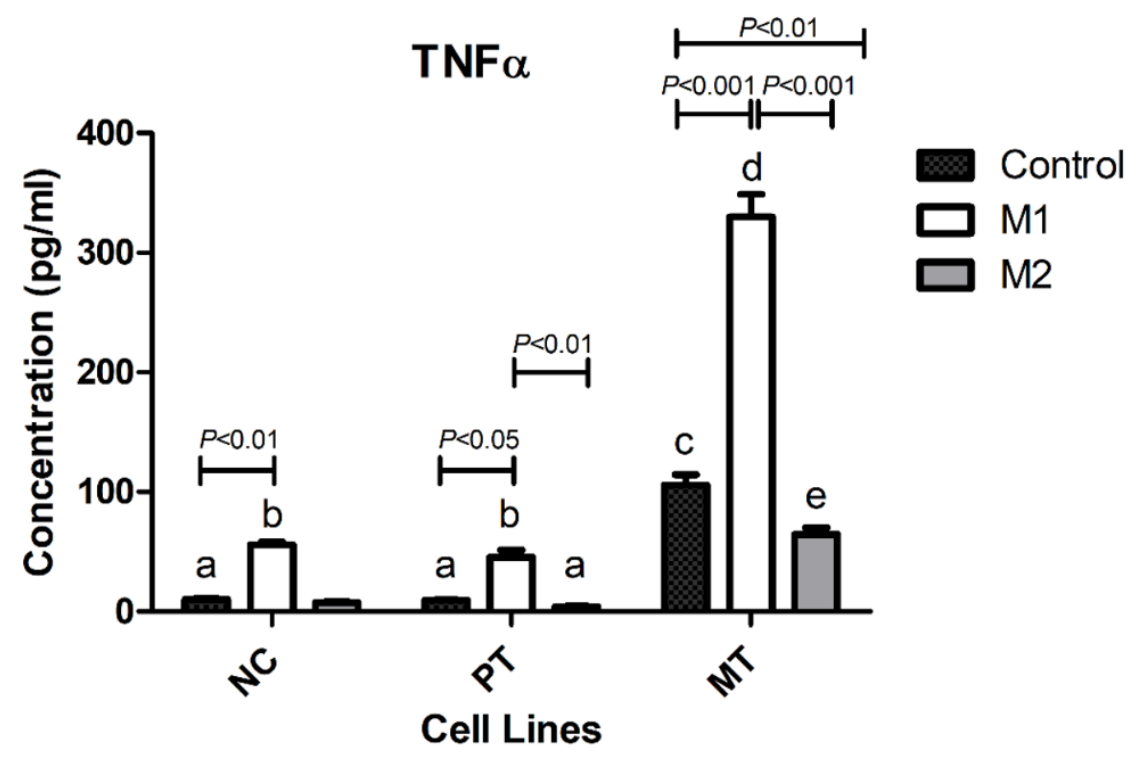

TGF $\beta 1$

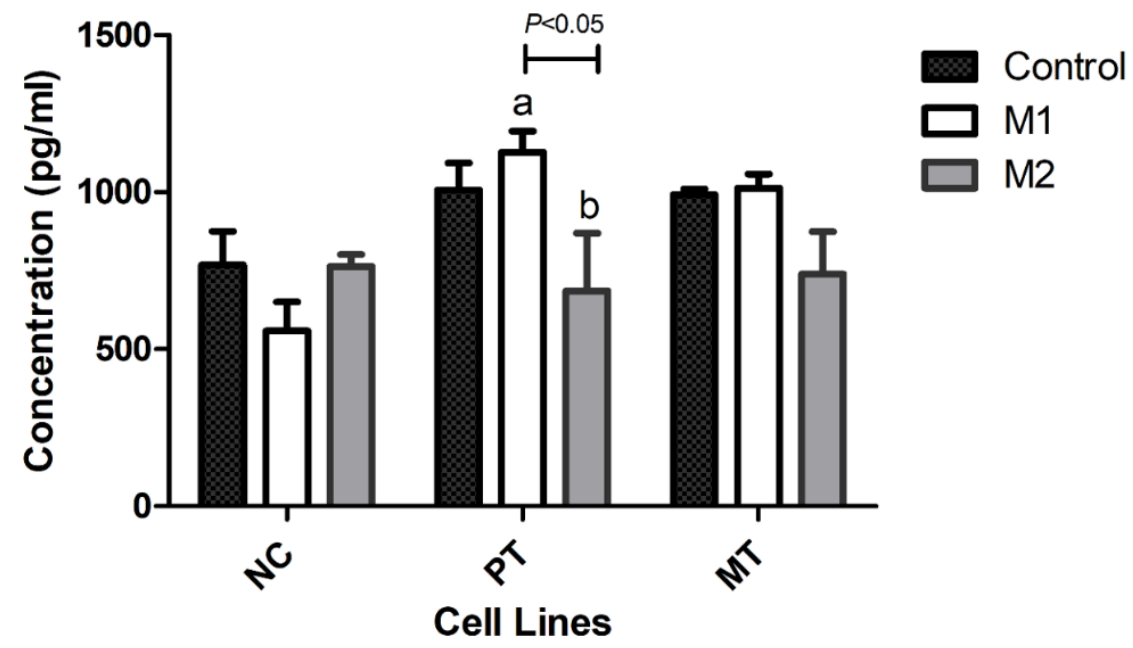


3. Visualization of tumor growth simulations

Data from transwell migration assay was used to calibrate ECM degradation/production ratio in model in the absence of macrophages (Table 5.3; Figure 5.4). This control simulation was run with only monocyte populations, to assess the effects of ECM modulations alone. The vascular grid for metastatic tumors was set to a higher density than that for the primary tumors $(19 \times 19 \mathrm{vs}$. $8 \times 8$, respectively). This is reflected in the increased grid density on the uppermost left-hand corners of each $3 \times 3$ output grid from the simulations. For the primary tumors, there are larger regions of necrotic tissue (in brown; Figure 5.4); the metastatic tumors show blue hypoxic cores. As shown in the bottom leftmost corner panel of each grid, the ECM calibration has resulted in both a higher ECM density for the tECM simulations relative to the $\mathrm{CECM}$, and a higher ECM density for metastatic tumors respective to the primary tumor simulations.

For the experimental simulations, the same calibrations were employed as above, with the addition of the TNF- $\alpha$ and TGF $\beta-1$ macrophage chemoattractants, as correlated from data from the indirect co-culture ELISA assay. The primary tumor simulations produced a more even distribution of M1 and M2 subtypes, while the metastatic tumors had a higher concentration of M1 macrophages that were infiltrating the tumor mass, following the gradient of macrophage chemoattractants. The metastatic, $\mathrm{CECM}$ case exhibited monocytes distinctly clustered around tumor mass, with the highest ratio of proliferative cells than all other cases. 
Figure 5.4. ECM-dependent simulations (monocyte populations only). Tumor growth at 13 days with ECM degradation/production ratio was calibrated to simulate murine hepatic control ECM (cECM) and the transitional ECM (tECM) induced by alcohol exposure. The output matrix shows primary and metastatic tumor cells in the top and bottom rows, respectively; cECM and tECM simulations are shown in the first and second columns, respectively. Each $3 \times 3$ grid, comprised of nine $4 \mathrm{~mm}^{2}$ panels, shows the tumor growth and vessels in the top left corner, with proliferating regions in red, quiescent hypoxic regions in blue, and necrotic areas in brown. Both primary tumor simulations in the top row have larger areas of necrosis than the metastatic tumor simulations in the bottom row, which have mostly hypoxic blue cores. The vasculature grid for the metastatic cells was calibrated to be denser, to recapitulate the density of liver vasculature for the distal metastatic site. In the middle and right corners of the top row, the macrophage chemoattractants, TNF- $\alpha$ and TGF $\beta-1$, are shown in heat map as they are secreted from hypoxic tumor tissue. The middle leftmost panel of each grid shows the extravasated monocytes emanating from the vasculature. The middle center and rightmost panel of each grid show the density of Type 1 (M1) and Type 2 (M2) macrophages, respectively, in the tumor microenvironment. As seen in the lower left corner of each grid, the ECM density is higher in the tECM simulations, successfully recapitulating the transitional ECM determined via matrisome analysis for alcohol-exposed liver tissue [45]. The bottom middle and leftmost panels of each grid show tumor oxygenation and tumor angiogenic factor, respectively. 


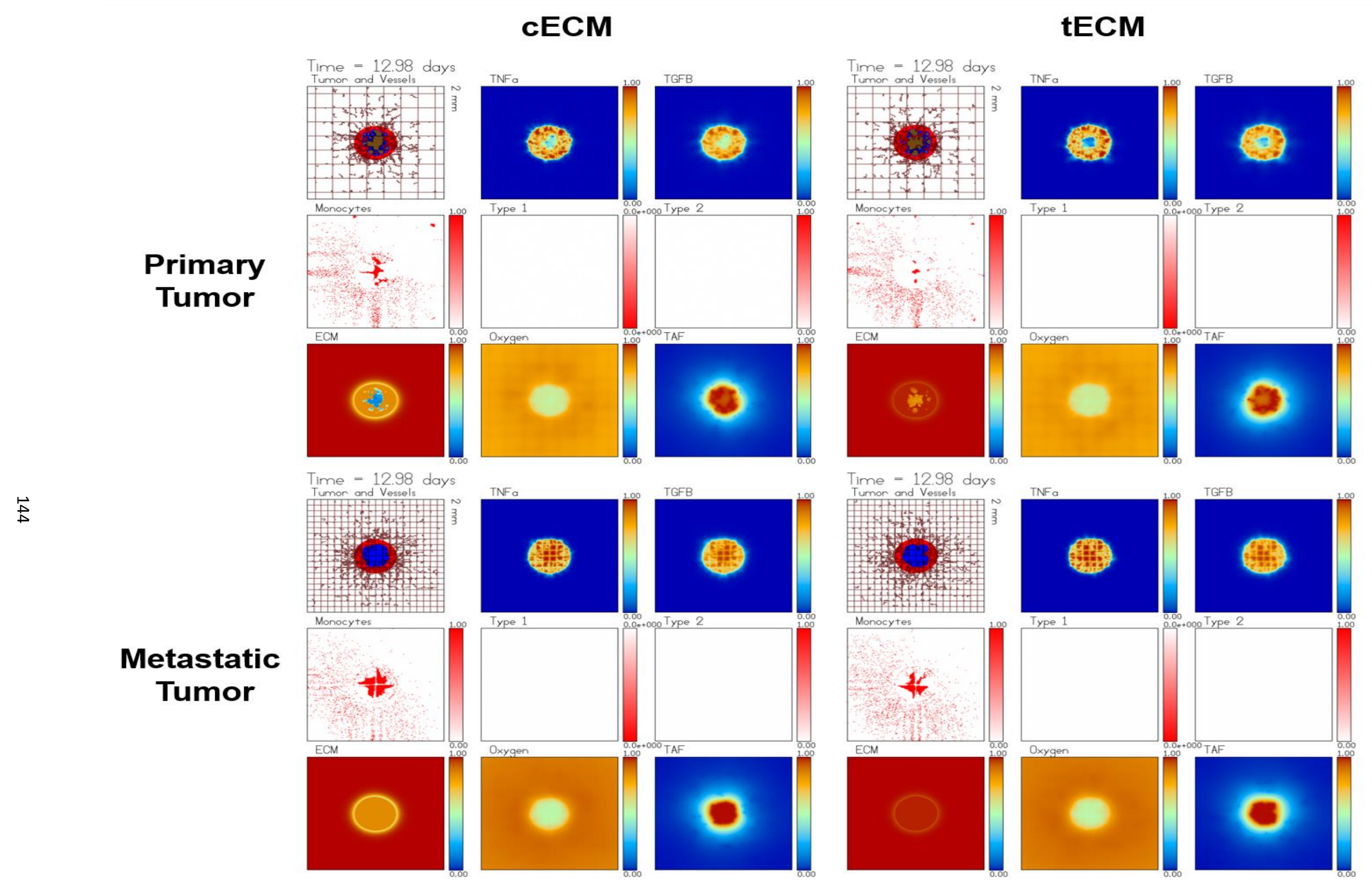




\section{Figure 5.5. ECM-macrophage simulations.}

Tumor growth at 13 days with mixed M1/M2 macrophage subtypes (same grid panel descriptions as in Figure 5.4). The primary tumor cell grids in the top row show tend to ward a balance between M1 and M2 populations, as indicated in the middle row of each $3 \times 3$ grid. Both subtypes appear to have penetrated the tumor mass, and the tumor on the control ECM is slightly larger than the tumor on transitional ECM, as measured by its final tumor radius. The metastatic tumors have a higher infiltration of M1 subtypes, which resulted in growth restriction relative to all other cases. The tumors were less oxygenated and the tECM simulation produced a larger metastatic tumor radius than on $\mathrm{CECM}$. 


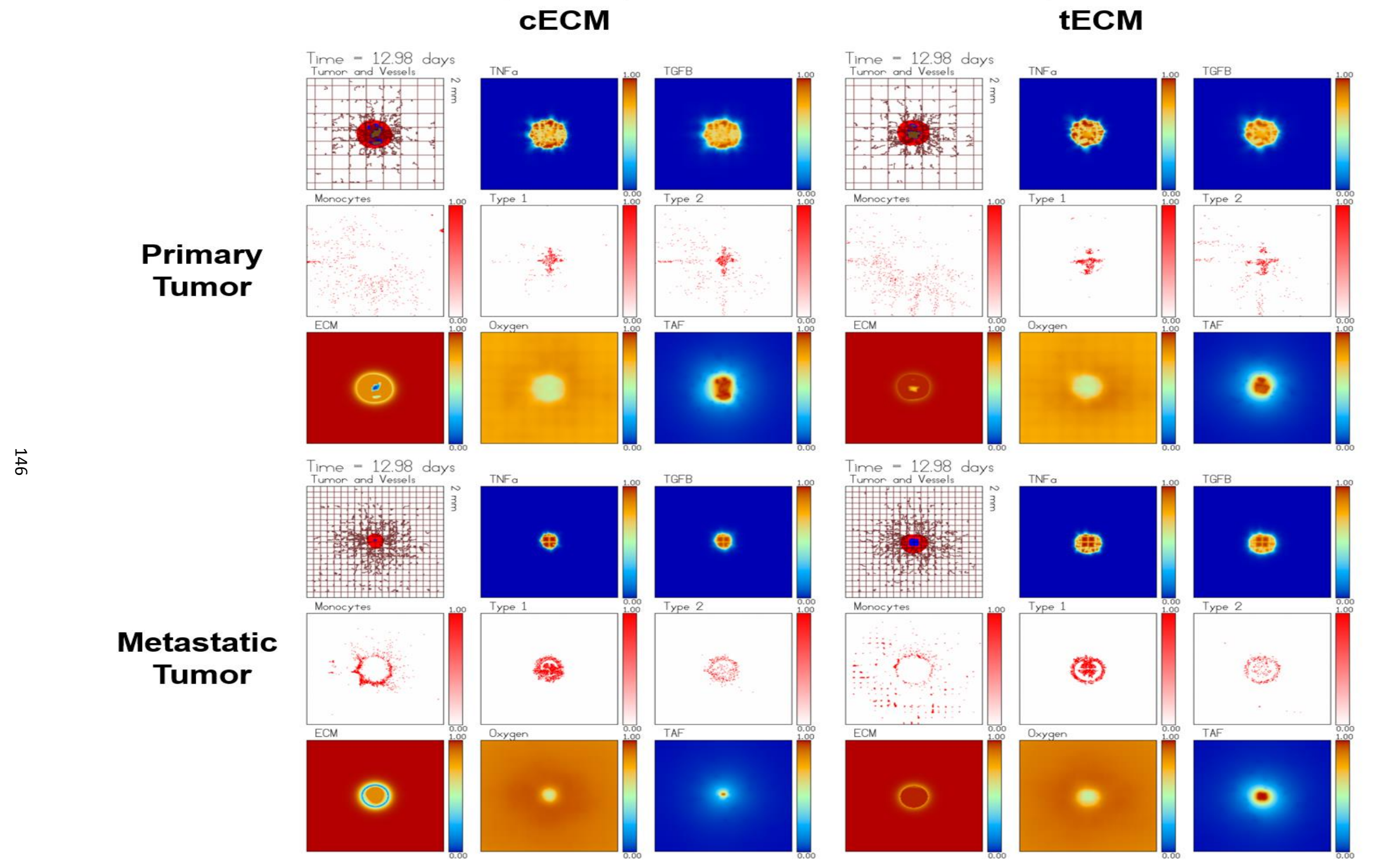


Figure 5.6. Simulation analyses of tumor radii and vessel surface area.

Tumor radius and vessel surface area curves. (A): Tumor radius over $13 \mathrm{~d}$ simulation timespan $(\mathrm{mm})$. Overall, control simulations produced larger tumor masses than experimental simulations. (B): Surface area $\left(\mu \mathrm{m}^{2}\right)$ of tumor vasculature, calculated utilizing capillary radii, assuming individual capillary diameter of $10 \mu \mathrm{m}$. (C): Final tumor radius $(\mathrm{mm})$ for all simulation cases. Among the experimental group, the metastatic tumor on tECM had a larger final tumor radius than the metastatic tumor on control ECM. There were nominal differences in tumor mass among the control simulation group. 
A.

Tumor Radius

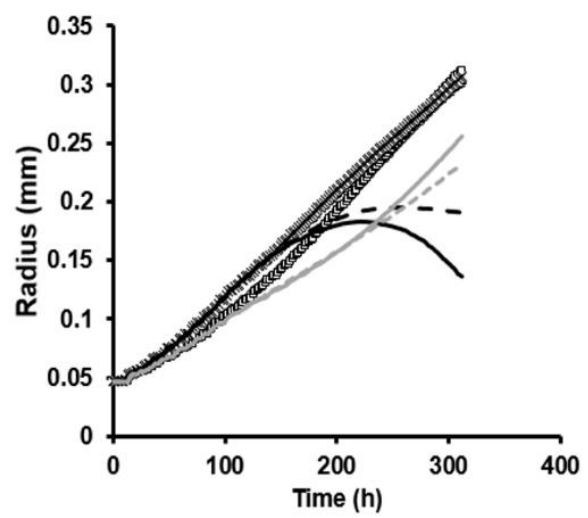

B.

Tumor Vessel Surface Area

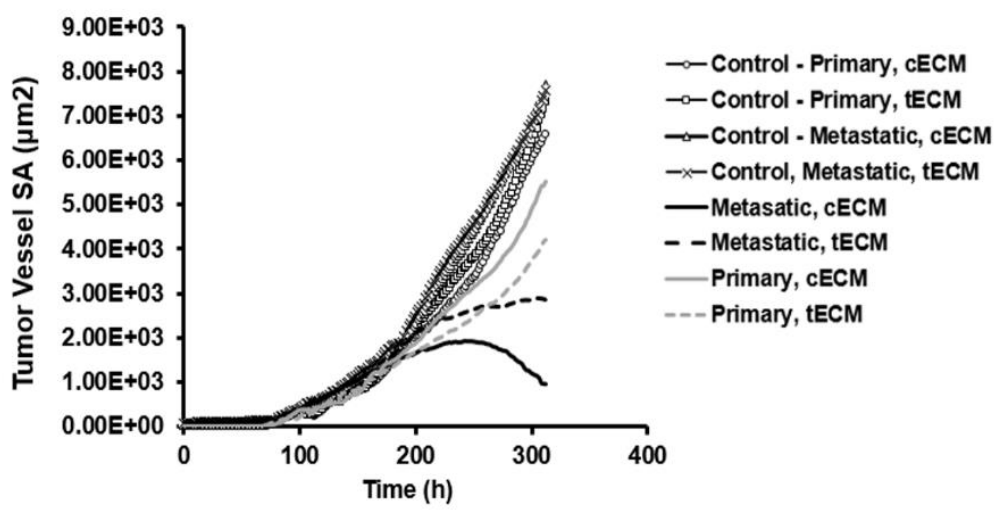

Final Tumor Radius

c.

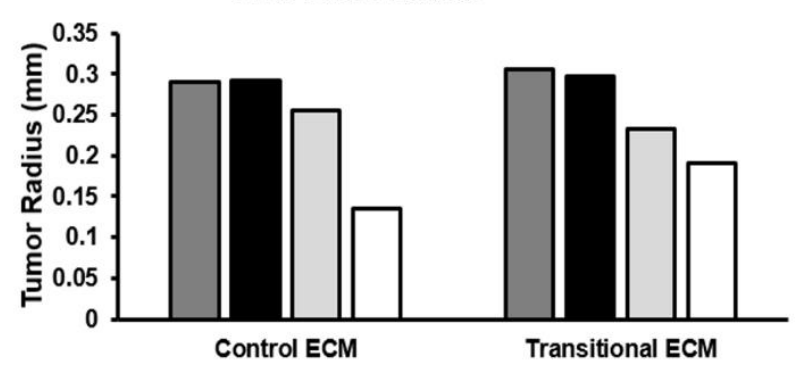

$\square$ (Control) Primary Tumor -(Control) Metastatic Tumor 口(M1 + M2) Primary Tumor 口(M1 + M2) Metastatic Tumor 
Figure 5.7. Macrophage cell counts and population fractions.

Time evolution of macrophage subtype populations. Primary tumor simulations run on either ECM construct exhibited more balanced proportion of M1:M2 macrophage subtypes than metastatic tumors; on either ECM construct, M2 macrophages are more prominent than $\mathrm{M} 1$ for primary tumors. M1 macrophages dominate for the metastatic tumors, recapitulating results from indirect co-culture assay. However, on tECM, a higher proportion of monocytes have differentiated and polarized to $\mathrm{M} 1$ by the end of the simulation than on CECM. 


\section{Macrophage Cell Counts and Population Fractions on Control ECM}

Primary Tumor
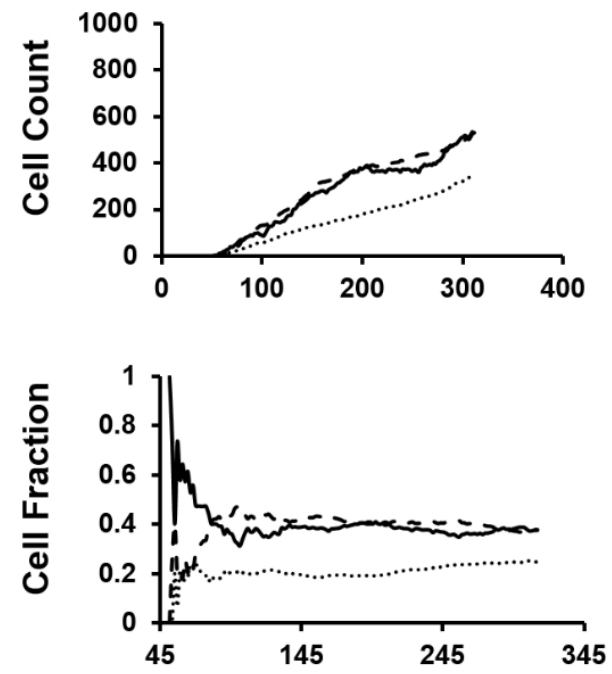

Time (h)
Metastatic Tumor
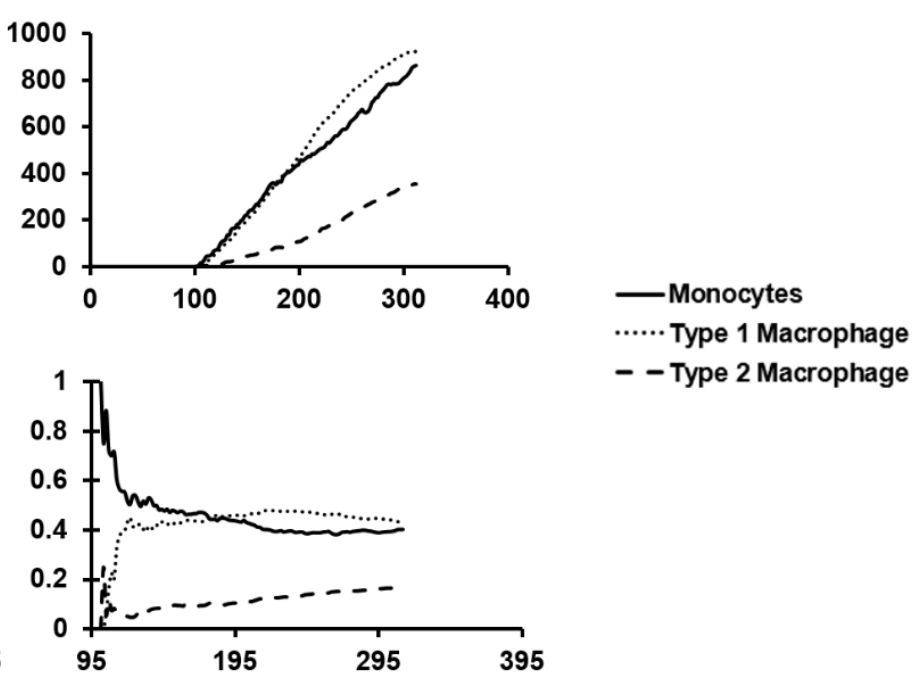

- - Type 2 Macrophage

Macrophage Cell Counts and Population Fractions on Transitional ECM

Primary Tumor

Metastatic Tumor
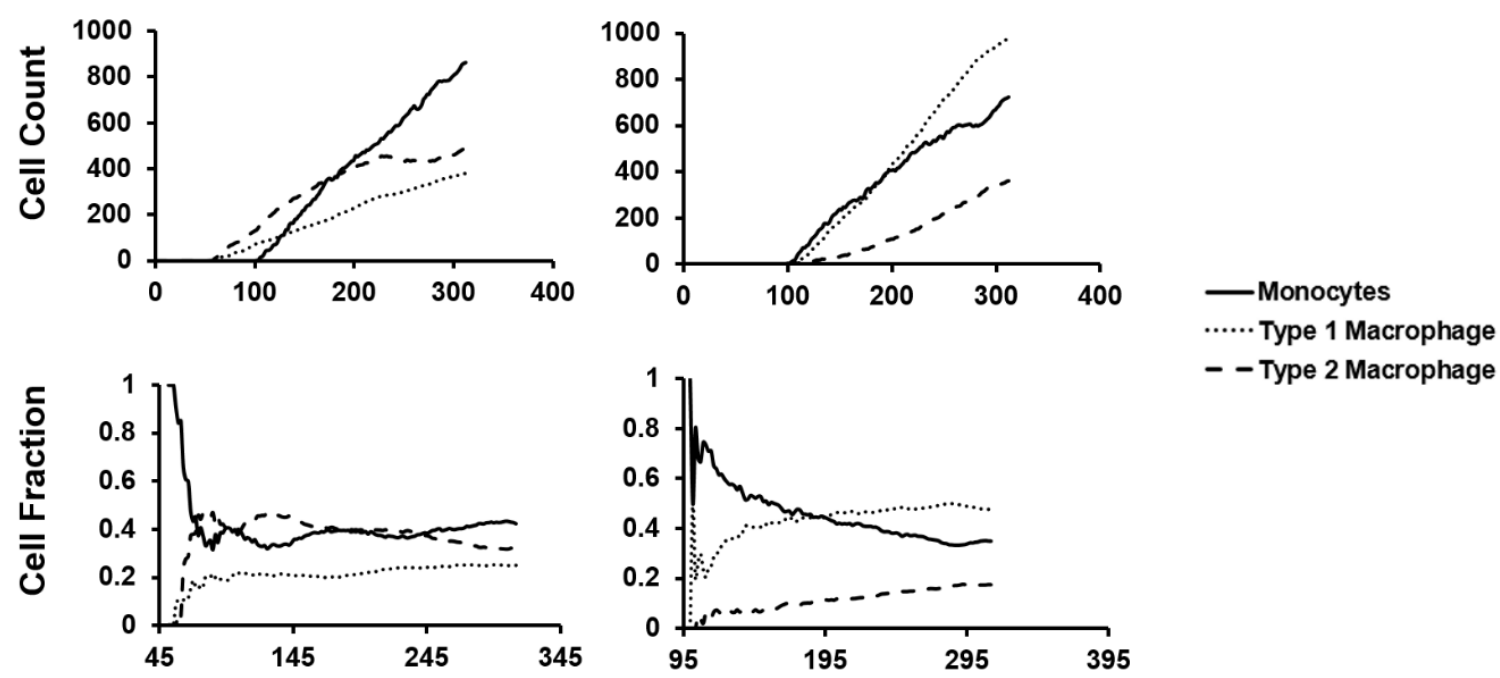

Time (h) 


\section{Discussion}

Macrophages are one of the key immune cell populations that are associated with tumor growth, invasion, and migration throughout all stages of cancer development ad progression [88, 227, 228]. While they may be initially recruited to the tumor site in the capacity of normal host defense, exerting tumoricidal activity, often tumors take this opportunity to exploit their proximity to these immune cells by sending signals to divert and induce them to cease action against the tumor. Macrophages then become obligate partners of circulating tumor cells, subverting the local environment to facilitate invasion, niche attachment, and neovascularization to promote metastatic growth and survival [229-231]. Although the functions that TAMs perform in the tumor microenvironment are unique compared to normal, non-tumor-associated macrophages, phenotypically they share the activation markers as M1 and M2 macrophages, and exhibit differential tumor effector expression profiles, both in a population location-dependent manner. Thus, having the ability to visualize their proximity to tumor stroma (classified by oxygenation threshold) and surrounding vasculature, is important with determining their functionality [232-234]. This model provides visualization of positional macrophage clustering, and also simulates tumor growth based on microenvironmental clues disseminated from hypoxic tumor regions, giving a clear picture of the tumor makeup with regard to oxygenation and general nutrient availability, and defining phenotypically the TAMs that share activation markers and tumor effector expression profiles with that of alternatively-activated M2 macrophages. 
It is well-known that trophic TAMs are propagated from either tissueenvironment monocytes local to the tumor that are polarized to an M2-like phenotype, or from classically activated M1 macrophages that were initially recruited to the site to exert tumoricidal effect on the tumor, that due to stress signaling from the tumor, are repolarized to $M 2[88,212,235]$. Therefore as a method of validating macrophage signaling in this model system, the indirect transwell co-culture experiment, a more simple in vitro system [236, 237], was executed using BMDMs either seeded in various polarization states: naïve MФ unpolarized, LPS-activated M1 polarized, or IL-4-activated M2 polarized. The murine tumor cell lines evaluated in co-culture were derived from either primary or metastatic tumor nodules; they were seeded into the top transwell chamber insert, on a cell-impermeable porous membrane such that only secreted cytokines were able to be exchanged with the macrophages below. These experiments provided the basis for estimation of macrophage parameters, as they were used to calibrate the levels of macrophage cytokines for growth simulations. The mathematical model visualizes the interactions described here, of the macrophage populations and tumor cells, by using data from ELISA assays to calibrate secreted cytokines from cell culture supernatants to cytokine levels diffused into cell [223] system matrix of the model [215].

In addition to having visualization of the cytokine diffusion gradients, the populations of polarized macrophages themselves are visible with respect to the field of established and nascent vasculature. This vascular grid, from which the monocytes extravasate prior to polarization to their activated states, is the matrix 
upon which macrophages are clustered, and in this way the phenotypes of the heterogeneous differential populations of macrophages can be depicted in overlay of the proliferating tumor cells. The tumor mass is visualized by regions of differential oxygenation, which here represents oxygen, glucose, and nutrients in general for simplification. With only the normoxic sections being capable of proliferation, modeled as the expansion of the tumor boundary through the matrix scaffold, the model can capture the stress signaling of hypoxic regions via release of angiogenic factors and cytokine signals. Vascular sprouts and extravasation of macrophages is triggered, correlating to VEGF macrophage recruitment. Cytokine signals act on monocytes and macrophages, modeling the capability the tumor has to subvert monocytes and macrophages into generating the nutrient access via neoangiogenesis and matrix access via invasion of degradation of connective tissues of the ECM. Normoxic tumor cells continue to grow so long as they have a sufficient oxygen supply.

In addition to incorporating macrophage cytokine parameters, the extracellular matrix density was also a part of the TME in the model. The interaction between the tumor cells and macrophages is explored in the context of seeding to the selective "soils" of the homeostatic control LECM vs LECM derived liver tissue that has undergone alcoholic injury [45, 67]. Migration assays on these two experimental ECM constructs showed that both tumor cell lines had optimal/higher migration across membrane to lower chamber on LECM derived from ethanol fed animals vs the control pair fed mice. This finding was recapitulated in the model using experimental data to modulate ECM thickness, 
correlating to the increase in fibrous density that would occur in the transitional remodeling of the ECM in response to alcohol exposure. The simulations were executed via calibrating the production and degradation constants of the ECM, resulting in differential pattern of ECM, correlating to the aforementioned alterations to ECM protein composition explored in Chapter II. The tECM simulation produced a larger metastatic tumor radius than on cECM, as measured by its final tumor radius.

With levels of M1 and M2 macrophages calibrated to those found in the co-culture assay, simulations produced growth results that illustrate the potent antitumoral effect of M1 macrophages in the system, as tumor growth in all cases was decreased compared to control simulations run with no differentiated macrophages. In general, metastatic tumors exhibited decreased growth compared to primary tumors, again likely owing to the high M1 cytokine (TNF- $\alpha$ ) levels measured in these cells. Interestingly, both primary tumor simulations had larger areas of necrosis than the metastatic tumor simulations, which had mostly hypoxic blue cores. The primary tumor cells tended toward a balance between M1 and M2 populations, while the metastatic tumors had a higher infiltration of M1 subtypes, which resulted in growth restriction relative to all other cases. Macrophages took longer time to appear in the metastatic cases in comparison to the primary cases, due to the vascular density for metastatic tumors (modeled after hepatic vasculature), and therefore a differential in oxygen thresholds [45, 223]. Because of this, the metastatic cases took longer to become hypoxic and 
start releasing TAF for recruitment of M2 growth-promoting factors [238]. There were nominal differences in tumor mass among the control simulation group.

The results here move forward the original work on this model (Mahlbacher et al. [215]), by experimentally validating the modeling of both primary and metastatic lesion growth, modulated by macrophage interactions. Positional and quantitative data gave insight on the nuances of macrophage infiltration of the tumor mass as well as how the distribution of subtype populations differs between primary and metastatic cell types. The rate of growth and final tumor mass simulated by the model correlated well with the in vivo data, both experiments indicating that the primary tumor mass grows larger with necrotic cores, while the metastatic nodes are typically smaller in size and more hypoxic, in agreement with the reported incidence of necrosis in primary tumor spheroids [239-243]. The organ site was also taken into account in the grid composition of this simulation, with the metastatic tumor cells having a denser grid than the primary tumor cells, reflecting the higher vascularization of liver relative to the lung [244]. In the resulting tumor masses, the growth restriction of the metastatic tumors has allowed for more effective oxygenation of the smaller tumor mass. Though not verified in vivo, the simulations reflected the increased M1 cytokine levels in the metastatic tumors, as calibrated from co-culture assays, which contributes to the antitumoral activity exhibited in the metastatic simulations [245].

Future directions for this work include expanding cytokine panel to include additional signaling molecules, thereby having a more complex milieu of 
cytokines from heterogeneous macrophage populations, giving the model output of tumor growth more translational value. Clinical implications could include therapies that are better targeted to the differential immune cell phenotypes and ECM composition states. Augmenting this model with data from further ECM experiments would help to more accurately calibrate the model to ECM changes that affect [tumor] cell attachment and subsequent immune activation. Data representing ECM adhesion characteristics could also be employed here, providing information on the proteomic adhesion protein profile. Parameters regarding both stimulatory and inhibitory effects of ECM protein would build complexity into the simulation of microenvironmental interactions, extending the utility of this application. The success of this model system in recapitulating the experimental conditions and outcomes as borne out in the data shows potential for a combined mathematical/experimental system to be applied to various other tumor-related paradigms. With suitable expansion and built in capacity, this will allow exploration of multiple other factors of the microenvironment that may influence the behavior of metastatic tumor growth under similar conditions. 
CHAPTER VI

\section{DISCUSSION AND CONCLUSIONS}

\section{A. Restatement of goals and questions}

The goal of the work outlined herein was to explore the hypothesis of whether alcohol-induced transitional ECM changes influence the potential of metastatic tumor seeding in the liver, and whether resulting immunomodulatory signals from macrophages in the TME facilitate tumor progression. This group had demonstrated that alcohol consumption, and resulting steatosis, has the ability to impact the composition of the extracellular matrix, creating the phenomena of the transitional ECM [45]. In this state, the expression of ECM proteins is altered in a way that can precipitate permanent alteration if the liver insult is sustained, but otherwise can be resolved if the insult is acute and minimally toxic. This was shown in Chapter III, where ECM composition under experimental liver injury was used to model altered integrin binding in computational kinetic simulations. Chapter IV characterizes experimental changes to macrophage polarization, to assess the conditions under which classically activated macrophages can be switched to alternatively activated macrophages, to correlate to changes in inflammatory microenvironment in hepatocellular carcinoma. Finally, Chapter $V$ describes the application of a spatio-temporal mathematical model to evaluate tumor growth under altered 
ECM conditions with repolarized macrophages. Taken together, these studies establish a new approach for employing computational modeling to investigate key features of the tumor microenvironment.

\section{B. Major findings of this dissertation}

1. Altered hepatic ECM protein composition resulting from ECM remodeling associated with liver injury changes binding profile for cognate integrin receptors

Previous studies from the Arteel group have demonstrated that the hepatic extracellular matrix may affect the early stages of alcoholic liver disease via transitional remodeling of the matrix [44, 45]. Using proteomic data analysis, it was determined that alcohol exposure activates a dynamic response of the murine hepatic ECM proteome. As several of the ECM proteins identified to be quantitatively or qualitatively changed by alcohol exposure are ligands for integrin receptor binding, and integrin signaling plays an important role in the downstream cellular functions supported by the ECM, it was important to explore how altered ECM affects integrin binding. The goals of Chapter III were to 1) develop a mathematical model to systematically explore changes in hepatic phenotype driven by aberrant ECM/integrin interactions, and 2) to simulate binding kinetics of ECM proteins to their integrin receptors to investigate whether binding is affected by altered ECM expression.

First, to establish a mathematical model, a two-step model described by Masson-Gandais et al. and Wanant et al. was modified to simulate sequential binding of a dimeric subunit, replicating the integrin $\alpha$ subunit binding prior to the 
$\beta$ subunit $[111,119]$. This two-step process reflected a divalent kinetics model with the $\alpha$ subunit existing as the high affinity site, and the $\beta$ subunit as the low affinity site. In addition to binding processivity of individual receptors, ligand binding to distinct integrins favors subsequent binding by other receptors (i.e. focal adhesion clustering). Finally, promiscuity among the repertoire of ECM ligands and integrin receptors, particularly RGD-binding ligands, implies a differential pattern of binding relative to the amounts of substrate available.

To explore these interactions as a system, modeling of integrin receptor binding kinetics, considering a divalent receptor and affinities for each ligand species, a single-species integrin profile was established. The data from proteomic analyses provided substrate concentrations used to calibrate model simulations. As expected, altered ECM composition affected binding kinetics of integrin receptors. Increased affinity and avidity for ligand was reflected in simulation curves, corresponding with increased ECM ligand as reflected in proteomic data. Both the binding curves for fully occupied divalent receptors and fully occupied aggregate receptor pairs exhibited the same affinity increase. Additionally, simulation data indicated that upregulated ECM proteins reached steady state values in shorter time, and that aggregation of receptors produced positive cooperativity.

This aim proposed a modeling framework that, although initially evaluated with data from a model of fulminant fibrosis, could be employed to explore other liver pathologies and how the transitional remodeling of the ECM affects ECMintegrin interactions. By testing homeostatic conditions against experimental 
treatment models, this platform could be broadly employed to predict or confirm changes in integrin binding (and by extension, signaling) caused by remodeling of the hepatic ECM in response to insult or injury.

\section{Take-home points}

- Alcohol exposure activates transitional remodeling of hepatic extracellular matrix

- Altered ECM protein expression within matrix affects cognate integrin binding kinetics

- Mathematical modeling of integrin binding kinetics shows increased affinity and avidity for ligand in experimental groups exhibiting upregulated ECM proteins

Text Box 6.1

2. Arsenic attenuates lipopolysaccharide-stimulated polarization in murine bone marrow derived macrophages

As described in Chapter 1 Section 6, macrophages are a key component of the tumor microenvironment throughout all stages of tumor growth and progression, including metastasis. An important determinant of their actions and the characteristics of the secreted effector molecules they release is their phenotypic polarization status. Whereas classically activated macrophages exhibit a proinflammatory response, alternatively activated macrophages are often more pliant to tumor cells and work in concert with them to facilitate attachment and invasion. As such, the goals of the experiments in Chapter IV were to 1) characterize both the $M 1$ and $M 2$, macrophage activation response in murine bone marrow derived macrophages, 2) test the effect of arsenic on these 
polarization responses, 3 ) identify potential miRNAs that may regulate these effects.

While chronic exposure to high concentrations of arsenic has been directly linked with a myriad of possible health risks, the concentrations of arsenic required to cause disease are generally considered higher than those found in the U.S. drinking water supply [152]. Subacute and chronic low-grade exposures are therefore important to research to assess its contribution to exposure biology in living organisms. Some studies indicate that moderate to low arsenic exposure may modify disease risk from other, separate etiologies. Previous studies by this group indicated that sub-toxic arsenic exposure enhanced recruitment of proinflammatory macrophages to the mouse liver during experimental NAFLD [155]. Indeed, here the a priori hypothesis was therefore that arsenic would enhance LPS-induced M1 phenotype, contributing to macrophage mediated inflammation.

The results herein indicate that low concentration of arsenite attenuated the LPS-mediated induction of CD11c expression, a marker of M1 polarization, in addition to the associated abrogation of classic proinflammatory markers in treated macrophage populations. In agreement with these findings, low dose arsenic exposure was reported to attenuate the innate immune response to influenza A (H1N1) viral infection in mice with a significant decrease in the total number of $\mathrm{CD} 11 \mathrm{C}^{+} / \mathrm{CD} 103^{+}$, and $\mathrm{CD} 11 \mathrm{C}^{+} / \mathrm{B} 220^{+}$dendritic cells [180]. This effect of arsenite is independent of cytotoxicity, as arsenite treatment at all concentrations, with the exception of $500 \mathrm{ppb}$, did not cause any significant 
reduction in BMDMs cellular viability. In contrast, arsenite did not affect the number of $\mathrm{CD} 206^{+} / \mathrm{F} 480^{+} / \mathrm{CD} 11 \mathrm{~b}^{+}$BMDMs or the intensity of CD206 in the presence/absence of IL-4. These results suggest that arsenite alone does not affect macrophage polarization, but has the potential to modify or deregulate the toll-line receptor 4 (TLR4)-mediated M1 response.

Microarray analysis of LPS treatment produced data similar to what has been previously described for miRNAs associated with TLR4 activation and inflammatory murine macrophages [198]. The current study is the first to report the effect of arsenite exposure on miRNA expression in BMDMs. The linear contrast comparing miRNA expression of LPS $+\mathrm{As}(\mathrm{III})$ cells vs. those treated with LPS alone revealed that miR-301b-3p as the most downregulated (Table 1), with a $\log 2 \mathrm{FC}$ of -3.33 and $p$ value of 0.0008 . This miR was chosen for follow up analysis and exploration. First, it was verified as being downregulated by comparing all treatment groups via qRT-PCR quantitation. IPA analysis indicated strong correlations with cancer for the miR-130a family, which may be related to the increased expression of TH2-like cytokines of tumor cells coopting tumor associated macrophages (TAMs), and the exploitation of their tissue trophic and repair functions by invading tumor cells [212]. This effect of arsenite could potentially be attributed to its effects on miRNA expression profiles of macrophage populations. Based on these findings, it is proposed that low dose arsenic exposures could attenuate the proinflammatory response of the innate immune system, which in this system may indicate potential impairment of the bactericidal macrophage activity in vivo. 
Text Box 6.2

Take-home points

- Classic M1 and M2 polarization was achieved with LPS and IL4 treatment, respectively, as verified via FACS analysis of $M \theta$ markers and mRNA expression of related cytokines validated macrophage polarization

- Arsenic alone did not significantly affect polarization of BMDMs; however In the presence of LPS, As(III) was able to attenuate LPS-induced M1 polarization

- Various miRNAs significantly associated were identified, though miRNA $301 \mathrm{~b}$ exhibited the highest fold change in response to LPS-As treatment compared to LPS treatment alone

3. Immunomodulatory stimuli and the density of extracellular matrix in the tumor microenvironment affects in silico tumor growth in a spatio-temporal mathematical model of tumor growth

Chronic hepatic inflammation promotes dramatic remodeling of the liver

ECM, which is an important factor in both fibrotic disease and cancer, contributing to tumor progression and metastasis [69, 246]. The long-term efficacy of cancer therapeutics is limited by metastatic disease, and often metastases exhibit selective tropism to the liver, particularly from primary lung and colorectal cancers. Although the mechanisms of metastatic organotropism are poorly understood, it is clear that communication between cancer cells and the target microenvironment are key. Indeed, several of the hallmarks of metastasis, such as proliferation, angiogenesis and apoptosis, are hypothesized to be mediated by altered integrin signaling $[17,18,144]$. Interestingly, alcohol consumption is a known risk factor for increasing metastasis to the liver, suggesting that early subclinical alcohol-mediated liver ECM remodeling may impact seeding and colonization of hepatic metastases $[47,50]$. We hypothesized that transitional alterations to the hepatic matrisome create a more 
favorable environment for dissemination of metastases to the liver, and modified the mathematical model developed by Mahlbacher et al. to simulate macrophage populations and ECM density changes and their effects on tumor growth [215].

Key findings indicated that the presence of $\mathrm{M} 1+\mathrm{M} 2$ heterogeneous macrophage population subtypes decreased tumor growth compared to controls where solely ECM density was modulated and only undifferentiated monocytes populated the grid. Overall, metastatic tumors exhibited decreased growth compared with primary tumors, on both $\mathrm{CECM}$ and tECM scaffolds. However, the tECM actually increased tumor growth in metastatic tumors, while decreasing growth of primary tumors. A potential theory for further exploration could be that the metastatic tumor cells, poised as they are for circulation, could be more effective at induction of classically activated macrophages, thereby instigating antitumoral activity at the onset of metastatic seeding. With successful neoangiogenesis, establishment of metastatic niche can progress and conversion of monocytes to tumor-associated macrophages could subvert the microenvironment to favor continued proliferation. Further investigation of this would require additional parameters to be set in model and increased timespan following tumor "seeding" to the matrix. 


\section{Take-home points}

- Primary and metastatic tumor cell lines exhibited increased migration on alcohol-exposed ECM growth substrate

- Metastatic tumor cell lines in indirect co-culture with naive BMDMs showed increased M1 cytokine markers in vitro; M2 cytokine markers were higher in tumor co-cultures than control BMDMs

- Computational model showed decreased tumor growth in presence of $M 1+M 2$ macrophages, and metastatic tumors exhibited decreased growth compared to primary tumors

- Transitional ECM scaffold decreased tumor growth in primary tumors, while increasing tumor growth for metastatic tumors

- Mathematical model applied herein can aid with investigating tumor growth under complex interactions of macrophage cytokine signaling and varying ECM density

\section{Significance of new findings}

As discussed in Chapter I, liver ECM is responsive to microenvironment, integrating cell signals to coordinate diverse pathways of action; its interaction with parenchymal function is critical in maintaining tissue integrity and nonpathogenic matrix remodeling for tissue repair [7]. Dyshomeostasis of matrix turnover is a key feature of almost every form of chronic liver disease, regardless of etiology; e.g., conditions that result from either direct or secondary liver insult, whether from alcohol exposure, metabolic disease, or exogenous toxicants.

Thereby, the ECM can be altered via inflammatory cascades that trigger progressively irreversible remodeling of the ECM. One of the primary aims of this dissertation was to utilize proteome data analysis of the hepatic ECM to explore 
integrin-ECM interactions. The experiments in Chapter III establish a divalent, aggregated integrin receptor model that simulated the binding kinetics of integrin receptors in response to experimental upregulation of ECM proteins, as exhibited in liver injury in response to $\mathrm{CCl}_{4}$ treatment. The results from this study suggest that integrin receptor affinity and ligand avidity increases in response to increased availability of ECM proteins from remodeled hepatic ECM. This model could be utilized for evaluating other remodeling events caused by separate liver pathologies.

Macrophages are key players in tumor growth and progression, and their downstream affects depend largely on their polarization status. Often tumor cells using cytokine signaling to effectively switch the polarization of macrophages to support a more trophic phenotype that allows for ECM invasion and migration of tumor cells. Novel findings in Chapter IV show that arsenic has an attenuating effect on M1 proinflammatory polarization of bone marrow derived murine macrophages. This effect of arsenic could potentially be attributed to its effects on miRNA expression profiles of macrophage populations. In particular, miR$301 \mathrm{~b}$ was shown to have the greatest expression change in response to treatment with LPS+Arsenic vs LPS alone. It is very possible that modulation of miRNAs, as shown in this study, controls the action of activin A in polarization switching; in this case, arsenic exposure upregulated expression of activin A, and subsequently the M1 polarization of treated macrophages was altered.

Finally, the third aim of this dissertation sought to develop a modeling approach that could be used to assess tumor growth while modifying the 
macrophages and ECM of the tumor microenvironment. Cancer progression, invasion, and metastatic spread is facilitated by the ECM, while macrophages contribute microenvironmental cues that alter the ability of tumor cells to attach, invade the ECM, and migrate. In the model system applied in Chapter V, the roles of both M1 and M2 macrophages are visualized as cell populations, and the density of ECM is modified to recapitulate hepatic ECM remodeling in response to alcohol exposure. In each module, data from in vitro experiments are used to calibrate macrophage cytokine levels and ECM density, to provide translational value to the computational model. The simulations showed that tumor cell growth was enhanced when ECM turnover was held at levels that sustained matrix production, while preventing overproduction. M2 cytokine signaling was important for release of tumor nutrients and oxygenation to reach hypoxic regions of growing tumor mass. This model system could be utilized to simulate tumorinduced switching of macrophage polarization, in conjunction with assimilation of ECM proteome data to configure ECM density, to test which microenvironmental conditions optimize tumor growth in silico.

\section{Strengths and weaknesses}

\section{Strengths}

There are several strengths of the work presented in this dissertation.

First, this work uses novel mathematical modeling approaches to study various biological questions that act in synergy to affect tumor growth and dissemination. A kinetic binding model modified to simulate integrin binding to ECM proteins 
provides a novel way to employ proteome data to simulate dynamic changes to the hepatic extracellular matrix.

Additionally, this dissertation explored macrophage polarization and treatments that can attenuate the classically activated state of M1 macrophages. As tumors can secrete effectors that affect macrophage polarization in the tumor microenvironment, understanding how these switches are made is important. This work identified several miRNAs that could potentially modulate the macrophage response, and selected one lead miRNA that can be followed in further studies to determine the exact mechanisms of arsenic's ability to attenuate the M1 macrophage response when treated with LPS in vitro.

A second spatio-temporal model was modified to simulate tumor growth in response to macrophage cytokine signaling and its effects on circulating monocytes and neoangiogenesis for tumor vascularization. This model can simulate multiple aspects of the tumor microenvironment and visualize their interaction in terms of cell populations and diffused chemoattractants. Importantly, these models were both calibrated with biological endpoints and validated with in vitro experiments.

\section{Weaknesses}

Although the computational models used in this work are a key strength, there are some drawbacks in terms of model complexity. Since integrin binding in real time should be modeled stochastically, the dimeric aggregate model developed here should be integrated into a stochastic system to allow for 
prediction of binding species in a probabilistic manner. Additionally, there is no translational component to this first aim, and the mathematical model has not been tested in a system of alcohol exposure as yet.

In addition to this, though miRNAs were identified as lead candidates for influencing macrophage polarization in response to LPS+Arsenic, subsequent studies using genetic modification to either knock-out or overexpress these miRNAs would need to be done to establish whether there is a true effect of these miRNAs on macrophage polarization phenotypes in bone marrow derived macrophages.

The spatio-temporal model utilized was calibrated using data from in vitro transwell assays of tumor migration and tumor-macrophage co-cultures. Further information could be provided in the model's proteomic module if data from ECM proteome, or adhesome, could be incorporated into the model. Additionally, there was no successful in vivo model that was generated to further validate the transwell results, that alcohol-exposed livers are more permissive to tumor metastases. This would have provided additional translational value to the in silico simulation.

Summary Table 6.1

\begin{tabular}{|c|c|}
\hline \begin{tabular}{|l} 
Strengths \\
\end{tabular} & Limitations \\
\hline $\begin{array}{l}\text { - Novel integrin binding model of } \\
\text { divalent aggregated receptors } \\
\text { showing increased affinity and } \\
\text { avidity for ECM protein ligands }\end{array}$ & $\begin{array}{l}\text { - Prediction of binding species } \\
\text { remain uninvestigated due to lack } \\
\text { of probabilistic simulations }\end{array}$ \\
\hline $\begin{array}{l}\text { - Novel finding of specific miRNAs } \\
\text { that may play a role in the } \\
\text { attenuation of the M1 classical } \\
\text { macrophage activation state in }\end{array}$ & $\begin{array}{l}\text { - Lack of follow up studies to verify } \\
\text { mechanisms by which identified } \\
\text { miRNAs affect macrophage } \\
\text { polarization }\end{array}$ \\
\hline
\end{tabular}


response to LPS+Arsenic treatment in BMDMs

- Validation of mathematical tumor model, using in vitro data on tumor cell microenvironments relating to macrophage cytokine signaling and hepatic ECM density
- Lack of translational murine model of enhanced liver metastasis in a model of alcohol exposure; requires further investigation

\section{E. Future Directions}

While the experiments described in this dissertation addressed computational approaches that could improve tumor-microenvironment modeling and simulation, it has also created new questions to be followed in future studies. A few of these are addressed below.

1. Could a stochastic model of integrin binding predict binding species?

The experiments described in Chapter III utilize mathematical modeling to systematically explore changes in hepatic phenotype driven by altered ECMintegrin interactions. While a simple integrin clustering scheme was developed to determine binding affinity of the various ECM proteins to their various cognate integrin receptors, this model could be built into a more complex model that could predict integrin binding species using probabilistic methods. In this way, proteomic data from alcohol-exposed hepatic ECM could be used to simulate binding by using a model of concurrent binding of multiple receptor and ligand 
species, to develop an integrated binding phenotype based on the quantitative hepatic ECM proteomic signatures from alcohol-exposed livers.

Summary Table 6.2

\begin{tabular}{|l|l|}
\hline \multicolumn{2}{|c|}{ Probabilistic Integrin Binding Model? } \\
\hline Rationale & $\begin{array}{l}\text { Prediction of integrin binding "profile" under various } \\
\text { conditions involving pathological remodeled hepatic ECM. }\end{array}$ \\
\hline Approach & $\begin{array}{l}\text { Use mathematical models to describe concurrent binding } \\
\text { of divalent receptor aggregates using stochastic } \\
\text { simulations. }\end{array}$ \\
\hline Expected Results & $\begin{array}{l}\text { ECM protein expression induced by liver remodeling in } \\
\text { response to alcohol exposure will significantly alter } \\
\text { integrin binding profile. }\end{array}$ \\
\hline
\end{tabular}

2. What is the mechanism by which miR-301b modulates macrophage polarization attenuation in LPS+Arsenic-treated BMDMs?

In the second aim, macrophage polarization was explored in terms of using microarray expression analysis to identify miRNAs in BMDMs that had significant expression changes in response to treatment with LPS+Arsenic. Of those, miR-301b was identified as a lead candidate in miRNAs that played a role in modulating macrophage activation. While the results did validate this altered expression, additional studies would need to be undertaken in order to determine the mechanism by which miR-301b works in this system. 
Summary Table 6.3

Role of miR-301b in attenuating M1 macrophage response?

\begin{tabular}{|l|l|}
\hline Rationale & $\begin{array}{l}\text { Elucidate the specific pathway by which the arsenic- } \\
\text { induced downregulation of miR-301b acts to attenuate } \\
\text { that classic inflammatory macrophage response in vitro. }\end{array}$ \\
\hline Approach & $\begin{array}{l}\text { Transfect BMDMs to over-express miR301b to verify } \\
\text { expression changes from microarray results. Then use } \\
\text { these cells in downstream experiments to identify } \\
\text { mechanisms. }\end{array}$ \\
\hline Expected Results & $\begin{array}{l}\text { Activin A action, which may be controlled by modulation } \\
\text { of specific miRNAs, may be the key mediator in } \\
\text { polarization switching in this system. }\end{array}$ \\
\hline
\end{tabular}

3. Can translational value of computational tumor growth model be enhanced by in vivo model data?

The experiments in Chapter $V$ provide calibration data for the tumor growth model. In particular, transwell migration assay data that showed increased migration of primary and metastatic tumor cell lines on alcoholexposed murine liver ECM growth substrate, were used to calibrate ECM density and turnover. Indirect co-culture transwell assays of macrophage and tumor cells resulted in data that showed that metastatic tumor cells were able to attenuate M1 macrophages, and this was modeled in macrophage populations and their associated chemoattractants in the in silico model. It is possible that further validation from in vivo model data could make the in silico model more robust and translational. This could be achieved by successful establishment of a model of liver metastasis in alcohol-exposed mice, and using the clinical/pathological study endpoints to calibrate the in silico model. 


\begin{tabular}{|l|l|}
\hline \multicolumn{2}{|c|}{ Improvement of translational value of in silico tumor model? } \\
\hline Rationale & $\begin{array}{l}\text { Alcohol-induced transitional remodeling of the hepatic } \\
\text { ECM enhances metastatic seeding via macrophage- } \\
\text { mediated tumor-ECM interactions. }\end{array}$ \\
\hline Approach & $\begin{array}{l}\text { Establish an in vivo model of cancer metastasis to the } \\
\text { liver, and use the pathological study endpoint data to } \\
\text { calibrate in silico model. }\end{array}$ \\
\hline Expected Results & $\begin{array}{l}\text { Translational value of model will be improved by addition } \\
\text { additional modules to simulate from in vivo data. }\end{array}$ \\
\hline
\end{tabular}

\section{F. Summary and conclusions}

The overall goal of the dissertation described herein addresses the hypothesis that transitional alterations to the hepatic matrisome create a more favorable environment for dissemination of metastases to the liver, and that specific integrins mediate these modifications. Although several studies have investigated the role of the ECM in metastasis, the concept of leveraging the experimentally determined composition of the liver ECM under various conditions to inform a kinetic integrin binding model is novel and has implications for establishing a profile of integrin-ECM interaction. This work contributes to the understanding of the role of the hepatic ECM microenvironment in influencing the expression of integrin receptors on invading circulatory tumor cells.

This work in Chapter III establishes a novel approach, in that we are employing top-down systems biology data with the use of proteomics, along with a bottom-up mathematical modeling strategy to study the dynamics and kinetics of system component interactions. The work explores new hypotheses of the role 
of the matrisome in metastatic seeding. In addition, the experiments in Chapter IV begin to explore the attenuation of M1 polarization of macrophages, which is a phenomenon that often occurs in the tumor microenvironment when tumor cells induce polarization switching in circulating macrophages to facilitate their invasion and proliferation.

The systems biology approach in Chapter $\mathrm{V}$ incorporates spatio-temporal tumor growth simulation, and translational validation of in silico results via relevant murine tumor cell culture models. Specifically, macrophage populations are modeled to investigate their polarization status and its effects on tumor growth and neovascularization. Additionally, the hepatic ECM is modeled in density and turnover of the matrix in the in silico system, to give a marginally complex depiction of interactions of at least three separate elements of the tumor microenvironment. Taken together, the data presented here developed a new approach to examine complex interactions of tumor-ECM interactions and the interplay with the innate immune system. 


\section{REFERENCES}

1. Rozario T, DeSimone DW. The extracellular matrix in development and morphogenesis: a dynamic view. Developmental biology. 2010;341(1):126-40. Epub 2009/10/27. doi: 10.1016/j.ydbio.2009.10.026. PubMed PMID: 19854168; PubMed Central PMCID: PMCPMC2854274.

2. Mao JR, Bristow J. The Ehlers-Danlos syndrome: on beyond collagens. The Journal of clinical investigation. 2001;107(9):1063-9. Epub 2001/05/09. doi: 10.1172/jci12881. PubMed PMID: 11342567; PubMed Central PMCID: PMCPMC209288.

3. Coulombe PA, Kerns ML, Fuchs E. Epidermolysis bullosa simplex: a paradigm for disorders of tissue fragility. The Journal of clinical investigation. 2009;119(7):1784-93. Epub 2009/07/10. doi: 10.1172/jci38177. PubMed PMID: 19587453; PubMed Central PMCID: PMCPMC2701872.

4. Sewry CA, Muntoni F. Inherited disorders of the extracellular matrix. Current opinion in neurology. 1999;12(5):519-26. Epub 1999/12/11. PubMed PMID: 10590888.

5. Bonnans C, Chou J, Werb Z. Remodelling the extracellular matrix in development and disease. Nature reviews Molecular cell biology. 2014;15(12):786-801. Epub 2014/11/22. doi: 10.1038/nrm3904. PubMed PMID: 25415508; PubMed Central PMCID: PMCPMC4316204.

6. Guo XD, Johnson JJ, Kramer JM. Embryonic lethality caused by mutations in basement membrane collagen of C. elegans. Nature. 1991;349(6311):707-9. Epub 1991/02/21. doi: 10.1038/349707a0. PubMed PMID: 1996137.

7. Cox TR, Erler JT. Remodeling and homeostasis of the extracellular matrix: implications for fibrotic diseases and cancer. Disease models \& mechanisms. 2011;4(2):165-78. Epub 2011/02/18. doi: 10.1242/dmm.004077. PubMed PMID: 21324931; PubMed Central PMCID: PMCPMC3046088.

8. Lu P, Takai K, Weaver VM, Werb Z. Extracellular matrix degradation and remodeling in development and disease. Cold Spring Harbor perspectives in biology. 2011;3(12). Epub 2011/09/16. doi: 10.1101/cshperspect.a005058. PubMed PMID: 21917992; PubMed Central PMCID: PMCPMC3225943.

9. Lu P, Weaver VM, Werb Z. The extracellular matrix: A dynamic niche in cancer progression. 2012;196(4):395-406. doi: 10.1083/jcb.201102147 \%J The Journal of Cell Biology.

10. Naba A, Clauser, K.R., Ding, H., Whittaker, C.A., Carr, S.A., Hynes, R.O. The extracellular matrix: Tools and insights for the "omics" eral. Matrix Biol. 2016;49:10. doi:

10.1016/j.matbio.2015.06.003.

11. Hynes RO, Naba A. Overview of the matrisome--an inventory of extracellular matrix constituents and functions. Cold Spring Harbor perspectives in biology. 2012;4(1):a004903. Epub 2011/09/23. doi: 10.1101/cshperspect.a004903. PubMed PMID: 21937732; PubMed Central PMCID: PMCPMC3249625.

12. Iredale JP, Thompson A, Henderson NC. Extracellular matrix degradation in liver fibrosis: Biochemistry and regulation. Biochimica et Biophysica Acta (BBA) - Molecular Basis of Disease. 2013;1832(7):876-83. doi: https://doi.org/10.1016/j.bbadis.2012.11.002.

13. Michalopoulos GK. Liver regeneration. Journal of cellular physiology. 2007;213(2):286300. Epub 2007/06/15. doi: 10.1002/jcp.21172. PubMed PMID: 17559071; PubMed Central PMCID: PMCPMC2701258. 
14. Campbell ID, Humphries MJ. Integrin structure, activation, and interactions. Cold Spring Harbor perspectives in biology. 2011;3(3). Epub 2011/03/23. doi: 10.1101/cshperspect.a004994. PubMed PMID: 21421922; PubMed Central PMCID: PMCPMC3039929.

15. Harburger DS, Calderwood DA. Integrin signalling at a glance. Journal of cell science. 2009;122(Pt 2):159-63. Epub 2009/01/02. doi: 10.1242/jcs.018093. PubMed PMID: 19118207; PubMed Central PMCID: PMCPMC2714413.

16. Humphries JD, Byron A, Humphries MJ. Integrin ligands at a glance. Journal of cell science. 2006;119(Pt 19):3901-3. Epub 2006/09/22. doi: 10.1242/jcs.03098. PubMed PMID: 16988024; PubMed Central PMCID: PMCPMC3380273.

17. Ganguly KK, Pal S, Moulik S, Chatterjee A. Integrins and metastasis. Cell adhesion \& migration. 2013;7(3):251-61. Epub 2013/04/09. doi: 10.4161/cam.23840. PubMed PMID: 23563505; PubMed Central PMCID: PMCPMC3711990.

18. Desgrosellier JS, Cheresh DA. Integrins in cancer: biological implications and therapeutic opportunities. Nature reviews Cancer. 2010;10(1):9-22. Epub 2009/12/24. doi: 10.1038/nrc2748. PubMed PMID: 20029421; PubMed Central PMCID: PMCPMC4383089. 19. Gulubova MV, Vlaykova TI. Significance of tenascin-C, fibronectin, laminin, collagen IV, alpha5beta1 and alpha9beta1 integrins and fibrotic capsule formation around liver metastases originating from cancers of the digestive tract. Neoplasma. 2006;53(5):372-83. Epub 2006/10/03. PubMed PMID: 17013531.

20. Whatcott CJ, Diep CH, Jiang P, Watanabe A, LoBello J, Sima C, et al. Desmoplasia in Primary Tumors and Metastatic Lesions of Pancreatic Cancer. Clinical cancer research : an official journal of the American Association for Cancer Research. 2015;21(15):3561-8. Epub 2015/02/20. doi: 10.1158/1078-0432.Ccr-14-1051. PubMed PMID: 25695692; PubMed Central PMCID: PMCPMC4526394.

21. Pandol S, Edderkaoui M, Gukovsky I, Lugea A, Gukovskaya A. Desmoplasia of pancreatic ductal adenocarcinoma. Clinical gastroenterology and hepatology : the official clinical practice journal of the American Gastroenterological Association. 2009;7(11 Suppl):S44-7. Epub 2009/12/05. doi: 10.1016/j.cgh.2009.07.039. PubMed PMID: 19896098; PubMed Central PMCID: PMCPMC4573641.

22. Oskarsson T. Extracellular matrix components in breast cancer progression and metastasis. Breast (Edinburgh, Scotland). 2013;22 Suppl 2:S66-72. Epub 2013/10/01. doi: 10.1016/j.breast.2013.07.012. PubMed PMID: 24074795.

23. Zhu J, Xiong G, Trinkle C, Xu R. Integrated extracellular matrix signaling in mammary gland development and breast cancer progression. Histology and histopathology. 2014;29(9):1083-92. Epub 2014/04/01. doi: 10.14670/hh-29.1083. PubMed PMID: 24682974; PubMed Central PMCID: PMCPMC4388417.

24. Todd JR, Ryall KA, Vyse S, Wong JP, Natrajan RC, Yuan Y, et al. Systematic analysis of tumour cell-extracellular matrix adhesion identifies independent prognostic factors in breast cancer. Oncotarget. 2016;7(39):62939-53. Epub 2016/08/25. doi: 10.18632/oncotarget.11307. PubMed PMID: 27556857; PubMed Central PMCID: PMCPMC5325338.

25. Hanker AB, Estrada MV, Bianchini G, Moore PD, Zhao J, Cheng F, et al. Extracellular Matrix/Integrin Signaling Promotes Resistance to Combined Inhibition of HER2 and PI3K in HER2(+) Breast Cancer. Cancer research. 2017;77(12):3280-92. Epub 2017/04/12. doi: 10.1158/0008-5472.Can-16-2808. PubMed PMID: 28396358; PubMed Central PMCID: PMCPMC5482178.

26. Seguin L, Desgrosellier JS, Weis SM, Cheresh DA. Integrins and cancer: regulators of cancer stemness, metastasis, and drug resistance. Trends in cell biology. 2015;25(4):234-40. 
Epub 2015/01/13. doi: 10.1016/j.tcb.2014.12.006. PubMed PMID: 25572304; PubMed Central PMCID: PMCPMC4380531.

27. Weber MR, Zuka M, Lorger M, Tschan M, Torbett BE, Zijlstra A, et al. Activated tumor cell integrin alphavbeta3 cooperates with platelets to promote extravasation and metastasis from the blood stream. Thrombosis research. 2016;140 Suppl 1:S27-36. Epub 2016/04/14. doi: 10.1016/s0049-3848(16)30095-0. PubMed PMID: 27067975; PubMed Central PMCID: PMCPMC4888909.

28. Yoshimura K, Meckel KF, Laird LS, Chia CY, Park JJ, Olino KL, et al. Integrin alpha2 mediates selective metastasis to the liver. Cancer research. 2009;69(18):7320-8. Epub 2009/09/10. doi: 10.1158/0008-5472.Can-09-0315. PubMed PMID: 19738067; PubMed Central PMCID: PMCPMC4857201.

29. Pickup MW, Mouw JK, Weaver VM. The extracellular matrix modulates the hallmarks of cancer. EMBO reports. 2014;15(12):1243-53. Epub 2014/11/10. doi: 10.15252/embr.201439246. PubMed PMID: 25381661; PubMed Central PMCID: PMCPMC4264927.

30. Blehm BH, Jiang N, Kotobuki $\mathrm{Y}$, Tanner K. Deconstructing the role of the ECM microenvironment on drug efficacy targeting MAPK signaling in a pre-clinical platform for cutaneous melanoma. Biomaterials. 2015;56:129-39. Epub 2015/05/03. doi: 10.1016/j.biomaterials.2015.03.041. PubMed PMID: 25934286; PubMed Central PMCID: PMCPMC4417483.

31. Mueller L, Goumas FA, Affeldt M, Sandtner S, Gehling UM, Brilloff S, et al. Stromal fibroblasts in colorectal liver metastases originate from resident fibroblasts and generate an inflammatory microenvironment. The American journal of pathology. 2007;171(5):1608-18. Epub 2007/10/06. doi: 10.2353/ajpath.2007.060661. PubMed PMID: 17916596; PubMed Central PMCID: PMCPMC2043521.

32. Poznyak V, Fleischmann A, Rekve D, Rylett M, Rehm J, Gmel G. The world health organization's global monitoring system on alcohol and health. Alcohol research : current reviews. 2013;35(2):244-9. Epub 2013/01/01. PubMed PMID: 24881333; PubMed Central PMCID: PMCPMC3908716.

33. Poznyak V, Rekve, D. WHO Global status report on alcohol and health 2018. 2018 Contract No.: ISBN: 978-92-4-156563-9.

34. Mokdad AH, Marks JS, Stroup DF, Gerberding JL. Actual causes of death in the United States, 2000. Jama. 2004;291(10):1238-45. Epub 2004/03/11. doi: 10.1001/jama.291.10.1238. PubMed PMID: 15010446.

35. (NIAAA) NIoAAaA. Alcohol-related traffic deaths 2010.

36. Rehm J, Mathers C, Popova S, Thavorncharoensap M, Teerawattananon Y, Patra J. Global burden of disease and injury and economic cost attributable to alcohol use and alcoholuse disorders. The Lancet. 2009;373(9682):2223-33. doi: https://doi.org/10.1016/S01406736(09)60746-7.

37. Moos RH, Moos BS. Rates and predictors of relapse after natural and treated remission from alcohol use disorders. Addiction (Abingdon, England). 2006;101(2):212-22. Epub 2006/02/01. doi: 10.1111/j.1360-0443.2006.01310.x. PubMed PMID: 16445550; PubMed Central PMCID: PMCPMC1976118.

38. Sanyal AJ, Yoon SK, Lencioni R. The etiology of hepatocellular carcinoma and consequences for treatment. The oncologist. 2010;15 Suppl 4:14-22. Epub 2010/12/09. doi: 10.1634/theoncologist.2010-S4-14. PubMed PMID: 21115577.

39. Pinter M, Trauner M, Peck-Radosavljevic M, Sieghart W. Cancer and liver cirrhosis: implications on prognosis and management. ESMO Open. 2016;1(2). 
40. Friedman SL. Molecular regulation of hepatic fibrosis, an integrated cellular response to tissue injury. The Journal of biological chemistry. 2000;275(4):2247-50. Epub 2000/01/25. PubMed PMID: 10644669.

41. Kisseleva T. The origin of fibrogenic myofibroblasts in fibrotic liver. Hepatology (Baltimore, Md). 2017;65(3):1039-43. Epub 2016/11/20. doi: 10.1002/hep.28948. PubMed PMID: 27859502; PubMed Central PMCID: PMCPMC5476301.

42. Pellicoro A, Ramachandran P, Iredale JP. Reversibility of liver fibrosis. Fibrogenesis \& tissue repair. 2012;5(Suppl 1):S26. Epub 2012/12/25. doi: 10.1186/1755-1536-5-s1-s26. PubMed PMID: 23259590; PubMed Central PMCID: PMCPMC3368758.

43. Massey VL, Beier JI, Ritzenthaler JD, Roman J, Arteel GE. Potential Role of the Gut/Liver/Lung Axis in Alcohol-Induced Tissue Pathology. Biomolecules. 2015;5(4):2477-503. Epub 2015/10/06. doi: 10.3390/biom5042477. PubMed PMID: 26437442; PubMed Central PMCID: PMCPMC4693244.

44. Poole LG, Arteel GE. Transitional Remodeling of the Hepatic Extracellular Matrix in Alcohol-Induced Liver Injury. BioMed research international. 2016;2016:3162670. Epub 2016/11/16. doi: 10.1155/2016/3162670. PubMed PMID: 27843941; PubMed Central PMCID: PMCPMC5098054.

45. Massey VL, Dolin CE, Poole LG, Hudson SV, Siow DL, Brock GN, et al. The hepatic "matrisome" responds dynamically to injury: Characterization of transitional changes to the extracellular matrix in mice. Hepatology (Baltimore, Md). 2017;65(3):969-82. Epub 2016/12/31. doi: 10.1002/hep.28918. PubMed PMID: 28035785; PubMed Central PMCID: PMCPMC5319876. 46. Hynes RO. Integrins: Bidirectional, Allosteric Signaling Machines. Cell. 2002;110(6):67387. doi: https://doi.org/10.1016/S0092-8674(02)00971-6.

47. Pöschl G, Seitz HK. ALCOHOL AND CANCER. Alcohol and Alcoholism. 2004;39(3):155-65. doi: 10.1093/alcalc/agh057.

48. Ratna A, Mandrekar P. Alcohol and Cancer: Mechanisms and Therapies. 2017;7(3):61. PubMed PMID: doi:10.3390/biom7030061.

49. Landskron G, De la Fuente M, Thuwajit P, Thuwajit C, Hermoso MA. Chronic inflammation and cytokines in the tumor microenvironment. Journal of immunology research. 2014;2014:149185. Epub 2014/06/06. doi: 10.1155/2014/149185. PubMed PMID: 24901008; PubMed Central PMCID: PMCPMC4036716.

50. Meadows GG, Zhang H. Effects of Alcohol on Tumor Growth, Metastasis, Immune Response, and Host Survival. Alcohol research : current reviews. 2015;37(2):311-22. Epub 2015/12/24. PubMed PMID: 26695753; PubMed Central PMCID: PMCPMC4590626.

51. Rybinski B, Franco-Barraza J, Cukierman E. The wound healing, chronic fibrosis, and cancer progression triad. 2014;46(7):223-44. doi: 10.1152/physiolgenomics.00158.2013. PubMed PMID: 24520152.

52. Ambade A, Satishchandran A, Szabo G. Alcoholic hepatitis accelerates early hepatobiliary cancer by increasing stemness and miR-122-mediated HIF-1 $\alpha$ activation. Scientific Reports. 2016;6:21340. doi: 10.1038/srep21340

https://www.nature.com/articles/srep21340\#supplementary-information.

53. Osna NA, Donohue TM, Jr., Kharbanda KK. Alcoholic Liver Disease: Pathogenesis and Current Management. Alcohol research : current reviews. 2017;38(2):147-61. Epub 2017/10/11. PubMed PMID: 28988570; PubMed Central PMCID: PMCPMC5513682.

54. Sirica AE, Gores GJ. Desmoplastic stroma and cholangiocarcinoma: clinical implications and therapeutic targeting. Hepatology (Baltimore, Md). 2014;59(6):2397-402. Epub 2013/10/15. doi: 10.1002/hep.26762. PubMed PMID: 24123296; PubMed Central PMCID: PMCPMC3975806. 
55. Magistri P, Leonard SY, Tang CM, Chan JC, Lee TE, Sicklick JK. The glypican 3 hepatocellular carcinoma marker regulates human hepatic stellate cells via Hedgehog signaling. The Journal of surgical research. 2014;187(2):377-85. Epub 2014/01/21. doi: 10.1016/j.jss.2013.12.010. PubMed PMID: 24439425.

56. Kunzmann AT, Coleman HG, Huang WY, Berndt SI. The association of lifetime alcohol use with mortality and cancer risk in older adults: A cohort study. PLoS medicine. 2018;15(6):e1002585. Epub 2018/06/20. doi: 10.1371/journal.pmed.1002585. PubMed PMID: 29920516; PubMed Central PMCID: PMCPMC6007830.

57. Bagnardi V, Rota M, Botteri E, Tramacere I, Islami F, Fedirko V, et al. Alcohol consumption and site-specific cancer risk: a comprehensive dose-response meta-analysis. British Journal Of Cancer. 2014;112:580. doi: 10.1038/bjc.2014.579

https://www.nature.com/articles/bjc2014579\#supplementary-information.

58. Bagnardi V, Rota M, Botteri E, Tramacere I, Islami F, Fedirko V, et al. Light alcohol drinking and cancer: a meta-analysis. Annals of Oncology. 2013;24(2):301-8. doi:

10.1093/annonc/mds337.

59. Rehm J. Light or moderate drinking is linked to alcohol related cancers, including breast cancer. BMJ : British Medical Journal. 2015;351.

60. Maeda M, Nagawa H, Maeda T, Koike H, Kasai H. Alcohol consumption enhances liver metastasis in colorectal carcinoma patients. Cancer. 1998;83(8):1483-8. Epub 1998/10/22. PubMed PMID: 9781941.

61. de Ridder J, de Wilt JH, Simmer F, Overbeek L, Lemmens V, Nagtegaal I. Incidence and origin of histologically confirmed liver metastases: an explorative case-study of 23,154 patients. Oncotarget. 2016;7(34):55368-76. Epub 2016/07/16. doi: 10.18632/oncotarget.10552. PubMed PMID: 27421135; PubMed Central PMCID: PMCPMC5342423.

62. Disibio G, French SW. Metastatic patterns of cancers: results from a large autopsy study. Archives of pathology \& laboratory medicine. 2008;132(6):931-9. Epub 2008/06/04. doi: 10.1043/1543-2165(2008)132[931:Mpocrf]2.0.Co;2. PubMed PMID: 18517275.

63. Adam R, de Gramont A, Figueras J, Kokudo N, Kunstlinger F, Loyer E, et al. Managing synchronous liver metastases from colorectal cancer: A multidisciplinary international consensus. Cancer Treatment Reviews. 2015;41(9):729-41. doi: https://doi.org/10.1016/j.ctrv.2015.06.006.

64. Valderrama-Trevino Al, Barrera-Mera B, Ceballos-Villalva JC, Montalvo-Jave EE. Hepatic Metastasis from Colorectal Cancer. Euroasian journal of hepato-gastroenterology. 2017;7(2):166-75. Epub 2017/12/05. doi: 10.5005/jp-journals-10018-1241. PubMed PMID: 29201802; PubMed Central PMCID: PMCPMC5670263.

65. Zhao H, Chen D, Cao R, Wang S, Yu D, Liu Y, et al. Alcohol consumption promotes colorectal carcinoma metastasis via a CCL5-induced and AMPK-pathway-mediated activation of autophagy. Scientific Reports. 2018;8(1):8640. doi: 10.1038/s41598-018-26856-w.

66. Williams RR, Horm JW. Association of Cancer Sites With Tobacco and Alcohol Consumption and Socioeconomic Status of Patients: Interview Study From the Third National Cancer Survey. JNCl: Journal of the National Cancer Institute. 1977;58(3):525-47. doi: 10.1093/jnci/58.3.525.

67. Kaplan RN, Rafii S, Lyden D. Preparing the "soil": the premetastatic niche. Cancer research. 2006;66(23):11089-93. Epub 2006/12/06. doi: 10.1158/0008-5472.Can-06-2407. PubMed PMID: 17145848; PubMed Central PMCID: PMCPMC2952469.

68. Cai B, Liao K, Song XQ, Wei WY, Zhuang Y, Zhang S. Patients with chronically diseased livers have lower incidence of colorectal liver metastases: a meta-analysis. PloS one. 
2014;9(9):e108618. Epub 2014/09/30. doi: 10.1371/journal.pone.0108618. PubMed PMID: 25265536; PubMed Central PMCID: PMCPMC4181318.

69. Giannelli G, Quaranta V, Antonaci S. Tissue remodelling in liver diseases. Histology and histopathology. 2003;18(4):1267-74. Epub 2003/09/16. doi: 10.14670/hh-18.1267. PubMed PMID: 12973693.

70. Kawaratani H, Moriya K, Namisaki T, Uejima M, Kitade M, Takeda K, et al. Therapeutic strategies for alcoholic liver disease: Focusing on inflammation and fibrosis (Review). International journal of molecular medicine. 2017;40(2):263-70. Epub 2017/06/20. doi: 10.3892/ijmm.2017.3015. PubMed PMID: 28627645.

71. Rosenow F, Ossig R, Thormeyer D, Gasmann P, Schluter K, Brunner G, et al. Integrins as antimetastatic targets of RGD-independent snake venom components in liver metastasis [corrected]. Neoplasia (New York, NY). 2008;10(2):168-76. Epub 2008/02/20. PubMed PMID: 18283339; PubMed Central PMCID: PMCPMC2244692.

72. Marcinkiewicz C. Applications of snake venom components to modulate integrin activities in cell-matrix interactions. The international journal of biochemistry \& cell biology. 2013;45(9):1974-86. Epub 2013/07/03. doi: 10.1016/j.biocel.2013.06.009. PubMed PMID: 23811033; PubMed Central PMCID: PMCPMC3774133.

73. Takada Y, Ye X, Simon S. The integrins. Genome Biology. 2007;8(5):215. doi: 10.1186/gb2007-8-5-215.

74. Rippa AL, Vorotelyak EA, Vasiliev AV, Terskikh VV. The role of integrins in the development and homeostasis of the epidermis and skin appendages. Acta naturae. 2013;5(4):22-33. Epub 2014/01/24. PubMed PMID: 24455180; PubMed Central PMCID: PMCPMC3890986.

75. Ley K, Rivera-Nieves J, Sandborn WJ, Shattil S. Integrin-based therapeutics: biological basis, clinical use and new drugs. Nature reviews Drug discovery. 2016;15(3):173-83. Epub 2016/01/30. doi: 10.1038/nrd.2015.10. PubMed PMID: 26822833; PubMed Central PMCID: PMCPMC4890615.

76. Krenkel O, Tacke F. Liver macrophages in tissue homeostasis and disease. Nature reviews Immunology. 2017;17(5):306-21. Epub 2017/03/21. doi: 10.1038/nri.2017.11. PubMed PMID: 28317925.

77. Avadanei ER, Wierzbicki PM, Giusca SE, Grigoras A, Amalinei C, Caruntu ID. Macrophage profile in primary versus secondary liver tumors. Folia histochemica et cytobiologica. 2014;52(2):112-23. Epub 2014/07/10. doi: 10.5603/fhc.2014.0014. PubMed PMID: 25007179. 78. Kim J. Regulation of Immune Cell Functions by Metabolic Reprogramming \%J Journal of Immunology Research. 2018;2018:12. doi: 10.1155/2018/8605471.

79. Powell DR, Huttenlocher A. Neutrophils in the Tumor Microenvironment. Trends in immunology. 2016;37(1):41-52. Epub 2015/12/25. doi: 10.1016/j.it.2015.11.008. PubMed PMID: 26700397; PubMed Central PMCID: PMCPMC4707100.

80. Bremnes RM, Al-Shibli K, Donnem T, Sirera R, Al-Saad S, Andersen S, et al. The role of tumor-infiltrating immune cells and chronic inflammation at the tumor site on cancer development, progression, and prognosis: emphasis on non-small cell lung cancer. Journal of thoracic oncology : official publication of the International Association for the Study of Lung Cancer. 2011;6(4):824-33. Epub 2010/12/22. doi: 10.1097/JT0.0b013e3182037b76. PubMed PMID: 21173711.

81. Treffers LW, Hiemstra IH, Kuijpers TW, van den Berg TK, Matlung HL. Neutrophils in cancer. Immunological reviews. 2016;273(1):312-28. Epub 2016/08/26. doi: 10.1111/imr.12444. PubMed PMID: 27558343. 
82. Gajewski TF, Schreiber H, Fu YX. Innate and adaptive immune cells in the tumor microenvironment. Nature immunology. 2013;14(10):1014-22. Epub 2013/09/21. doi: 10.1038/ni.2703. PubMed PMID: 24048123; PubMed Central PMCID: PMCPMC4118725. 83. Choi J, Gyamfi J, Jang H, Koo JS. The role of tumor-associated macrophage in breast cancer biology. Histology and histopathology. 2018;33(2):133-45. Epub 2017/07/07. doi: 10.14670/hh-11-916. PubMed PMID: 28681373.

84. Biswas Subhra K. Metabolic Reprogramming of Immune Cells in Cancer Progression. Immunity. 2015;43(3):435-49. doi: https://doi.org/10.1016/j.immuni.2015.09.001.

85. Balkwill FR, Capasso M, Hagemann T. The tumor microenvironment at a glance. Journal of cell science. 2012;125(Pt 23):5591-6. Epub 2013/02/20. doi: 10.1242/jcs.116392. PubMed PMID: 23420197.

86. Coussens LM, Zitvogel L, Palucka AK. Neutralizing Tumor-Promoting Chronic Inflammation: A Magic Bullet? Science. 2013;339(6117):286.

87. Dehne N, Mora J, Namgaladze D, Weigert A, Brune B. Cancer cell and macrophage crosstalk in the tumor microenvironment. Current opinion in pharmacology. 2017;35:12-9. Epub 2017/05/26. doi: 10.1016/j.coph.2017.04.007. PubMed PMID: 28538141.

88. Noy R, Pollard JW. Tumor-associated macrophages: from mechanisms to therapy. Immunity. 2014;41(1):49-61. Epub 2014/07/19. doi: 10.1016/j.immuni.2014.06.010. PubMed PMID: 25035953; PubMed Central PMCID: PMCPMC4137410.

89. Ramanathan S, Jagannathan N. Tumor associated macrophage: a review on the phenotypes, traits and functions. Iranian journal of cancer prevention. 2014;7(1):1-8. Epub 2014/09/25. PubMed PMID: 25250141; PubMed Central PMCID: PMCPMC4142950.

90. Lewis CE, Pollard JW. Distinct role of macrophages in different tumor microenvironments. Cancer research. 2006;66(2):605-12. Epub 2006/01/21. doi: 10.1158/00085472.Can-05-4005. PubMed PMID: 16423985.

91. Brady NJ, Chuntova P, Schwertfeger KL. Macrophages: Regulators of the Inflammatory Microenvironment during Mammary Gland Development and Breast Cancer. Mediators of inflammation. 2016;2016:4549676. Epub 2016/02/18. doi: 10.1155/2016/4549676. PubMed PMID: 26884646; PubMed Central PMCID: PMCPMC4739263.

92. Shen L, Li H, Shi Y, Wang D, Gong J, Xun J, et al. M2 tumour-associated macrophages contribute to tumour progression via legumain remodelling the extracellular matrix in diffuse large B cell lymphoma. Sci Rep. 2016;6:30347. Epub 2016/07/29. doi: 10.1038/srep30347. PubMed PMID: 27464733; PubMed Central PMCID: PMCPMC4964568.

93. Zhong X, Chen B, Yang Z. The Role of Tumor-Associated Macrophages in Colorectal Carcinoma Progression. Cellular physiology and biochemistry : international journal of experimental cellular physiology, biochemistry, and pharmacology. 2018;45(1):356-65. Epub 2018/02/07. doi: 10.1159/000486816. PubMed PMID: 29402795.

94. Erreni M, Mantovani A, Allavena P. Tumor-associated Macrophages (TAM) and Inflammation in Colorectal Cancer. Cancer microenvironment : official journal of the International Cancer Microenvironment Society. 2011;4(2):141-54. Epub 2011/09/13. doi: 10.1007/s12307-010-0052-5. PubMed PMID: 21909876; PubMed Central PMCID: PMCPMC3170420.

95. Capece D, Fischietti M, Verzella D, Gaggiano A, Cicciarelli G, Tessitore A, et al. The inflammatory microenvironment in hepatocellular carcinoma: a pivotal role for tumorassociated macrophages. BioMed research international. 2013;2013:187204. Epub 2013/03/28. doi: 10.1155/2013/187204. PubMed PMID: 23533994; PubMed Central PMCID: PMCPMC3591180. 
96. Lin CC, Hsu C, Hsu CH, Hsu WL, Cheng AL, Yang CH. Arsenic trioxide in patients with hepatocellular carcinoma: a phase II trial. Investigational new drugs. 2007;25(1):77-84. Epub 2006/08/29. doi: 10.1007/s10637-006-9004-9. PubMed PMID: 16937079.

97. Choi K, Medley, J.K., Konig, M., Stocking, K., Cannistra, C. Tellurium, version 2.3.5.2 [computer program]. Washington University of Washington; 2014.

98. Beier JI, Luyendyk JP, Guo L, von Montfort C, Staunton DE, Arteel GE. Fibrin accumulation plays a critical role in the sensitization to lipopolysaccharide-induced liver injury caused by ethanol in mice. Hepatology (Baltimore, Md). 2009;49(5):1545-53. Epub 2009/03/18. doi: 10.1002/hep.22847. PubMed PMID: 19291788; PubMed Central PMCID: PMCPMC2852109. 99. Falcon S, Carey, V. Settles, M., de Beuf, K., Carvalho, B. pdInfoBuilder Platform Design Information Package Builder. R package version 1.46 .0 ed2018.

100. Ritchie ME, Phipson B, Wu D, Hu Y, Law CW, Shi W, et al. limma powers differential expression analyses for RNA-sequencing and microarray studies. Nucleic acids research. 2015;43(7):e47. Epub 2015/01/22. doi: 10.1093/nar/gkv007. PubMed PMID: 25605792; PubMed Central PMCID: PMCPMC4402510.

101. Segata N, Izard J, Waldron L, Gevers D, Miropolsky L, Garrett WS, et al. Metagenomic biomarker discovery and explanation. Genome Biol. 2011;12(6):R60. Epub 2011/06/28. doi: 10.1186/gb-2011-12-6-r60. PubMed PMID: 21702898; PubMed Central PMCID: PMCPMC3218848.

102. Agarwal V, Bell GW, Nam JW, Bartel DP. Predicting effective microRNA target sites in mammalian mRNAs. eLife. 2015;4. Epub 2015/08/13. doi: 10.7554/eLife.05005. PubMed PMID: 26267216; PubMed Central PMCID: PMCPMC4532895.

103. Griffiths-Jones S, Grocock RJ, van Dongen S, Bateman A, Enright AJ. miRBase: microRNA sequences, targets and gene nomenclature. Nucleic acids research. 2006;34(Database issue):D140-4. Epub 2005/12/31. doi: 10.1093/nar/gkj112. PubMed PMID: 16381832; PubMed Central PMCID: PMCPMC1347474.

104. Griffiths-Jones S, Saini HK, van Dongen S, Enright AJ. miRBase: tools for microRNA genomics. Nucleic acids research. 2008;36(Database issue):D154-8. Epub 2007/11/10. doi: 10.1093/nar/gkm952. PubMed PMID: 17991681; PubMed Central PMCID: PMCPMC2238936. 105. Kozomara A, Griffiths-Jones S. miRBase: annotating high confidence microRNAs using deep sequencing data. Nucleic acids research. 2014;42(Database issue):D68-73. Epub 2013/11/28. doi: 10.1093/nar/gkt1181. PubMed PMID: 24275495; PubMed Central PMCID: PMCPMC3965103.

106. Winslow MM, Dayton TL, Verhaak RG, Kim-Kiselak C, Snyder EL, Feldser DM, et al. Suppression of lung adenocarcinoma progression by Nkx2-1. Nature. 2011;473(7345):101-4. Epub 2011/04/08. doi: 10.1038/nature09881. PubMed PMID: 21471965; PubMed Central PMCID: PMCPMC3088778.

107. Hynes RO. The extracellular matrix: not just pretty fibrils. Science. 2009;326(5957):12169. Epub 2009/12/08. doi: 10.1126/science.1176009. PubMed PMID: 19965464; PubMed Central PMCID: PMCPMC3536535.

108. Hynes RO. Integrins: a family of cell surface receptors. Cell. 1987;48(4):549-54. Epub 1987/02/27. PubMed PMID: 3028640.

109. Hynes RO. The emergence of integrins: a personal and historical perspective. Matrix Biol. 2004;23(6):333-40. Epub 2004/11/10. doi: 10.1016/j.matbio.2004.08.001. PubMed PMID: 15533754; PubMed Central PMCID: PMCPMC3493146.

110. Patsenker E, Stickel F. Role of integrins in fibrosing liver diseases. American journal of physiology Gastrointestinal and liver physiology. 2011;301(3):G425-34. Epub 2011/06/11. doi: 10.1152/ajpgi.00050.2011. PubMed PMID: 21659620. 
111. Masson-Gadais B, Pierres A, Benoliel AM, Bongrand P, Lissitzky JC. Integrin (alpha) and beta subunit contribution to the kinetic properties of (alpha)2beta1 collagen receptors on human keratinocytes analyzed under hydrodynamic conditions. Journal of cell science. 1999;112 ( Pt 14):2335-45. Epub 1999/06/25. PubMed PMID: 10381389.

112. Legate KR, Wickstrom SA, Fassler R. Genetic and cell biological analysis of integrin outside-in signaling. Genes \& development. 2009;23(4):397-418. Epub 2009/02/26. doi: 10.1101/gad.1758709. PubMed PMID: 19240129.

113. Pouwels J, Nevo J, Pellinen T, Ylanne J, Ivaska J. Negative regulators of integrin activity. Journal of cell science. 2012;125(Pt 14):3271-80. Epub 2012/07/24. doi: 10.1242/jcs.093641. PubMed PMID: 22822081.

114. Zhao T, Li Y, Dinner AR. How focal adhesion size depends on integrin affinity. Langmuir : the ACS journal of surfaces and colloids. 2009;25(3):1540-6. Epub 2009/01/10. doi: 10.1021/la8026804. PubMed PMID: 19132823.

115. Plow EF, Haas TA, Zhang L, Loftus J, Smith JW. Ligand binding to integrins. The Journal of biological chemistry. 2000;275(29):21785-8. Epub 2000/05/10. doi: 10.1074/jbc.R000003200. PubMed PMID: 10801897.

116. Mallet DG, Pettet GJ. A mathematical model of integrin-mediated haptotactic cell migration. Bulletin of mathematical biology. 2006;68(2):231-53. Epub 2006/06/24. doi: 10.1007/s11538-005-9032-1. PubMed PMID: 16794929.

117. Dutta-Moscato J, Solovyev A, Mi Q, Nishikawa T, Soto-Gutierrez A, Fox IJ, et al. A Multiscale Agent-Based in silico Model of Liver Fibrosis Progression. Frontiers in bioengineering and biotechnology. 2014;2:18. Epub 2014/08/26. doi: 10.3389/fbioe.2014.00018. PubMed PMID: 25152891; PubMed Central PMCID: PMCPMC4126446.

118. Welf ES, Naik UP, Ogunnaike BA. A spatial model for integrin clustering as a result of feedback between integrin activation and integrin binding. Biophysical journal. 2012;103(6):1379-89. Epub 2012/09/22. doi: 10.1016/j.bpj.2012.08.021. PubMed PMID: 22995511; PubMed Central PMCID: PMCPMC3446678.

119. Wanant S, Quon MJ. Insulin receptor binding kinetics: modeling and simulation studies. Journal of theoretical biology. 2000;205(3):355-64. Epub 2000/07/07. doi:

10.1006/jtbi.2000.2069. PubMed PMID: 10882558.

120. Buck CA, Horwitz AF. Cell surface receptors for extracellular matrix molecules. Annual review of cell biology. 1987;3:179-205. Epub 1987/01/01. doi:

10.1146/annurev.cb.03.110187.001143. PubMed PMID: 2825736.

121. Takagi J, Strokovich K, Springer TA, Walz T. Structure of integrin alpha5beta1 in complex with fibronectin. The EMBO journal. 2003;22(18):4607-15. Epub 2003/09/13. doi:

10.1093/emboj/cdg445. PubMed PMID: 12970173; PubMed Central PMCID: PMCPMC212714.

122. Duncan MB, Yang C, Tanjore H, Boyle PM, Keskin D, Sugimoto H, et al. Type XVIII collagen is essential for survival during acute liver injury in mice. Disease models \& mechanisms. 2013;6(4):942-51. Epub 2013/04/13. doi: 10.1242/dmm.011577. PubMed PMID: 23580202; PubMed Central PMCID: PMCPMC3701214.

123. Jiang Y, Liu, J., Waalkes, M., Kang, Y.J. Changes in the gene expression associated with cabon tetrachloride-induced liver fibrosis persist after cessation of dosing in mice. . Toxicological Sci. 2004;79:404-10.

124. Wisniewski JR, Hein MY, Cox J, Mann M. A "proteomic ruler" for protein copy number and concentration estimation without spike-in standards. Molecular \& cellular proteomics : MCP. 2014;13(12):3497-506. Epub 2014/09/17. doi: 10.1074/mcp.M113.037309. PubMed PMID: 25225357; PubMed Central PMCID: PMCPMC4256500. 
125. Ishihama Y, Oda Y, Tabata T, Sato T, Nagasu T, Rappsilber J, et al. Exponentially modified protein abundance index (emPAl) for estimation of absolute protein amount in proteomics by the number of sequenced peptides per protein. Molecular \& cellular proteomics : MCP. 2005;4(9):1265-72. Epub 2005/06/17. doi: 10.1074/mcp.M500061-MCP200. PubMed PMID: 15958392.

126. Kislinger T, Emili A. Multidimensional protein identification technology: current status and future prospects. Expert review of proteomics. 2005;2(1):27-39. Epub 2005/06/22. doi: 10.1586/14789450.2.1.27. PubMed PMID: 15966850.

127. Schirmer EC, Yates JR, 3rd, Gerace L. MudPIT: A powerful proteomics tool for discovery. Discovery medicine. 2003;3(18):38-9. Epub 2003/10/01. PubMed PMID: 20704860.

128. Kim JK, Xu Y, Xu X, Keene DR, Gurusiddappa S, Liang X, et al. A novel binding site in collagen type III for integrins alpha1beta1 and alpha2beta1. The Journal of biological chemistry. 2005;280(37):32512-20. Epub 2005/07/27. doi: 10.1074/jbc.M502431200. PubMed PMID: 16043429.

129. Xu Y, Gurusiddappa S, Rich RL, Owens RT, Keene DR, Mayne R, et al. Multiple binding sites in collagen type I for the integrins alpha1beta1 and alpha2beta1. The Journal of biological chemistry. 2000;275(50):38981-9. Epub 2000/09/15. doi: 10.1074/jbc.M007668200. PubMed PMID: 10986291.

130. Taubenberger A, Cisneros DA, Friedrichs J, Puech PH, Muller DJ, Franz CM. Revealing early steps of alpha2beta1 integrin-mediated adhesion to collagen type I by using single-cell force spectroscopy. Molecular biology of the cell. 2007;18(5):1634-44. Epub 2007/02/23. doi: 10.1091/mbc.e06-09-0777. PubMed PMID: 17314408; PubMed Central PMCID: PMCPMC1855039.

131. Kern A, Eble J, Golbik R, Kuhn K. Interaction of type IV collagen with the isolated integrins alpha 1 beta 1 and alpha 2 beta 1 . European journal of biochemistry. 1993;215(1):1519. Epub 1993/07/01. PubMed PMID: 8344274.

132. Hantgan RR, Stahle MC, Lord ST. Dynamic regulation of fibrinogen: integrin alphallbbeta3 binding. Biochemistry. 2010;49(43):9217-25. Epub 2010/09/11. doi: 10.1021/bi1009858. PubMed PMID: 20828133; PubMed Central PMCID: PMCPMC3210020. 133. Brown AC, Dysart MM, Clarke KC, Stabenfeldt SE, Barker TH. Integrin alpha3beta1 Binding to Fibronectin Is Dependent on the Ninth Type III Repeat. The Journal of biological chemistry. 2015;290(42):25534-47. Epub 2015/09/01. doi: 10.1074/jbc.M115.656702. PubMed PMID: 26318455; PubMed Central PMCID: PMCPMC4646199.

134. Denis C, Williams JA, Lu X, Meyer D, Baruch D. Solid-phase von Willebrand factor contains a conformationally active RGD motif that mediates endothelial cell adhesion through the alpha v beta 3 receptor. Blood. 1993;82(12):3622-30. Epub 1993/12/15. PubMed PMID: 7505120.

135. Peerschke El, Francis CW, Marder VJ. Fibrinogen binding to human blood platelets: effect of gamma chain carboxyterminal structure and length. Blood. 1986;67(2):385-90. Epub 1986/02/01. PubMed PMID: 3942829.

136. Ware S, Donahue JP, Hawiger J, Anderson WF. Structure of the fibrinogen gamma-chain integrin binding and factor XIIla cross-linking sites obtained through carrier protein driven crystallization. Protein science : a publication of the Protein Society. 1999;8(12):2663-71. Epub 2000/01/13. doi: 10.1110/ps.8.12.2663. PubMed PMID: 10631982; PubMed Central PMCID: PMCPMC2144222.

137. Bruck R, Hershkoviz R, Lider O, Shirin H, Aeed H, Halpern Z. The use of synthetic analogues of Arg-Gly-Asp (RGD) and soluble receptor of tumor necrosis factor to prevent acute 
and chronic experimental liver injury. The Yale journal of biology and medicine. 1997;70(4):391402. Epub 1997/07/01. PubMed PMID: 9626759; PubMed Central PMCID: PMCPMC2589337.

138. Care BR, Soula HA. Impact of receptor clustering on ligand binding. BMC systems biology. 2011;5:48. Epub 2011/04/02. doi: 10.1186/1752-0509-5-48. PubMed PMID: 21453460; PubMed Central PMCID: PMCPMC3082237.

139. Nieto N, Lutolf MP. Extracellular matrix bioengineering and systems biology approaches in liver disease. Systems and synthetic biology. 2011;5(1-2):11-20. Epub 2012/06/02. doi: 10.1007/s11693-011-9085-4. PubMed PMID: 22654992; PubMed Central PMCID: PMCPMC3159691.

140. Delire B, Starkel P, Leclercq I. Animal Models for Fibrotic Liver Diseases: What We Have, What We Need, and What Is under Development. Journal of clinical and translational hepatology. 2015;3(1):53-66. Epub 2015/09/12. doi: 10.14218/jcth.2014.00035. PubMed PMID: 26357635; PubMed Central PMCID: PMCPMC4542084.

141. Liedtke C, Luedde T, Sauerbruch T, Scholten D, Streetz K, Tacke F, et al. Experimental liver fibrosis research: update on animal models, legal issues and translational aspects.

Fibrogenesis \& tissue repair. 2013;6(1):19. Epub 2013/11/28. doi: 10.1186/1755-1536-6-19. PubMed PMID: 24274743; PubMed Central PMCID: PMCPMC3850878.

142. Shimaoka M, Springer TA. Therapeutic antagonists and conformational regulation of integrin function. Nature reviews Drug discovery. 2003;2(9):703-16. Epub 2003/09/03. doi: 10.1038/nrd1174. PubMed PMID: 12951577.

143. Henderson NC, Arnold TD, Katamura Y, Giacomini MM, Rodriguez JD, McCarty JH, et al. Targeting of alphav integrin identifies a core molecular pathway that regulates fibrosis in several organs. Nature medicine. 2013;19(12):1617-24. Epub 2013/11/13. doi: 10.1038/nm.3282. PubMed PMID: 24216753; PubMed Central PMCID: PMCPMC3855865.

144. Agarwal SK. Integrins and cadherins as therapeutic targets in fibrosis. Frontiers in pharmacology. 2014;5:131. Epub 2014/06/12. doi: 10.3389/fphar.2014.00131. PubMed PMID: 24917820; PubMed Central PMCID: PMCPMC4042084.

145. Millard M, Odde S, Neamati N. Integrin targeted therapeutics. Theranostics. 2011;1:15488. Epub 2011/05/07. PubMed PMID: 21547158; PubMed Central PMCID: PMCPMC3086618.

146. Goodman SL, Picard M. Integrins as therapeutic targets. Trends in pharmacological sciences. 2012;33(7):405-12. Epub 2012/05/29. doi: 10.1016/j.tips.2012.04.002. PubMed PMID: 22633092.

147. National Research Council Subcommittee on Arsenic in Drinking W. Arsenic in Drinking Water. Washington (DC): National Academies Press (US)

Copyright 1999 by the National Academy of Sciences. All rights reserved.; 1999.

148. National Research Council Subcommittee to Update the Arsenic in Drinking Water R. Arsenic in Drinking Water: 2001 Update. Washington (DC): National Academies Press (US)

Copyright 2001 by the National Academy of Sciences. All rights reserved.; 2001.

149. Frost FJ, Muller T, Petersen HV, Thomson B, Tollestrup K. Identifying US populations for the study of health effects related to drinking water arsenic. Journal Of Exposure Analysis And Environmental Epidemiology. 2003;13:231. doi: 10.1038/sj.jea.7500275.

150. Engel RR, Smith AH. Arsenic in drinking water and mortality from vascular disease: an ecologic analysis in 30 counties in the United States. Archives of environmental health. 1994;49(5):418-27. Epub 1994/09/01. doi: 10.1080/00039896.1994.9954996. PubMed PMID: 7944575.

151. Zheng Y, Ayotte JD. At the crossroads: Hazard assessment and reduction of health risks from arsenic in private well waters of the northeastern United States and Atlantic Canada. The 
Science of the total environment. 2015;505:1237-47. Epub 2014/12/04. doi: 10.1016/j.scitotenv.2014.10.089. PubMed PMID: 25466685; PubMed Central PMCID: PMCPMC4386837.

152. Waalkes MP, Liu J, Chen H, Xie Y, Achanzar WE, Zhou YS, et al. Estrogen signaling in livers of male mice with hepatocellular carcinoma induced by exposure to arsenic in utero. Journal of the National Cancer Institute. 2004;96(6):466-74. Epub 2004/03/18. PubMed PMID: 15026472.

153. Birnbaum LS, Jung P. From endocrine disruptors to nanomaterials: advancing our understanding of environmental health to protect public health. Health affairs (Project Hope). 2011;30(5):814-22. Epub 2011/05/11. doi: 10.1377/h/thaff.2010.1225. PubMed PMID: 21555467.

154. Arteel GE, Guo L, Schlierf T, Beier JI, Kaiser JP, Chen TS, et al. Subhepatotoxic exposure to arsenic enhances lipopolysaccharide-induced liver injury in mice. Toxicology and applied pharmacology. 2008;226(2):128-39. Epub 2007/10/09. doi: 10.1016/j.taap.2007.08.020. PubMed PMID: 17919673; PubMed Central PMCID: PMCPMC2246049.

155. Tan M, Schmidt RH, Beier JI, Watson WH, Zhong H, States JC, et al. Chronic subhepatotoxic exposure to arsenic enhances hepatic injury caused by high fat diet in mice. Toxicology and applied pharmacology. 2011;257(3):356-64. Epub 2011/10/11. doi: 10.1016/j.taap.2011.09.019. PubMed PMID: 21983427; PubMed Central PMCID: PMCPMC3232462.

156. Zierold KM, Knobeloch L, Anderson H. Prevalence of chronic diseases in adults exposed to arsenic-contaminated drinking water. American journal of public health. 2004;94(11):1936-7. Epub 2004/10/30. PubMed PMID: 15514231; PubMed Central PMCID: PMCPMC1448563. 157. Wu Y, Zhou BP. Inflammation: a driving force speeds cancer metastasis. Cell cycle (Georgetown, Tex). 2009;8(20):3267-73. Epub 2009/09/23. doi: 10.4161/cc.8.20.9699. PubMed PMID: 19770594; PubMed Central PMCID: PMCPMC3702728.

158. Domingueti CP, Dusse LM, Carvalho M, de Sousa LP, Gomes KB, Fernandes AP. Diabetes mellitus: The linkage between oxidative stress, inflammation, hypercoagulability and vascular complications. Journal of diabetes and its complications. 2016;30(4):738-45. Epub 2016/01/20. doi: 10.1016/j.jdiacomp.2015.12.018. PubMed PMID: 26781070.

159. Selgrade MK. Immunotoxicity: the risk is real. Toxicological sciences : an official journal of the Society of Toxicology. 2007;100(2):328-32. Epub 2007/09/20. doi: 10.1093/toxsci/kfm244. PubMed PMID: 17878151.

160. Lantz RC, Parliman G, Chen GJ, Carter DE. Effect of Arsenic Exposure on Alveolar Macrophage Function: I. Effect of Soluble As(III) and As(V). Environmental Research. 1994;67(2):183-95. doi: https://doi.org/10.1006/enrs.1994.1073.

161. Wu MM, Chiou HY, Ho IC, Chen CJ, Lee TC. Gene expression of inflammatory molecules in circulating lymphocytes from arsenic-exposed human subjects. Environmental health perspectives. 2003;111(11):1429-38. Epub 2003/08/21. doi: 10.1289/ehp.6396. PubMed PMID: $12928151 ;$ PubMed Central PMCID: PMCPMC1241636.

162. Sakurai T, Ohta T, Tomita N, Kojima C, Hariya Y, Mizukami A, et al. Evaluation of immunotoxic and immunodisruptive effects of inorganic arsenite on human monocytes/macrophages. International immunopharmacology. 2006;6(2):304-15. Epub 2006/02/08. PubMed PMID: 16459422.

163. Aranyi C, Bradof JN, O'Shea WJ, Graham JA, Miller FJ. Effects of arsenic trioxide inhalation exposure on pulmonary antibacterial defenses in mice. Journal of toxicology and environmental health. 1985;15(1):163-72. Epub 1985/01/01. doi: 10.1080/15287398509530643. PubMed PMID: 3884825. 
164. Takahashi M, Ota A, Karnan S, Hossain E, Konishi Y, Damdindorj L, et al. Arsenic trioxide prevents nitric oxide production in lipopolysaccharide -stimulated RAW 264.7 by inhibiting a TRIF-dependent pathway. Cancer science. 2013;104(2):165-70. Epub 2012/10/31. doi:

10.1111/cas.12053. PubMed PMID: 23106696.

165. Padovani AM, Molina MF, Mann KK. Inhibition of liver $x$ receptor/retinoid X receptormediated transcription contributes to the proatherogenic effects of arsenic in macrophages in vitro. Arteriosclerosis, thrombosis, and vascular biology. 2010;30(6):1228-36. Epub 2010/03/27. doi: 10.1161/atvbaha.110.205500. PubMed PMID: 20339114.

166. Cui J, Xu W, Chen J, Li H, Dai L, Frank JA, et al. M2 polarization of macrophages facilitates arsenic-induced cell transformation of lung epithelial cells. Oncotarget. 2017;8(13):21398-409. Epub 2017/04/21. doi: 10.18632/oncotarget.15232. PubMed PMID: 28423485; PubMed Central PMCID: PMCPMC5400592.

167. Vandanmagsar B, Youm YH, Ravussin A, Galgani JE, Stadler K, Mynatt RL, et al. The NLRP3 inflammasome instigates obesity-induced inflammation and insulin resistance. Nature medicine. 2011;17(2):179-88. Epub 2011/01/11. doi: 10.1038/nm.2279. PubMed PMID: 21217695; PubMed Central PMCID: PMCPMC3076025.

168. Melton DW, Lei X, Gelfond JA, Shireman PK. Dynamic macrophage polarization-specific miRNA patterns reveal increased soluble VEGF receptor 1 by miR-125a-5p inhibition. Physiological genomics. 2016;48(5):345-60. Epub 2016/02/18. doi: 10.1152/physiolgenomics.00098.2015. PubMed PMID: 26884460; PubMed Central PMCID: PMCPMC4855214.

169. Graff JW, Dickson AM, Clay G, McCaffrey AP, Wilson ME. Identifying functional microRNAs in macrophages with polarized phenotypes. The Journal of biological chemistry. 2012;287(26):21816-25. Epub 2012/05/03. doi: 10.1074/jbc.M111.327031. PubMed PMID: 22549785; PubMed Central PMCID: PMCPMC3381144.

170. Quinn SR, O'Neill LA. A trio of microRNAs that control Toll-like receptor signalling. International immunology. 2011;23(7):421-5. Epub 2011/06/10. doi: 10.1093/intimm/dxr034. PubMed PMID: 21652514.

171. Liu G, Abraham E. MicroRNAs in immune response and macrophage polarization. Arteriosclerosis, thrombosis, and vascular biology. 2013;33(2):170-7. Epub 2013/01/18. doi: 10.1161/atvbaha.112.300068. PubMed PMID: 23325473; PubMed Central PMCID: PMCPMC3549532.

172. Beezhold K, Klei LR, Barchowsky A. Regulation of cyclin D1 by arsenic and microRNA inhibits adipogenesis. Toxicology letters. 2017;265:147-55. Epub 2016/12/10. doi: 10.1016/j.toxlet.2016.12.002. PubMed PMID: 27932253; PubMed Central PMCID: PMCPMC5182514.

173. Hino K, Horigome K, Nishio M, Komura S, Nagata S, Zhao C, et al. Activin-A enhances mTOR signaling to promote aberrant chondrogenesis in fibrodysplasia ossificans progressiva. The Journal of clinical investigation. 2017;127(9):3339-52. Epub 2017/08/02. doi: 10.1172/jci93521. PubMed PMID: 28758906; PubMed Central PMCID: PMCPMC5669572. 174. Martin SK, Fitter S, Dutta AK, Matthews MP, Walkley CR, Hall MN, et al. Brief report: the differential roles of mTORC1 and mTORC2 in mesenchymal stem cell differentiation. Stem cells (Dayton, Ohio). 2015;33(4):1359-65. Epub 2014/12/30. doi: 10.1002/stem.1931. PubMed PMID: 25537496.

175. Andrew AS, Jewell DA, Mason RA, Whitfield ML, Moore JH, Karagas MR. Drinking-water arsenic exposure modulates gene expression in human lymphocytes from a U.S. population. Environmental health perspectives. 2008;116(4):524-31. Epub 2008/04/17. doi: 10.1289/ehp.10861. PubMed PMID: 18414638; PubMed Central PMCID: PMCPMC2290973. 
176. Hernandez-Castro B, Doniz-Padilla LM, Salgado-Bustamante M, Rocha D, Ortiz-Perez MD, Jimenez-Capdeville ME, et al. Effect of arsenic on regulatory T cells. Journal of clinical immunology. 2009;29(4):461-9. Epub 2009/02/28. doi: 10.1007/s10875-009-9280-1. PubMed PMID: 19247822.

177. Lemarie A, Morzadec C, Bourdonnay E, Fardel O, Vernhet L. Human macrophages constitute targets for immunotoxic inorganic arsenic. Journal of immunology (Baltimore, Md : 1950). 2006;177(5):3019-27. Epub 2006/08/22. PubMed PMID: 16920938.

178. Nayak AS, Lage CR, Kim CH. Effects of low concentrations of arsenic on the innate immune system of the zebrafish (Danio rerio). Toxicological sciences : an official journal of the Society of Toxicology. 2007;98(1):118-24. Epub 2007/04/03. doi: 10.1093/toxsci/kfm072. PubMed PMID: 17400579.

179. Straub AC, Clark KA, Ross MA, Chandra AG, Li S, Gao X, et al. Arsenic-stimulated liver sinusoidal capillarization in mice requires NADPH oxidase-generated superoxide. The Journal of clinical investigation. 2008;118(12):3980-9. Epub 2008/11/27. doi: 10.1172/jci35092. PubMed PMID: 19033667; PubMed Central PMCID: PMCPMC2582440.

180. Kozul CD, Hampton TH, Davey JC, Gosse JA, Nomikos AP, Eisenhauer PL, et al. Chronic exposure to arsenic in the drinking water alters the expression of immune response genes in mouse lung. Environmental health perspectives. 2009;117(7):1108-15. Epub 2009/08/06. doi: 10.1289/ehp.0800199. PubMed PMID: 19654921; PubMed Central PMCID: PMCPMC2717138.

181. Kanters E, Pasparakis M, Gijbels MJ, Vergouwe MN, Partouns-Hendriks I, Fijneman RJ, et al. Inhibition of NF-kappaB activation in macrophages increases atherosclerosis in LDL receptordeficient mice. The Journal of clinical investigation. 2003;112(8):1176-85. Epub 2003/10/17. doi: 10.1172/jci18580. PubMed PMID: 14561702; PubMed Central PMCID: PMCPMC213488. 182. Doyle SE, O'Connell RM, Miranda GA, Vaidya SA, Chow EK, Liu PT, et al. Toll-like receptors induce a phagocytic gene program through $\mathrm{p} 38$. The Journal of experimental medicine. 2004;199(1):81-90. Epub 2003/12/31. doi: 10.1084/jem.20031237. PubMed PMID: 14699082; PubMed Central PMCID: PMCPMC1887723.

183. Cho YJ, Cunnick JM, Yi SJ, Kaartinen V, Groffen J, Heisterkamp N. Abr and Bcr, two homologous Rac GTPase-activating proteins, control multiple cellular functions of murine macrophages. Molecular and cellular biology. 2007;27(3):899-911. Epub 2006/11/23. doi: 10.1128/mcb.00756-06. PubMed PMID: 17116687; PubMed Central PMCID: PMCPMC1800684. 184. Weischenfeldt J, Porse B. Bone Marrow-Derived Macrophages (BMM): Isolation and Applications. CSH protocols. 2008;2008:pdb.prot5080. Epub 2008/01/01. doi: 10.1101/pdb.prot5080. PubMed PMID: 21356739.

185. Davis MJ, Tsang TM, Qiu Y, Dayrit JK, Freij JB, Huffnagle GB, et al. Macrophage M1/M2 polarization dynamically adapts to changes in cytokine microenvironments in Cryptococcus neoformans infection. mBio. 2013;4(3):e00264-13. Epub 2013/06/20. doi: 10.1128/mBio.0026413. PubMed PMID: 23781069; PubMed Central PMCID: PMCPMC3684832.

186. O'Neill LA, Sheedy FJ, McCoy CE. MicroRNAs: the fine-tuners of Toll-like receptor signalling. Nature reviews Immunology. 2011;11(3):163-75. Epub 2011/02/19. doi: 10.1038/nri2957. PubMed PMID: 21331081.

187. Xu L, Yang F, Lin R, Han C, Liu J, Ding Z. Induction of $\mathrm{m} 2$ polarization in primary culture liver macrophages from rats with acute pancreatitis. PloS one. 2014;9(9):e108014. Epub 2014/09/27. doi: 10.1371/journal.pone.0108014. PubMed PMID: 25259888; PubMed Central PMCID: PMCPMC4178066.

188. Sica A, Invernizzi P, Mantovani A. Macrophage plasticity and polarization in liver homeostasis and pathology. Hepatology (Baltimore, Md). 2014;59(5):2034-42. Epub 2013/10/12. doi: 10.1002/hep.26754. PubMed PMID: 24115204. 
189. Alvarez MM, Liu JC, Trujillo-de Santiago G, Cha BH, Vishwakarma A, Ghaemmaghami $\mathrm{AM}$, et al. Delivery strategies to control inflammatory response: Modulating M1-M2 polarization in tissue engineering applications. Journal of controlled release : official journal of the Controlled Release Society. 2016;240:349-63. Epub 2016/01/19. doi: 10.1016/j.jconrel.2016.01.026. PubMed PMID: 26778695; PubMed Central PMCID: PMCPMC4945478.

190. Xue J, Schmidt SV, Sander J, Draffehn A, Krebs W, Quester I, et al. Transcriptome-based network analysis reveals a spectrum model of human macrophage activation. Immunity. 2014;40(2):274-88. Epub 2014/02/18. doi: 10.1016/j.immuni.2014.01.006. PubMed PMID: 24530056; PubMed Central PMCID: PMCPMC3991396.

191. Sengupta M, Bishayi B. Effect of lead and arsenic on murine macrophage response. Drug and chemical toxicology. 2002;25(4):459-72. Epub 2002/10/16. doi: 10.1081/dct-120014796. PubMed PMID: 12378952.

192. Bishayi B, Sengupta M. Intracellular survival of Staphylococcus aureus due to alteration of cellular activity in arsenic and lead intoxicated mature Swiss albino mice. Toxicology. 2003;184(1):31-9. Epub 2002/12/31. PubMed PMID: 12505374.

193. Martinez FO, Gordon S. The M1 and M2 paradigm of macrophage activation: time for reassessment. F1000prime reports. 2014;6:13. Epub 2014/03/29. doi: 10.12703/p6-13. PubMed PMID: 24669294; PubMed Central PMCID: PMCPMC3944738.

194. Hatch GE, Boykin E, Graham JA, Lewtas J, Pott F, Loud K, et al. Inhalable particles and pulmonary host defense: in vivo and in vitro effects of ambient air and combustion particles. Environ Res. 1985;36(1):67-80. Epub 1985/02/01. PubMed PMID: 3967645.

195. Kim DH, Saetrom P, Snove O, Jr., Rossi JJ. MicroRNA-directed transcriptional gene silencing in mammalian cells. Proceedings of the National Academy of Sciences of the United States of America. 2008;105(42):16230-5. Epub 2008/10/15. doi: 10.1073/pnas.0808830105. PubMed PMID: 18852463; PubMed Central PMCID: PMCPMC2571020.

196. Chavali S, Bruhn S, Tiemann K, Saetrom P, Barrenas F, Saito T, et al. MicroRNAs act complementarily to regulate disease-related mRNA modules in human diseases. RNA (New York, NY). 2013;19(11):1552-62. Epub 2013/09/26. doi: 10.1261/rna.038414.113. PubMed PMID:

24062574 ; PubMed Central PMCID: PMCPMC3851722.

197. Barton GM. A calculated response: control of inflammation by the innate immune system. The Journal of clinical investigation. 2008;118(2):413-20. Epub 2008/02/05. doi: 10.1172/jci34431. PubMed PMID: 18246191; PubMed Central PMCID: PMCPMC2214713. 198. Chiu CC, Wu WS. Investigation of microRNAs in mouse macrophage responses to lipopolysaccharide-stimulation by combining gene expression with microRNA-target information. BMC genomics. 2015;16 Suppl 12:S13. Epub 2015/12/19. doi: 10.1186/1471-216416-s12-s13. PubMed PMID: 26680554; PubMed Central PMCID: PMCPMC4682375.

199. McCoy CE, Sheedy FJ, Qualls JE, Doyle SL, Quinn SR, Murray PJ, et al. IL-10 inhibits miR155 induction by toll-like receptors. The Journal of biological chemistry. 2010;285(27):20492-8. Epub 2010/05/04. doi: 10.1074/jbc.M110.102111. PubMed PMID: 20435894; PubMed Central PMCID: PMCPMC2898307.

200. Muto T, Varney M, Walker C, Bolanos L, Hueneman K, Starczynowski D. Innate Immune Signal Pathway Activation Induced By Combined Loss of Del(5q) MDS Genes, Tifab and Mir-146a, Accelerates Hematopoietic Stem Cell Exhaustion Driven By Chronic Exposure to a TLR Ligands. Blood. 2017;130(Suppl 1):1674.

201. Androulidaki A, lliopoulos D, Arranz A, Doxaki C, Schworer S, Zacharioudaki V, et al. The kinase Akt1 controls macrophage response to lipopolysaccharide by regulating microRNAs. Immunity. 2009;31(2):220-31. Epub 2009/08/25. doi: 10.1016/j.immuni.2009.06.024. PubMed PMID: 19699171; PubMed Central PMCID: PMCPMC2865583. 
202. Liu G, Friggeri A, Yang Y, Park YJ, Tsuruta Y, Abraham E. miR-147, a microRNA that is induced upon Toll-like receptor stimulation, regulates murine macrophage inflammatory responses. Proceedings of the National Academy of Sciences of the United States of America. 2009;106(37):15819-24. Epub 2009/09/02. doi: 10.1073/pnas.0901216106. PubMed PMID: 19721002; PubMed Central PMCID: PMCPMC2747202.

203. Yeruva L, Pouncey DL, Eledge MR, Bhattacharya S, Luo C, Weatherford EW, et al. MicroRNAs Modulate Pathogenesis Resulting from Chlamydial Infection in Mice. Infection and immunity. 2017;85(1). Epub 2016/11/02. doi: 10.1128/iai.00768-16. PubMed PMID: 27799333; PubMed Central PMCID: PMCPMC5203655.

204. Taganov KD, Boldin MP, Chang KJ, Baltimore D. NF-kappaB-dependent induction of microRNA miR-146, an inhibitor targeted to signaling proteins of innate immune responses. Proceedings of the National Academy of Sciences of the United States of America. 2006;103(33):12481-6. Epub 2006/08/04. doi: 10.1073/pnas.0605298103. PubMed PMID: $16885212 ;$ PubMed Central PMCID: PMCPMC1567904.

205. Bazzoni F, Rossato M, Fabbri M, Gaudiosi D, Mirolo M, Mori L, et al. Induction and regulatory function of miR-9 in human monocytes and neutrophils exposed to proinflammatory signals. Proceedings of the National Academy of Sciences of the United States of America. 2009;106(13):5282-7. Epub 2009/03/18. doi: 10.1073/pnas.0810909106. PubMed PMID: 19289835; PubMed Central PMCID: PMCPMC2664036.

206. Alsaleh G, Suffert G, Semaan N, Juncker T, Frenzel L, Gottenberg JE, et al. Bruton's tyrosine kinase is involved in miR-346-related regulation of IL-18 release by lipopolysaccharideactivated rheumatoid fibroblast-like synoviocytes. Journal of immunology (Baltimore, Md : 1950). 2009;182(8):5088-97. Epub 2009/04/04. doi: 10.4049/jimmunol.0801613. PubMed PMID: 19342689.

207. Banerjee S, Cui H, Xie N, Tan Z, Yang S, Icyuz M, et al. miR-125a-5p regulates differential activation of macrophages and inflammation. The Journal of biological chemistry.

2013;288(49):35428-36. Epub 2013/10/24. doi: 10.1074/jbc.M112.426866. PubMed PMID: 24151079; PubMed Central PMCID: PMCPMC3853290.

208. Sierra-Filardi E, Puig-Kroger A, Blanco FJ, Nieto C, Bragado R, Palomero MI, et al. Activin A skews macrophage polarization by promoting a proinflammatory phenotype and inhibiting the acquisition of anti-inflammatory macrophage markers. Blood. 2011;117(19):5092-101. Epub 2011/03/11. doi: 10.1182/blood-2010-09-306993. PubMed PMID: 21389328.

209. Wang SY, Tai GX, Zhang PY, Mu DP, Zhang XJ, Liu ZH. Inhibitory effect of activin A on activation of lipopolysaccharide-stimulated mouse macrophage RAW264.7 cells. Cytokine. 2008;42(1):85-91. Epub 2008/03/07. doi: 10.1016/j.cyto.2008.01.010. PubMed PMID: 18321725.

210. Ge J, Wang Y, Feng Y, Liu H, Cui X, Chen F, et al. Direct effects of activin A on the activation of mouse macrophage RAW264.7 cells. Cellular \& molecular immunology. 2009;6(2):129-33. Epub 2009/05/01. doi: 10.1038/cmi.2009.18. PubMed PMID: 19403063; PubMed Central PMCID: PMCPMC4002650.

211. Zhou J, Tai G, Liu H, Ge J, Feng Y, Chen F, et al. Activin A down-regulates the phagocytosis of lipopolysaccharide-activated mouse peritoneal macrophages in vitro and in vivo. Cellular immunology. 2009;255(1-2):69-75. Epub 2008/12/19. doi:

10.1016/j.cellimm.2008.11.001. PubMed PMID: 19091310.

212. Pollard JW. Trophic macrophages in development and disease. Nature reviews Immunology. 2009;9(4):259-70. Epub 2009/03/14. doi: 10.1038/nri2528. PubMed PMID: $19282852 ;$ PubMed Central PMCID: PMCPMC3648866. 
213. Guo C, Buranych A, Sarkar D, Fisher PB, Wang XY. The role of tumor-associated macrophages in tumor vascularization. Vascular cell. 2013;5(1):20. Epub 2013/12/10. doi: 10.1186/2045-824x-5-20. PubMed PMID: 24314323; PubMed Central PMCID: PMCPMC3913793. 214. Yang L, Zhang Y. Tumor-associated macrophages: from basic research to clinical application. Journal of hematology \& oncology. 2017;10(1):58. Epub 2017/03/01. doi: 10.1186/s13045-017-0430-2. PubMed PMID: 28241846; PubMed Central PMCID: PMCPMC5329931.

215. Mahlbacher G, Curtis LT, Lowengrub J, Frieboes HB. Mathematical modeling of tumorassociated macrophage interactions with the cancer microenvironment. Journal for immunotherapy of cancer. 2018;6(1):10. Epub 2018/02/01. doi: 10.1186/s40425-017-0313-7. PubMed PMID: 29382395; PubMed Central PMCID: PMCPMC5791333.

216. Macklin P, McDougall S, Anderson AR, Chaplain MA, Cristini V, Lowengrub J. Multiscale modelling and nonlinear simulation of vascular tumour growth. Journal of mathematical biology. 2009;58(4-5):765-98. Epub 2008/09/11. doi: 10.1007/s00285-008-0216-9. PubMed PMID: 18781303; PubMed Central PMCID: PMCPMC3037282.

217. Wu M, Frieboes HB, McDougall SR, Chaplain MA, Cristini V, Lowengrub J. The effect of interstitial pressure on tumor growth: coupling with the blood and lymphatic vascular systems. Journal of theoretical biology. 2013;320:131-51. Epub 2012/12/12. doi: 10.1016/j.jtbi.2012.11.031. PubMed PMID: 23220211; PubMed Central PMCID: PMCPMC3576147.

218. Lewis C, Murdoch C. Macrophage responses to hypoxia: implications for tumor progression and anti-cancer therapies. The American journal of pathology. 2005;167(3):627-35. Epub 2005/08/30. doi: 10.1016/s0002-9440(10)62038-x. PubMed PMID: 16127144; PubMed Central PMCID: PMCPMC1698733.

219. Leonard F, Curtis LT, Yesantharao P, Tanei T, Alexander JF, Wu M, et al. Enhanced performance of macrophage-encapsulated nanoparticle albumin-bound-paclitaxel in hypoperfused cancer lesions. Nanoscale. 2016;8(25):12544-52. Epub 2016/01/29. doi: 10.1039/c5nr07796f. PubMed PMID: 26818212; PubMed Central PMCID: PMCPMC4919151. 220. Nugent LJ, Jain RK. Extravascular diffusion in normal and neoplastic tissues. Cancer research. 1984;44(1):238-44. Epub 1984/01/01. PubMed PMID: 6197161.

221. McDougall SR, Anderson AR, Chaplain MA. Mathematical modelling of dynamic adaptive tumour-induced angiogenesis: clinical implications and therapeutic targeting strategies. Journal of theoretical biology. 2006;241(3):564-89. Epub 2006/02/21. doi: 10.1016/j.jtbi.2005.12.022. PubMed PMID: 16487543.

222. van de Ven AL, Wu M, Lowengrub J, McDougall SR, Chaplain MA, Cristini V, et al. Integrated intravital microscopy and mathematical modeling to optimize nanotherapeutics delivery to tumors. AIP advances. 2012;2(1):11208. Epub 2012/04/11. doi: 10.1063/1.3699060. PubMed PMID: 22489278; PubMed Central PMCID: PMCPMC3321519.

223. Leonard F, Curtis LT, Ware MJ, Nosrat T, Liu X, Yokoi K, et al. Macrophage Polarization Contributes to the Anti-Tumoral Efficacy of Mesoporous Nanovectors Loaded with AlbuminBound Paclitaxel. Frontiers in immunology. 2017;8:693. Epub 2017/07/04. doi: 10.3389/fimmu.2017.00693. PubMed PMID: 28670313; PubMed Central PMCID: PMCPMC5472662.

224. Cui YL, Li HK, Zhou HY, Zhang T, Li Q. Correlations of tumor-associated macrophage subtypes with liver metastases of colorectal cancer. Asian Pacific journal of cancer prevention : APJCP. 2013;14(2):1003-7. Epub 2013/04/30. PubMed PMID: 23621176.

225. Yuan A, Hsiao YJ, Chen HY, Chen HW, Ho CC, Chen YY, et al. Opposite Effects of M1 and M2 Macrophage Subtypes on Lung Cancer Progression. Sci Rep. 2015;5:14273. Epub 
2015/09/25. doi: 10.1038/srep14273. PubMed PMID: 26399191; PubMed Central PMCID: PMCPMC4585843.

226. Frieboes HB, Curtis LT, Wu M, Kani K, Mallick P. Simulation of the Protein-Shedding Kinetics of a Fully Vascularized Tumor. Cancer informatics. 2015;14:163-75. Epub 2015/12/31. doi: 10.4137/cin.S35374. PubMed PMID: 26715830; PubMed Central PMCID: PMCPMC4687979. 227. Liu Y, Cao X. The origin and function of tumor-associated macrophages. Cellular \& molecular immunology. 2015;12(1):1-4. Epub 2014/09/16. doi: 10.1038/cmi.2014.83. PubMed PMID: 25220733; PubMed Central PMCID: PMCPMC4654376.

228. Quatromoni JG, Eruslanov E. Tumor-associated macrophages: function, phenotype, and link to prognosis in human lung cancer. American journal of translational research.

2012;4(4):376-89. Epub 2012/11/13. PubMed PMID: 23145206; PubMed Central PMCID: PMCPMC3493031.

229. Chanmee T, Ontong P, Konno K, Itano N. Tumor-associated macrophages as major players in the tumor microenvironment. Cancers. 2014;6(3):1670-90. Epub 2014/08/16. doi: 10.3390/cancers6031670. PubMed PMID: 25125485; PubMed Central PMCID: PMCPMC4190561.

230. Genard G, Lucas S, Michiels C. Reprogramming of Tumor-Associated Macrophages with Anticancer Therapies: Radiotherapy versus Chemo- and Immunotherapies. Frontiers in immunology. 2017;8:828. Epub 2017/08/05. doi: 10.3389/fimmu.2017.00828. PubMed PMID: 28769933; PubMed Central PMCID: PMCPMC5509958.

231. Vinogradov S, Warren G, Wei X. Macrophages associated with tumors as potential targets and therapeutic intermediates. Nanomedicine (London, England). 2014;9(5):695-707. Epub 2014/05/16. doi: 10.2217/nnm.14.13. PubMed PMID: 24827844; PubMed Central PMCID: PMCPMC4149280.

232. Leimgruber A, Berger C, Cortez-Retamozo V, Etzrodt M, Newton AP, Waterman P, et al. Behavior of Endogenous Tumor-Associated Macrophages Assessed In Vivo Using a Functionalized Nanoparticle. Neoplasia (New York, NY). 2009;11(5):459-IN4. doi: https://doi.org/10.1593/neo.09356.

233. Singh S, Mehta N, Lilan J, Budhthoki MB, Chao F, Yong L. Initiative action of tumorassociated macrophage during tumor metastasis. Biochimie Open. 2017;4:8-18. doi: https://doi.org/10.1016/i.biopen.2016.11.002.

234. Medrek C, Pontén F, Jirström K, Leandersson K. The presence of tumor associated macrophages in tumor stroma as a prognostic marker for breast cancer patients. BMC Cancer. 2012;12(1):306. doi: 10.1186/1471-2407-12-306.

235. Williams CB, Yeh ES, Soloff AC. Tumor-associated macrophages: unwitting accomplices in breast cancer malignancy. NPJ breast cancer. 2016;2. Epub 2016/03/22. doi:

10.1038/npjbcancer.2015.25. PubMed PMID: 26998515; PubMed Central PMCID: PMCPMC4794275.

236. Wang $H$, Shao $Q$, Sun J, Ma C, Gao W, Wang Q, et al. Interactions between colon cancer cells and tumor-infiltrated macrophages depending on cancer cell-derived colony stimulating factor 1. Oncoimmunology. 2016;5(4):e1122157. Epub 2016/05/04. doi: 10.1080/2162402x.2015.1122157. PubMed PMID: 27141406; PubMed Central PMCID: PMCPMC4839327.

237. Su MJ, Aldawsari H, Amiji M. Pancreatic Cancer Cell Exosome-Mediated Macrophage Reprogramming and the Role of MicroRNAs 155 and 125b2 Transfection using Nanoparticle Delivery Systems. Sci Rep. 2016;6:30110. Epub 2016/07/23. doi: 10.1038/srep30110. PubMed PMID: 27443190; PubMed Central PMCID: PMCPMC4957091. 
238. Correa LH, Correa R, Farinasso CM, de Sant'Ana Dourado LP, Magalhaes KG. Adipocytes and Macrophages Interplay in the Orchestration of Tumor Microenvironment: New Implications in Cancer Progression. Frontiers in immunology. 2017;8:1129. Epub 2017/10/04. doi:

10.3389/fimmu.2017.01129. PubMed PMID: 28970834; PubMed Central PMCID: PMCPMC5609576.

239. Daster S, Amatruda N, Calabrese D, Ivanek R, Turrini E, Droeser RA, et al. Induction of hypoxia and necrosis in multicellular tumor spheroids is associated with resistance to chemotherapy treatment. Oncotarget. 2017;8(1):1725-36. Epub 2016/12/15. doi: 10.18632/oncotarget.13857. PubMed PMID: 27965457; PubMed Central PMCID: PMCPMC5352092.

240. Bredholt G, Mannelqvist M, Stefansson IM, Birkeland E, Bo TH, Oyan AM, et al. Tumor necrosis is an important hallmark of aggressive endometrial cancer and associates with hypoxia, angiogenesis and inflammation responses. Oncotarget. 2015;6(37):39676-91. Epub 2015/10/21. doi: 10.18632/oncotarget.5344. PubMed PMID: 26485755; PubMed Central PMCID: PMCPMC4741854.

241. Lam JS, Shvarts O, Said JW, Pantuck AJ, Seligson DB, Aldridge ME, et al. Clinicopathologic and molecular correlations of necrosis in the primary tumor of patients with renal cell carcinoma. 2005;103(12):2517-25. doi: doi:10.1002/cncr.21127.

242. Lee SY, Ju MK, Jeon HM, Jeong EK, Lee YJ, Kim CH, et al. Regulation of Tumor Progression by Programmed Necrosis \%J Oxidative Medicine and Cellular Longevity. 2018;2018:28. doi: $10.1155 / 2018 / 3537471$.

243. Zanoni M, Piccinini F, Arienti C, Zamagni A, Santi S, Polico R, et al. 3D tumor spheroid models for in vitro therapeutic screening: a systematic approach to enhance the biological relevance of data obtained. Scientific Reports. 2016;6:19103. doi: 10.1038/srep19103

https://www.nature.com/articles/srep19103\#supplementary-information. 244. Lautt WW. Hepatic vasculature: a conceptual review. Gastroenterology. 1977;73(5):1163-9. Epub 1977/11/01. PubMed PMID: 332580.

245. Weagel E, Smith, C., Liu, P.G., Robison, R., O'Neill, K. Macrophage polarization and its role in cancer. J Clin Cell Immunol. 2015;6:338. doi: 10.4172/2155-9899.1000338.

246. Iredale JP, Thompson A, Henderson NC. Extracellular matrix degradation in liver fibrosis: Biochemistry and regulation. Biochimica et biophysica acta. 2013;1832(7):876-83. Epub 2012/11/15. doi: 10.1016/j.bbadis.2012.11.002. PubMed PMID: 23149387. 


\begin{tabular}{|l|l|}
\hline ALD & Alcoholic liver disease \\
\hline Ang2 & Angiopoietin 2 \\
\hline ANOVA & Analysis of variance \\
\hline AUD & Alcohol use disorder \\
\hline BMDM & Bone marrow derived macrophage \\
\hline ECM & Extracellular matrix \\
\hline ELISA & Enzyme-linked immunosorbent assay \\
\hline EtOH & Ethanol \\
\hline HCC & Hepatocellular carcinoma \\
\hline LECM & Lyophilized extracellular matrix \\
\hline LPS & Lipopolysaccharide \\
\hline MMP & Matrix metalloprotease \\
\hline NIAAA & National Institute on Alcohol Abuse and Alcoholism \\
\hline PBS & Phosphate-buffered saline \\
\hline PFA & Paraformaldehyde \\
\hline qRT-PCR & Quantitative reverse transcription polymerase chain reaction \\
\hline RGD & arginine-glycine-aspartic acid domain \\
\hline SEM & Standard error of the mean \\
\hline TACE & TNFa-converting enzyme \\
\hline TAM & Tumor-associated macrophages \\
\hline
\end{tabular}




\begin{tabular}{|l|l|}
\hline TME & Tumor microenvironment \\
\hline TNFa & Tumor necrosis factor-alpha \\
\hline VEGF & Vascular endothelial growth factor \\
\hline
\end{tabular}




\title{
CURRICULUM VITAE
}

\author{
Shanice V. Hudson \\ University of Louisville School of Interdisciplinary and Graduate Studies \\ Department of Pharmacology and Toxicology \\ Louisville, KY 40292 \\ shanice.hudson@louisville.edu \\ 502-852-5157
}

\section{EDUCATION}

1996-2000; 2013 B.S. in Biology, Massachusetts Institute of Technology, Cambridge, MA

\section{PROFESSIONAL EXPERIENCE}

2013-present Graduate Research Assistant, University of Louisville, Department of Pharmacology and Toxicology

2004-2013 Senior Technical Services Representative, Harlan Laboratories (now Envigo)

\section{PROFESSIONAL MEMBERSHIPS AND LEADERSHIP ACTIVITIES}

2015-present Society of Toxicology (SOT), Student Member

2017 Chair, Inflammation: Methods and Mechanisms Poster Section, SOT Annual Meeting

2016-2017 Statistician, University of Louisville Climate Committee, Office of Community Engagement and Diversity

2016-2017 SOT Biological Modeling Specialty Section

2016-2017 Ohio Valley Society of Toxicology (OVSOT)

2015-present American Association for the Study of Liver Diseases (AASLD)

2012-2015 American Association for Cancer Research (AACR)

2014-2015 American Society of Clinical Oncology (ASCO) 
2014-2015 Vice President, Black Biomedical Graduate Student Organization, University of Louisville

2014-2015 Parliamentarian, Minority Association of Graduate Students, University of Louisville

2013-2014 World Molecular Imaging Congress (WMIC)

2011-2015 Minorities in Cancer Research (MICR/AACR); Women in Cancer Research, (WICR/AACR)

2012 American Association of Laboratory Animal Science (AALAS), District 5 Meeting, Program Planning Committee

\section{HONORS AND AWARDS}

2017 Graduate Research Fund Award, University of Louisville

2017 School of Interdisciplinary and Graduate Studies Travel Award

2015 School of Interdisciplinary and Graduate Studies Travel Award

2014 School of Interdisciplinary and Graduate Studies Travel Award

2013 School of Interdisciplinary and Graduate Studies Travel Award

\section{PROFESSIONAL ENGAGEMENT}

2013-present External Reviewer for Peer-Reviewed Journals: Nature Scientific Reports; JAALAS

2017 NIH/DHHS/NIDA Diversity Supplement Workshop (NIH, Bethesda, MD)

2018 National Faculty Women of Color in the Academy Annual Conference (Virginia Tech, VA)

2015 Judge, Research!Louisville NIH R25 Cancer Education Program Poster Competition, Louisville, KY

\section{FUNDING}

2015-2018 NIH Diversity Supplement Award (Grant Number: 3 R01 AA02197802S1)

2018 Doctoral Dissertation Completion Award, University of Louisville

2013-2015 School of Interdisciplinary and Graduate Studies Sponsored Research Award 


\section{ABSTRACTS AND PRESENTATIONS}

1. Hudson, S.V., Dolin, C.E., Merchant, M.L., Arteel, G.E. (2017). Arsenic attenuates LPS-stimulated polarization in murine bone marrow derived macrophages: Potential role of miR-301b. The Toxicologist. Supplement to Toxicological Sciences 151(1):2992.

2. Hudson S.V., Dolin C.E., Merchant M.L., Arteel G.E. (2017) Role of miR-301b in arsenic-altered macrophage polarization: Potential insight into impact of arsenic exposure on innate immune response. Hepatology 66(S1).

3. Poole L.G., Beier J.I., Hudson S.V., Young J.L., Roman J., Marie J.M., Arteel G.E. (2017) A novel nanoparticle-based approach to study axes between the liver and other organs. Hepatology 66(S1).

4. Hudson, S.V., Dolin, C.E., Poole, L.G., Massey, V.L., Wilkey, D., Merchant, M.L., Frieboes, H.B., Arteel, G.E. (2016). Modeling of the kinetics of integrin receptor binding to extracellular matrix proteins. The Toxicologist. Supplement to Toxicological Sciences 150(1):285.

5. Lang, A.L., Kaelin, B.R., Yeo, H., Hudson, S.V., McKenzie, C.M., Sharp, C.N., Poole, L.G., Arteel, G.E., and Beier, J.I. (2016). Critical role of mammalian target of rapamycin (mTOR) in liver damage caused by VC metabolites in mice. The Toxicologist. Supplement to Toxicological Sciences 150(1):231.

6. Hudson, S.V., Kimbrough, C.W., Egger, M., Khanal, A., Smith, M.E., Grizzle, W.E., McNally, L.R. (2014). Detection and characterization of regions of hypoxia within orthotopic pancreatic tumors using multispectral optoacoustic tomography [abstract]. In: Proceedings of the $105^{\text {th }}$ Annual Meeting of the American Association for Cancer Research; 2014 Apr 5-9; San Diego, Ca: AACR; 2014. Abstract nr 4301.

7. Huang, J., Kimbrough, C.W., Hudson, S.V., Yin, W., Rush, J., Ceresa, B.P., Frieboes, H.B., McNally, L.R. (2014). Identification of orthotopic pancreatic adenocarcinoma using multispectral optoacoustic tomography [abstract]. In: Proceedings of the $105^{\text {th }}$ Annual Meeting of the American Association for Cancer Research; 2014 Apr 5-9; San Diego, Ca: AACR; 2014. Abstract nr 2049.

8. Kimbrough, C., Hudson, S., Khanal, A., and McNally L. (2014). Detection of Syndecan-1 probe localization to pancreatic adenocarcinoma using multispectral optoacoustic tomography. J Nucl Med, 55(Supplement 1):337.

9. Hudson, S.V., Huang, J., England, C., Frieboes, H.B., McNally, L.R. (2013). Detection of hypoxia in orthotopic pancreatic tumors in vivo using multispectral optoacoustic tomography [abstract]. In: Proceedings of the $6^{\text {th }}$ Annual Meeting of the World Molecular Imaging Congress; 2013 Sep 18-21; Savannah, GA: WMIC; 2013. p 71. Abstract nr P252.

10. England, C., Hudson, S.V., Huang, J., Yin, W., Frieboes, H., McNally, L.R. (2013). Detection of orthotopic pancreatic tumors using multispectral optoacoustic tomography [abstract]. In: Proceedings of the $6^{\text {th }}$ Annual Meeting of the World Molecular Imaging Congress; 2013 Sep 18-21; Savannah, GA: WMIC; 2013. p 87. Abstract nr LBAP114.

11. England, C., Hudson, S.V., Huang, J., Yin, W., Frieboes, H., McNally, L.R. (2013). $\mathrm{X}$-ray skeleton imaging in conjunction with bioluminescence imaging does not alter 
pancreatic tumors [abstract]. In: Proceedings of the $6^{\text {th }}$ Annual Meeting of the World Molecular Imaging Congress; 2013 Sep 18-21; Savannah, GA: WMIC; 2013. p 87. Abstract nr LBAP119.

12. Williams, S.V., Wildt, S.J., Brooks, A.I., Franklin, C., Livingston, B., Ericsson, A., Wiedmeyer, C.E., Neubauer, B.L., Clutinger, C., Flack, S. and Cooper, D.M. Characterization of the hairless NOD.SCID mouse model (SHrN ${ }^{\mathrm{TM}}$ ) [abstract]. In: Proceedings of the $64^{\text {th }}$ American Association for Laboratory Animal Science Meeting; 2010 Oct 10-14; Atlanta, GA: AALAS; JAALAS; 49(5):655-749: Abstract nr P177.

13. Williams, S.V., Wildt, S.J., Brooks, A.I., Franklin, C., Livingston, B., Cooper, D.M. The SHrN ${ }^{\mathrm{TM}}$ scid hairless NOD mouse model: Development and characterization [abstract]. In: Proceedings of the $101^{\text {st }}$ Annual Meeting of the American Association for Cancer Research; 2010 Apr 17-21; Washington, DC. Philadelphia (PA): AACR; Cancer Res 2010; 70(8 Suppl): Abstract nr 393.

14. Horn, M.J., Williams, S.V., Bostrom, L.A., Cooper, D.M. The impacts of cage density, sanitation frequency, and bedding type on selected measures of animal wellbeing, health, and cage environment [abstract]. In: Proceedings of the $64^{\text {th }}$ American Association for Laboratory Animal Science Meeting; 2010 Oct 10-14; Atlanta, GA: AALAS; JAALAS; 49(5):655-749: Abstract nr PS51.

15. Williams, S.V., Johnson, D.K., Bostrom, L.A., Fredenburg, N.J., Cooper, D.M. Evaluation of housing and sanitation practices as compared to Guide recommendations using laboratory animal performance indices [abstract]. In: Proceedings of the $63^{\text {rd }}$ American Association for Laboratory Animal Science Meeting; 2009 Nov 8-12; Denver, CO: AALAS; JAALAS; 48(5):532-640: Abstract nr P149.

\section{PUBLICATIONS}

1. Hudson, S.V., Dolin, C.E., Poole, L.G., Massey, V.L., Wilkey, D., Beier, J.I., Merchant, M.L., Frieboes, H.B., Arteel, G.E. (2017). Modeling the kinetics of integrin receptor binding to hepatic extracellular matrix proteins. Scientific Reports, 7:12444.

2. Massey, V., Dolin, C.E., Poole, L., Hudson, S.V., Siow, D., Brock, G., Merchant, M., Wilkey, D., Arteel, G. (2017). The hepatic "matrisome" responds dynamically to injury: characterization of transitional changes to the extracellular matrix. Hepatology, 65(3):969-82. DOI: 10.1002/hep.28918.

3. Hudson, S.V., Huang, J.S., Yin, W., Albeituni, S., Rush, J., Khanal A., Yan, J., Ceresa, B.P., Frieboes, H.B., and McNally, L.R. (2014). Targeted noninvasive imaging of EGFR-expressing orthotopic pancreatic cancer using multispectral optoacoustic tomography. Cancer Research, 74; 6271-9.

4. Kimbrough, C.W., Hudson, S., Khanal, A., Egger, M.E., and McNally L.R. (2015). Orthotopic pancreatic tumors detected by optoacoustic tomography using Syndecan1. J Surg Res, 193(1):246-54. 
5. Horn, M.J., Hudson, S.V., Bostrom, L.A. and Cooper, D.M. (2012). Effects of cage density, sanitation frequency, and bedding type on animal wellbeing and heath and cage environment in mice and rats. Journal of the American Association for Laboratory Animal Science, 51(6):781-8. 